DEPARTAMENTO DE INGENIERÍa TELEMÁTICA Y ElECTRÓNICA

EsCuela TÉCNICA SUPERIOR DE INGENIERÍA Y SistemaS DE

TELECOMUNICACIÓN

\title{
Energy Consumption Reduction on High Performance Embedded Systems for Hyperspectral Imaging Cancer Detection
}

\author{
TESIS DOCTORAL
}

\section{Autor: Daniel Madroñal Quintín}

Graduado en Ingeniería Electrónica de Comunicaciones por la Universidad Politécnica de Madrid Máster Universitario en Ingeniería de Sistemas y Servicios para la Sociedad de la Información por la Universidad Politécnica de Madrid

\section{Director: Eduardo Juárez Martínez}

Docteur Ès Sciences Techniques por l'École Polytechnique Fédéral de Lausanne

Director: César Sanz Álvaro

Doctor Ingeniero de Telecomunicación por la Universidad Politécnica de Madrid 



\section{Tribunal}

Tribunal nombrado por el Mgfco. y Excmo. Sr. Rector de la Universidad Politécnica de Madrid, el día de de

\section{Presidente: - \\ Vocales: \\ Secretario: \\ Suplentes:}

Realizado el acto de lectura y defensa de la Tesis el día de de en la Escuela Técnica Superior de Ingeniería y Sistemas de Telecomunicación de la Universidad Politécnica de Madrid.

Calificación:

El Presidente

LOS VOCALES

El Secretario 

A mis padres 



\section{Acknowledgments}

En primer lugar, quería dar las gracias a mis directores de tesis, Edu y César, por haberme abierto las puertas del laboratorio hace ya 7 años. Gracias a vosotros he podido descubrir que quiero dedicar mi vida a la investigación, y que es un mundo lleno de posibilidades del que es imposible cansarse.

También quiero agradecer a la gente con la que trabajé codo con codo en HELICoiD. Himar, Samu, Gustavo, vosotros fuisteis los primeros investigadores con los que tuve el placer de trabajar. Mientras disfrutaba colaborando en un tema tan maravilloso como HELICoiD, pasasteis, casi sin darme cuenta, de compañeros de trabajo a amigos. Con vosotros he aprendido que, con pasión y dedicación, se puede lograr lo que parece imposible a primera vista.

I would also like to thank all the people I have worked with during CERBERO project. The collaborations with INSA, UniCA, UniSS and CEI-UPM largely enriched both my PhD and myself. I learned so many things while working with you, but the one I am proud the most is being able to discuss almost any topic. You opened my mind and taught me to think out of the box, which will be extremely useful in my future.

During this PhD, I had the great opportunity of doing my research stay at Kalray. In particular, I would like to thank Benoît for making this collaboration possible. I am also thankful for the support received by all the people working at Kalray, especially the one from Julien and Stéphane. But the time I spent in Grenoble would not have been the same without the friends I made during this stay. Thomas, Mykhailo, Mattheus, César, Cyril, Arthur, thanks for all those great moments.

Además, este tiempo siempre he estado rodeado de un gran grupo de investigación, el GDEM. De los profesores, Fer, Matías, Pedro y Ángel, me llevo la cercanía y el buen rollo que siempre se ha respirado en el laboratorio, siempre es un gusto trabajar así. También tengo mucho que agradecer a Miguel y a Rubén, con vosotros empezó todo y siempre habéis estado ahí en momentos de crisis y, ahora, ya veis, el niño-melón por fin acaba la tesis. Tampoco me puedo olvidar de mis compañeros de llorería, Jaime, Gema, Anup, Luisa, Marta, os quedáis al cargo del fuerte, hacedme sentir orgulloso.

Por otro lado, siempre le estaré agradecido a toda esa gente que me ha ayudado a despejarme cuando la tesis podía conmigo. No podía haber pedido estar rodeado de gente mejor, de verdad. Curry, aunque nos veamos un par de veces al año, sé que siempre puedo contar contigo y que esto no va a cambiar por mucho tiempo que pase. Laura, todos esos ratos de bromas continuadas y risas interminables son una de las mejores cosas que puede pedir alguien como yo, gracias por estar ahí. Por último, están estas dos (y ahora tres) personas que siempre he considerado mi familia, y que ellos hicieron oficial hace algo más de un año. Me refiero, por supuesto, a Agustín y Noe, gracias por hacer de ese sentimiento algo mutuo. 
En este tiempo, ha habido dos personas que, poco a poco, han traspasado la barrera de compañeros de trabajo y se han convertido en personas realmente importantes en mi vida. Alf, Titti, vosotros me habéis entendido mejor que nadie tanto en los buenos como en los malos momentos. Jamás podré contar las veces que, gracias al consejo de sabios, no he tirado la toalla y he conseguido sobreponerme a cada situación. Gracias infinitas por el apoyo imprescindible que habéis supuesto para mí.

Quería tener un agradecimiento especial para una persona excepcional a la que tengo el placer de considerar mi mejor amiga, Mery. Eres y siempre serás un pilar fundamental en mi vida. Me has demostrado en incontables ocasiones que puedo contar contigo tanto para lo bueno como para lo malo. Da igual cuántos años pasen, sé que siempre nos tendremos el uno al otro cuando nos necesitemos, sobre todo si hay pizza de por medio, ¿verdad?

No podré nunca dejar de agradecer también a mi familia, a todos. A mis padres, por haber estado siempre a mi lado sin importar ni la distancia, ni el humor de ese día... Ni siquiera un confinamiento ha podido con nosotros. A mi hermano, por creer siempre en mí y en que era capaz de cualquier cosa. A Loly, Segu, Alfredo, Belén y Lidia, por estar siempre conmigo sin importar la distancia que nos separe.

Por último, esto no podría acabar de otra manera más que mostrando el mayor de mis agradecimientos hacia ti, Raquel. Tú lo has sido todo durante este tiempo. Has sido mi compañera de trabajo, y contigo he alcanzado todos y cada uno de los logros que se han puesto a nuestro alcance. También has sido una amiga, ayudándome a superar todos los baches que se interponían en mi camino, sin dudar ni un solo instante de que yo era capaz de superar cualquier cosa. Pero, por encima de todo, siempre has sido tú, esa persona con la que siempre soñé compartir mi vida. Tú has sido la que ha hecho posible que yo sea como soy ahora, y que haya conseguido todo lo que me he propuesto. Sé que, si estamos juntos, nada ni nadie podrá pararnos nunca. Eres una persona increíble, y estoy tremendamente orgulloso de ti. Gracias por todo. Te quiero. 


\section{Resumen}

Con el paso del tiempo, la complejidad de las aplicaciones está viéndose incrementada constantemente y, en paralelo, existe un continuo desarrollo de las plataformas de computación sobre las que dichas aplicaciones se ejecutan. Además, los requisitos de los sistemas, tanto funcionales como no funcionales -tiempo de ejecución o consumo de energía-, son cada vez más exigentes, lo que está empezando a convertir el desarrollo de aplicaciones en una tarea inabarcable. Este es el caso del uso de imágenes hiperespectrales - del inglés Hyperspectral Imaging $[\mathrm{HSI}$ - para distinguir el tejido sano del tejido tumoroso en humanos. La investigación en este ámbito ha cobrado importancia en los últimos años debido a que es una tecnología no invasiva y no ionizante capaz de distinguir los márgenes del tumor de manera precisa. Esta aplicación presenta distintos requisitos dependiendo del campo en el que se quiera aplicar: en neurocirugía, es necesario dar una respuesta en tiempo real para ayudar al cirujano durante la operación; en dermatología, sin embargo, el consumo de energía cobra una mayor importancia, ya que, en este caso, sería posible que el sistema estuviera alimentado a través de una batería.

Para facilitar el desarrollo de sistemas en los que existen requisitos tanto funcionales como no funcionales, en la actualidad se utilizan estrategias de diseño que automatizan ciertas tareas, como por ejemplo la paralelización del código, la generación automática del código final, la planificación de tareas, etc. A este respecto, existen unas metodologías de diseño que se basan en el paradigma llamado $Y$ chart, donde se propone una separación entre el desarrollo de la aplicación y la especificación de la plataforma sobre la que se va a ejecutar, para después combinarlos teniendo en cuenta una serie de directrices definidas por los desarrolladores. Además, para llevar a cabo dicha combinación, se pueden utilizar optimizaciones multiobjetivo, donde todos los requisitos del sistema, que pueden incluso ser opuestos entre sí, se intentan cumplir. Teniendo en cuenta esta tendencia, en este documento se propone una metodología de diseño basada en Y-chart y flujo de datos, del inglés dataflow, donde se busca incluir el consumo de energía entre los requisitos no funcionales a optimizar.

Esta nueva metodología consiste en un bucle de optimización del consumo de energía construido con el fin de poderse aplicar independientemente de la aplicación y de la plataforma de computación utilizadas. En concreto, el bucle se ha construido combinando 3 módulos: monitorización del sistema, estimación del consumo de energía y toma de decisiones.

En primer lugar, se ha desarrollado PAPIFY, una infraestructura de monitorización basada en dataflow. Esta herramienta es capaz de obtener información tanto del tiempo de ejecución como de una serie de monitores de rendimiento -del inglés Performance Monitoring Counter (PMC)- durante la ejecución de la aplicación. 
Además, debido a la aproximación dataflow, la información que se quiere monitorizar en cada parte de la aplicación se define de manera individual; asimismo, es posible asociar cada recurso de la plataforma a un conjunto de PMC specífico. Como resultado, con esta herramienta, es posible 1) caracterizar aplicaciones que se ejecuten en plataformas heterogéneas, es decir, que engloben recursos de computación de distinta naturaleza y 2) adaptar la monitorización del sistema automáticamente y de manera transparente al usuario para que, en cada momento, se ajuste a la configuración específica de cada parte de la aplicación, siempre y cuando dicha configuración se soporte en el recurso de computación en el que se esté ejecutando.

Por otro lado, en este documento también se propone una nueva metodología para modelar el consumo de energía de una plataforma de computación. En el desarrollo de esta metodología se ha buscado poder modelar distintos tipos de plataforma, por ejemplo, las denominadas plataformas multi-/many-core, donde un conjunto de procesadores trabajan en paralelo. Para ello, se ha separado el consumo de energía en 3 partes: 1) consumo asociado a la activación de los distintos recursos de la plataforma, 2) consumo debido a las comunicaciones entre recursos de computación y 3) consumo debido al procesamiento dentro de la aplicación. Otra característica importante de la metodología de modelado propuesta es que se desarrollan modelos lineales que utilizan información disponible durante la ejecución, con el objetivo de utilizar los modelos generados para realizar estimaciones del consumo de energía durante la ejecución del sistema.

En el caso de la toma de decisiones para optimizar el consumo de energía del sistema, se propone un mecanismo que construye un bucle de optimización de dicho consumo alrededor de un bloque de mapeado/planificación de tareas que busca optimizar la latencia del sistema. Este bucle consiste en, manteniendo el bloque de mapeado/planificación como una caja negra, caracterizar el rendimiento y el consumo de energía de los sistemas generados y modificar las entradas de dicho bloque en consecuencia. Con estas modificaciones, se prueban distintas configuraciones del sistema con el objetivo de escoger la más eficiente en términos de consumo de energía de entre las que cumplen el requisito de rendimiento del sistema. Esta aproximación se puede utilizar en dos contextos: 1) durante el diseño de la aplicación para explorar el espacio de diseño -del inglés Design Space Exploration (DSE)-, y 2) durante la propia ejecución del sistema.

Para validar la metodología propuesta para optimizar el consumo de energía, durante el desarrollo de esta tesis se han llevado a cabo dos implementaciones: la primera busca mejorar el proceso de DSE en tiempo de diseño, mientras que la segunda, en cambio, está enfocada a la optimización en tiempo de ejecución. En ambos casos, los 3 bloques que se han explicado con anterioridad se combinan para incorporar la optimización del consumo de energía entre las capacidades de las herramientas de diseño basadas en dataflow que se han escogido para implementar el bucle de optimización.

En el caso de la implementación para el tiempo de diseño, se ha escogido un entorno de desarrollo denominado Parallel Real-time Embedded Executives 
Scheduling Method (PREESM). Los sistemas generados usando PREESM se monitorizan automáticamente usando la herramienta PAPIFY para obtener la información asociada al tiempo de ejecución y al consumo de energía de dichos sistemas. Tras esto, los datos recogidos se incluyen en el entorno de desarrollo para optimizar los resultados obtenidos con el proceso de DSE, considerando (o no) el consumo de energía.

De igual modo, el bucle de optimización en tiempo de ejecución se ha embebido en Synchronous Parameterized and Interfaced Dataflow Embedded Runtime (SPiDER), que es el homólogo de PREESM utilizado cuando la aplicación requiere una gestión de recursos dinámica. En este caso, la información se recoge directamente mediante PAPIFY y se incluye dentro de la caracterización interna de la aplicación que tiene SPiDER. Como resultado, SPiDER es consciente en todo momento del estado y el rendimiento de la aplicación que está ejecutando. Con esta extensión se mejora la capacidad de toma de decisiones de SPiDER durante el proceso de mapeado/planificación de tareas, permitiendo así la búsqueda de la configuración de sistema que sea más eficiente en términos de consumo de energía, basándose en información real de la ejecución en curso.

Con el fin de caracterizar los posibles beneficios que se puedan conseguir al aplicar la metodología propuesta, la aplicación que utiliza HSI para detectar tumores se ha implementado a mano en una plataforma many-core llamada Multi-Purpose Processor Array (MPPA). Esta implementación, que se utilizará como base para hacer comparativas, se ha llevado a cabo teniendo como principal objetivo minimizar la latencia de procesamiento, lo que ha llevado a explotar manualmente el paralelismo intrínseco de la aplicación. Al distribuir la carga de trabajo entre los 256 núcleos de procesamiento que tiene la MPPA, se han obtenido aceleraciones de $50 \times$ y $112 \times$ para imágenes de neurocirugía y de dermatología, respectivamente.

Una vez implementada la aplicación a mano, se ha llevado a cabo otra implementación de la aplicación, pero esta vez siguiendo la metodología de diseño Y-chart y el modelado dataflow. En este caso, debido a las limitaciones que conlleva utilizar dicho modelado, los tiempos de procesamiento se han multiplicado por 6 . Sin embargo, una de las ventajas de esta implementación es que se puede utilizar para optimizar automáticamente la latencia y/o el consumo de energía. En el contexto de este documento se han considerado dos escenarios diferentes con los que se pretende caracterizar completamente la metodología de diseño propuesta: 1) optimización de la latencia del sistema y 2) optimización del consumo de energía del sistema fijando un objetivo de rendimiento. En el segundo escenario, además, se han definido una serie de modos de trabajo en los que se ha incrementado/reducido la importancia que tienen tanto el rendimiento de la aplicación como el consumo de energía dentro de los requisitos del sistema.

En el caso de la optimización de latencia, se ha caracterizado la aplicación en términos de tiempos de ejecución utilizando la herramienta PAPIFY, con el fin de proporcionar dicha información tanto a PREESM como a SPiDER. Gracias a esta caracterización, se han conseguido aceleraciones de $1.4 \times$ y $1.3 \times$ al realizar optimizaciones en tiempo de diseño y tiempo de ejecución, respectivamente. 
Por último, queda demostrada la capacidad de reducir el consumo de energía por parte de las dos implementaciones del bucle de optimización propuesto en este documento, ya que, al incluir el consumo de energía como uno de los parámetros no funcionales a optimizar, dicho consumo se ha reducido en hasta un $30.21 \%$ en ambos casos. En este estudio, PAPIFY se ha utilizado para obtener información tanto de los tiempos de ejecución como de los PMC 3 con el fin de hacer estimaciones del consumo de energía utilizando el modelo desarrollado para la MPPA, el cual ha demostrado tener una precisión por encima del 95\% para una aplicación que no se ha utilizado durante el desarrollo del mismo. Como conclusión, se ha demostrado que la metodología propuesta en este documento ha sido capaz de optimizar automáticamente el consumo de energía de un sistema compuesto por una aplicación con una alta demanda de cómputo ejecutada sobre una plataforma compleja. 


\section{Abstract}

The growing complexity of both applications and architectures, combined with the tightening of functional and non-functional system requirements, is reaching the frontiers of what can currently be obtained. This is the case of, for example, the application of Hyperspectral Imaging (HSI) to discern between healthy and tumor human tissues. This research line has gained importance in the past few years, as it is a non-invasive and non-ionizing technology capable of accurately delimiting tumor boundaries. Nevertheless, this kind of system requires processing a large amount of data and, depending on the field in which this application is used, the system requirements vary: in the neurosurgical case, detecting tumor boundaries in realtime is necessary to help the specialist during the surgery; in the dermatological domain, energy consumption becomes the most important requirement, since battery-supplied systems should be used.

To ease the development of systems where functional and non-functional requirements need to be weighed, design strategies where different tasks are automated (parallelization, code generation, task scheduling, etc) are often used. In the literature, the most extended approaches are those based on Y-chart design. This methodology proposes a separation of concerns, where the application is deployed onto the target architecture considering a set of user-defined constraints; additionally, to deploy these systems, multi-objective optimizations can be considered to fulfill the often opposing system constraints. In this regard, this $\mathrm{PhD}$ proposes a Y-chart dataflow-based design methodology whose aim is to include energy consumption within the non-functional requirements to be optimized.

This methodology has been built as an iterative optimization loop with the objective of being both application and architecture independent. Specifically, the proposed energy-aware adaptation loop is composed of 3 different modules: monitoring, energy consumption estimation and energy-aware decision making.

First, concerning the application monitoring, a dataflow-based monitoring infrastructure called PAPIFY has been built. This new tool retrieves timing and Performance Monitoring Counter ( $\mathrm{PMC}$ ) information at runtime, where, due to the dataflow-oriented approach, the instrumentation of each part of the application is individually set up and each platform computational resource is linked to a specific PMC set. As a result, 1) monitoring of heterogeneous architectures is supported and 2) the application profiling is automatically adapted at runtime, considering the part of the application that each processing element is executing.

Secondly, to address the energy consumption estimation, a new platform modeling methodology is proposed focusing on building application-independent models. The approach has been built keeping in mind its potential applicability to model 
architectures of different nature, e.g., multi-/many-cores, which leads to models where the energy consumption is divided into 3 contributions: resource-active, communication and computation. Additionally, linear models based on runtime information are built to be able to estimate the energy consumption at runtime.

Later, regarding the decision making module, an energy-aware mechanism that builds a loop around latency-based mapping/scheduling algorithms to optimize the system energy consumption has been proposed. This loop consists in using the mapping/scheduling block as a black-box, characterizing the performance and energy consumption of its system deployments and modifying its inputs so as to test different system configurations and keep the one with the lowest energy consumption that reaches the desired performance. This approach has been built so as to be applied in both design time, where Design Space Exploration (DSE) is enhanced, and by a runtime resource manager to perform energy-based optimizations.

To validate the methodology, two real implementations of the energy-aware optimization loop have been carried out: one targeting design time DSE enhancement and the other focused on runtime energy consumption optimization. In both cases, the 3 aforementioned modules are combined so as to incorporate energy-awareness capabilities in different dataflow-based design frameworks.

On the one hand, the design time implementation has been included within Parallel Real-time Embedded Executives Scheduling Method (PREESM) design framework, where the generated applications are automatically instrumented using PAPIFY to characterize them in terms of timing and energy consumption. These data is then included within PREESM information so as to enhance its latency-based DSE process considering (or not) energy-awareness.

On the other hand, the runtime version of the energy-aware optimization loop has been embedded within Synchronous Parameterized and Interfaced Dataflow Embedded Runtime SPiDER runtime manager, which is the runtime counterpart of PREESM framework. In this case, the system is iteratively improved with on-thefly information retrieved thanks to PAPIFY and fed to the runtime manager itself. By including this self-awareness of the system execution, SPiDER is able to perform better decisions during the mapping/scheduling process and, on top of that, to seek the most energy-efficient system configuration based on data of its current execution.

To characterize the benefits of applying the proposed methodology, as a baseline to compare with, the HSI cancer detection processing chain has been manually deployed on a many-core architecture called Multi-Purpose Processor Array (MPPA). The main objective of this implementation has been to minimize the latency by exploiting the intrinsic parallelism of the application. As a result, speedups from $50 \times$ to $112 \times$ have been achieved when distributing the workload among the 256 Processing Elements (PE)) of the MPPA using neurosurgical and dermatological images, respectively.

After that, the Y-chart design methodology has been used to define a dataflow version of the application. In this case, in average, the maximum reachable performance has been reduced by 6 due to the limitations of this approach. 
Nevertheless, the systems generated with this implementation can be automatically optimized in terms of latency or energy consumption. Consequently, two scenarios have been considered to fully characterize the proposed methodology: 1) latencybased optimization and 2) energy-awareness considering a specific performance objective. In the latter, additionally, different working modes are used, weighing performance and energy consumption constraints.

In the case of latency-based optimization, both PREESM and SPiDER frameworks have been enhanced with timing profiling information retrieved via PAPIFY. Thanks to this information, speedups up to $1.4 \times$ and $1.3 \times$ are achieved at design time and runtime, respectively.

Finally, promising results have been obtained in both energy-aware optimization loop implementations, as they have been able to reduce the system energy consumption in up to a $30.21 \%$ in both design time and runtime. In this case, PAPIFY retrieves both timing and PMC information so as to estimate the energy consumption using the model developed for the MPPA, whose estimations -for a real application that has not been included during the architecture modeling- have an accuracy over 95\%. Consequently, the design methodology proposed in this $\mathrm{PhD}$ has been proven to successfully deal with automatic multi-objective optimization of a highly demanding application when deployed on a complex architecture. 



\section{Contents}

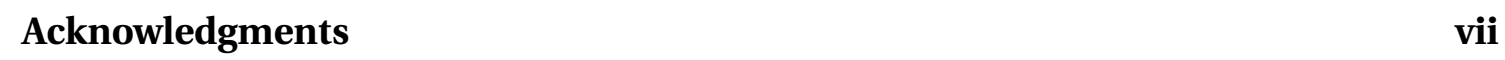

Resumen ix

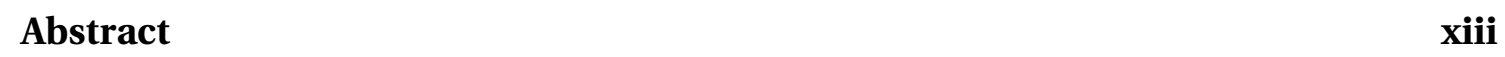

$\begin{array}{ll}\text { Contents } & \text { xvii }\end{array}$

\begin{tabular}{|l|l}
\hline List of Figures & xxi
\end{tabular}

List of Tables $\quad$ xxv

\begin{tabular}{|lc}
\hline Acronyms & xxvii \\
\hline
\end{tabular}

\begin{tabular}{lll}
\hline 1 & Introduction & 1
\end{tabular}

1.1 Motivation . . . . . . . . . . . . . . . . . 1

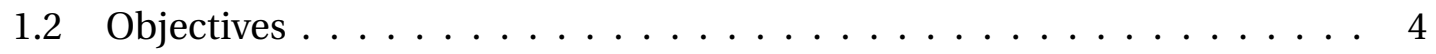

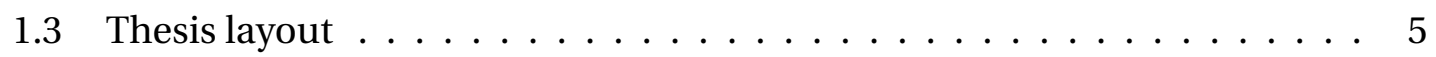

\begin{tabular}{lll}
\hline 2 & State of the art & 7
\end{tabular}

$2.1 \quad$ HSI cancer detection applications $\ldots \ldots \ldots \ldots \ldots$

$2.1 .1 \quad$ Gastrointestinal . . . . . . . . . . . . . . . . 8

$2.1 .2 \quad$ Breast . . . . . . . . . . . . . . . . . . 8

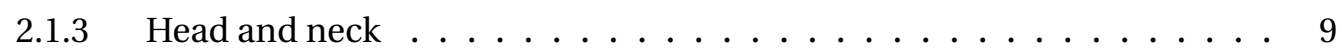

$2.1 .4 \quad$ Skin . . . . . . . . . . . . . . . . . . . 9

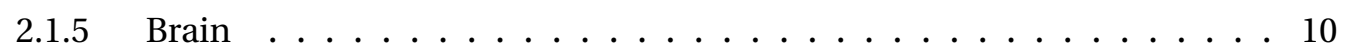

$2.1 .6 \quad$ HELICoiD . . . . . . . . . . . . . . . . . . . . 11

2.2 High performance embedded computing . . . . . . . . . . . . . . . . 12

$2.2 .1 \quad$ GPU platform . . . . . . . . . . . . . . . . . . 13

$2.2 .2 \quad$ Many-core architecture $\ldots \ldots \ldots \ldots$. . . . . . . . . 14

2.3 Application design methodologies . . . . . . . . . . . . . . . . . . 16

$2.3 .1 \quad$ Parallel programming|API . . . . . . . . . . . . . . . . 16

$2.3 .2 \quad$ Computer aided design $\ldots \ldots \ldots \ldots$. . . . . . . . . . 18

$2.3 .3 \quad$ Dataflow based design $\ldots \ldots \ldots \ldots$

2.3 .4 Multi-objective optimization $\ldots \ldots \ldots \ldots$

2.4 Application monitoring . . . . . . . . . . . . . . . . . . . . . 22

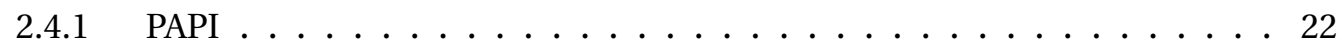

$2.4 .2 \quad$ PMC based monitoring $\ldots \ldots \ldots \ldots$ 
$2.4 .3 \quad$ Dataflow analysis $\ldots \ldots \ldots \ldots \ldots$

2.5 Energy modeling . . . . . . . . . . . . . . . . . . . . 25

2.5 .1 Model definition . . . . . . . . . . . . . . . 26

2.5 .2 Benchmarking . . . . . . . . . . . . . . . . 28

2.5 .3 Model extraction . . . . . . . . . . . . . . . . . 30

2.6 Energy consumption reduction . . . . . . . . . . . . . . 31

$2.6 .1 \quad$ Static consumption reduction . . . . . . . . . . . . . . 31

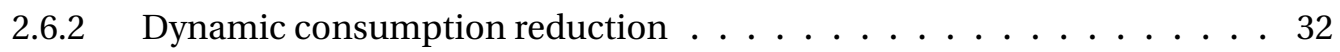

2.7 Discussion . . . . . . . . . . . . . . . . . . . . . . . 34

3 Methodology definition and tools 35

3.1 Use-case . . . . . . . . . . . . . . . . . . . . . . . . . . . . . 35

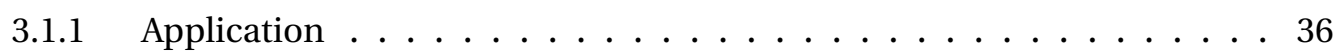

3.1 .2 Architecture . . . . . . . . . . . . . . . . . . . . 39

3.2 Application design methodology specification . . . . . . . . . . . . . . . 40

3.2.1 Application deployment . . . . . . . . . . . . . . . . . 44

3.2 .2 Monitoring . . . . . . . . . . . . . . . . . 41

3.2 .3 KPI estimation . . . . . . . . . . . . . . . . . . 42

3.2 .4 Multi-objective decision making . . . . . . . . . . . . . . 43

3.2 .5 Summary of the methodology $\ldots \ldots \ldots$. . . . . . . . . . 44

3.3 Tools involved . . . . . . . . . . . . . . . . . . . . . . . . 44

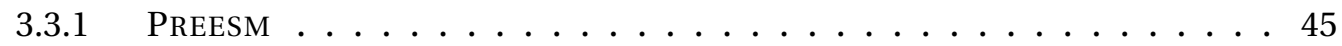

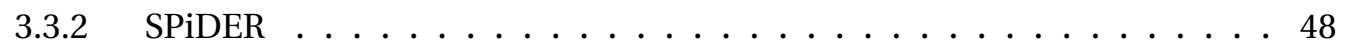

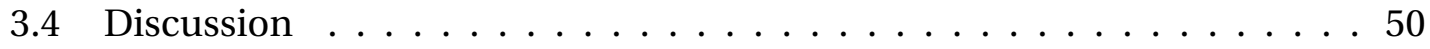

4 Monitoring - PAPIFY

$4.1 \quad$ PAPIFY introduction $\ldots \ldots \ldots \ldots \ldots$. . . . . . . . . . . . . . . 52

4.2 PAPIFY configuration - PAPIFY-DT . . . . . . . . . . . . . . 54

4.3 PAPIFY runtime support - PAPIFY-RT $\ldots \ldots \ldots \ldots$

4.4 PAPIFY-VIEWER . . . . . . . . . . . . . . . . . . . . . . 60

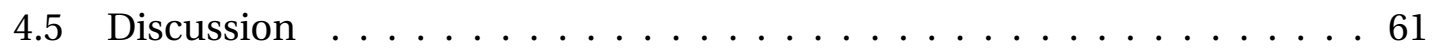

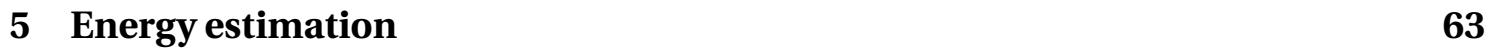

5.1 Modeling methodology overview . . . . . . . . . . . . . . . . 64

5.2 Power measurement procedure . . . . . . . . . . . . . . . 66

5.3 PAPI MPPA support $\ldots \ldots \ldots \ldots \ldots$. . . . . . . . . . . 70

5.4 Resource-active contribution . . . . . . . . . . . . . . 72

5.4 .1 Model definition . . . . . . . . . . . . . . . . . 73

5.4 .2 Benchmark . . . . . . . . . . . . . . . . . . . 74

5.4 .3 Model extraction . . . . . . . . . . . . . . . . . . . . 75 
5.5 Communication contribution $\ldots \ldots \ldots \ldots \ldots$. . . . . . . 77

5.5 .1 Model definition . . . . . . . . . . . . . . . 77

5.5 .2 Benchmark . . . . . . . . . . . . . . . . 80

5.5 .3 Model extraction $\ldots \ldots \ldots \ldots$. . . . . . . . . . 81

5.6 Computation contribution . . . . . . . . . . . . . . . . . . 87

$5.6 .1 \quad$ Model definition $\ldots \ldots \ldots$. . . . . . . . . . . 87

5.6 .2 Benchmark . . . . . . . . . . . . . . . . . . . 89

5.6 .3 Model extraction $\ldots \ldots \ldots \ldots$. . . . . . . . . . . 90

5.7 Platform model . . . . . . . . . . . . . . . . . . . . . . . 99

$5.7 .1 \quad$ MPPA-256-N energy consumption model . . . . . . . . . . . . 99

$5.7 .2 \quad$ Validation $\ldots \ldots \ldots \ldots \ldots$. . . . . . . . . . . . . . . . . . . . . . .

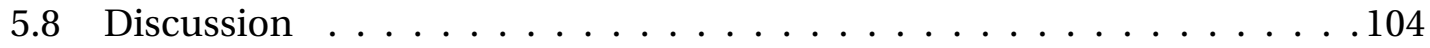

6 Energy-awareness in streaming applications $\quad 105$

6.1 Energy-awareness overview . . . . . . . . . . . . . . . 106

6.2 Energy-aware tuning . . . . . . . . . . . . . . . . . 107

6.3 Design time optimization loop $\ldots \ldots \ldots \ldots$. . . . . . . . . . . . . . .

6.4 Runtime optimization loop . . . . . . . . . . . . . . . . . . . . 116

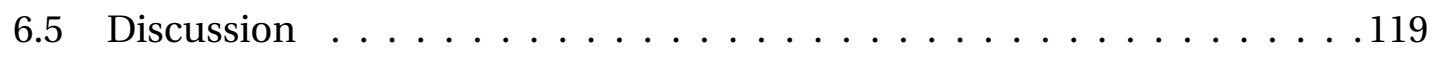

\begin{tabular}{lll}
\hline 7 & Results & 121
\end{tabular}

7.1 Setup . . . . . . . . . . . . . . . . . . . . . . 121

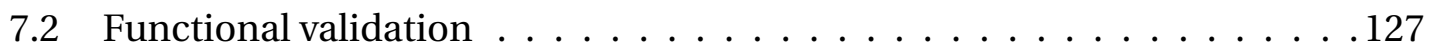

$7.2 .1 \quad$ Implementation by hand . . . . . . . . . . . . . . . . 127

7.2 .2 Results . . . . . . . . . . . . . . . . . . . 137

7.3 Design time optimization $\ldots \ldots \ldots$. . . . . . . . . . . . . . 141

7.3.1 Dataflow-oriented implementation constraints . . . . . . . . . . 142

7.3 .2 Dataflow implementation . . . . . . . . . . . . . . . . . . 144

$7.3 .3 \quad$ Latency-based application optimization $\ldots . . \ldots 150$

$7.3 .4 \quad$ Energy-aware optimization loop . . . . . . . . . . . . . . . . 156

7.4 Runtime optimization . . . . . . . . . . . . . . . . . . . . . . . . . . . 164

7.4 .1 Dataflow implementation with dynamism . . . . . . . . . . . . 164

7.4 .2 Latency-based application optimization . . . . . . . . . . . . . 165

$7.4 .3 \quad$ Energy-aware optimization loop . . . . . . . . . . . . . . . . . 167

$7.4 .4 \quad$ Energy-awareness validation on the MPPA $\ldots \ldots \ldots \ldots 171$

7.5 Discussion . . . . . . . . . . . . . . . . . . . 176

\begin{tabular}{llr}
\hline 8 & Conclusions & 177
\end{tabular}

8.1 Conclusions . . . . . . . . . . . . . . . . . . . . . . . . 177

8.2 Contributions . . . . . . . . . . . . . . . . . . . 179

8.2 .1 HSI cancer detection . . . . . . . . . . . . . . . . . 180 


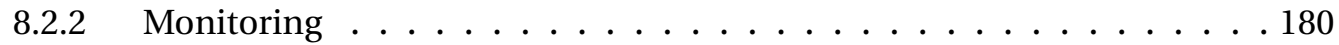

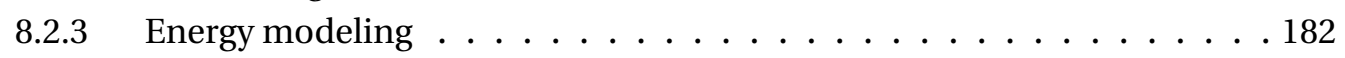

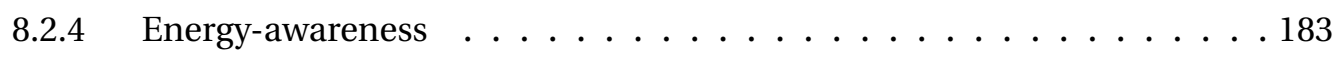

$8.2 .5 \quad$ Iterative energy-aware optimization loop $\ldots \ldots \ldots$. . . . . . . 183

8.3 Impact of the thesis $\ldots \ldots$. . . . . . . . . . . . . . . . 186

$8.3 .1 \quad$ Publications and dissemination . . . . . . . . . . . . . . . 186

8.3 .2 Research projects . . . . . . . . . . . . . . . . . . . 192

$8.3 .3 \quad$ Collaborations $\ldots \ldots \ldots$. . . . . . . . . . . . . . . 193

$8.3 .4 \quad$ Open-access products $\ldots \ldots \ldots 194$

8.3 .5 Grants received . . . . . . . . . . . . . . . . . . . . . 195

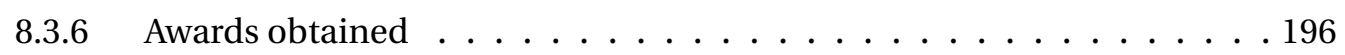

8.4 Future research lines . . . . . . . . . . . . . . . . . . . . . . . 196

$8.4 .1 \quad$ HSI cancer detection application $\ldots . \ldots \ldots 7 . \ldots \ldots$

8.4 .2 Individual blocks extension . . . . . . . . . . . . . . . . 197

$8.4 .3 \quad$ Methodology characterization and extensions $\ldots . \ldots 198$

\begin{tabular}{lr}
\hline Bibliography & 201
\end{tabular} 


\section{List of Figures}

1-1 Y-Chart design flow with feedback at design time and runtime. . . . . . . 3

2-1 $\quad$ HSI technology applied to cancer detection . . . . . . . . . . . . . . 8

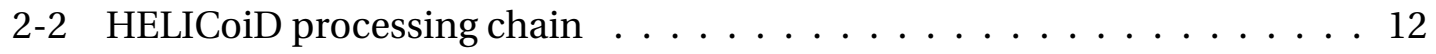

2-3 $\quad$ HELICoiD processing chain partial results . . . . . . . . . . . . . . . . 12

$2-4 \quad$ GPU general structure . . . . . . . . . . . . . . . . . . . . 13

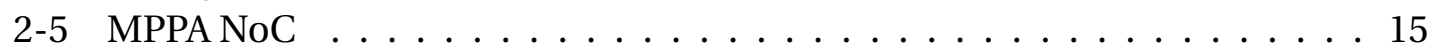

$2-6$ MPPA-256-N Bostan structure . . . . . . . . . . . . . . . . 15

$2-7 \quad$ Basic example of pthreads usage . . . . . . . . . . . . . . . . . 17

2-8 $\quad$ Basic example of OpenMP|fork/join usage . . . . . . . . . . . . . . . . . . . . . . . . . . . 19

2-9 $\quad$ Y-Chart design block diagram . . . . . . . . . . . . . . . . . . . . . 19

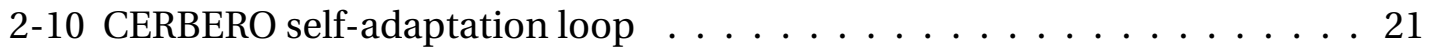

2-11 PAPI library block diagram . . . . . . . . . . . . . . . . . . . . 23

$2-12$ Power consumption schema . . . . . . . . . . . . . . . . . 26

2-13 Levels of abstraction when modeling architectures. . . . . . . . . . . . 27

$2-14$ MoA example . . . . . . . . . . . . . . . . . . . . . . 28

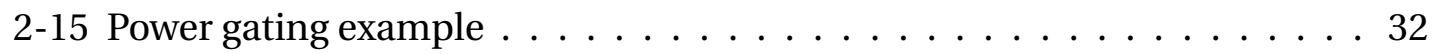

2-16 Clock gating example . . . . . . . . . . . . . . . . . . . . 33

$2-17$ DVFS example . . . . . . . . . . . . . . . . . . . . . 33

2-18 Task migration example . . . . . . . . . . . . . . . . . . . . . 34

3-2 $\quad$ HELICoiD processing chain. Supervised classification step . . . . . . . . . 37

3-4 $\quad$ Iterative Y-chart dataflow-based design methodology $\ldots . . . . . . . . . .440$

3-5 $\quad$ PREESM application graph $\ldots \ldots \ldots \ldots \ldots$. . . . . . . . . . . . . 45

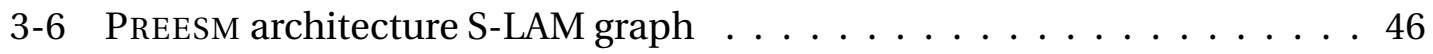

3-7 PrEESM|scenario . . . . . . . . . . . . . . . . . . . 46

3-8 PREESM workflow . . . . . . . . . . . . . . . . . 47

3-9 SRDAG of Sobel-morpho example with a parallelism degree of 4 . . . . . . 48

3-10 SPiDER $\mid$ runtime structure . . . . . . . . . . . . . . . 49

4-1 Iterative Y-chart dataflow-based design methodology-Monitoring . . . . 51

4 4-2 $\quad$ PAPIFY use-case example $\ldots \ldots \ldots \ldots \ldots \ldots$

4 4-3 PAPIFY diagram . . . . . . . . . . . . . . . . . . . . . . 54

4-4 PAPIFY PREESM user interface . . . . . . . . . . . . . . . . . 57

4-5 PAPIFY-SPiDER $\mid$ runtime structure . . . . . . . . . . . . . . . . . . 59

4-6 PAPIFY-VIEWER workload distribution view . . . . . . . . . . . . . . 60

4-7 $\quad$ PAPIFY-VIEWER hardware utilization for PAPI_L1_DCM event view . . . . 60

5-1 Iterative Y-chart dataflow-based design methodology -|KPI|estimation . . 63 
5-2 Representation of the proposed energy consumption model . . . . . . . . 64

5-3 MPPA|block diagram: platform resources . . . . . . . . . . . . . . . 65

5-4 $\quad$ MPPA block diagram:|MPPA transmissions . . . . . . . . . . . . 65

5-5 MPPA|block diagram: Compute Cluster (CC) . . . . . . . . . . . . . . . . . 65

5-6 kl-power measurements for matrix multiplication . . . . . . . . . . . . . 67

5-7 Detail of core k1-power measurements for matrix multiplication . . . . . 67

5-8 $\quad$ Multimeter measurements for matrix multiplication . . . . . . . . . . . . 68

5-9 Detail of multimeter measurements for matrix multiplication . . . . . . . 69

5-11 $\mid$ IO $\mid$ and $\mid \mathrm{CC} /$ resource-active power consumption $\ldots \ldots \ldots$. . . . . . . 75

5-12 Temperature impact on resource-active power consumption . . . . . . . 76

5-15 Energy consumption per transmission - CC-CC communication . . . . . 82

5-16 Energy consumption per transmission - CC-CC communication zoom

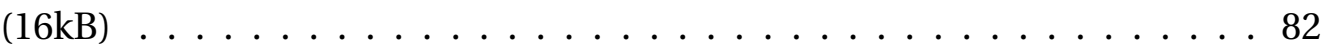

5-17 Energy consumption per transmission - One-sided vs simultaneous . . . 83

5-18 Power contribution comparison:|SMEM|vs|DDR|and read vs write . . . . 84

5-19 Energy consumption per transmission - SMEM|and|DDR $\mid$ usage $\ldots . . .884$

5-20 Energy consumption per transmission - Measurements vs estimation . . 86

5-21 Power contribution comparison - Measurements vs estimation detail

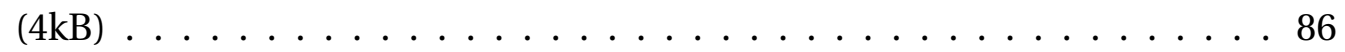

5-23 Energy and events obtained when executing the training set for

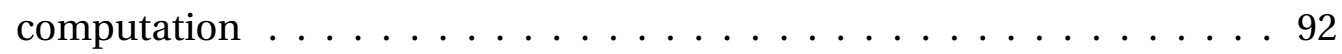

5-24 Computation contribution models analysis - Training samples uniformity 93

5-25 Energy measured vs energy estimated - Computation contribution . . . . 94

5-26 Computation contribution models analysis - Events selection . . . . . . . 95

5-27 Energy consumption for computation - Events occurrences . . . . . . . . 96

5-28 Energy consumption for computation - Energy contribution per event. . 96

5-29 Energy measured vs energy estimated - Computation contribution using only double data types in the training phase . . . . . . . . . . . . 97

5-30 Energy measured vs energy estimated - Computation contribution on

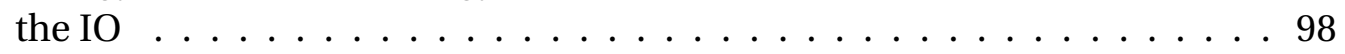

5-31 Model validation -GeMM experiment analysis $\ldots$. . . . . . . . . . . . 103

5-32 Model validation - GeMM experiment energy measurement vs estimationl 03

5-33 Model validation - GeMM experiment energy measurement vs estimation detail using 16|CCs . . . . . . . . . . . . . . . . . . 104

6-1 Iterative Y-chart dataflow-based design methodology-Decision making 105

6-2 $\quad$ Energy-aware optimization loop . . . . . . . . . . . . . . . 107

6-3 $\quad$ SDF Y-Chart flow example . . . . . . . . . . . . . . . . . . . . 108

6-4 $\quad$ Energy-aware tuning algorithm block diagram . . . . . . . . . . . . . . . 109

6-5 Implementation comparative: (a) latency-based vs (b) energy-awareness 110

6-6 $\quad$ Binary search example $\ldots \ldots \ldots \ldots \ldots \ldots \ldots$. . . . . . . . . . . . . . . . . . . . . . .

6-7 $\quad$ Y-Chart dataflow design flow at design time . . . . . . . . . . . . . . . . . 112

6-8 $\quad$ Timings tab in PreEsM $\mid$ scenario . . . . . . . . . . . . . . . . . . . . . 113

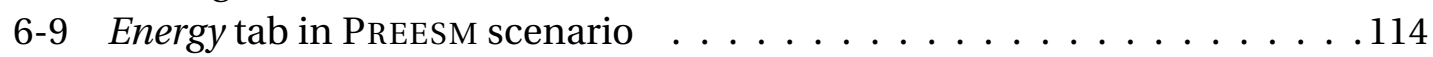


6-10 Energy consumption computation model table in PREESM interface . . . 115 6-11 Y-Chart dataflow design flow at runtime . . . . . . . . . . . . . . . . 117 6-12 SPiDER $\mid$ runtime structure including energy-awareness feedback loop . . 117

7-1 $\quad$ Power and energy reduction equivalence for an infinite time . . . . . . . . 125

7-3 Workload distribution proposed to implement by hand the $/$ HELICoiD processing chain on the MPPA . . . . . . . . . . . . . . . 128

7-4 $\quad$ Band image regions used during $|\mathrm{KNN}|$ searching algorithm $\ldots$. . . . . . . . 134

7-5 Possible execution workflows of the HSI processing chain implementation done by hand on the MPPA $\ldots \ldots \ldots \ldots \ldots \ldots \ldots$. . . . . . . . . . . . . . . .

7-6 Total processing times (s) and speedup (times) of the $\mid$ HSI $\mid$ processing chain. Implementation by hand . . . . . . . . . . . . . . . . . 140

7-7 $\quad$ Third-order tensor representation of hyperspectral images . . . . . . . . . 143

7-8 MPPA communication-processing strategies comparison . . . . . . . . . 144

7-9 HSI processing chain in|PREESM: system-level representation . . . . . . . 145

7-10 HSI processing chain in PREESM| processing chain representation . . . . 146

7-11 HSI processing chain in PREESM:|PCA graph representation $\ldots$. . . . . . . . 147

7-12 HSI processing chain in|PREESM image centering graph representation . 148

7-13 HSI processing chain in|PREESM: $\mid$ SVM top graph representation. . . . . . 148

7-14 HSI processing chain in|PREESM:SSVM|stages graph representation . . . . 149

7-15 HSI processing chain in|PREESM| KNN|graph representation . . . . . . . . 149

7-16 Classification results comparison: implementation done by hand vs dataflow implementation . . . . . . . . . . . . . . . . . . . . . . . . . . .

7-17 Design time latency-based optimization loop results . . . . . . . . . . 156

7-20 MPPA data transmission speed on PREESM . . . . . . . . . . . . . . . . . 159

7-21 PREESM energy-awareness tuning real example . . . . . . . . . . . . . . . . 161

7-22 Design time energy-aware optimization loop results . . . . . . . . . . . . . 163

7-23 SPiDER || HSI processing chain dataflow implementation with dynamic

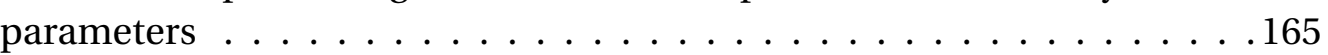

7-24 Runtime latency-based optimization loop results $\ldots$. . . . . . . . . . . 167

7-25 Runtime energy-aware optimization loop results for the x86 architecture 171

\begin{tabular}{l|l|l|l|l|}
\hline 7-26 & MPPA & energy consumption model evaluation running the $\mid$ HSI & cancer
\end{tabular} detection algorithm $\ldots \ldots \ldots \ldots \ldots \ldots \ldots \ldots \ldots$

7-27 Runtime energy-aware optimization loop results for the|MPPA|architec-

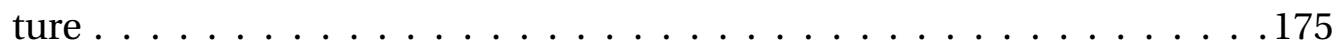





\section{List of Tables}

2-1 Discussion and decisions of each topic presented in the state of the art

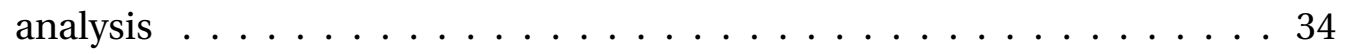

3-1 Application deployment design framework comparative . . . . . . . . . 41

5-1 $\quad$ MPPA block diagrams used to develop the energy consumption model . . 65

$5-2$ Power measurement procedures comparison . . . . . . . . . . . 69

5-3 $\quad$ PMC $\mid$ events available on the $\mid$ MPPA|platform $\ldots \ldots \ldots$. . . . . . . 70

5-4 $\quad$ Preset|PMC|events available on the $\mid$ MPPA|platform $\ldots$. . . . . . . . 71

5-5 $\quad$ Required clock cycles to access the $\mid$ PMC 3 on the $\mid$ MPPA| using different PAPI strategies . . . . . . . . . . . . . . . . 72

$5-6$ Resource-active $\mu$ benchmarks summary $\ldots \ldots \ldots$. . . . . . . . . . 75

5-7 $\quad$ Model evaluation for resource-active . . . . . . . . . . . . . . . . 77

5-8 $\quad$ Hops required on the $\mid$ MPPA|NoC|to communicate among|CC月 $\ldots . . . .88$

5-9 Communication $\mu$ benchmarks summary $\ldots \ldots \ldots \ldots$. . . . . . . . . 81

5-10 Model evaluation for every type of communication . . . . . . . . . . . 86

5-11 Computation $\mu$ benchmarks summary . . . . . . . . . . . . . . 90

5-12 Model evaluation for computation using different training sets . . . . . 97

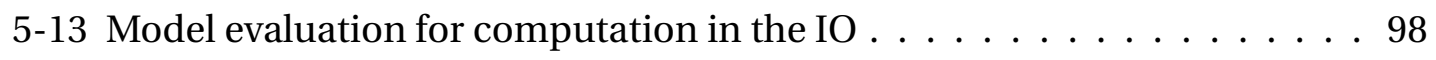

5-14 Workload distribution in $\mid$ GeMM|application with the number of active

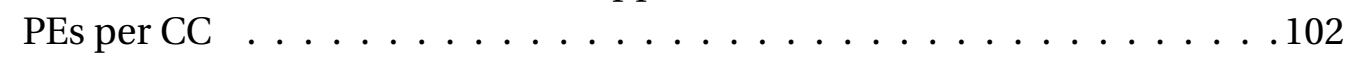

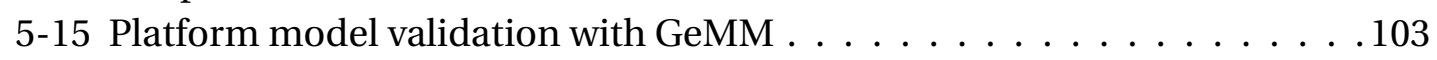

7-1 $\quad$ HSI cancer images used during the validation . . . . . . . . . . . . . . . . 122

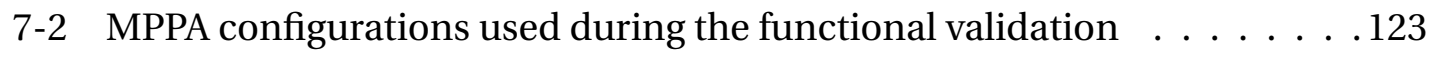

7-3 Working modes used in the Y-chart dataflow-based energy-aware design methodology experiments . . . . . . . . . . . . . . . . . . . . . . . . . . . . . .

7-4 Validation experiment terminology . . . . . . . . . . . . . . . . . . . . . 124

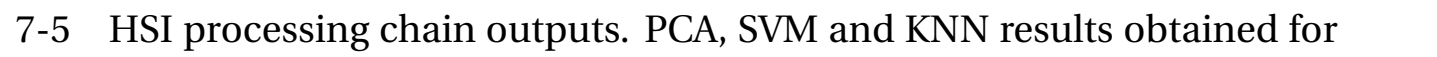
the $\mid$ MPPA implementation using the validation hyperspectral dataset . . 138

7-6 Partial processing times (s) and speedups of the $\mid$ HSI processing chain. Implementation by hand $\ldots \ldots \ldots \ldots \ldots \ldots \ldots$. . . . . . . . . . . . . . . . .

7-7 Performance (images per second) and power (W) of the HSI processing chain. Implementation by hand . . . . . . . . . . . . . . . . 141

7-8 Parameters used in the dataflow implementation of the $\mid$ HSI $\mid$ processing

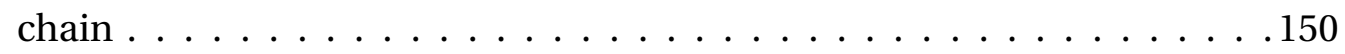

7-9 Dataflow implementation code specialization: parameter values . . . . . 152

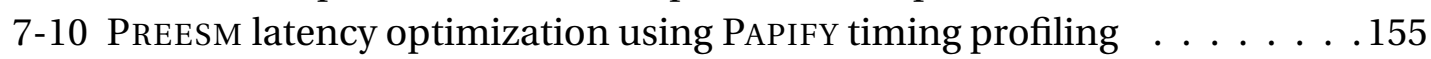


7-11 Performance and power consumption results. Design time energyaware tuning using|PREESM $\ldots \ldots \ldots \ldots \ldots$. . . . . . . . . . . . . . . . .

7-12 Performance, power and power savings of the HSI processing chain. Implementation by hand vs design time energy-aware optimization loop 162

7-13 SPiDER latency optimization using PAPIFY timing feedback . . . . . . . 166

7-14 SPiDER energy-aware optimization experiments order $\ldots$. . . . . . . . . . 168

7-15 Energy-aware performance objectives adapted for the x86 architecture .169

7-16 SPiDER energy-aware system configurations using the energy-aware tuning algorithm and the $\mathrm{x} 86$ 16-core architecture $\ldots \ldots \ldots$. . . . . . 170

7-17 SPiDER energy-aware energy consumption reduction using the energyaware tuning algorithm and the x86 16-core architecture . . . . . . . . .170

7-18 MPPA energy-aware tuning algorithm. Energy consumption measurements results being considered $\ldots \ldots \ldots \ldots \ldots \ldots$. . . . . . . . . . . . . . .

7-19 Performance, power and power savings of the HSI processing chain. Implementation by hand vs runtime energy-aware optimization loop $\ldots 174$ 


\section{Acronyms}

\section{5-ALA 5-Aminolevulinic Acid}

AAM Algorithm Architecture Matching

ALMA Architecture oriented paraLlelization for high performance embedded Multicore systems using scilAb

ALOHA software framework for runtime-Adaptive and secure deep Learning On Heterogeneous Architectures

API Application Programming Interface

Apollo Automatic Speculative Polyhedral Loop Optimizer

ARTICo $^{3}$ Arquitectura Reconfigurable para el Tratamiento Inteligente de Cómputo, Consumo y Confiabilidad

BLAS Basic Linear Algebra Subprograms

CAD Computer Aided Design

CAL Cal Actor Language

CC Compute Cluster

CERBERO Cross-layer modEl-based fRamework for multi-oBjective dEsign of Reconfigurable systems in unceRtain hybRid envirOnments

CMOS Complementary Metal Oxide Semi-conductor

CNN Convolutional Neural Network

CPS Cyber-Physical System

CPU Central Processing Unit

CUDA Compute Unified Device Architecture

DAG Direct Acyclic Graph

DDR Double Data Rate

DL Deep Learning

DMA Direct Memory Access

DNN Deep Neural Network

DPN Dataflow Process Network

DSE Design Space Exploration 


\begin{tabular}{|c|c|}
\hline DSP & Digital Signal Processing \\
\hline DVFS & Dynamic Voltage and Frequency Scaling \\
\hline FAST & Fast Assignment using Search Technique \\
\hline FET & Future and Emerging Technologies \\
\hline FIFO & First-In First-Out queue \\
\hline FPGA & Field Programmable Gate Array \\
\hline FPS & Frames Per Second \\
\hline GDDR & Graphics DDR \\
\hline GeCoS & Generic Compiler Suite \\
\hline GeMM & General Matrix Multiplication \\
\hline GPU & Graphics Processing Unit \\
\hline GRT & Global Run-Time \\
\hline HELICoiD & HypErspectraL Imaging Cancer Detection \\
\hline HLS & High Level Synthesis \\
\hline HPC & High Performance Computing \\
\hline HSI & Hyperspectral Imaging \\
\hline IEEE & Institute of Electrical and Electronics Engineers \\
\hline IETR & Institut d'Électronique et de Télécommunications de Rennes \\
\hline ILP & Integer Linear Programming \\
\hline iMRI & intra-operative Magnetic Resonance Imaging \\
\hline INSA & Institut National des Sciences Appliquées \\
\hline IO & Input/Output Subsystem \\
\hline ISS & Instruction Set Simulator \\
\hline IUMA & Instituto Universitario de Microelectrónica Aplicada \\
\hline iUS & intra-operative Ultrasound \\
\hline KNN & K-Nearest Neighbors \\
\hline KPI & Key Performance Indicator \\
\hline LDA & Linear Discriminant Analysis \\
\hline LPDDR & Low Power DDR \\
\hline LRT & Local Run-Time \\
\hline LSLA & Linear System-Level Architecture \\
\hline
\end{tabular}




\begin{tabular}{|c|c|}
\hline MAPS & MPSoCApplication Programming Studio \\
\hline MDC & Multi-Dataflow Composer \\
\hline MIMD & Multiple Instruction Multiple Data \\
\hline MMSE & Minimum Mean Squared Error \\
\hline MMU & Memory Management Unit \\
\hline MoA & Model of Architecture \\
\hline MoC & Model of Computation \\
\hline MPI & Message Passing Interface \\
\hline MPPA & Multi-Purpose Processor Array \\
\hline MPSoC & Multi-Processor System on Chip \\
\hline MRI & Magnetic Resonance Imaging \\
\hline NIPALS & Non-Linear Iterative Partial Least Squares \\
\hline NoC & Network-on-Chip \\
\hline NUMA & Non-Uniform Memory Access \\
\hline OpenCL & Open Computing Language \\
\hline OpenMP & Open Multi-Processing \\
\hline ORCC & Open RVC,CALCompiler \\
\hline OS & Operating System \\
\hline PAPI & Performance API \\
\hline PaRSEC & Parallel Runtime Scheduling and Execution Controller \\
\hline PaxES & Parallelism Extraction for Embedded Systems \\
\hline PCA & Principal Component Analysis \\
\hline $\mathbf{P E}$ & Processing Element \\
\hline PiSDF & Parameterized and Interfaced Synchronous DataFlow \\
\hline PLATINO & $\begin{array}{l}\text { Plataforma HW/SW distribuida para el procesamiento inteligente de } \\
\text { información sensorial heterogénea en aplicaciones de supervisión de } \\
\text { grandes espacios naturales }\end{array}$ \\
\hline PMC & Performance Monitoring Counter \\
\hline POSIX & Portable Operating System Interface \\
\hline PreesM & Parallel Real-time Embedded Executives Scheduling Method \\
\hline QoS & Quality of Service \\
\hline RAM & Random Access Memory \\
\hline
\end{tabular}




\begin{tabular}{|c|c|}
\hline $\mathbf{R F}$ & Random Forest \\
\hline RGB & Red-Green-Blue \\
\hline $\mathbf{R M}$ & Resource Manager \\
\hline RVC & Reconfigurable Video Coding \\
\hline SAM & Sequential Access Memory \\
\hline SDF & Synchronous DataFlow \\
\hline SIMD & Single Instruction Multiple Data \\
\hline S-LAM & System-Level Architecture Model \\
\hline SM & Streaming Multiprocessor \\
\hline SMEM & Shared Memory \\
\hline SNR(dB) & Signal-to-noise ratio in $\mathrm{dB}$ \\
\hline SoC & System-on-Chip \\
\hline SPiDER & $\begin{array}{l}\text { Synchronous Parameterized and Interfaced Dataflow Embedded } \\
\text { Runtime }\end{array}$ \\
\hline SRDAG & Single-Rate DAG \\
\hline STDEV & Standard Deviation \\
\hline SVM & Support Vector Machine \\
\hline SynDEx & Synchronous Distributed Executive \\
\hline TCT & Tightly-Coupled Thread \\
\hline TLB & Transaction Look-aside Buffer \\
\hline ULPGC & Universidad de Las Palmas de Gran Canaria \\
\hline UniCA & Università degli Studi di Cagliari \\
\hline UniSS & Università degli Studi di Sassari \\
\hline UPM & Universidad Politécnica de Madrid \\
\hline VLIW & Very Long Instruction Word \\
\hline VLSI & Very-Large-Scale Integration \\
\hline WHO & World Health Organization \\
\hline XML & Extensible Markup Language \\
\hline
\end{tabular}




\section{INTRODUCTION}

\subsection{Motivation}

According to World Health Organization WHO 1 , cancer is the second leading cause of death globally, [...] about 1 in 6 deaths in the world is due to cancer. One of the main action points to be improved, highlighted by $\mathrm{WHO}$ itself, is the early diagnosis. When the malignant tumor is detected early, it is more likely for the patient to respond positively to the treatment and, thus, increase their probability of surviving.

However, even if the malignant tumor is detected in an early stage, differentiation of healthy and tumorous tissues is a key aspect to completely remove the malignant tumor and, consequently, drastically reduce cancer redundancy and avoid possible patient's sequels. In this line, there is a technology called Hyperspectral Imaging (HSI) that is gaining momentum among the technologies available to accurately locate tumorous tissues, such as Magnetic Resonance Imaging (MRI) or 5-Aminolevulinic Acid (5-ALA). HSI is a non-contact and non-ionizing technology that has already been used to analyze human cancer of different nature with promising results: breast, gastric, oral, tongue, colon, skin and brain.

This technology collects information of a wide range of wavelengths from across the electromagnetic spectrum, hence, generating both spatial and spectral highresolution images. The objective of this technology is to identify the distribution of materials within a captured scene based on the surface reflectance of each spectral band, the so-called spectral signature. As a result, it is possible to acquire the image directly from the patient (in-vivo procedures) and process it so as to discern between cancerous and healthy tissues in a non-contact way.

Considering the huge amount of data generated by HSI together with the algorithmic complexity required to analyze those images, its processing usually requires lot of time and/or resources. However, there is a growing trend in which not only functional requirements are demanded, but also non-functional ones. These different requirements appear in two state-of-the-art medical HSI applications, such as an application where $\mathrm{HSI}$ is employed to provide information to a neurosurgeon during the operation will only be helpful if the results are provided in real-time. In this context, real-time refers to the time required by the hyperspectral camera to capture a new image, i.e., up to 1 minute. Likewise, using a battery-supplied hand-

[WHOcancer website: https://www.who.int/en/news-room/fact-sheets/detail/cancer 
held system capable of detecting skin cancer in an early stage can be of great help when used in, for example, the family doctor office. However, to be really useful, this system would require both energy efficiency and short processing times. These nonfunctional requirements -real-time and energy efficiency- are also considered the Key Performance Indicators (KPIs) of the system.

In this line, implementing complex algorithms to process hyperspectral images -whose sizes are usually above 100MB- requires, as mentioned before, extensive computational resources. Moreover, providing energy efficiency and/or real-time responses increases even more the performance requirements. As a solution, High Performance Computing (HPC) architectures are the only ones providing enough computation power to fulfill both functional and non-functional specifications.

Consequently, evaluating the best architecture for each application and devising an efficient implementation becomes a really challenging task. Among others, this is due to the peculiarities associated with each platform, e.g., efficient workload distribution, parallelization strategies, bottlenecks, memory accesses, etc. With current state-ofthe-art approaches, achieving well-balanced implementations that fulfill functional and non-functional requirements incurs in ever increasing (experienced) developer times, who struggle to meet all these often opposing criteria. This situation is reaching the frontiers of what can currently be obtained; as an approach to solve this issue, the Y-chart design methodology [Kienhuis'01] tries to bridge this productivity gap by isolating application( and architecture? concerns and merging them under a set of user-defined constraints3.

Combined with the Y-chart design methodology, dataflow Models of Computation (MoCs), Models of Architecture (MoAs, programming methodologies and associated design tools bring the possibility to explore new, improved design flows. This way, Design Space Exploration (DSE) 4 by rapid prototyping of applications on these complex architectures is being tackled through a set of different design automation tools like compiler parallelization techniques, code generation, task scheduling, etc. However, tools based on Y-chart design and dataflow MoCs, such as Open RVC.CAL Compiler (ORCC) |Yviquel'13a|, Parallel Real-time Embedded Executives Scheduling Method (PREESM) [Pelcat'14a| or SCADE [Pagano'18], usually provide a generic solution following a predefined methodology for any application, which could imply that the proposed solution does not fulfill the system specifications.

In order to evaluate the quality of automatic application deployments 5 , it is necessary to analyze the application execution on the target platform. Properly doing so requires having a deep understanding of the specific characteristics of the architecture and its available profiling tools. In this line, information such as memory usage, code parallelization, workload distribution, I/O utilization, etc., can help to discern how the application is behaving when running on a specific platform. To fulfill the so-called monitoring6 task, there is an abstraction layer that exposes a uniform interface to access hardware Performance Monitoring Counters (PMC). Performance API (PAPI) library [Terpstra'10] offers a common architecture-independent layer coupled with an architecture-specific layer to cope with the individual characteristics 
of each architecture. Using PAPI the PMC can be accessed to profile low level events from a processor execution. This information can, in turn, be used to perform KPI estimation 7 from an even higher level of abstraction, e.g., power or energy [Ren'14, Ren'13. The availability of these performance indicators contributes not only to increase designers' productivity, but also enables iterative design flows both taking runtime decisions 8 or refining the user-defined constraints 3 with real information.

For these reasons, this $\mathrm{PhD}$ proposes a new Y-chart design flow, represented in Figure 1-1, including both design time and runtime refinements.

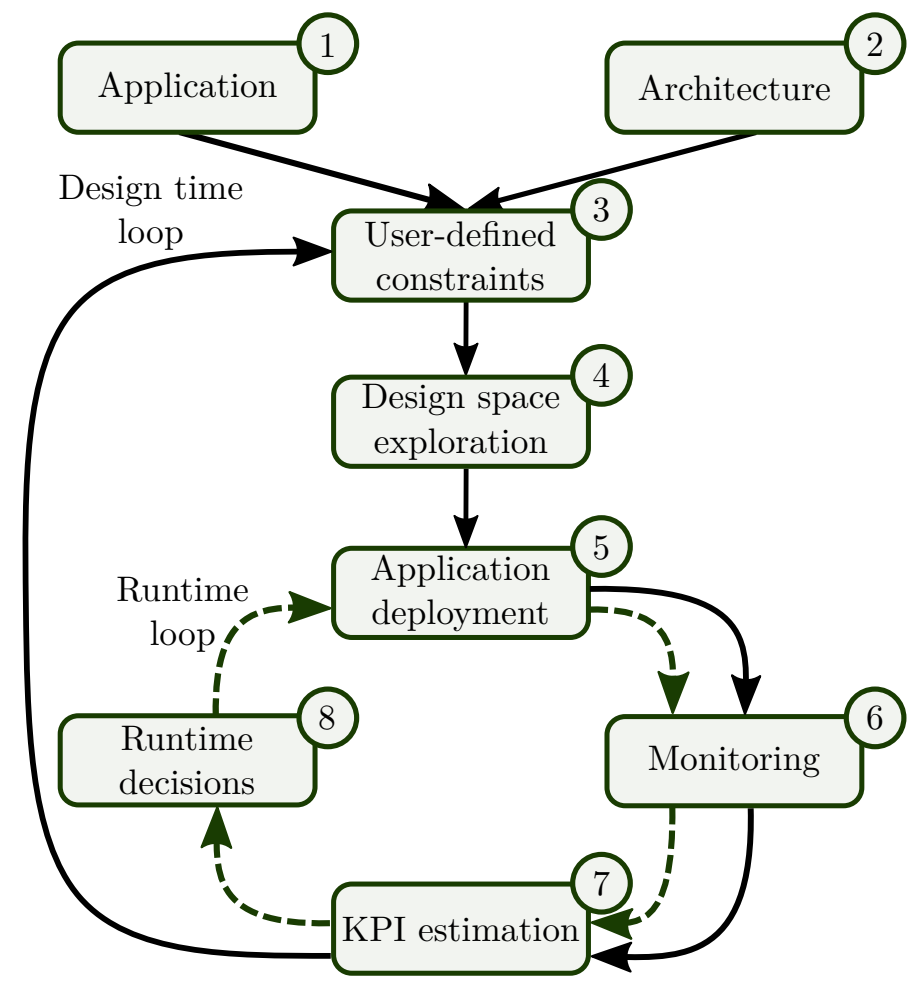

Figure 1-1: Y-Chart design flow with feedback at design time and runtime

Thanks to this approach, the development of HSI applications fulfilling both functional and non-functional requirements will now have application and platform concerns isolated from each other. Additionally, the refinement of KPIs will be either completely automatic at runtime or profiled at design time so as to provide the developer with enough information to make the necessary changes. 


\subsection{Objectives}

The main objective of this $\mathrm{PhD}$ manuscript [OBJ-P] is to propose a methodology -and its associated toolchain- capable of optimizing dataflow applications on multi-/manycore architectures considering energy as the main non-functional requirement. In order to develop this methodology, the following starting point is considered:

- Application: an HSI cancer detection processing chain discerning between tumorous and healthy tissues in neurosurgical and dermatological fields will be employed. This application is composed by a set of algorithms that presents diverse parallelization natures and different computational requirements.

- Architecture: an embedded HPC many-core architecture with a low-level energy consumption called Multi-Purpose Processor Array (MPPA-256-N manufactured by Kalray S.A. will be employed in this study. This architecture presents different memory hierarchies and will provide diversity in terms of processing resources and parallelization opportunities.

- Application prototyping environment: a dataflow-based Y-chart design flow that supports both design time and runtime refinements will be selected.

Finally, in order to define and evaluate the Y-chart dataflow-based optimization loop, 4 secondary objectives have been settled:

- [OBJ-S1] Use-case implementation: regarding the system that will be used to develop and characterize the optimization loop, the reference use-case has to be implemented first manually (as a baseline) and then using a dataflow approach (to apply the methodology).

- [OBJ-S2] Monitoring infrastructure: an approach that combines the benefits of PAPI library and the abstraction of Y-chart design flow will be proposed. This infrastructure should be able to characterize dataflow applications based on hardware performance information at runtime.

- [OBJ-S3] KPI estimation: a KPI estimation procedure compatible with the proposed toolchain and based on information that can be retrieved during application execution will be built. In this $\mathrm{PhD}$, the selected KPI is the energy consumption, so a methodology to model the platform energy consumption will be sought. This modeling approach will stress the idea of generating an application-agnostic model.

- [OBJ-S4] Energy-awareness: to include energy-awareness in the system, guided or automatic optimization will be included within the implementation of design time and runtime feedback loops. To be precise, an energy-aware decision making mechanism that adapts the system considering both the monitoring data and the associated KPI information will be required. 


\subsection{Thesis layout}

This document is organized in 8 Chapters. First, in Chapter 1, a brief introduction composed of the motivation and the objectives of the $\mathrm{PhD}$ is presented.

Secondly, Chapter 2 introduces the necessary concepts and technologies to contextualize the rest of the ideas presented in this document.

Chapter 3 discusses the state of the art and defines the energy-aware Y-chart dataflow-based design methodology proposed in this $\mathrm{PhD}$. The different decisions are taken based on the requirements extracted from the analysis of the selected use-case: an HSI cancer detection processing chain running on the MPPA platform.

Chapter 4 deals with the aspects associated with the monitoring block of Figure 1-1 [OBJ-S2]. To be precise, the so-called PAPIFY monitoring infrastructure is detailed, focusing on the dataflow oriented abstraction level that characterizes the tool and that differentiates it from PAPI library.

In Chapter 5 the energy consumption modeling methodology is described and, alongside the explanation, a model of the MPPA platform is built. Since the KPI to be optimized in this $\mathrm{PhD}$ is the energy consumption, this model will be used in the KPI estimation block of Figure 1-1 to estimate the system energy consumption [OBJ-S3].

Then, Chapter 6 presents the energy-aware decision making protocol that will be used to select the most efficient system configuration based on the available profiling data [OBJ-S4]. After this, both design time and runtime energy-aware optimization loops proposed in this $\mathrm{PhD}$ are detailed in this chapter [OBJ-P]. Specifically, at design time, the DSE protocol is enhanced with energy-awareness, while on-thefly optimizations are embedded within a runtime resource manager. This chapter covers the information related to both runtime decisions block of Figure 1-1 and the connection between KPI estimation and user-defined constraints blocks.

Chapter 7 gathers the results obtained during the validation of the methodology proposed during this PhD [OBJ-P]. Specifically, by means of a functional validation of the HSI cancer detection processing chain, the system is implemented by hand as a baseline to compare with [OBJ-S1]. Then, a characterization of the benefits obtained when applying the methodology proposed to automatically reduce the energy consumption is performed. To be precise, independent analyses are done for the design time and the runtime optimization loops using a dataflow version of the HSI cancer detection processing chain [OBJ-S1].

Finally, in Chapter 8, some conclusions are extracted from the work presented in this document and, additionally, some future research lines are proposed. Lastly, the impact of this work is deeply analyzed, highlighting the publications, the participation on different projects, the collaborations carried out during the development of this $\mathrm{PhD}$, the grants received and the awards obtained. 



\section{STATE OF THE ART}

Once the motivation behind the development of this $\mathrm{PhD}$ and its associated objectives have been introduced in Chapter 1, this chapter studies the state of the art of the concepts that are required to contextualize the ideas presented in this $\mathrm{PhD}$. Specifically, an independent analysis is performed for of each module composing the Y-chart design methodology where the system energy consumption is iteratively optimized.

In this regard, the aspects related to the use-case that motivated this work-energyefficient $\mathrm{HSI}$ tumor detection implementations on neurosurgical and dermatological fields- are analyzed. First, the existing HSI applications targeting cancer detection are surveyed in Section 2.1. Then, Section 2.2 explains the necessity of embedded HPC architectures and presents some examples of this type of platforms.

After this, a survey of the different design methodologies existing in the state of the art is included in Section 2.3. This study has been performed considering the developer's effort, starting from low-level approaches requiring a lot of effort and finishing with automatic multi-objective design methodologies.

Finally, the ideas associated to the 3 blocks composing the energy consumption optimization loop are reviewed, namely: monitoring, energy modeling and energyawareness. Section 2.4 gathers an analysis of tools related to application characterization considering different levels of abstraction. In Section 2.5, the state of the art linked to the modeling of the platform energy consumption is studied, addressing aspects like the definition of the model, the types of benchmarking and the strategies used to infer a model from these data. Lastly, a set of techniques that can be applied to reduce the energy consumption of a system is analyzed in Section 2.6.

\subsection{HSI cancer detection applications}

During this section, a brief overview of the most relevant uses of HSI technology in cancer detection applications is presented. This review summarizes the main ideas found in [Halicek'19a], in which all the applications are detailed. As mentioned in [Halicek'19a], the applications can be categorized by 1) the organ being analyzed, 2) the experiment being ex-vivo or in-vivo and 3) the subject being an animal or a human being. Figure 2-1 summarizes the different domains presented in this part section. 


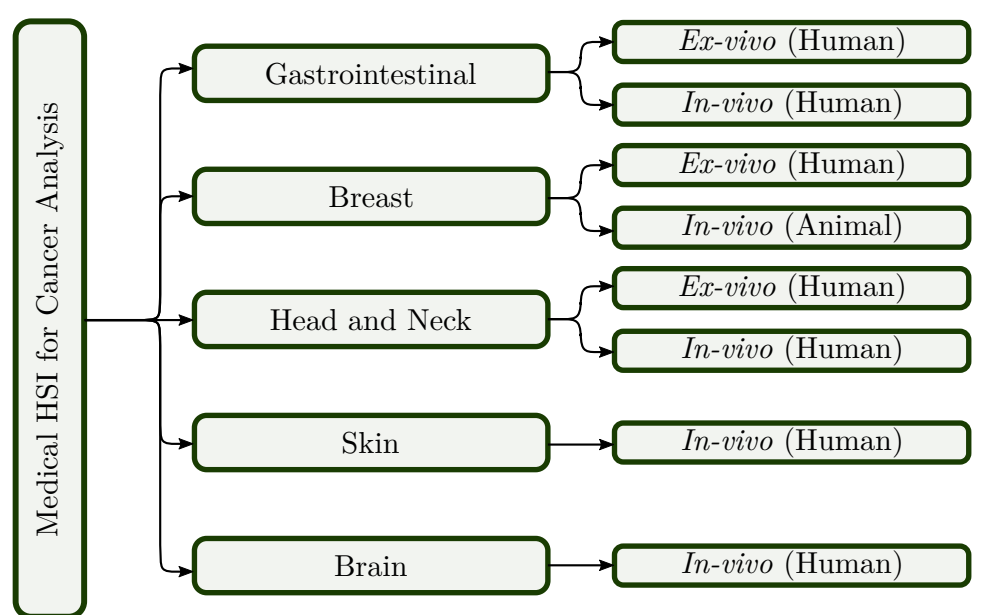

Figure 2-1: HSI technology applied to cancer detection. Based on image from [Halicek'19a]

\subsubsection{Gastrointestinal}

Gastric carcinomas are the second leading cause of cancer death globally. On top of that, when the disease is diagnosed locally advanced or metastatic, surgical resection is considered the only curative therapy Orditura'14. Currently, these surgeries are performed through laparoscopic guided by endoscopy [Pascual'16]. As a drawback, with this procedure, surgeons suffer of a lack of tactile feedback, which can be replaced with HSI information.

Concerning the ex-vivo experiments, Baltussen'19] presents a work to provide the surgeon with the missing information in laparoscopic surgeries. This system was able to distinguish among healthy colorectal mucosa, normal fatty tissue and adenocarcinoma. In this case, a Support Vector Machine (SVM) classification has been used, reaching a $93 \%$ of patient-level accuracy.

In [Han'16], an HSI colonoscopy system is employed to differentiate between normal colonic mucosa and colorectal tumors in human patients. In this study, a $95 \%$ of accuracy is obtained by means of a recursive divergence algorithm to reveal the most relevant wavelengths in the images.

\subsubsection{Breast}

In breast cancer surgery -known as lumpectomy-, one of the main objectives is to conserve the breast and complement this surgery with radiation therapy to completely remove the tumor. Nowadays, around $20 \%$ of women who undergo lumpectomy require additional surgeries, as they are diagnosed positively during the post-operative diagnosis [Jacobs'08]. In this case, HSI guided intra-operative biopsies can help the surgeon to reduce this figure.

A study carried out on human ex-vivo samples is presented in [Pourreza-Shahri'13]. In this study, an accuracy of $99.38 \%$ is reached when applying an SVM classifier 
together with a feature extraction and a dimensional reduction to differentiate between cancerous and non-cancerous tissues.

Additionally, considering in-vivo experiments, |Panasyuk'07| is one of the most relevant works. In this work, intra-operative surgery is performed to 60 rats affected by an induced breast cancer. As a result, $89 \%$ and $94 \%$ of sensitivity (correctly identified positives) and specificity (correctly identified negatives) rates are obtained, respectively, when classifying the scenes to distinguish tumor, blood vessels, muscle and connective tissues.

\subsubsection{Head and neck}

Among the head and neck cancers, which, according to |Vigneswaran'14|, are the $6^{\text {th }}$ most common cancer worldwide, the $90 \%$ of the ones affecting the upper aerodigestive tract are squamous cell carcinomas. Although this type of cancer is mainly treated with surgical resection, almost a $20 \%$ of patients post-diagnosed negatively (tumor properly resected) suffers from cancer recurrence. This fact could be minimized by means of using HSI during surgery to validate the tumor resection with near-real-time information [Halicek'19a].

Considering ex-vivo experiments, [Fei'17, Lu'17] demonstrate that the use of Linear Discriminant Analysis (LDA) outperforms both reflectance-based imaging techniques and different machine learning classification approaches. Specifically, these studies are based on distinguishing cancerous and non-cancerous tissues from oral cavity and thyroid samples. Additionally, in [Halicek'19b], HSI is proven to be useful in optical biopsies when it is combined with a Convolutional Neural Network (CNN) algorithm.

Working on in-vivo environments, [Jayanthi'11] presents a work related to detecting malignant changes in the oral cavity. In this work, applying both a Principal Component Analysis (PCA) to reduce the dimensionality of the problem and an LDA to classify the data, sensitivity and specificity rates above $95 \%$ are obtained. Furthermore, in 2016, [Regeling'16] presents a study of laryngeal cancer where a Random Forest RF machine learning approach is used, reaching an overall accuracy of $88 \%$.

\subsubsection{Skin}

According to the American Cancer Society, skin cancer incidence trend has been increasing for the last 30 years. Additionally, this type of cancer can be usually divided into non-melanoma skin cancer and melanoma. The former is the most frequent, but, on the contrary, the latter is the most deadly [Johansen'20]. Even if this cancer is currently being tackled using RGB cameras and dermoscopic imaging, according to [Johansen'20|, there is a growing interest on using HSI cameras to deal with it.

Considering that the treatment of melanoma skin cancer consists of removing the disease as soon as it is identified as malignant, HSI could help in the early 
diagnosis maintaining the non-invasive and non-ionizing advantages of previous imaging technologies. Currently, the two main trends relating skin cancer detection and hyperspectral imaging are feature extraction and spectral band selection. The former covers from hand crafted features inspired by the ABCD (asymmetry, border, color and differential structures) rule of dermoscopy [Nachbar'94] like [Stamnes'17] to PCA, where the spectral bands are combined to reduce the spectral redundancy [Kazianka'08. The latter, on the contrary, focuses on selecting the most relevant bands in the spectrum so as to minimize the computational complexity of the image classification. For example, in both [Zherdeva'16] and [Lorencs'16], this strategy is applied and they present promising results speeding up both training and inference without reducing the classification accuracy.

Nevertheless, in [Esteva'17], it has been demonstrated that the combination of both spectral and spatial information would be the ideal approach in the skin cancer detection field. Even if this work has been carried out using RGB images, its conclusions can be extended to foresee a system combining both the ABCD features with the enriched spectral information providing even more promising results in this field. This technique has been successfully applied in, for instance, remote sensing applications using HSI Makantasis'15, Mughees'17].

\subsubsection{Brain}

Brain tumors cause between 2 and $3 \%$ of the deaths due to cancer worldwide Halicek'19a|. The main issue with this type of cancer is that neurosurgeons not only need to completely remove the tumor -minimize tumor recurrence [Petrecca'13]-, but also try to reduce as much as possible the removal of healthy brain -maximize patient's quality of life [Stummer'11].

This accurate margin delimitation is currently addressed using different image guidance technologies:

1. Intra-operative neuro-navigation: can be used in real-time but is affected by brain shifting [Gerard'17], which is the phenomenon of the brain being displaced after craniotomy and durotomy.

2. intra-operative Magnetic Resonance Imaging (iMRI): requires special equipment in the operating theater and increases the surgery duration in 20 to 75 minutes per image taken [Chowdhury'18.

3. intra-operative Ultrasound (iUS): is unaffected by brain shifting and provides results in real-time but it can lead to the resection of normal parenchyma Chacko'03, i.e., the cells that perform the organ function.

4. 5-ALA is (only) capable of identifying boundaries of high-grade tumors but it has the important drawback of being invasive [Stummer'06]. 
In order to avoid all these problems, HSI technology applied to in-vivo environments has been developed in a European project called HypErspectraL Imaging Cancer Detection (HELICoiD). Its main goal is to develop a methodology to distinguish between healthy and tumorous tissues in real time during neurosurgical procedures [Fabelo'16b, Salvador'17]. This project is analyzed in Subsection 2.1.6.

\subsubsection{HELICoiD}

HELICoiD project was an interdisciplinary work at the edge of the biomedical domain funded by the European FP7 Future and Emerging Technologies (FET) Open. The main goal of this was to develop a prototype capable of accurately identifying the tumor boundaries during neurosurgical procedures following a non-invasive and non-ionizing approach, i.e., through HSI [Salvador'17]. Additionally, HELICoiD also aimed at creating the first open access HSI database for in-vivo brain tumor images [Fabelo'19b].

In this sense, the technical challenge of the project consisted of analyzing the huge amount of data retrieved by the HSI system and, on top of that, providing a response in real-time. Achieving such performance is the only way the system becomes useful to the surgeon in an operating theater environment. Considering this scenario, the use of computational accelerators is considered unavoidable [Salvador'17].

From the algorithmic point of view, the proposed processing chain, shown in Figure 2-2, is a hybrid classification approach where both supervised and unsupervised classification techniques are combined. To be precise, a preprocessing stage is applied first to the raw image, shown in Figure 2-3a, so as to calibrate the image and remove spectral redundancies [Fabelo'16a]. After reducing the spectral information to 128 bands, both supervised and unsupervised classifications are independently applied to the image. The former aims at associating a label, which can be healthy tissue (in green), tumor tissue (in red), hyper-vascularized tissue (in blue) or background (in black), by means of a model previously trained using a golden reference provided by the neurosurgeons. As a result, after applying a spatial-spectral classification, a colored map is presented, as can be seen in Figure 2-3b. The latter, on the contrary, clusters the pixels of the image considering their similarity and without any previous training. In this case, as shown in Figure 2-3c, a set of unlabeled clusters is obtained. Finally, in order to enhance the result of the classification, both supervised and unsupervised classification results are combined providing, thus, a hybrid classification map exemplified in Figure 2-3d. In this final map, the unlabeled clusters are correlated with the supervised classification result to link each cluster with one of the aforementioned tissues, opening up the possibility for neurosurgeons to clearly identify the tumor tissues boundaries.

In order to cope with this algorithmic complexity and considering the real-time constraint existing within the requirements of HELICoiD project, part of the processing chain has been accelerated using a many-core architecture called MPPA-256-N, manufactured by Kalray S.A. In this sense, the supervised classification algorithm 


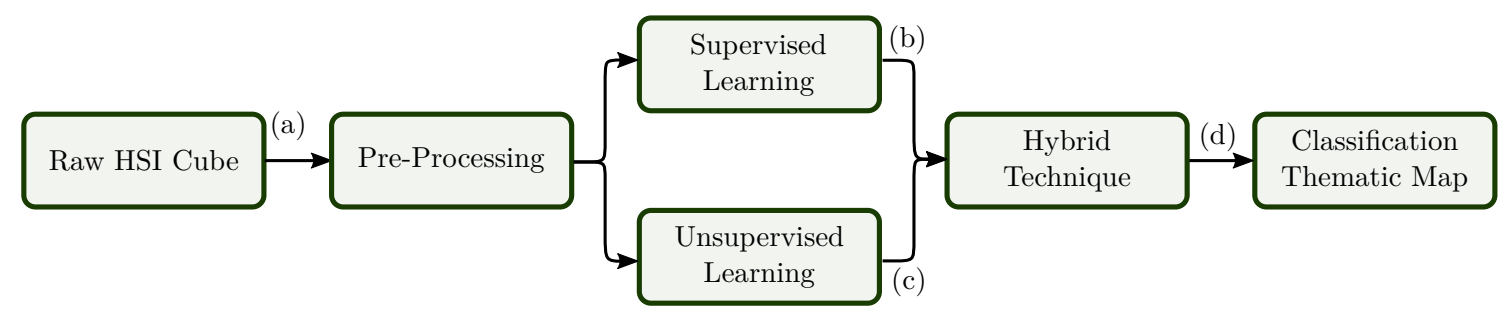

Figure 2-2: HELICoiD processing chain

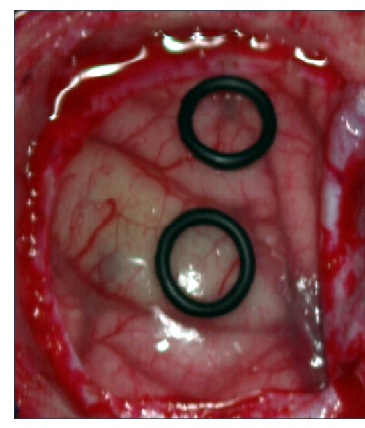

(a) Raw image

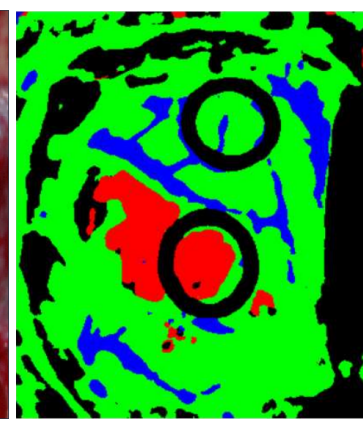

(b) Supervised classification

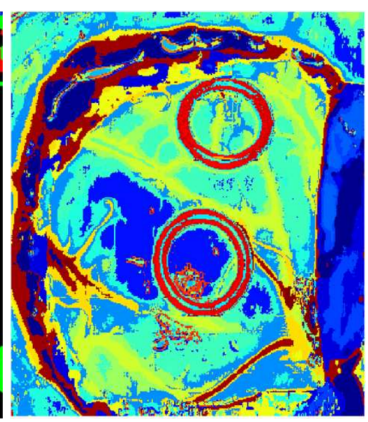

(c) Unsupervised classification

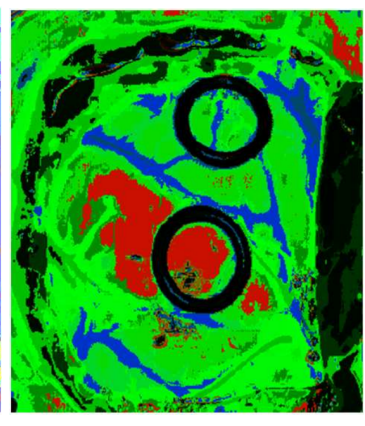

(d) Hybrid classification

Figure 2-3: HELICoiD processing chain partial results. Based on Salvador'17

has been selected to be the one accelerated, as it is the one requiring the most computational resources [Fabelo'18b]. With this setup, the overall accuracy of the system reaches values over $99 \%$ and the images are processed in 1 minute, which, from neurosurgeons point of view, can be enough to help them during the surgery.

\subsection{High performance embedded computing}

The large amount of information composing hyperspectral images combined with the application complexity, which currently demands real-time processing responses, drastically increases platform performance requirements. Additionally, according to Moore's law [Moore'65], the number of transistors that can fit in a chip is doubled every 2 years. This trend has become true [Thompson'06] due to the minimization of the transistors, which, in turn, allows the frequency of the processors to be increased. However, the drawback is the counterpart increment of the power/energy required to make these resources work, hence increasing the benefits obtained with low energy consumption implementations.

In order to cope with this effect, platform manufacturers started working on increasing the number of processors available in a single chip, creating, thus, the first 
multicore processor. By doing so, the processors power consumption is kept among reasonable levels and the computational capabilities are increased. On the contrary, problems related to the data management among processors appear.

In this line, both Graphics Processing Units (GPU $)$ and many-core architectures have gained popularity since the required development effort is, in general, lower than the one required to develop specific solutions on Field Programmable Gate Arrays (FPGAs) Torun'16]. On the one hand, GPU solutions usually yield the most competitive results in terms of speedups and processing times. On the other hand, many-core approaches normally provide more energy efficient solutions.

\subsubsection{GPU platform}

GPU were born during the 80s to ease the computation of graphics applications [Owens'08]. For this reason, these co-processors gather a big amount of simple and small cores coupled with small control units. This structure provides all the cores with the capability of executing the same instruction on different data, which is usually known as Single Instruction Multiple Data (SIMD).

The general structure of GPUs is displayed in Figure 2-4 and composed of a set of Streaming Multiprocessors $(\overline{\mathrm{SM}} \mathrm{s})$ containing several cores communicating through a Shared Memory (SMEM) and a local cache memory. In turn, each SM is in charge of accessing the global GPU memory, normally a Double Data Rate (DDR) memory. GPUs are usually divided into desktop and embedded platforms [Lazcano'19b].
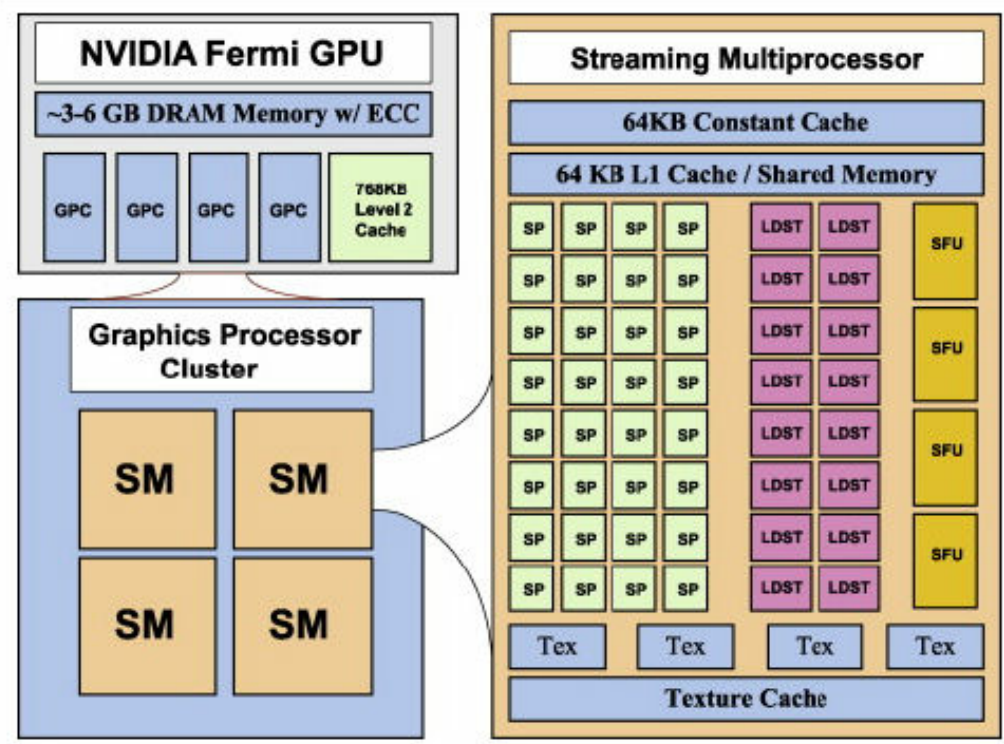

Figure 2-4: GPU general structure. Image extracted from Reyes'13

Desktop GPU 3 are characterized for having thousands of cores working in parallel with huge Graphics DDR GDDR memories, in the order of GB. However, these GPUs present high power consumptions. Some examples from NVIDIA are: 
Tesla K40: 2880 cores working at $0.875 \mathrm{GHz}$ with a 12GB GDDR 5 memory. This platform has an average power consumption of $33 \mathrm{~W}$.

GTX 1060: 1280 cores working at $1.78 \mathrm{GHz}$ with a 6GB GDDR 5 memory. This platform has an average power consumption of $28 \mathrm{~W}$.

RTX 2080: 2994 cores working at $1.71 \mathrm{GHz}$ with a 8GB GDDR 6 memory. This platform has an average power consumption of $71 \mathrm{~W}$.

Regarding embedded GPUs, they normally contain some hundreds of cores and, additionally, the type of memory used is Low Power DDR (LPDDR), which has a lower bandwidth when compared to the previous ones. Consequently, even though their computational resources are considerably lower, their power consumption is, in turn, also drastically reduced. This trade-off is useful when considering, for instance, applications where portable devices are required. Two examples of these platforms are:

Jetson TX1 |Aragón'19]: 256 cores working at $0.998 \mathrm{GHz}$ with a 4GB LPDDR4 memory. This platform has an average power consumption of $10 \mathrm{~W}$.

Jetson TX2 [Lazcano'19b]: 256 cores working at $1.3 \mathrm{GHz}$ with a 8GB LPDDR4 memory. This platform has an average power consumption of $5.4 \mathrm{~W}$.

\subsubsection{Many-core architecture}

Many-core architectures can be considered an extension of multi-core processors. Specifically, many-core architectures are characterized by grouping cores into compute units and communicating those compute units through a Network-on-Chip (NoC). When compared to multi-core processors, many-core architectures present two main differences: 1) while multi-cores usually provide full cache memory coherency, many-cores may not extend it beyond the compute cluster and 2) a multi-core normally scales replicating its cores and, on the contrary, many-cores scale replicating their compute clusters. Consequently, the scalability of many-core ranges from hundreds to thousands of cores.

A clear example of a many-core architecture is the MPPA-256-N [Dinechin'13] manufactured by Kalray S.A. This Non-Uniform Memory Access (NUMA) architecture is a single-chip homogeneous Multi-Processor System on Chip (MPSoC) composed of 256 Very Long Instruction Word (VLIW) Processing Elements (PEs) distributed among 16 Compute Clusters (CC 3 ), whose working frequency can vary between 400 and 600 MHz. This platform has an average power consumption of 15W[Lazcano'19b]. As shown in Figure 2-5, all $\mathrm{CC} s$ are interconnected through two $\mathrm{NoC} \beta$, one for control (C-NoC) and another one for data communications (D-NoC) [Roux'16]. In addition, external memory communications are handled with two quad-core Input/Output Subsystems (IOs). Each of them also contains 4 Direct Memory Accesss (DMAs), onchip memory and a DDR controller to access up to 4 GB DDR memory banks. Finally, each IOsubsystem runs either ClusterOS [Hascoet'18] Operating System (OS) or Linux. This structure is shown in Figure 2-6. 
Concerning CCs, each of them gathers $16 \overline{\mathrm{PE} s}$ and a Resource Manager (RM) core responsible for running the ClusterOS [Hascoet'18] OS and handling events and interruptions. They also contain a $2 \mathrm{MB}$ of SMEM, providing a bandwidth of $38.4 \mathrm{~GB} / \mathrm{s}$, and a DMA to communicate with the $\mathrm{IO}$ s through the NoC.

Finally, the inner structure of both the $\mathrm{PE} s$ and the $\mathrm{RM}$ cores implements a 32bit 5-way VLIW architecture, which includes a multiply and accumulate floating-point unit, two arithmetic and logic units, a load/store unit and a branch and control unit. Furthermore, each PE also includes a Memory Management Unit (MMU) for virtual memory support and process isolation.

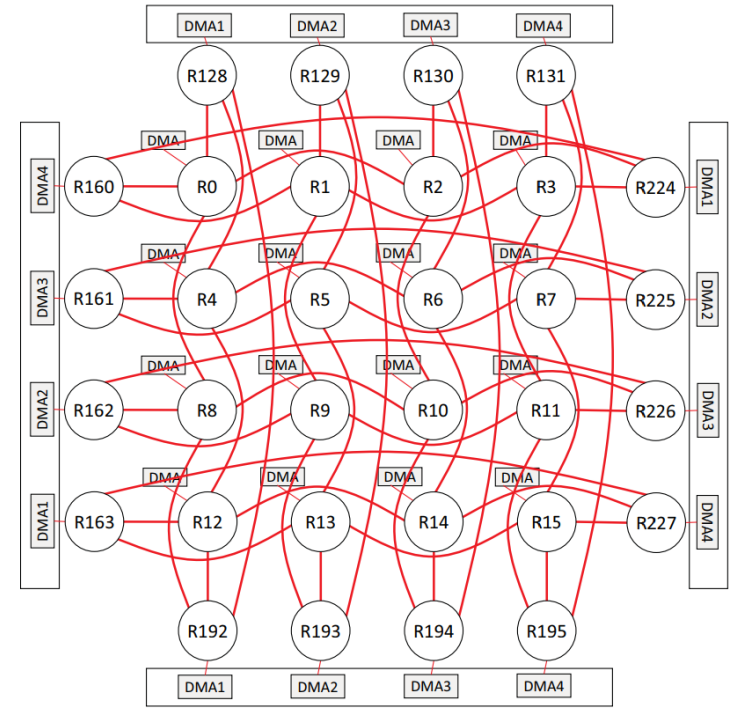

Figure 2-5: MPPA $\mid$ NoC, Image extracted from Maruyama'17

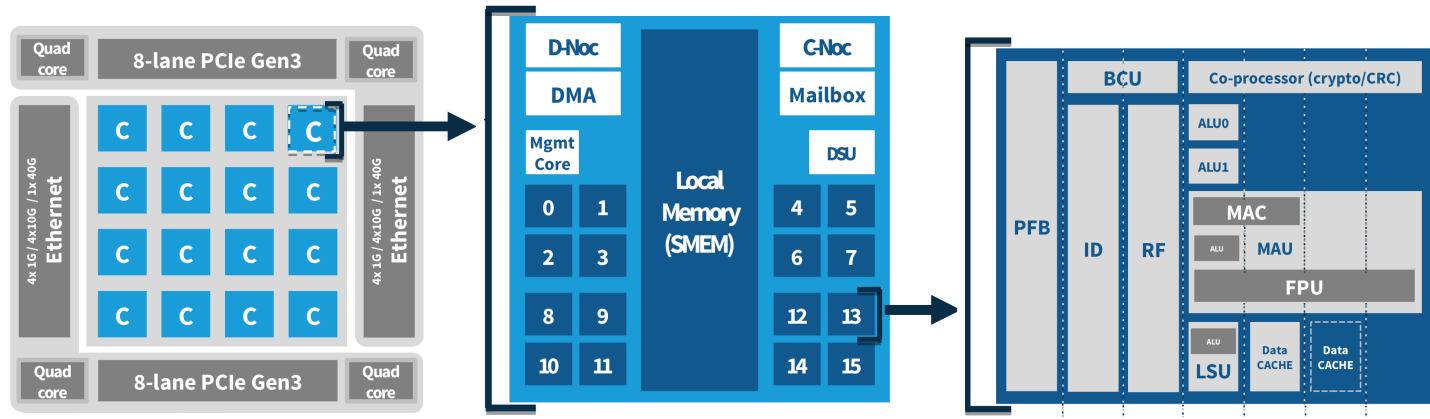

(a) Platform

(b) Compute Cluster $\mathrm{CC}$

(c) Processing Element (PE)

Figure 2-6: MPPA-256-N Bostan structure 


\subsection{Application design methodologies}

During the last few decades, the complexity of both applications and architectures have increased enormously. This trend has been tackled by developing methodologies, languages and tools aiming at reducing the effort required by the developers to have an efficient implementation in terms of application performance on multi-/many-core architectures. In this line, a brief survey of the different approaches existing within the literature is presented in this section. First, different Application Programming Interfaces $(\overline{\mathrm{API}} \mathrm{s})$, whose objective is to explicitly program the application parallelism, are analyzed. Secondly, a set of Computer Aided Design (CAD) tools, whose objective is to automatize parallelization and optimization steps, are presented. After that, tools based on dataflow MoCs, where processing and data dependencies are explicitly expressed by the developer, are introduced. Finally, design methodologies supporting multi-objective optimizations are explained. These methodologies consider not only latency when building a system, but also aspects like power consumption or resilience.

\subsubsection{Parallel programming APIs}

In this part of the analysis, different APIs are considered. These libraries were born from the necessity of implementing applications that take advantage of the parallelism opportunities offered by current multi-/many-core architectures.

Portable Operating System Interface (POSIX) threads [Butenhof'97], also known as pthreads, is a parallel execution model standardized by Institute of Electrical and Electronics Engineers (IEEE) that allows developers to control multiple working procedures that overlap in time. These procedures are known as threads and are used to distribute the work among the different processing elements in a multi-/many-core architecture. In order to unify the different implementations of the threads concept, pthreads has been defined as a C API and its associated library. The use of this library ensures the code compatibility among the different supported OS. This library serves as a basis for the development of more complex parallel programming APIs, and it is commonly used to introduce engineers to task-parallelism and data-parallelism concepts [Curtsinger'19], as the code executed by each procedure/thread needs to be explicitly coded by the developer. As an example, in Figure 2-7, a basic usage of pthreads is shown. In this example, a master thread creates 2 working threads and waits until they finish their jobs, which are performed in parallel, to continue the execution.

To ease the code parallelization, Open Multi-Processing (OpenMP) was created by the end of the 90s |Dagum'98|. This new standard is composed of a set of directives and function calls, allowing the developer to easily parallelize shared-memory applications when they are implemented on Fortran, $\mathrm{C}$ or $\mathrm{C}++$. OpenMP behavior is, in general lines, based on defining sequential and parallel regions inside the application code and, when including these directives, the work contained in the parallel regions is 


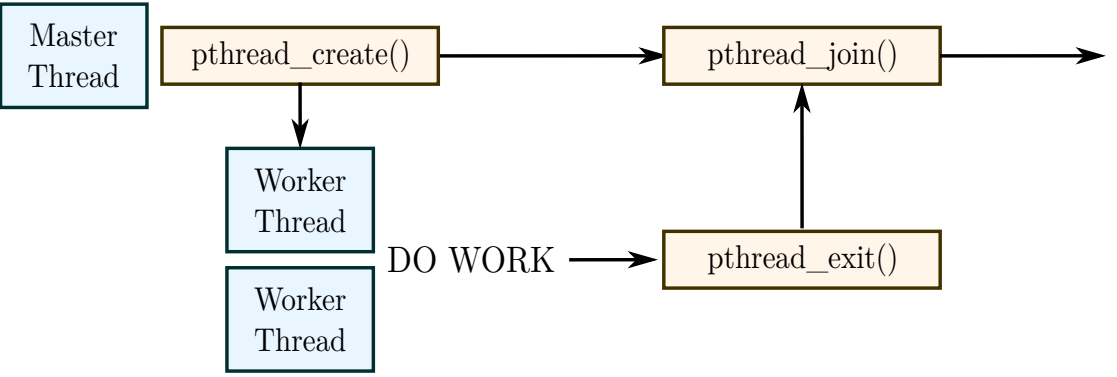

Figure 2-7: Basic example of pthreads usage

automatically distributed among the available threads using, normally, a fork/join strategy [Chandra'01], as shown in Figure 2-8, One of the main characteristics of this approach is that the workload distributed among cores has to be uniform, which means that all the threads will execute the same code but no multitasking is supported.

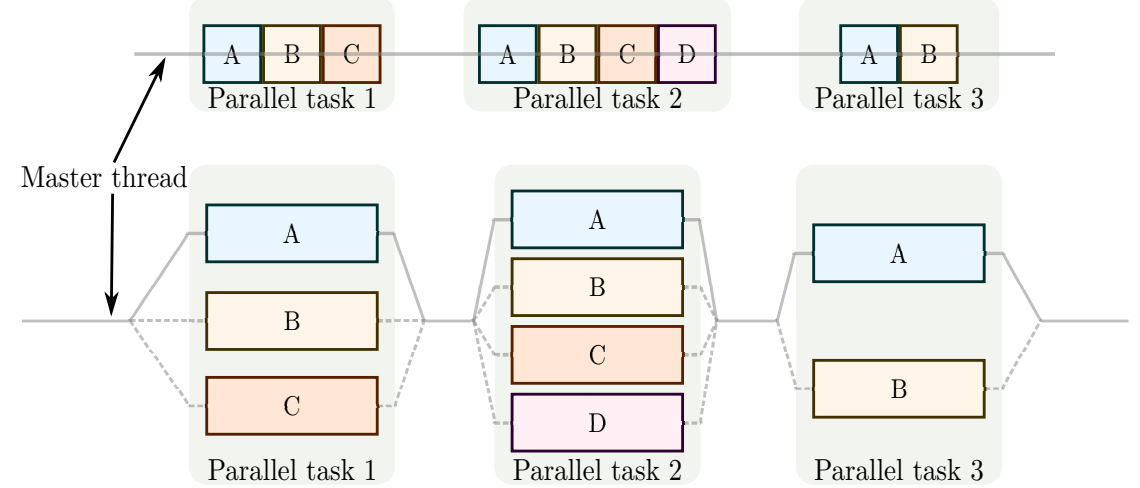

Figure 2-8: Basic example of OpenMP fork/ join usage

Open Computing Language OpenCL ${ }^{1}$ is a framework standardized by Khonos international consortium in 2013. This framework appeared to deal with heterogeneity in both architectures and code to be parallelized. In this sense, OpenCL controls the flow of work using a host, usually a Central Processing Unit (CPU), that dispatches tasks among the available accelerators, which can be other CPUs, GPUs or FPGAs. By doing so, developers are provided with a framework capable of using, at the same time and for the same application, resources of different nature and, consequently, they can also select the most suitable $\mathrm{PE}$ when distributing the workload. In order to support this variability, OpenCL has its own programming language and each vendor is usually responsible of supporting it so as to include their architectures within the available resources. It should be noted that the OpenCL programming model is based on Compute Unified Device Architecture (CUDA framework by NVIDIA.

1 https://www.khronos.org/opencl/ 


\subsubsection{Computer aided design}

Secondly, a plethora of tools appeared among the literature aiming at reducing developers' effort in the sense of parallelism extraction and definition. The objective of these tools is to automatically parallelize sequential code and, thus, prevent the developer from this task. In this section, a selection of tools targeting embedded systems is presented.

In the case of MPSoCApplication Programming Studio (MAPS) |Ceng'08], it takes a sequential code as an input and transforms it into a parallelized application through 3 steps: analysis, partitioning and code generation. First, this tool analyzes the code so as to extract data and control information that is extrapolated to task-, data- and pipelinelevel parallelism. After this, MAPS associates the parallelism of the application with the parallelism possibilities of the target architecture, whose description is included as an input considering the cost of performing additions, multiplications, communication, etc. Finally, this tool is capable of generating the associated parallelized code based on the Tightly-Coupled Thread (TCT) execution model [Isshiki'08], which is a simple way of expressing the sequential code in concurrent tasks. This TCT code will require the use of a TCT compiler that will, first, parse the code to standard C and, after that, generate the application binaries.

Parallelism Extraction for Embedded Systems (PaxES) is a framework targeting multi-core systems that puts together 3 parallelism extraction methods: 1) Integer Linear Programming (ILP) to extract task-level parallelism [Cordes'10, 2) ILP for pipeline-level parallelism [Cordes'11] and 3) multi-objective extraction of task-level parallelism using genetic algorithms [Cordes'12]. By combining these tools, a multiobjective framework where the designer is free to choose the desired trade-off among execution time, energy consumption and communication load in an embedded system is provided.

The Architecture oriented paraLlelization for high performance embedded Multicore systems using scilAb (ALMA) project [Goulas'13], funded by the European Commission under grant number FP7-ICT-287733, presents a solution to optimize applications from Scilab to embedded MPSoC code. As explained in [Sorel'05a], Scilab targets high-level, numerically oriented programming language that cannot be considered purely dataflow, as it mixes both control and data in its specification. ALMA project toolchain first translates the Scilab code to $\mathrm{C}$ code that is then loaded into the Generic Compiler Suite GeCoS compiler [Floc'h'13. Although this interactive compiler originally targeted custom hardware design using High Level Synthesis (HLS) where not only speed was optimized, but also area and accuracy, within the context of ALMA project, it was extended to MPSoC architectures [Stripf'13]. In this line, two extensions were applied: 1) a coarse-grain parallelism extraction and optimization step to distribute application tasks considering the multi-core architecture constraints and 2) a fine-grain parallelism extraction step to exploit SIMD instructions available in modern MPSoC processors. 


\subsubsection{Dataflow based design}

Following the idea of automatically generating parallel code, a set of dataflowbased design tools appeared in the literature. Dataflow paradigm [Ackerman'79] is a specific category within MoCs where data communication and processing are explicitly isolated in the application definition. Approaches based on this idea benefit from the high level of abstraction and architecture independence when developing applications, which also facilitates automatic DSE [Pimentel'16].

By the end of the 90s, Grandpierre'99 introduced Synchronous Distributed Executive (SynDEx) tool, which is a proprietary tool based on Algorithm Architecture Matching (AAM) Y-chart prototyping methodology, represented in Figure 2-9, In this methodology, the architecture definition is completely isolated from the application design and, after that, the system is implemented ensuring that user-defined constraints are satisfied. From the point of view of the application design, a Direct Acyclic Graph (DAG) is built to represent infinite iterative applications using a repetition pattern where the vertexes represent application operations and the edges correspond to the data dependences. In the case of the architecture, the representation is essentially a set of operators (computation resource) interconnected through communicators (e.g., a DMA) accessing to memory resources (either Random Access Memory ( $(\overline{R A M})$ or Sequential Access Memory $(\overline{\text { SAM }})$ ) and the buses employed to communicate among them. Finally, during the implementation step, the main objective is to ensure that real-time constraints are satisfied and, thus, the mapping/scheduling algorithm focuses on optimizing latency [Sorel'05b].

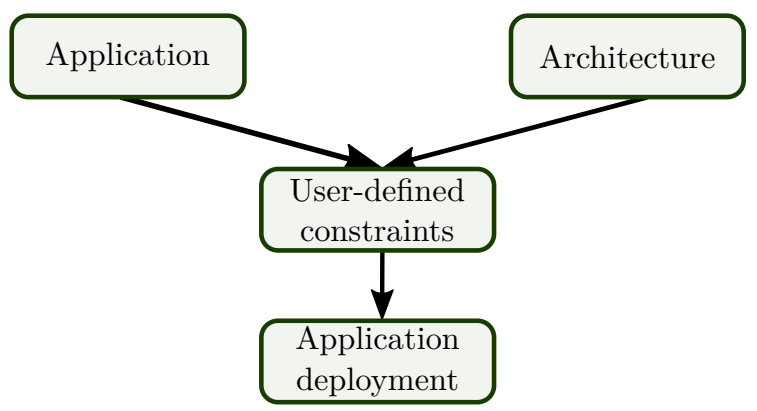

Figure 2-9: Y-Chart design block diagram

ORCC [Yviquel'13b] is an open-source dataflow-based design framework that can generate parallelized applications in a variety of languages, e.g., C, HLS or LLVM. Concerning the application design, this tool uses Reconfigurable Video Coding (RVC)Cal Actor Language (CAL) dataflow language and the Dataflow Process Network (DPN) dataflow MoC, Using this approach, non-deterministic applications can be modeled, as the so-called actors, which are the computation blocks in which the application is divided into, can be fired as soon as they have enough input data and generate more or less output data tokens depending on the input data they receive. To complement this environment, TURNUS Casale-Brunet'13 provides it with DSEcapabilities. However, this approach cannot ensure neither deadlock-freeness nor memory boundedness. 
PREESM is an open-source dataflow-based Y-chart prototyping tool [Pelcat'14a] where both application and architecture are defined through models. On the application side, Parameterized and Interfaced Synchronous DataFlow (PiSDF) dataflow $\mathrm{MoC}$ is used to define the application graph. This model extends standard Synchronous DataFlow (SDF) MoC [Lee'87], where, actors are represented by nodes communicating among them through First-In First-Out queues (FIFOs). Specifically, the extension consists on introducing hierarchical graphs using interfaces and parameterized FIFO sizes. On the architecture side, System-Level Architecture Model S-LAM is used to describe parallel architectures as a set of PEs transmitting data through communication nodes and data links. The implementation is carried out under a set of user-defined constraints and, taking advantage of the analyzability of the PiSDF MoC, deadlock freeness is ensured by construction in the automatically generated code. It should be noted that this tool not only provides compilable code to test the system, but also simulates its execution to enhance system design.

As an extension of PREESM framework, a runtime resource manager called Synchronous Parameterized and Interfaced Dataflow Embedded Runtime (SPiDER) was developed to support runtime reconfiguration of PiSDF dataflow applications [Heulot'14]. This new tool uses the same framework but, in this case, dynamic parameters can be defined in the graph. To be precise, the application is managed by a Global Run-Time (GRT) manager that is in charge of distributing the application actors among the different $\mathrm{PE} \beta$. These $\mathrm{PE}$, also called Local Run-Times (LRTs), are in charge of executing the actors and, if necessary, send back the new values of the dynamic parameters to the GRT, which will redistribute the application accordingly.

\subsubsection{Multi-objective optimization}

Finally, considering the current trend existing within the application requirements, where multiple requirements need to be fulfilled at the same time, new design methodologies are being developed in the context of collaborative projects. Two examples are introduced in this analysis: one optimizing the application at design time using a multi-objective DSE based on [Pimentel'06]; and the other enabling system self-awareness to support runtime application auto-tuning [Palumbo'19a $\mid$.

In the case of software framework for runtime-Adaptive and secure deep Learning On Heterogeneous Architectures (ALOHA) European project, which has received funding from the European Union's Horizon 2020, under grant agreement No. 780788, the main objective is to facilitate the implementation of Deep Learning (DL) algorithms on heterogeneous low-power architectures [Meloni'19]. To be precise, this project proposes a tool flow to reduce development costs by automating several design steps. First, the Deep Neural Network $(\overline{D N N})$ design is guided through DSE where all the generated points are analyzed in terms of accuracy, robustness, inference, performance and power consumption. Once the algorithm is optimized, its partitioning and mapping on the heterogeneous low-power architecture is also automated by means of a System-level DSE engine. This process is carried out 
using a genetic algorithm [Pimentel'06] and selects the most efficient mapping considering throughput, latency and power consumption. Finally, the application code is automatically generated considering the target hardware architecture and reducing, when possible, power consumption through the use of power gating, clock gating or Dynamic Voltage and Frequency Scaling (DVFS). These power consumption reduction techniques are thoroughly explained in Section 2.6.

Cross-layer modEl-based fRamework for multi-oBjective dEsign of Reconfigurable systems in unceRtain hybRid envirOnments CERBERO Palumbo'19a European project, funded by the European Union's Horizon 2020, under grant agreement No. 732105, targets the Cyber-Physical System (CPS) application field. This research area is characterized by systems that need to be aware of their own status, the stimuli coming from the environment and the user, which means that they have to be able to sense, react and adapt their behavior accordingly. Specifically, one of the main outcomes of this project relies on the construction of a toolchain to develop systems that use a self-adaptation loop working at runtime in heterogeneous architectures, i.e., a platform where software and hardware resources cooperate [Palumbo'19b. As can be seen in Figure 2-10, this runtime self-adaptation loop is divided into 5 steps:

1. The application is monitored and the environment is sensed.

2. This information is translated to meaningful parameters, also called KPIs.

3. The KPIs are evaluated and compared to predefined requirements.

4. If the requirements are not achieved, the system selects the type of adaptation required to fulfill the requirements.

5. The selected adaptation is carried out, if any.

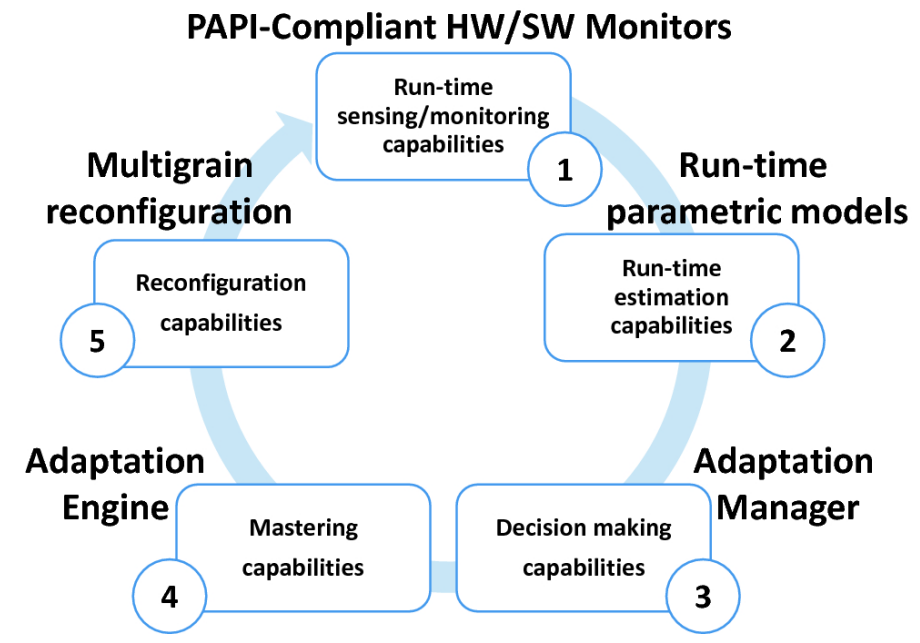

Figure 2-10: CERBERO self-adaptation loop. Image extracted from |Palumbo'19b 


\subsection{Application monitoring}

With the current increment of architecture complexity and its counterpart on the application development field, the performance analysis of applications running on specific platforms is becoming a complex task. Additionally, to fulfill this task, the developer needs to have a deep knowledge of both the architecture and the application. In this section, an overview of the different monitoring tools available in the state of the art is presented. This analysis has been divided considering the Abstraction Level that these tools provide. This aspect is specially relevant in this work because, in this case, the information retrieved through the monitoring infrastructure will be used to profile applications considering non-functional aspects, such as execution time and/or energy consumption.

In order to carry out this performance analysis, two different approaches are addressed in this section: PMC-based monitoring and dataflow analysis. The former analyzes the application based on hardware events like memory utilization, instructions executed and/or clock cycles. In this sense, first, PAPI library, whose main objective is to give access to a set of PMCs gathering this information, is presented and, after that, several monitoring tools based on this library are analyzed. The latter extracts system-level information and profiles the application associating this information to the different tasks composing the application.

\subsubsection{PAPI}

Currently, modern processors gather a set of $\mathrm{PMC} s$, which retrieve performance information at runtime. These PMCs count the occurrences of specific events, e.g., a clock cycle or a data miss when accessing the cache memory, while the application is running on the target platform. By including these counters, manufacturers expect to ease the performance analysis of their platforms and to avoid the use of software traces or similar, which are quite expensive in terms of time overhead.

However, considering the cornucopia of architectures available in the market, e.g., multi-/many-core processors, GPUs, heterogeneous platforms, etc., the retrieval of this information may require a deep understanding of the architecture. In this sense, PAPI library aims at providing a standard way of accessing low-level hardware monitoring information by means of a dedicated API [Terpstra'10]. To tackle this variability, PAPI library has been organized into two layers, as shown in Figure 2-11.

- Low level: to deal with the individualities of each resource, PAPI low level is composed of different components associated to each type of resource. As a result, each component contains the architecture specific routines to access and manage PMCinformation. 
- High level: to unify the access to every component, PAPI high level provides a unique API to access the aforementioned components. The availability of these components is defined by the user when compiling the library.

With this separation of concerns, $\mathrm{PAPI}$ provides a standard and unique way of accessing resources of different nature, e.g., x86 or ARM processors [Haidar'17 $\left.\right|^{2}$.

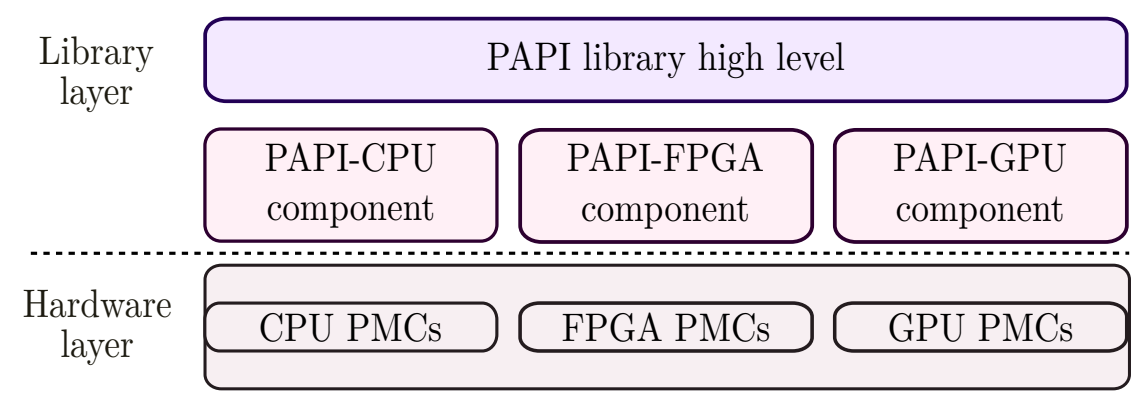

Figure 2-11: PAPI library block diagram

Similarly to the structure to access PMC information, the data that can be retrieved from each resource can also be organized into two different categories:

- Native events: the set of events that are architecture dependent and, hence, are defined within each $\overline{\text { PAPI }}$ component. For example, two native events that could be used to monitor data or instruction L1 cache misses could be $A R C H \_L 1 \_D C M$ and $A R C H \_L 1 \_I C M$, respectively.

- Preset events: the set of predefined events provided by PAPI These events aim at abstracting the definition of events and are architecture independent. For this reason, each component has to define the relationship between their native events and the preset ones. This relationship can be either a direct one or a combination of several native events. Following the previous example, $A R C H \_L 1 \_D C M$ could be directly related to PAPI_L1_DCM preset event, while PAPI_L1_TCM, which represents the total amount of cache misses, could be associated to the addition of both $A R C H \_L 1 \_D C M$ and $A R C H \_L 1 \_I C M$.

\subsubsection{PMC-based monitoring}

Although PAPI itself is the most representative tool in the PMC-based application monitoring area, it is also common to use PAPI as a middle-ware to develop profiling, tracing and sampling toolkits, which main aim is to access PMC information. This is the case of, for example, HPCToolkit, Score-P, Vampir or papiex.

\footnotetext{
${ }^{2}$ Refer to https://bitbucket.org/icl/papi/wiki/Supported-Architectures.md for a list of the supported architectures

${ }^{3}$ Refer to https://icl.cs.utk.edu/projects/papi/wiki/PAPIC:PAPI_presets.3 for a list of preset events
} 
PAPI [Terpstra'10], as explained before, provides an architectural abstraction in the PMC-based monitoring. This tool is composed of a dedicated API that unifies the access to this information. Currently, PAPI developers seek to help in the classification and categorization of the available events in the different architectures, as they are also growing in complexity [Danalis'17]. Additionally, this tool is aiming at increasing its usefulness in $\mathrm{HPC}$ community by extending $\mathrm{PAPI}$ with a new $\mathrm{PAPI}++$ software package and, hence, offering a more efficient and flexible software design ${ }^{4}$. As a drawback, PAPI does not provide specific routines to automatically instrument code or to graphically represent the data, as they are mainly focused on abstracting low level details.

HPCToolkit Adhianto'10 is an integrated tool suite to measure and analyze program performances, which is mainly targeting HPC supercomputers. This tool matches PMC event occurrences retrieved through $\overline{\text { PAPI }}$ to a specific thread/process. To do so, it employs a sampling technique to reduce the monitoring overhead and, as it works in parallel with the real application execution, it does not need to modify the system binaries. However, it requires costly post-processing to correlate event occurrences with specific pieces of the application.

Score-P [Schlütter'14] and Vampir [Knüpfer'08] are two tools that cooperate to 1) profile code and 2) graphically represent performance information, respectively. The former is presented as an instrumentation tool that provides a configurable level of code instrumentation depending on the granularity developers want to have and the overhead they can tolerate. The latter performs PAPI event analysis and represents it in a graphical way. Even if with these tools the monitoring can be extended to Message Passing Interface (MPI) and OpenMP information, the configuration of PAPI events must be homogeneous for the whole application and architecture, limiting, thus, possible heterogeneity that developers may require during the analysis.

papiex [Mucci'07] is an abstraction layer provided by PAPI developers aiming at increasing even more the transparency when profiling applications. In this sense, this tool is executed through command line together with the application to be monitored by pinpointing the events that the user wants to monitor. With this information, the tool is able to configure the monitoring, detect the creation/destruction of threads and dump the monitoring information to a file. However, as in the previous case, the performance monitoring must be homogeneous for the whole application and architecture. Consequently, no heterogeneity is supported.

\footnotetext{
${ }^{4}$ Information extracted from https://www.icl.utk.edu/files/publications/2019/icl-utk-12402019.pdf
} 


\subsubsection{Dataflow analysis}

These tools are focused on the analysis of the application from a system-level point of view. As this approach is based on the dataflow MoC, the analyzability of the applications is enriched and, even at design time, some functional characteristics can be mathematically extracted, e.g., data transfers, data dependencies, etc. In this sense, dataflow profiling tools can either profile applications in design time (Turnus) or, on the contrary, take advantage of the system-level application configuration so as to characterize it in an actor-wise way, as in the case of Parallel Runtime Scheduling and Execution Controller (PaRSEC).

Turnus Casale-Brunet'15, Casale-Brunet'13 is a tool extending the functionalities of ORCC simulator implemented in CAL dataflow language. This tool includes, for example, executed operators counters and instruction counters that make possible the generation of scheduler independent traces including only the dependencies between tasks. To sum up, Turnus performs a profiling during the application design and provides runtime schedulers with enough information to refine their workload distribution. Additionally, this information can be used by the developer to refine their implementations and, hence, optimize the application deployment by, for example, reducing the memory footprint on an FPGA.

PaRSEC [Bosilca'13, Hoque'17] is a generic framework for task-based scheduling and management on distributed HPC heterogeneous architectures. In [McCraw'14, PAPI has been used to perform a power characterization of the application when it is managed by PaRSEC. In this work, it has been demonstrated the usefulness of profiling applications in a task-level, as it provides developers with a clear relationship between application and, in this specific case, power consumption.

\subsection{Energy modeling}

As explained in [Jejurikar'04], the energy consumption of a System-on-Chip (SoC) can be analyzed following the schema of a Complementary Metal Oxide Semiconductor (CMOS) transistor. [Jejurikar'04] proposes a model, graphically represented in Figure 2-12, which divides the power consumption depending on its associated current:

- $P_{\text {stat }}$ : the static power consumption is associated to the transistor leakage $\left(I_{\text {leak }}\right)$, which is always present even if there is no switching activity.

- $P_{d y n}$ : the dynamic power consumption is associated to the transistor switching activity $\left(I_{\text {switch }}\right)$, which is due to the charging/discharging of its capacitance. 
- $P_{\text {short }}$ : the short power consumption is associated to the shortcut current that momentarily appears when the transistor switches $\left(I_{\text {short }}\right)$.

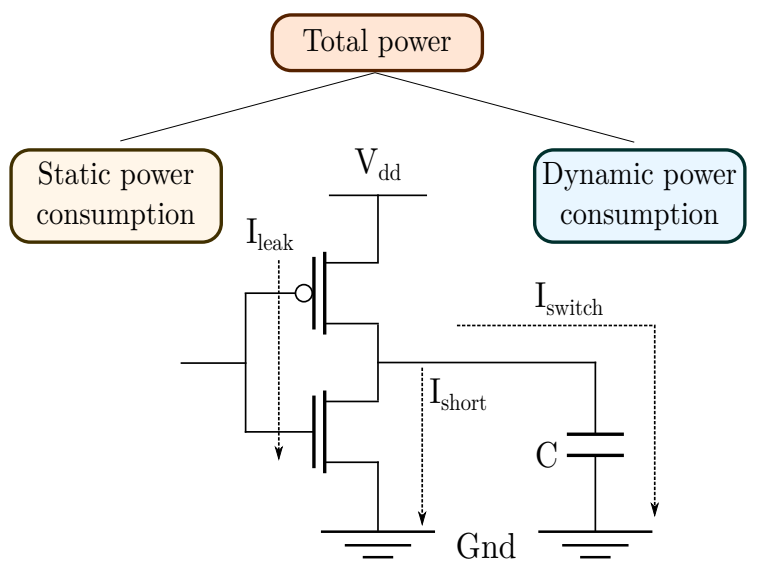

Figure 2-12: Power consumption schema. Based on image from [Nogues'16a]

Although in the past $P_{d y n}$ was the predominant contribution to the system power consumption, currently, the arrival of nano-metric sizes in the transistor technologies has led to an increment in $I_{\text {leak }}$ which, in turn, has increased the relevance of $P_{\text {stat }}$. Consequently, nowadays, the only contribution that can be neglected is the one associated to $P_{\text {short }}$. As a result, as explained in [Jejurikar'04, Nogues'16b|, the power consumption of a system can be represented as shown in Equation 2-1.

$$
P_{\text {tot }}=P_{\text {stat }}+P_{d y n}
$$

Therefore, in order to model the energy that is consumed in a system, both sources need to be considered. In this line, in Subsection 2.5.1, the levels of abstraction that can be considered to carry out this modeling are analyzed. On top of that, different benchmarking techniques used in the literature to analyze the system power response are addressed in Subsection 2.5.2. Finally, the models themselves can be extracted following different mathematical approaches. This last aspect is evaluated in Subsection 2.5.3.

\subsubsection{Model definition}

The first task when modeling an architecture is to define the type of model to be extracted. In this phase, the required level of abstraction has to be selected weighting both accuracy and simplicity. While lower levels of abstraction will have better accuracies, higher levels of abstraction will provide simpler models and, thus, faster estimations. In this section, based on [Brandolese'00] categories, the levels represented in Figure 2-13 are analyzed. 


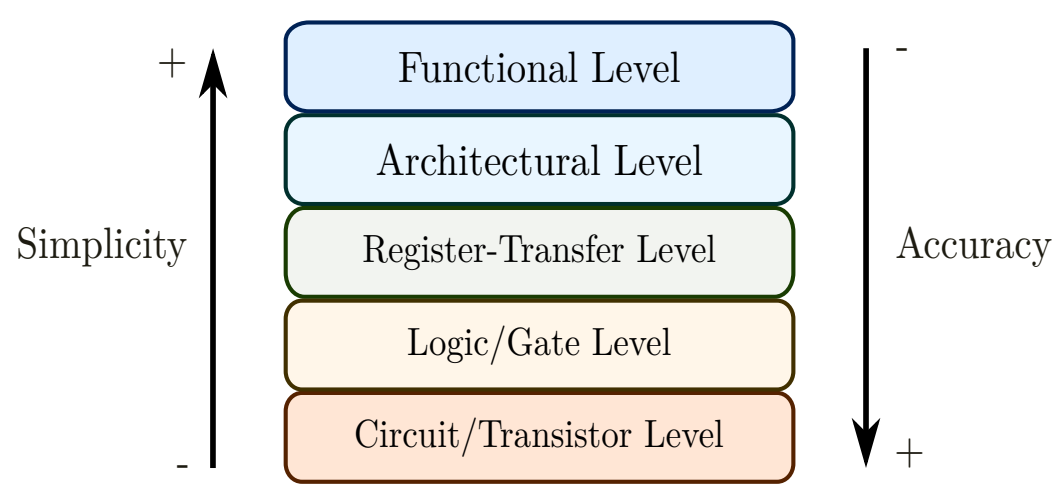

Figure 2-13: Levels of abstraction when modeling architectures

The models based on Circuit/Transistor Level are the ones with the highest accuracy. However, as the system is represented with the set of transistors, the routing and the layout, this type of models becomes extremely complex. Additionally, the development of this type of models usually requires large times and an extreme knowledge of the architecture low-level. In this line, tools estimating system power/energy consumption considering this level of abstraction are based on simulation. For example, PowerMill [Huang'95] is able to simulate current and power consumption of Very-Large-Scale Integration (VLSI) circuits. These simulations are very accurate but, as they are performed in the continuous-time domain, the simulation time is significant. As a result, this type of model is recommended for small circuits, but the modeling of multi-/many-cores architectures is unaffordable.

In order to reduce simulation times, Logic/Gate Level models represent the system as a combination of logic gates, which leads to discrete-time domain. This approach reasonably reduces the system representation complexity with an almost negligible lost of accuracy [Brandolese'00]. Nevertheless, the level of abstraction is still too low and multi-/many-core description is still unfeasible.

The last abstraction layer basing its estimations on simulation is the RegisterTransfer Level. This approach characterizes the architecture as a digital system composed of registers (synchronous D flip-flops) and logical operators (combinational blocks). Each block of the system is characterized in terms of power considering different inputs, as this parameter has a direct impact on its consumption [Bogliolo'00]. Additionally, in [Potlapally'01], this idea is extended to apply different macro models depending on the system inputs and outputs in every simulation cycle. Consequently, the accuracy retrieved by this model is still high but, on the contrary, the simulation time is excessive to be used when estimating complex architectures.

In the case of Architectural Level, the estimation is performed based on information directly retrieved from application executions. This model divides the processor in the real components, i.e., the register file, the instruction caches, the Transaction Look-aside Buffer (TLB), etc. For example, in [Li'98] the Avalanche framework is presented. This tool correlates the platform power consumption with the Instruction Set Simulator (ISS) log trace obtained during the application execution. 
Finally, contrary to the previous approach, in the Functional Level the resources composing the system are classified based on their functionality. A clear example of this classification can be found in [Pelcat'16, Pelcat'17], where a standard MoA is presented. As shown in Figure 2-14, this modeling approach divides the architecture in functional blocks called $\mathrm{PE}$ and communication nodes connecting the processing resources. On top of that, each element represented in the model is associated to a function cost that can be used to estimate, for example, timing or energy consumption. Similarly, Roux'16 presents a modeling technique based on the communications performed within the application under study and it is mainly aimed at performing fast DSE targeting heterogeneous multicore architectures.

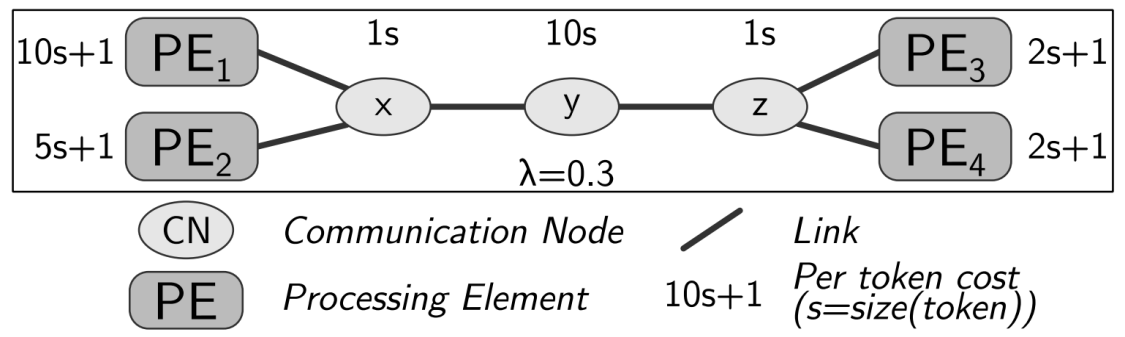

Figure 2-14: MoA example. Image extracted from Pelcat'17

\subsubsection{Benchmarking}

In general, platform modeling is divided into two steps: model development and, in this case, energy estimation. The former basically aims at stimulating the platform resources under a set of predefined constrains so as to measure the behavior of those resources and extract the specific parameters of the model. The latter, on the contrary, is based on analyzing the response of the architecture to the application under study and correlating the response of the platform with the model previously extracted, obtaining, thus, its associated energy estimation.

During the model development stage, the different ways to stimulate the platform are usually known as benchmarking. In this line, depending on the level of abstraction the model is based on, the methodology used to stimulate the architecture also varies.

In the case of Circuit/Transistor and Logic/Gate levels of abstraction, the parameters of the model are extracted by performing a low-level power analysis of the system architecture |Huang'95|. Normally, the information linked to the power or energy consumed by each component is not even available in the system data sheets, so analyzing platforms at these levels is a extremely time-consuming task.

Considering the Register-Transfer level of abstraction, the power measurements are carried out by isolating each combinational block from the rest of the design. Each part of the architecture is independently characterized under a set of defined conditions. For example, in [Potlapally'01], each block has its own set of power modes, which are associated to the consumption obtained for different input scenarios. 
When using the higher levels of abstraction, i.e., Architectural and Functional levels, depending on the information that will be used to build the energy consumption model, the strategy used to stimulate the architecture may differ.

In the case of [Pelcat'17], the energy consumption model will be developed based on MoCinformation, while no runtime information will be required to perform energy estimations. That is, this modeling technique can not only be used to estimate the energy consumption after running the application on the architecture under study, but also to predict its consumption. To do so, the application designed following a specific $\mathrm{MoC}$ is characterized with its communication and computation quanta. The former is used to extract the communication among application tasks. The latter defines the processing required to fulfill each task. In the case of [Pelcat'17], they tested a single application with different levels of parallelism and deployments. Specifically, they use a stereo-matching application and 1,530 different tests so as to extract the cost functions associated to each $\mathrm{PE}$ and communication link. In each test, the application configuration, which modifies both quanta, and its deployment are modified.

Finally, in the case of [Roux'16], a $\mu$ benchmarking technique has been selected to develop their communication-based model. This approach is based on building a set of simple kernels where each of them is specially devoted to stress one part or one specific functionality of the architecture. These kernels follow the structure shown in Algorithm 2-1, where, as explained in [Roux'16], one particular type of operation with specific parameters -size- is iteratively running during a time slot -timeTest-. Furthermore, these kernels can, on the one hand, be focused on stressing the platform communication in different ways, using different channels, different token sizes, etc., or, on the other hand, they can intensively perform different types of computation. With this approach, the platform resources are independently stressed and their average power/energy consumption can be correlated with the model parameters.

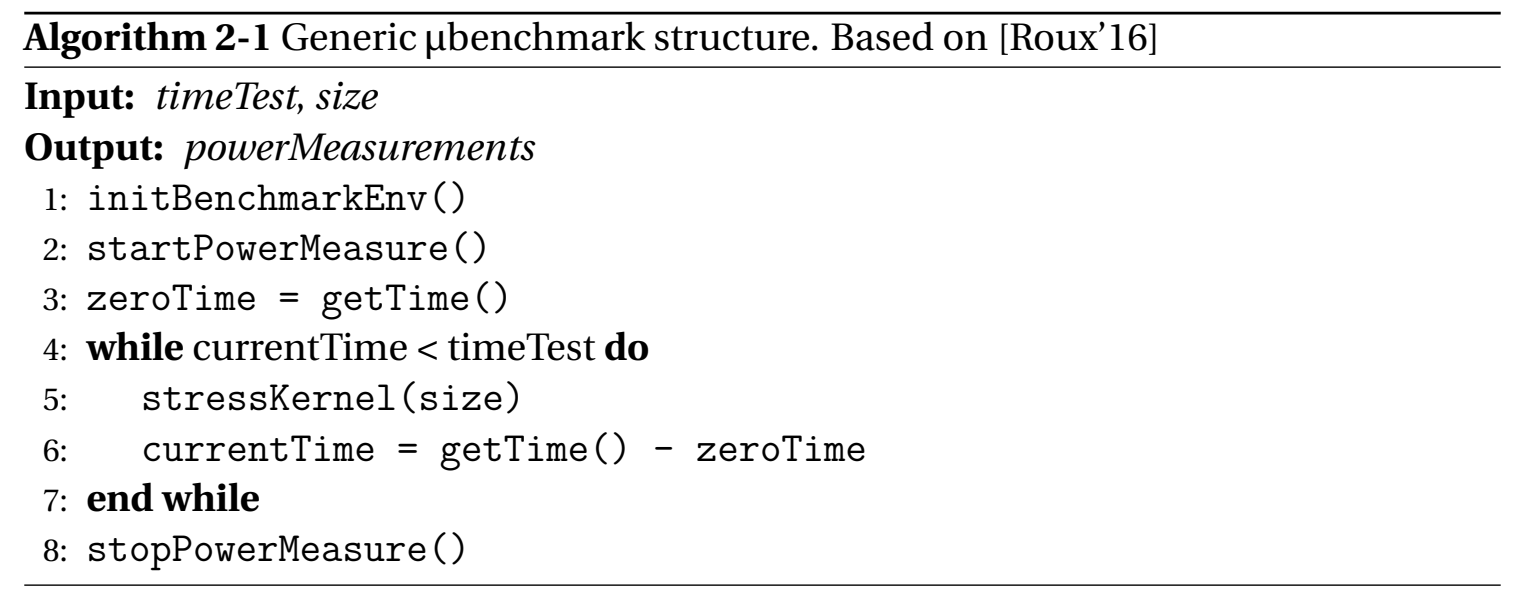




\subsubsection{Model extraction}

Once the state of the art associated to the model definition and the platform benchmarking has been analyzed, this part of the state of the art analysis focuses on the extraction of the actual values of the energy consumption model. Specifically, two different procedures, which correlate both model parameters and energy consumption information, are studied.

First, in the lowest levels of abstraction, the analytical modeling is applied. This approach is based on statistically expressing the power/energy consumption of each component of the system and, after that, combining these partial models in order to have a system-level model [Kamble'97]. The complexity of these models grows with the complexity of the system so, in the case of multi-/many-core architectures, the expressions and, thus, their definition, become extremely complex.

The most common approach is to apply linear regression to adjust the coefficients of the model. This strategy can be applied to either low or high levels of abstraction. For example, in [Bogliolo'00], where a Register Transfer level model is developed, this methodology is applied so as to correlate input and output activity with the power dissipation of each component. In the case of [Pelcat'17], linear regression is employed to associate the MoC quanta application activity with the power consumed by each $\mathrm{PE}$ and communication link. Consequently, linear cost functions are extracted, matching the idea of obtaining a Linear System-Level Architecture (LSLA) model.

As explained in |Nogues'16b|, given a set of polynomial inputs that are composed of the power measurements obtained during the benchmarking, the problem to be solved through linear regression can be expressed as in Equation 2-2. In this equation, $\mathbf{y}$ corresponds to the power measurements, $\mathbf{X}$ is the set of observed parameters, being $\mathbf{X}=\left[x_{1}, x_{2}, \ldots, x_{n}\right], \mathbf{a}$ is the set of coefficients to be adjusted and $\varepsilon$ is the error, that is, the portion of data that is not represented after refining the regression coefficients.

$$
\mathbf{y}=\mathbf{X a}+\varepsilon
$$

This problem can be solved through Minimum Mean Squared Error (MMSE), whose main objective is to adjust a coefficients to obtain the $\mathbf{a}$ that minimizes the minimum squared error, which corresponds to Equation 2-3.

$$
\hat{\mathbf{a}}=\left(\mathbf{X}^{\prime} \mathbf{X}\right)^{-1} \mathbf{X}^{\prime} \mathbf{y}
$$

Therefore, once the model has been extracted and the linear regression has adjusted the coefficients, estimations $\hat{\mathbf{y}}$ that follows Equation 2-4 could be obtained. In this case, the curve that better fits the data trend will be obtained.

$$
\hat{\mathbf{y}}=\mathbf{X a ̂}
$$

Finally, as presented in Kleinbaum'88, Glantz'90, the coefficient of determination $R^{2}$ can be used to represent the percentage of data that is properly represented by $\hat{\mathbf{y}}$. 
This value is computed as expressed in 2-5, where $\overline{\mathbf{y}}$ represents the average value of the measured data $\mathbf{y}$, while $\hat{\mathbf{y}}$ represents the values generated using the extracted model. Consequently, the higher the value of $R^{2}$, the better the model represents the data. That is, $1-R^{2}$ is equivalent to the aforementioned $\varepsilon$, which is the portion of data that is not represented by the model.

$$
R^{2} \equiv 1-\frac{\|\mathbf{y}-\hat{\mathbf{y}}\|^{2}}{\|\mathbf{y}-\overline{\mathbf{y}}\|^{2}}=\frac{\|\hat{\mathbf{y}}-\overline{\mathbf{y}}\|^{2}}{\|\mathbf{y}-\overline{\mathbf{y}}\|^{2}}=\frac{\operatorname{var}(\hat{\mathbf{y}})}{\operatorname{var}(\mathbf{y})}
$$

\subsection{Energy consumption reduction}

As explained in Section 2.2, the development of current chips is following Moore's law, which states that the power density is the main limitation when trying to increase single-chip processing capabilities. In this line, there is a current trend which tries to reduce, as much as possible, the power consumption without loosing performance [Jejurikar'04]. Additionally, in Section 2.5, the power consumption has been divided into two main sources: static $\left(P_{\text {stat }}\right)$ and dynamic $\left(P_{\text {dyn }}\right)$.

A set of techniques to reduce the power associated to the transistor leakage $\left(P_{\text {stat }}\right)$ are explained in Subsection 2.6.1. Subsection 2.6.2 details, in turn, some strategies to reduce the energy consumption associated to the transistor switching activity $\left(P_{d y n}\right)$.

\subsubsection{Static consumption reduction}

The static power consumption $\left(P_{\text {stat }}\right)$, as mentioned before, is the one associated to the transistor leakage, that is, the current that exists even when the transistor is not switching, which is originated due to device imperfections. The relevance of this part in the total power consumption increases together with the reduction of the transistor size technology |Wiltgen'13|. The increment in the number of transistors used in a single-chip and the severity of the imperfections in sub-micrometer sizes are the main reasons behind this trend.

Considering that the static power consumption is intrinsically linked to the technology employed to build the device, the techniques applied to reduce its contribution are limited. The most extended one among the literature is power gating. This technique is based on shutting down some blocks of the system relying on the fact that without power supply, the transistor leakage disappears. This methodology, as shown in Figure 2-15, usually follows the strategy of finishing the workload processing as fast as possible and forcing the system to enter a sleep mode to save power, if the remaining time slot is long enough. 


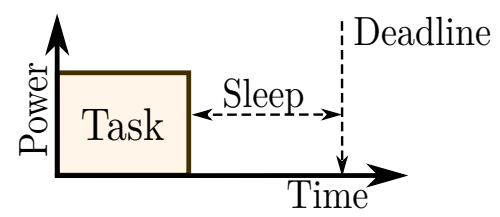

Figure 2-15: Power gating example: fast processing followed by a sleep mode time slot

In [Jiang'05], the costs and benefits of applying a basic power gating at a system level are analyzed. In this study, it has been observed that the reduction of static power comes with a cost in terms of dynamic power and area. In order to reduce the leakage, some sleep transistors are included, which, in turn, consume area and power. As a result, it has been demonstrated that the leakage is reduced, in average, $47 \%$ with an associated cost of $4 \%$ in area and 5\% in dynamic power, which, as they stated, is worth it considering the increasing importance of leakage power contribution.

In order to improve the usability of power gating at runtime, |Agarwal'06| proposes a technique in which several sleep modes are used to balance out leakage saving and wakeup overhead. In this work, the static power consumption is reduced up to a $17 \%$ using two intermediate sleep modes between active and shutdown states. In these intermediate modes the system is not fully shutdown and, even if it is consuming some power, its wakeup latency is also reduced. Consequently, the sleep mode can be selected depending on the idle slot length.

In a real application where both $\mathrm{CPU}$ and GPU coexist and cooperate, this technique can also be applied. In [Arora'15], a linear prediction model is used to estimate the length of idle states in the system. This is specially important in this kind of systems because, if power gating is applied, the cache memory needs to be flushed before applying it and recovered after waking-up, making power gating a very time consuming operation. In [Arora'15], they demonstrate that their technique provides power savings over $8 \%$ without losing any performance.

\subsubsection{Dynamic consumption reduction}

Regarding dynamic power consumption $\left(P_{d y n}\right)$, as aforementioned, it is mainly associated to the activity within the transistors, i.e., the charging/discharging of their capacitance. As a consequence, this means that the higher the system working frequency is, the higher the dynamic power consumption becomes [Shang'02]. In this context, dynamic power reduction techniques focuses on reducing the number of transitions in the circuitry. Some examples of this kind of methodology that can be found among the literature are 1) clock gating, 2) DVFS and 3) task migration.

Clock gating has been widely used by the scientific community to reduce dynamic power consumption [Wu'00, Donno'03]. This technique disables the clock of some blocks in the system to avoid unnecessary transitions in transistors, thus stopping their dynamic consumption. The principle behind this technology is that the clock signals are mainly used to synchronize blocks along the system and, in contrast, they do not 
carry information by themselves. Consequently, when specific parts of the system are not used, their clock can be disconnected [Kathuria'11]. Similarly to the power gating technique, the structure of this strategy is also composed of fast processing slots followed by idle time slots. However, in this case, as represented in Figure 2-16, the power consumption of the idle state is not as small as in the previous case.

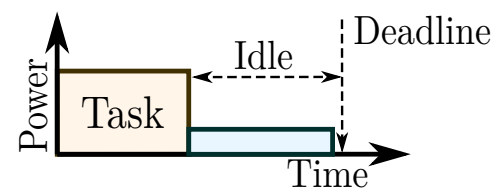

Figure 2-16: Clock gating example: fast processing followed by an idle mode time slot

In the case of DVFS [Macken'90], the voltage and its counterpart frequency are scaled to fulfill all the tasks before a predefined deadline. Contrary to the previous examples, the system frequency is set to a point in which all the tasks are finished as close to the deadline as possible, avoiding idle slots. This strategy is exemplified in Figure 2-17, By doing so, the average power consumption of the system is reduced and the Quality of Service [QoS) of the application is maintained. [Le Sueur'10] carries out a detailed study about the pros and cons of using DVFS. In this work, the authors conclude that, with the current reduction of the technology size and its associated reduction on dynamic power consumption, the effectiveness of DVFS has been drastically reduced on recent platforms, but it is still worthy on portable devices, as further demonstrated in [Carroll'10]. As an example, [Yang'18] presents a work where, using a modern smartphone, a 50\% of the energy consumed by YouTube application is saved when applying DVFS for both video processing and data download.

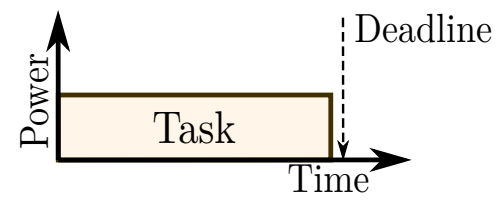

Figure 2-17: DVFS example: frequency is scaled for the task to fill the available time slot

Differently to the previous approaches, task migration does not address the power reduction from the point of view of a single core. In contrast, this technique aims at modifying the application mapping/scheduling to homogenize the chip temperature and/or average the power dissipation of all the resources. For this reason, task migration can only be applied in those systems where multi-/many-cores or heterogeneous architectures are employed. Figure 2-18 shows an example where task migration is used to balance the workload. On the one hand, regarding the usage of task migration to manage the temperature along the system, [Ge'10] presents an implementation on a many-core architecture where all the cores work together to balance the temperature and to reduce the chip hot spots, which can lead to performance detriment and/or power consumption increments [Donald'06]. On the other hand, in [Holmbacka'13], task migration usefulness is shown when the system 
needs to 1) increase the system performance or 2) reduce power consumption. In [Holmbacka'13], the power consumption reduction is obtained by combining task migration with power gating and shutting down idle cores, i.e., the ones with no workload. Similarly, the work presented in March'13 combines task migration with DVFS and obtains energy savings up to $40 \%$, as the workload is balanced and each task is executed using the lowest voltage (and frequency) that fills the available time slot.

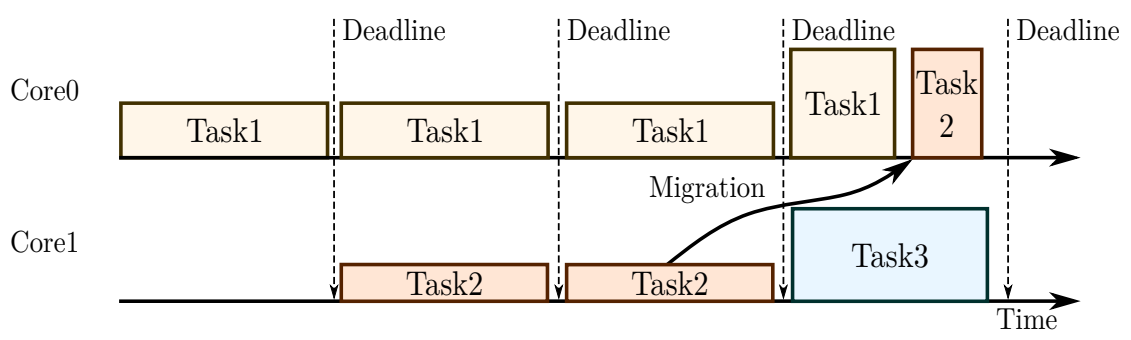

Figure 2-18: Task migration example. Image based on March'13

\subsection{Discussion}

This chapter has thoroughly studied the state of the art related to the different concepts that are required to contextualize the work presented in the rest of this manuscript. Specifically, an independent analysis has been performed for each module composing the Y-chart design methodology where the energy consumption requirement is iteratively optimized, graphically represented in Figure 1-1.

In this regard, Chapter 3 will discuss the different tools and strategies introduced in this chapter so as to build the aforementioned methodology when targeting complex applications running on multi-/many-core architectures. During this evaluation, for each block of the methodology, it will be decided whether an already existing tool/strategy can be employed or a new one should be developed, if a lack in the literature is located. Specifically, the link between both chapters follows Table 2-1.

Table 2-1: Discussion and decisions of each topic presented in the state of the art analysis

\begin{tabular}{|c|c|c|}
\hline Topic being discussed & State of the art & Discussion and decisions \\
\hline HSI application & Section 2.1 & Subsection 3.1.1 \\
\hline HPC & Section 2.2 & Subsection 3.1.2 \\
\hline Design methodology & Section 2.3 & Section 3.2 \\
\hline Application monitoring & Section 2.4 & Subsection 3.2.2 \\
\hline Energy modeling & Section 2.5 & Subsection 3.2.3 \\
\hline Energy consumption reduction & Section 2.6 & Subsection 3.2.4 \\
\hline
\end{tabular}




\section{METHODOLOGY DEFINITION AND TOOLS}

After analyzing the state of the art related to the development of highly demanding applications on complex architectures and its optimization in terms of energy consumption -Section 2.3-, in this chapter, the solution proposed during this $\mathrm{PhD}$ is contextualized. Specifically, both the methodology proposed and the different modules composing its associated toolchain are discussed and defined.

In this line, the chapter is divided into 4 parts. First, the use-case that motivated the development of this methodology is explained in Section 3.1. After that, in Section 3.2, the proposed Y-chart dataflow design methodology is specified and the parts composing the energy-aware feedback loop are presented. Then, in Section 3.3. the tools and materials used to develop a toolchain prototype are exposed. This section focuses on explaining the already existing tools that have been employed as a baseline to develop the toolchain. As a conclusion, a brief discussion is provided in Section 3.4.

\subsection{Use-case}

At the beginning of this $\mathrm{PhD}$ work, in the context of HELICoiD European project, the implementation of an HSI application discerning between healthy and tumorous brain tissues in real-time was faced. To develop the system, an MPPA architecture was selected due to its low energy consumption. As will be detailed in Section 7.2, this first implementation was carried out by hand and both application and architecture required a deep understanding on the development side. In this implementation, only the supervised learning block of the HELICoiD|HSI processing chain was included, as it is the most computationally intensive one.

Once the implementation was finished, the necessity of a methodology that helps in the development of highly demanding applications running on complex architectures was obvious. The rationale behind this was that, as no automatism was included within the development procedure, parallelism opportunities together with more efficient workload distributions may have been missed.

After this, the same processing chain was applied in the dermatological field. However, in this context, energy consumption gains importance, as the system proposed is a hand-held device powered by a battery. Consequently, to include energy- 
awareness, an automatic development procedure became essential, as balancing both performance and energy consumption in the same implementation without any CAD tool is beyond the limits of what a non-expert developer can achieve.

Hence, the use-case that motivates the development of the design methodology proposed in this $\mathrm{PhD}$ and its associated toolchain can be divided into two parts. On the one hand, the HELICoiD HSI processing chain targeting both brain and skin human cancer detection, which will be detailed in Subsection 3.1.1. On the other hand, the MPPA architecture that is used to accelerate the application, whose most relevant characteristics are explained in Subsection 3.1.2.

\subsubsection{Application}

In this use-case, the main objective of the application is to discern between healthy and tumorous tissues using HSI technology. As mentioned before, on the one hand, the processing chain can be applied to an environment without energy constraints such as an operating theater, where brain cancer is distinguished from healthy tissues. On the other hand, HSI can be applied in a hand-held system to detect skin cancer in an early stage, which is crucial in this type of diseases, as explained in Subsection 2.1.4. In this case, energy consumption has to be considered when developing the system, as energy is supplied by a battery and, thus, it is a limited resource.

As introduced in Subsection 2.1.6. HELICoiD processing chain is divided into 4 blocks (Figure 2-2): preprocessing, supervised learning classification, unsupervised learning classification and hybrid technique. As mentioned before, the block requiring acceleration, as explained in [Fabelo'18b], is the supervised learning, which is in charge of classifying the hyperspectral image associating each pixel composing the scene with a specific label and which is going to be described in detail. The supervised learning block is, in turn, composed of 3 algorithms, as shown in Figure 3-2. As can be seen, the input of the block is an already preprocessed hyperspectral image, which is analyzed, in parallel, by both $\mathrm{PCA}$ and SVM generating a one-band representation of the image and a preliminary classification of the image, respectively. After this, both partial results are combined through a K-Nearest Neighbors (KNN) filtering algorithm so as to obtain a refined supervised classification map.

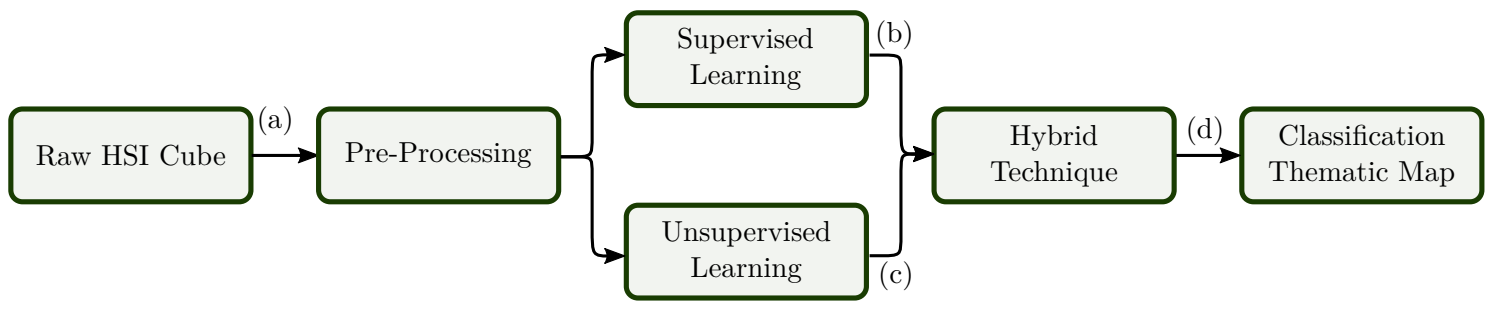

Figure 2-2 3 HELICoiD processing chain (repeated from page 12 


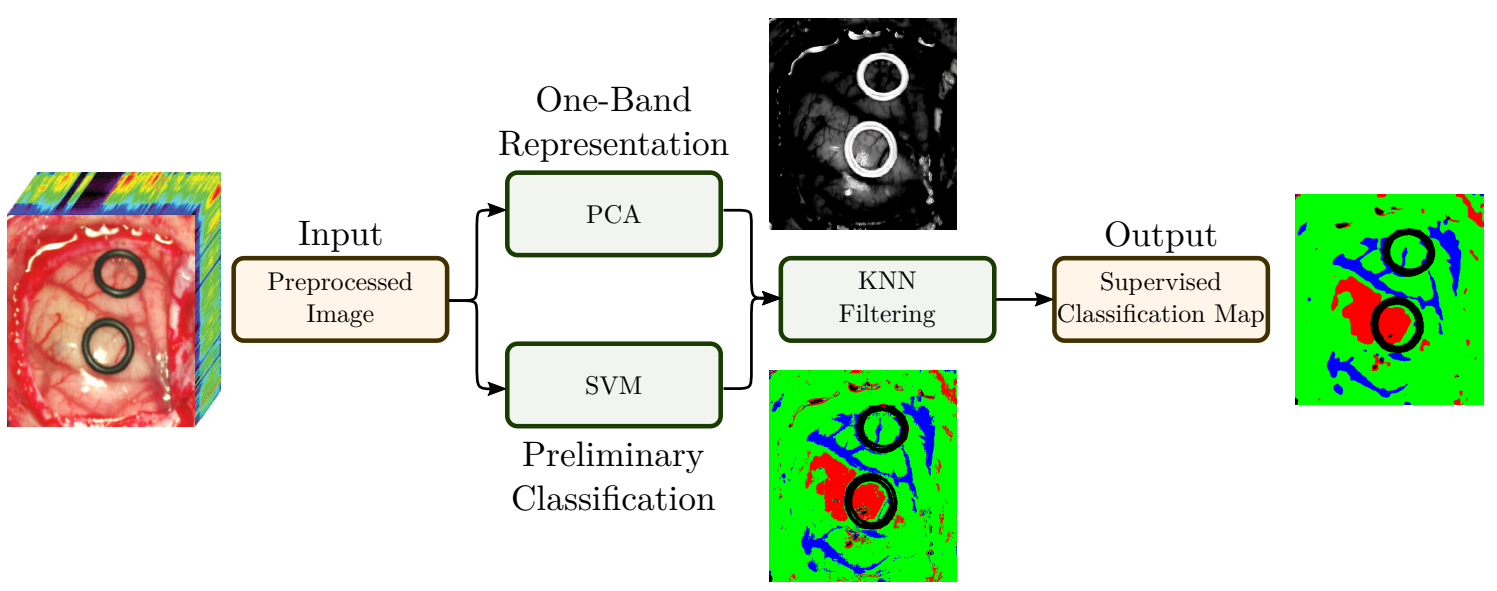

Figure 3-2: HELICoiD processing chain. Supervised classification step

The main objective of PCA algorithm is to simplify the representation of the hyperspectral image. This process is especially important, as HSI technology collects almost continuous information from across the electromagnetic spectrum and, thus, the information between adjacent bands is deeply correlated. Thanks to PCA the redundancies can be removed and, consequently, the amount of data to be processed is drastically reduced without loosing relevant information. In the context of HELICoiD processing chain, this algorithm is used to obtain a one-band representation of the image, so the hyperspectral cube is projected onto the first eigenvector, i.e., the one containing most of the spectral information.

To obtain this result, PCA is composed of 4 steps [Lazcano'17c|: 1) image centering, 2) covariance, 3) diagonalization and 4) projection. First, the average value of each band within the hyperspectral image is computed and removed. Secondly, the covariance of the hyperspectral image is computed by multiplying the image by its transpose. After this, the eigenvectors are extracted using the Jacobi method [Chen'06], where successive planar rotations are applied until all the off-diagonal elements are zeroed. Finally, the image is projected onto the first eigenvector so as to obtain the one-band representation of the hyperspectral image.

In the case of SVM, the main objective is to match each pixel contained in the captured scene with a specific material or class. Specifically, in this case, the probability of each pixel to belong to each class is computed. To do so, the algorithm requires a preliminary step, called training, where a model is built considering a set of samples whose classes have been defined previously by an expert -in this case, a pathologist-. This model, as detailed in [Madroñal'17b], consists of a set of binary hyperplanes separating pairs of classes, each hyperplane being defined as the one maximizing the distance between both classes. Once the model is built, it is used to classify the pixels composing the scene, whose associated class is unknown.

As the training step is done off-line, the part included in the HELICoiD processing chain is the classification. This stage can be applied following a pixel-wise strategy, as only the spectral information of the pixel is used. During the classification procedure, 
first, the distance of the pixel to each binary hyperplane and its associated binary probabilities are computed. These binary probabilities represent the possibility of the pixel to belong to one of the two classes the binary hyperplane separates. After this, all the binary probabilities are combined following a minimization approach and the final probabilities are obtained. These probabilities, which represent the possibility of the pixel to belong to all the classes under study, are the output feeding the KNN filter in the HELICoiD processing chain. However, if SVM is used in a standalone mode, a final stage where the class with the highest probability is associated to the pixel under study is included. This last step has been included so as to represent the preliminary classification shown in Figure 3-2.

Finally, KNN filtering is used to refine the pixel-wise classification provided by SVM. This filtering consists on averaging the probabilities of the SVM classification considering non-local neighborhoods, which are composed of the K nearest neighbors. This distance is computed considering both spectral information -obtained with PCAand spatial information -pixel position in the scene-. By including KNN filtering within the supervised classification block, spatial information is considered, so, as a result, a spatial-spectral classification is performed.

KNN filtering is divided into two steps. First, the $\mathrm{K}$ nearest neighbors are computed following Equation 3-1, where, as mentioned before, both spatial and spectral information are taken into account. In this equation, the distance of the pixel found in row $r$ and column $c$ of the image $\left(p_{r c}\right)$ to the one in row $i$ and column $j\left(p_{i j}\right)$ is computed using the squared 2-norm, computing the spectral contribution $\left(P C A_{r c}\right.$ and $\left.P C A_{i j}\right)$ and the spatial one $(r, i, c$ and $j)$ independently. Likewise, a factor $\lambda$ is considered so as to balance both contributions.

$$
d\left(p_{r c}, p_{i j}\right)=\left(P C A_{r c}-P C A_{i j}\right)^{2}+\lambda *\left((r-i)^{2}+(c-j)^{2}\right)
$$

Once the distances are computed, the $K$ closest pixels are considered the neighbors of $p_{r c}$. Then, the pixel probabilities provided by SVM $\left(P_{i j}\right)$, are filtered averaging the values obtained for the pixel and its K nearest neighbors, which are included within the pixel set called $N$, following Equation 3-2, As a result, a new set of filtered probabilities $\left(O_{r c}\right)$ is obtained for each class and each pixel and, after this, the pixel is associated to the class with the highest probability. As a result, the supervised classification map represented in Figure 3-2 is obtained.

$$
O_{r c}=\frac{\sum P_{i j}}{K}, \forall i j \in N
$$




\subsubsection{Architecture}

As mentioned before, the target architecture of the use-case is an MPPA-256-N platform developed by Kalray. This platform, as explained in Section 2.2, belongs to the many-core family of architectures. The MPPA is characterized by its low energy consumption and the parallelism opportunities offered by its 256 PEs. As a reminder from Subsection 2.2.2, the MPPA is composed of 2 IOs and 16 CCs. Additionally, each CC comprises a total amount of $2 \mathrm{MB}$ of shared memory and 16 cores or $\mathrm{PE}$. On the $\mathrm{IO}$ side, it has $4 \mathrm{PE} s$ and $4 \mathrm{~GB}$ of memory. On top of that, to manage communications among resources, the MPPA platform has a NoC where all the resources are interconnected. To use this NoC, each CC has one DMA thus, the IO presents 4 , since it contains $4 \mathrm{CC}$. This structure is presented in Figure 2-6.

However, concerning the use-case implementation, one of the most important points is the Multiple Instruction Multiple Data (MIMD) structure implemented by the platform. Thanks to this characteristic, each PE can execute 1) different instructions and 2) use different data. The former means that each PE can execute a different task or a different part of the application. The latter allows each $\mathrm{PE}$ to work with different data. With the combination of both, the platform can be programmed so as to process different parts of the application in parallel or, on the contrary, parallelize the same task among different $\mathrm{PE} /$ and let each $\mathrm{PE}$ work with different data.

Finally, to ease the application implementation in this platform, and to efficiently communicate data through the NoC, Kalray provides a dedicated library to perform asynchronous communications [Hascoet'18]. With this mechanism, the communications are controlled from the CC 3 by means of a read/ write protocol that transparently performs the data transmissions through get/ put operations, respectively. Specifically, put operation directly sends the data from the source and writes on the destination memory. On the contrary, get is implemented as a reverse put, which implies, first, a notification to request the transmission followed by the actual transmission.

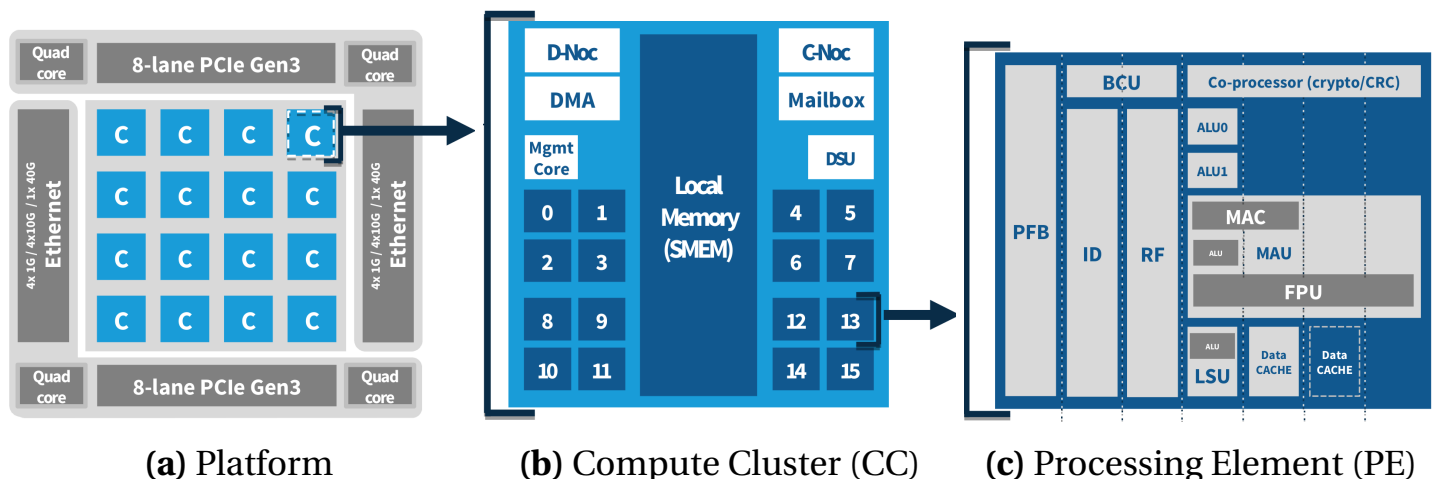

Figure 2-6: MPPA-256-N Bostan structure (repeated from page 15 


\subsection{Application design methodology specification}

As observed during Section 2.3, current application design frameworks aim at providing developers with system implementations where several requirements are considered, thus enabling multi-objective design. Furthermore, considering the current trend where both application and architecture complexity are gradually increasing, different MoCs and MoAs have appeared, respectively, to simplify their representation and to automate most of the application deployment process.

To simplify multi-objective design methodologies where both applications and architectures are represented using models, Y-chart design frameworks are suitable candidates. With this approach, the combination of application specification and architecture definition is performed under a set of user-defined constraints and following a predefined procedure. When this methodology is implemented using dataflow models, DSE can be carried out to optimize one (or more) parameter(s), e.g., latency, throughput and/or energy. However, the obtained implementation might not be optimal when only design time information is employed. For this reason, application profiling can be used to characterize it with real performance information. By doing so, the application design can be refined either at design time, enhancing the user-defined constraints, or at runtime, providing a resource manager with enough information to make decisions targeting the system optimization. As a result, as shown in Figure 3-4, an iterative Y-chart dataflow-based design methodology is proposed in this $\mathrm{PhD}$ to design multi-objective applications based on models.

As can be seen, the iterative nature of the proposed methodology is composed of 4 blocks. First, an application deployment (Subsection 3.2.1) is obtained with a design framework where developers define both the application and the architecture using a Y-chart approach. Secondly, a monitoring block Subsection 3.2.2 is used to profile the application execution. After this, the KPI estimation step (Subsection 3.2.3) is in charge of estimating one (or more) significant indicator(s) based on the profiling data, the so-called KPI(s). Finally, the loop is closed by means of a multi-objective decision making block (Subsection 3.2.4).

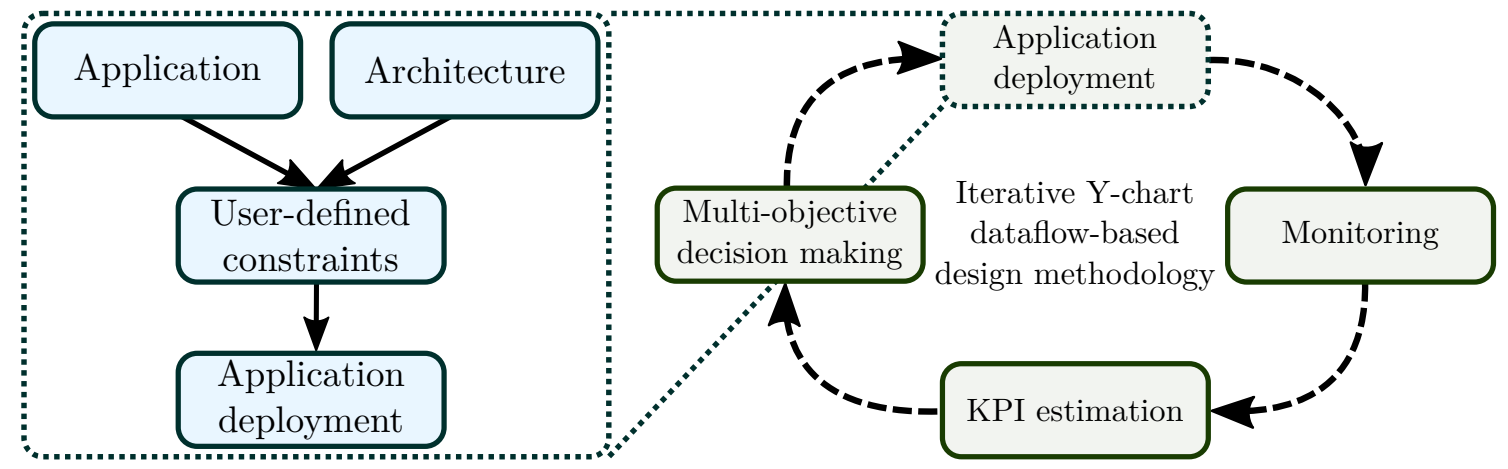

Figure 3-4: Iterative Y-chart dataflow-based design methodology 
As mentioned before, this approach can be applied either at design time or at runtime. In the former, once the application is characterized considering the KPI information, a new application implementation can be generated using DSE with enhanced user-defined constraints. In the latter, at runtime, the resource manager is self-aware of the current system status and, thus, it would be possible to make decisions based on this profiling information.

\subsubsection{Application deployment}

Regarding the application deployment block, it has been decided to use PREESM design framework, based on the 3 main reasons gathered in Table 3-1. First, as explained in Subsection 2.3.3, contrary to SynDEx, PREESM is an open-source Y-chart dataflow-based prototyping tool, which means that, if necessary, its source code can be directly modified. Secondly, PREESM application MoC (PiSDF) has a high level of analyzability, which is not the case of ORCC one (DPN). This feature makes possible the DSE when generating application implementations and, thus, the inclusion of the multi-objective design when generating the code. Finally, as an extra feature, it has a runtime resource manager counterpart: SPiDER. With this extension, selecting this framework provides opportunities to test the proposed iterative design methodology in both design time and runtime. These two tools are detailed in Section 3.3 so as to have a clear understanding of how they work before any modification is included.

Table 3-1: Application deployment design framework comparative

\begin{tabular}{|c|c|c|c|}
\hline Framework & Open-source & Application analyzability & Runtime support \\
\hline PREESM & Yes & High & Yes (SPiDER counterpart) \\
\hline SynDEx & No & High & No \\
\hline ORCC & Yes & Low & Yes \\
\hline
\end{tabular}

Once the framework has been selected, the implementation of the feedback loop needs to be considered. As PREESM and SPiDER are open-source tools, even if the loops are not present, they can be modified to include the missing blocks in order to have a prototype. To that end, the following blocks need to be added: 1) a monitoring infrastructure, 2) a KPI estimation block -which, in this $\mathrm{PhD}$, will be energy consumption- and 3) a multi-objective refinement strategy.

\subsubsection{Monitoring}

Concerning the monitoring infrastructure, the main objective is to retrieve profiling data from real executions; additionally, to tackle the current increment in complexity of both applications and architectures, this infrastructure should support the monitoring of heterogeneous architectures and adapt the monitoring according to the application status. That is, if the application behavior and/or its distribution changes at runtime, 
the monitoring infrastructure should track these changes and adapt itself to retrieve the correct monitoring data. Considering these constraints, it has been decided to follow a dataflow approach where versatile monitoring is considered: a different configuration is supported depending on the actor that is being monitored and the PE (the architecture resource) that is executing it. Finally, since the profiling data is also dependent on the nature of each PE, it will be retrieved using PAPI library-introduced in Subsection 2.4.1-, as it gives transparent access to low-level hardware information.

Once the infrastructure requirements have been settled, the available monitoring options are analyzed. First, although PAPI library itself has not been developed targeting heterogeneous monitoring and the direct management of this library in this context would be too complex, it should be considered that PAPI can be used as a middle-ware to access PMC information. In turn, even though there are plenty of tools built on top of PAPI none of them targets dataflow applications supporting heterogeneous architectures. As a result, it has been decided to build a new monitoring infrastructure from scratch to keep it light-weight. This new tool, one instrumental contribution of this PhD called PAPIFY, will link PAPI-based monitoring configurations to each actor and, at runtime, will select the correct configuration considering the nature of the $\mathrm{PE}$ (the architecture resource) executing the actor. Consequently, PAPIFY, as detailed in Chapter 4 , will be a PAPI-based monitoring infrastructure for Y-chart dataflow-based applications supporting heterogeneous architectures and applications whose functionality and deployment can be modified at runtime.

\subsubsection{KPI estimation}

In the case of the KPI estimation block, two parts needs to be defined: 1) what is going to be estimated and 2) how to estimate it. In the literature, performance and energy consumption are the predominant indicators to be optimized when considering streaming applications, that is, those that run infinitely and execute iteratively.

On the performance side, understood as executions of the iterative application per second -e.g., frames processed per second in a video sequence-, it can be directly measured by timing the execution of each iteration $T_{\text {exec }}$ and applying Equation 3-3.

$$
\text { Performance }\left[\frac{\text { Executions }}{\mathrm{s}}\right]=\frac{1}{T_{\text {exec }}}
$$

In the case of energy consumption, its measurement is not so straightforward, as specific sensors devoted to measure the power consumption of the architecture are required. To deal with this aspect, in this $\mathrm{PhD}$, a new energy consumption modeling methodology is proposed. The novelty behind the proposed approach is that all the information required to build the model and perform the estimations will be available at runtime and, additionally, the model will balance both complexity and accuracy so as to be able to use it at both design time and runtime. Finally, the development of the model will be based on $\mu$ benchmarking, with the objective 
of extracting an application-agnostic energy consumption model of the architecture under study. Specifically, as will be explained in Section 5.1, the architecture model will be composed of 3 independent contributions:

- Resource-active: energy consumed due to the $\mathrm{PE} \beta$ of the target architecture being active. This part will be based on the resource being active or not.

- Communication: energy consumed due to transmitting data among $\mathrm{PE} \beta$ when executing the application. This part will be based on type of memory being used and the data token being transmitted.

- Computation: energy consumed due to the execution of one task on one specific $\mathrm{PE}$. This part will be based on the generated PAPI events.

\subsubsection{Multi-objective decision making}

Finally, the last block to be defined is the one related to multi-objective decision making. As mentioned before, the multi-objective implementation will target optimizing the energy consumption of the application while reaching a user-defined objective of performance (application iterations executed per second). In this sense, as explained in Section 2.6, several techniques aiming at reducing energy consumption already exist among the literature. However, some techniques cannot be always applied as they are either architecture dependent and/or developers need to explicitly apply them. For example, DVFS can only be applied in those architecture where the frequency and/or the voltage can be modified during the application execution. Additionally, there are platforms that automatically apply some techniques to reduce energy consumption. This is the case of the MPPA, where clock gating is applied until one CC is explicitly spawned and its associated clock is enabled.

Nevertheless, in this $\mathrm{PhD}$, the proposal is to automatically reduce the energy consumption considering 1) the techniques that are automatically applied, as in the case of clock gating on the MPPA, where minimizing the spawned CCs will minimize the energy consumption, and 2) employing task migration and energy-aware application mapping/scheduling. In order to implement the last one, two options were considered: to implement a multi-objective mapping/scheduling algorithm that explores the application design space or to tune already existing latencybased algorithms to obtain a multi-objective one. This tuning concept implies to iteratively modify the input data (architecture definition) of already existing latencybased algorithms, which are considered black boxes, so as to obtain different outputs (application deployments). When comparing these two options, it has been considered that the former would provide better multi-objective implementations when using the selected design framework, while the latter would help the community to enhance their design frameworks following the idea of tuning the latency-based mapping/scheduling algorithms that already exist in most of them. In this situation, the second option has been chosen and this energy-aware tuning of latency-based mapping/scheduling algorithms will be described in Section 6.2. 


\subsubsection{Summary of the methodology}

As introduced at the beginning of this section, this $\mathrm{PhD}$ proposes an iterative Y-chart dataflow-based design methodology. Likewise, since the energy consumption is the KPI that will be optimized in this $\mathrm{PhD}$, the methodology has been particularized as an energy-aware optimization loop. Specifically, 4 blocks will be defined along the manuscript to build the 2 proposed versions of the energy-aware optimization loop: one working at design time (PREESM - Section 6.3) and the other optimizing the system at runtime (SPiDER-Section 6.4):

- Application deployment: already existing design frameworks will be used to build the energy-aware optimization loops. PREESM will be used to deploy the application considering design time energy-awareness, while SPiDER will be employed to perform on-the-fly system optimizations.

- Monitoring: a Y-chart dataflow-based monitoring infrastructure called PAPIFY will be introduced in Chapter 4.

- KPI estimation: an energy consumption modeling strategy based on runtime hardware performance utilization will be explained in Chapter 5 .

- Multi-objective decision making: to optimize the system energy consumption, an energy-aware tuning algorithm will be built as a searching mechanism surrounding latency-based mapping/scheduling algorithms. This block will be explained in Section 6.2.

As may have been observed, this iterative design methodology is inspired by the CERBERO adaptation loop explained in Subsection 2.3.4. This is due to the fact that some of the contributions proposed during this $\mathrm{PhD}$ have been developed in the context of this European project.

\subsection{Tools involved}

In this section, the design frameworks that will be used to build the proposed Y-chart dataflow design methodology are detailed. Since these tools already existed before the development of this $\mathrm{PhD}$ work, the objective is to have a clear understanding of how they worked before any modification is introduced. First, in Subsection 3.3.1. PREESM Y-chart design framework is explained. Then, in Subsection 3.3.2, SPiDER, its associated runtime resource manager, is presented. 


\subsubsection{PREESM}

As introduced in Section 2.3, PREESM is a rapid prototyping Y-chart dataflow-based design framework developed at Institut National des Sciences Appliquées (INSA), in Rennes (France). This tool provides developers with both system simulation and a compilable code in a language supported by the target architecture. It needs 3 input files: 1) an application graph, 2) an architecture model and 3) a scenario file.

Regarding the application graph, PREESM uses an extension of SDF MoC called PiSDF. In this regard, both SDF and PiSDF represent the application as a set of nodes (actors) performing data independent operations and communicating through a set of edges (FIFOs). The aforementioned extension consists on including interfaces and parameters within the characteristics of SDF MoC. The former leads to a modular application design in which an actor could be composed, in turn, of a whole nested graph. The latter aims at easily modifying the consumption and production rates of the actors. By doing so, the application can be easily adapted to several scenarios without modifying the inner code of the actors. One example of application graph is shown in Figure 3-5. As can be seen, there are 6 actors communicating through FIFOs and, in this case, there are a total of 9 parameters configuring the application. Precisely, 3 parameters -highlighted in red in the figure- are used to change the application behavior (nbSlices, height and width). Specifically, this application is a Sobel-morpho [Georgakarakos'17] image filter and 1) nbSlices parameter is used to define the parallelism of the application and 2) height and width are used to define the image resolution.

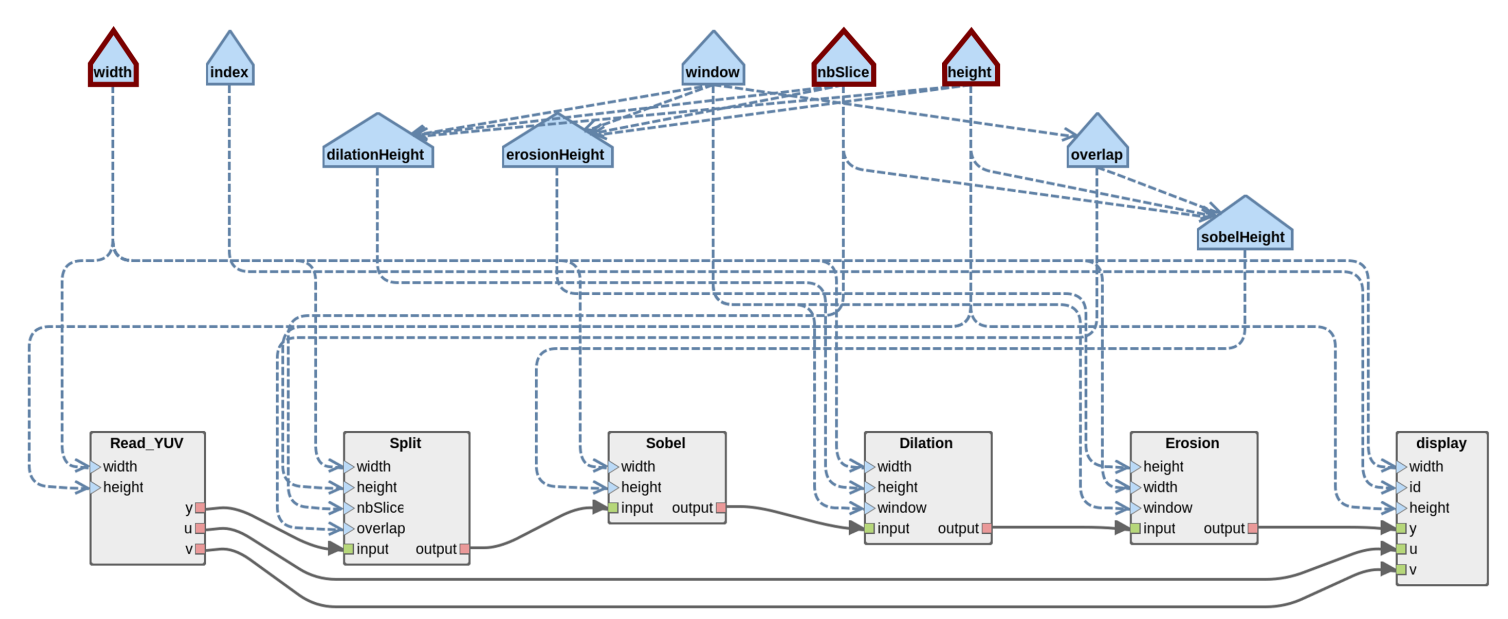

Figure 3-5: PREESM application graph. Sobel-morpho example

Secondly, concerning the architecture definition, PREESM uses S-LAM. With this approach, the architecture is defined as a set of $\mathrm{PE} s$ connected through communication nodes and links. With this model, the dataflow application actors can be linked to specific $\mathrm{PEs}$ that transmit data among them using the FIFOs allocated in the communication nodes. In Figure 3-6, a 4-core architecture where the communication is performed through a shared memory block is shown. 


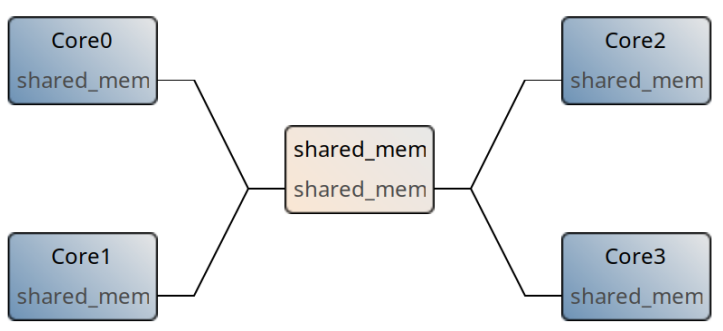

Figure 3-6: PREESM architecture S-LAM graph. 4-core example

Finally, the scenario file is used to include user-defined constraints so as to refine the process of implementing the final system. This file is composed of a total amount of 6 tabs, but 3 of them should be highlighted: Constraints, Timings and Simulation. As an example, Constraints tab is displayed in Figure 3-7.

First, using Constraints users can define specific requirements: for example, in here an actor can be defined to be executed exclusively on a specific. Secondly, in Timings tab the time required to execute each actor on each type of PE can be defined. With this information, PREESM is able to simulate better the different deployments and perform a mapping/scheduling considering heterogeneous architectures. Finally, the Simulation tab gathers the variables that configure the simulation used by PREESM to deploy the application on the architecture. For example, in this tab, the PEs that are in charge of executing special actors that PREESM provides to simplify application definitions can be defined. These special actors cover broadcast/explode/implode actions when distributing information among several $\mathrm{PE}$. Additionally, the different data types used in the application can also be defined, so as to compute the memory transmission sizes and the required memory in each $\mathrm{PE}$.

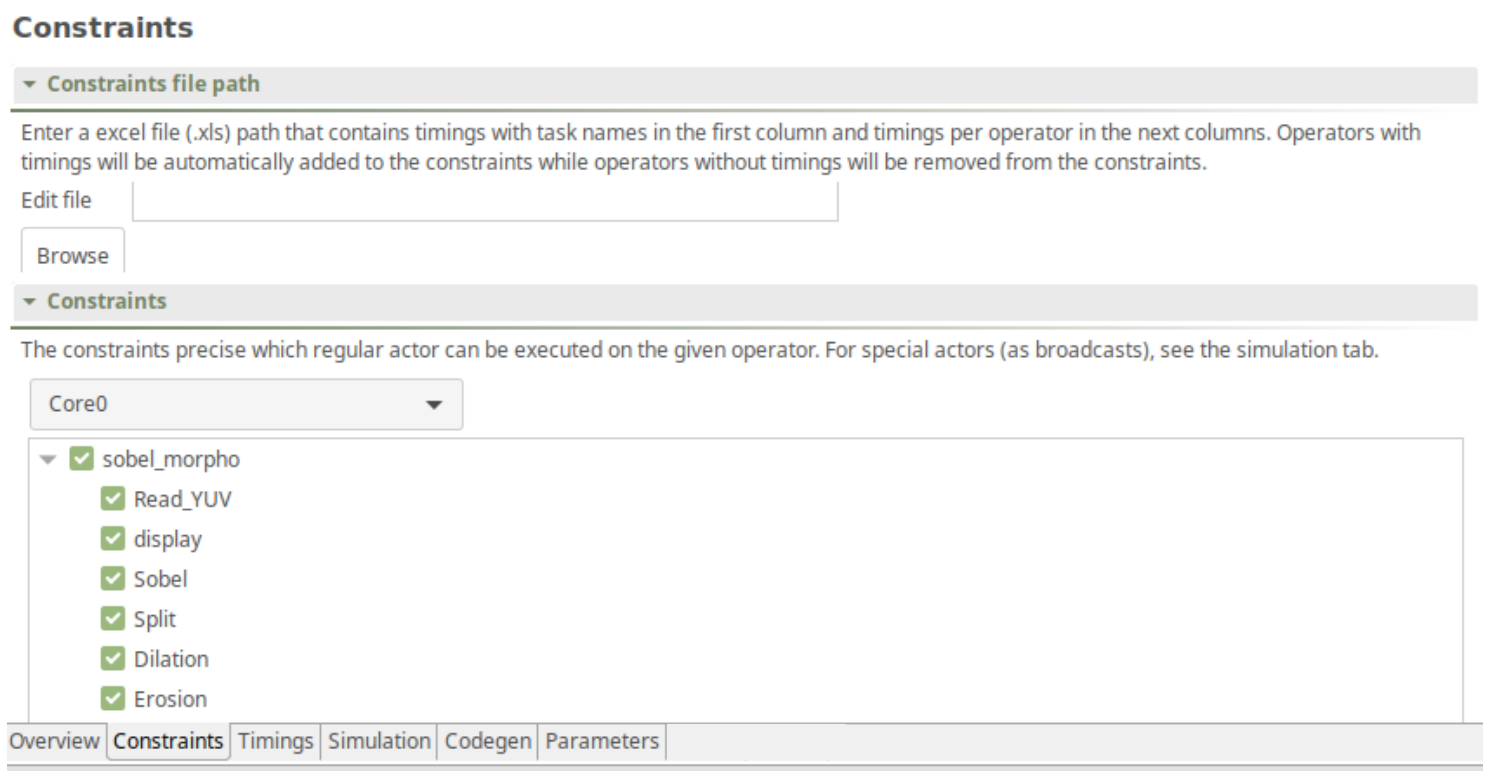

Figure 3-7: PREESM scenario. Constraints example 
Once these 3 files are provided, PREESM uses a file called workflow to generate the final deployment. One example of workflow is shown in Figure 3-8, In this figure, 4 tasks have been highlighted in red: PiMM2SrDAG, PiSDF Scheduling, Memory Allocation and Code Generation.

1. PiMM2SrDAG task flattens the PiSDF application specification in such a way that a Single-Rate DAG (SRDAG) is obtained. Thanks to this step, the parallelism of the application is explicitly exposed and, thus, each actor is replicated as many times as firings will happen in a single iteration of the whole graph. To graphically represent this transformation, Figure 3-9 shows the SRDAG associated to the application shown in Figure 3-5 with a parallelism of 4 slices.

2. Scheduling task is in charge of performing the mapping/scheduling of the dataflow application on the available resources of the architecture. During this procedure, only latency-based algorithms can be selected, whose main objective is to provide an implementation that minimizes the latency of the application.

3. Memory Allocation task reserves the memory that will be used on each PE to store all the input/output data that will be used during the system execution.

4. Code Generation task is in charge of analyzing both the deployment proposed by the Scheduling task and the memory allocated in the Memory Allocation step and generates compilable code for the target architecture. This task is the only architecture-dependent one and, thus, applications developed in PREESM can be easily ported from one architecture to another by changing the backend of the generated code. In this line, the supported architectures are: x86 and ARM multi-cores, Texas Instruments Keystone I \& II Digital Signal Processings (DSP), Xilinx Zync SoC and Kalray MPPA(Bostan version) many-core.

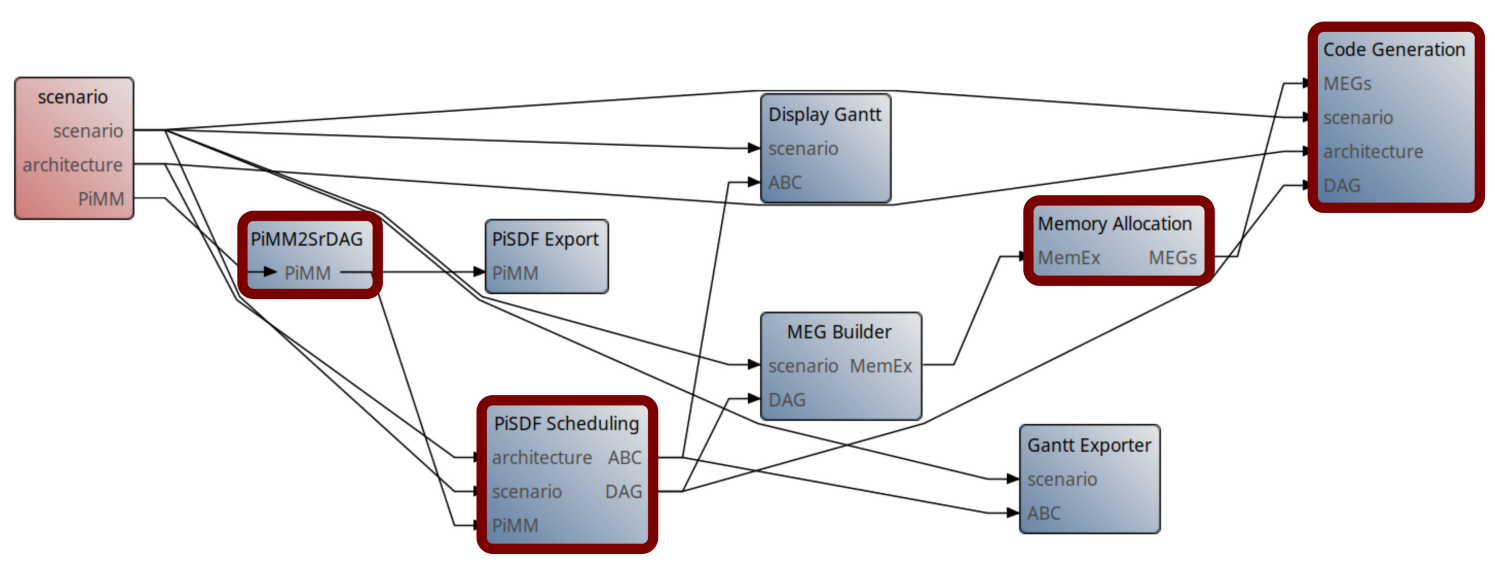

Figure 3-8: PREESM workflow 


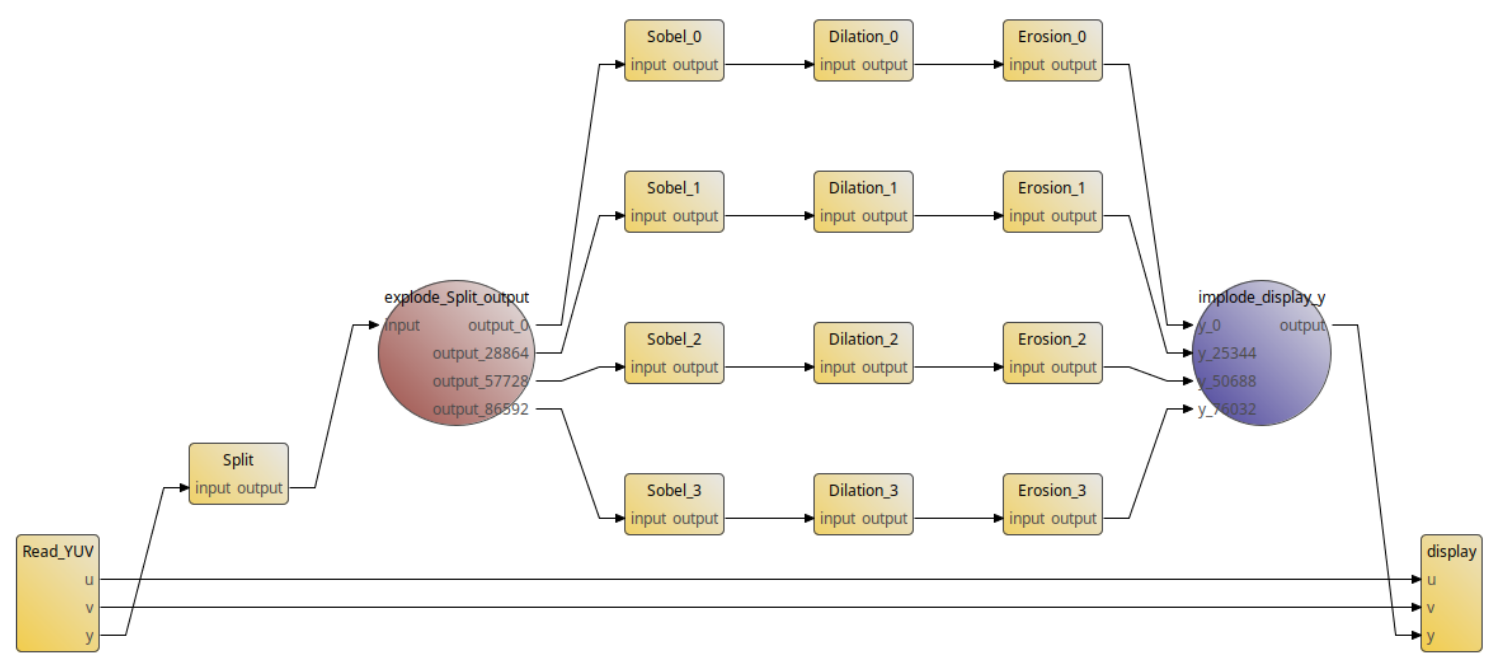

Figure 3-9: SRDAG of Sobel-morpho example with a parallelism degree of 4

\subsubsection{SPiDER}

In order to implement the runtime version of the iterative Y-chart dataflow-based design methodology, where both energy and latency are optimized, SPiDER runtime resource manager has been selected. This tool, also developed at INSA, is the runtime counterpart of PREESM.

SPiDER was created with the idea of supporting reconfigurable PiSDF dataflow applications developed with PREESM design framework. This reconfiguration is represented by changes in the application parameters. For example, using the example shown in Figure 3-5, the number of slices can change so as to modify, at runtime, the parallelism of the application.

The internal structure of SPiDER is divided into different runtime managers: a GRT and several LRTs. The GRT is in charge of analyzing the PiSDF graph, performing the mapping and scheduling of the application over the different available $\mathrm{PE} s$ and distributing the work. It should be noted that those PEs can be either LRTs or the GRT itself. The LRTs, are deployed over the rest of PEs as lightweight processes with the only task of executing the actors they receive.

To graphically represent the behavior of this approach, Figure 3-10 gathers the execution scheme. First, the GRT, which is displayed as the Master process, analyzes the graph, maps and schedules the different actors composing the graph $\mathbf{0}$. Once the distribution is obtained, the GRT sends jobs to the different LRTs 2, which are represented as Slave processes. Each LRT, then, executes its corresponding actors 3 when the necessary data tokens are available in the pool of FIFOs 4 . After every actor execution, the LRT 3 send back the new values of the dynamic parameters $\mathbf{5}$ and trace information for monitoring and debugging purposes 6 .

Finally, one important aspect of SPiDER is that, currently, it only supports multicore architectures based on pthreads, but not the MPPA many-core platform. 


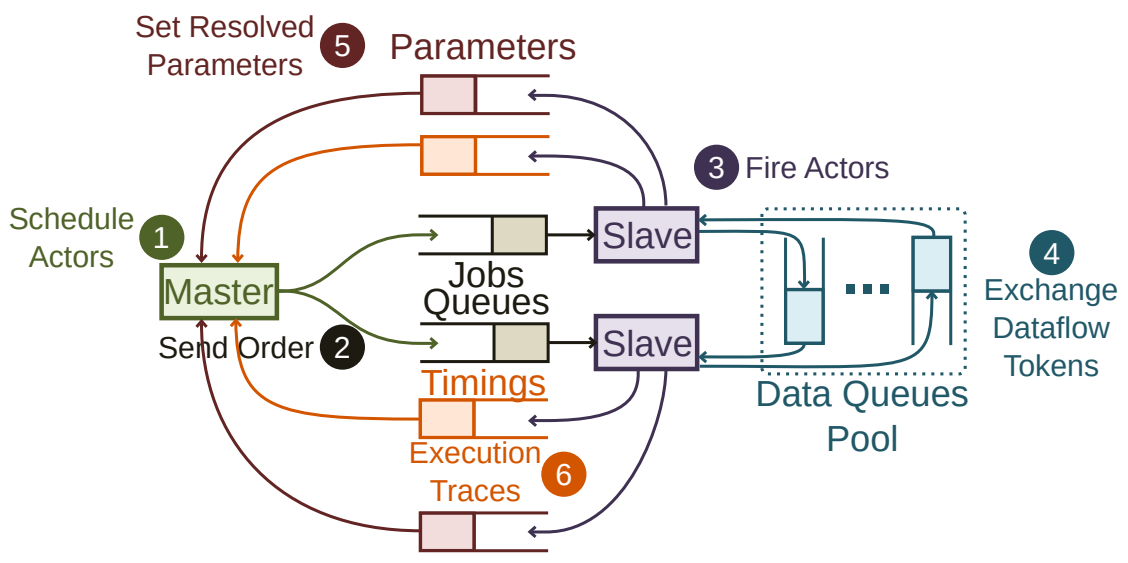

Figure 3-10: SPiDER runtime structure

Analyzing SPiDER from the point of view of extending its support to new architectures, its workflow can be separated in 3 blocks: the GRT, the LRTs and the communication among GRT and LRTs. The GRT is an architecture-independent manager, whilst the LRT 3 and the communication protocols are defined for each target architecture. Consequently, to include the MPPA among the supported architectures, both the LRT and the communication protocols should be implemented.

However, a new version of SPiDER-called SPiDER-2- has been envisioned during the development of this $\mathrm{PhD}$, and released during the writing of this document. This new version unifies both GRT and LRT to make both of them architectureindependent, hence minimizing the platform migration process to the definition of the communication protocols. This complete restructuring makes obsolete all the platform support done for the standard SPiDER.

In this context, two options were considered: to support the MPPA on the first version of SPiDER or to prioritize an architecture-independent approach during the integration of the runtime optimization loop within SPiDER. The former would imply the possibility of validating the optimization loop on the MPPA, but would make the work obsolete once SPiDER-2 is released. The latter would entail an extra effort but 1) porting the optimization loop onto SPiDER-2 would be straightforward; and 2) it would be applicable not only to pthreads platforms, but also to other architectures whose support is included within SPiDER-2 in the future, e.g., the MPPA itself.

As a result, to avoid already foreseen obsolescences, it has been decided to prioritize the architecture-independent approach. Since the MPPA platform support will not be addressed in this work, the validation of the energy-aware runtime optimization loop will be performed on a pthreads $\mathrm{x} 86$ architecture. Later, the loop will be simulated on the MPPA to evaluate the potential benefits that could be achieved. 


\subsection{Discussion}

This chapter has analyzed the different research opportunities related to the development of highly demanding applications onto multi-/many-core architectures when energy consumption is one of the requirements to be optimized. To tackle this problematic, an iterative Y-chart dataflow-based design methodology have been selected, which has been particularized as an energy-aware optimization loop. The definition of this loop, which embeds 3 blocks -monitoring, KPI estimation and multi-objective refinement- in the standard Y-chart design flow, is one of the original contributions of this $\mathrm{PhD}$.

Regarding the monitoring block, a new monitoring infrastructure called PAPIFY will be developed and explained in Chapter 4. PAPIFY will retrieve PAPI information following a Y-chart dataflow approach when profiling applications, i.e., the monitoring will be configured for each actor and each $\mathrm{PE}$. Consequently, versatile monitoring configurations on heterogeneous architectures will be supported.

In the case of the KPI estimation, since energy consumption has been selected as the $\mathrm{KPI}$ to be optimized in this $\mathrm{PhD}$, a new application-agnostic modeling methodology is introduced in Chapter 5 . This methodology has been proposed under the hypothesis of dividing the energy consumption in 3 contributions: resourceactive, communication and computation. Additionally, the model will be based on information that can be retrieved at runtime; hence, it will be possible to estimate the energy consumption at both design time and runtime.

To define the multi-objective refinement block, an energy-aware tuning algorithm explained in Section 6.2- is proposed when considering energy consumption as one of the requirements to be minimized. This algorithm is a searching mechanism that looks for the system configuration that minimizes the energy consumption while fulfilling a performance objective. To increase the portability of the tuning algorithm, it will be built surrounding the standard latency-based mapping/scheduling algorithms that already exist in most dataflow design frameworks.

Finally, to validate the methodology, two frameworks have been selected to implement the energy-aware optimization loop: PREESM and SPiDER. The former will prove the methodology at design time (Section 6.3), focusing on enhancing DSE capabilities with energy-awareness. The latter will optimize the system energy consumption at runtime Section 6.4). The results associated to this analysis are gathered in Chapter 7 . 


\section{MONITORING - PAPIFY}

This chapter presents the work associated to the monitoring block included in the energy-aware optimization loop (Figure 4-1). After analyzing the state of the art related to application monitoring tools -Section 2.4-, it has been decided to develop a new monitoring infrastructure called PAPIFY ${ }^{1}$. With this approach, dataflow-oriented applications can be instrumented when running on heterogeneous platforms, since with this infrastructure the monitoring is transparently managed at runtime.

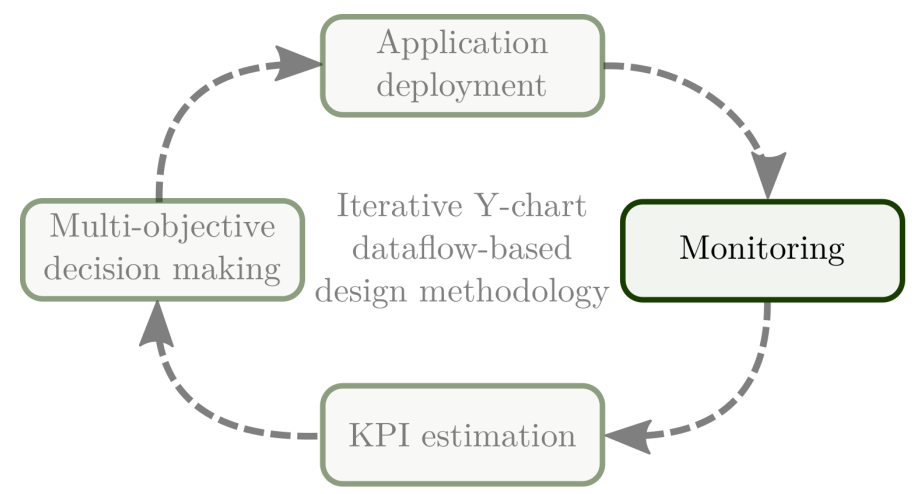

Figure 4-1: Iterative Y-chart dataflow-based design methodology - Monitoring

Along this chapter, first, a brief introduction of the tool and an example that is used to guide the rest of the explanation are provided in Section 4.1. Later, the monitoring configuration, which is associated to the design time, is detailed in Section 4.2, After this, Section 4.3 presents the runtime management of the monitoring configurations. Then, in Section 4.4, a complementary tool called PAPIFY-VIEWER, which aims at graphically representing the monitoring data, is explained. Finally, a brief summary of the main ideas is provided in Section 4.5 .

\footnotetext{
${ }^{1}$ Code available at https://gitlab.citsem.upm.es/papify/papify
} 


\subsection{PAPIFY introduction}

As presented in Section 2.4. current monitoring methodologies focus either on retrieving PMC-based hardware information or on profiling applications from a system-level point of view. Consequently, developers have to select between having a deep understanding of the system behavior or obtaining a system-level overview of their system. Likewise, even if developers decide to follow the former approach, in most of the cases, the system has to be profiled in a homogeneous way.

In this PhD, PAPIFY [Madronal'19a] is proposed as a monitoring solution merging the benefits of a dataflow Y-chart approach and PAPI library, which retrieves PMC information and links it to the application tasks, even in a heterogeneous scenario.

Specifically, PAPIFY was born to deal with different monitoring configurations for each application actor and to support different profiling configurations depending on the resource executing the actor, specially when a heterogeneous architecture is considered. In this situation, the actor monitoring will be valid even if the dataflow application is not statically deployed and, for instance, a runtime resource manager decides to dynamically remap one actor on a $\mathrm{PE}$ of a different nature.

To represent the idea PAPIFY wants to address, an example of a system with the following characteristics and behavior is considered. In this example, from the perspective of the monitoring infrastructure, only PAPIFY would be able to successfully retrieve the desired profiling data in a single execution.

\section{- System characteristics}

- Application: 4 actors named Read_YUV, Sobel1, Sobel2 and display. Both $\overline{\text { Sobel actors }}$ can be executed in parallel.

- Architecture: a PE of type x86 and another PE of type FPGA.

\section{- Monitoring configuration ${ }^{2}$}

- Read_YUV: actor timing and PAPI_TOT_INS when executed on x86 and nothing when executed on the FPGA

- Sobel: actor timing and PAPI_L1_DCM when executed on x86 and FPGA_TOTAL_CYCLES when executed on the FPGA.

- display: no monitoring.

\section{- Application behavior}

- First iteration: all the actors are mapped on the x86 PE.

- Second iteration: Sobel2 is remapped on the FPGA|PE.

\footnotetext{
${ }^{2}$ Refer to Subsection 2.4.1 for more details about PAPI events
} 
To graphically analyze the example, it has been represented in Figure 4-2, Actors have been represented as rectangles with their names inside and $\overline{\mathrm{PE}} \beta$ are plotted in horizontal lines, where each iteration is separated with a vertical dashed line. Additionally, the starting point of each actor has been marked with a letter, going from $A$ to $H$. Finally, the different monitoring configurations have been represented using numbers: in $\mathbf{0}$, time and PAPI_TOT_INS are monitored; in 2 , time and PAPI_L1_DCM are profiled and, in 3, only FPGA_TOTAL_CYCLES event is retrieved. This example will be continuously referenced along the chapter to explain the tool.

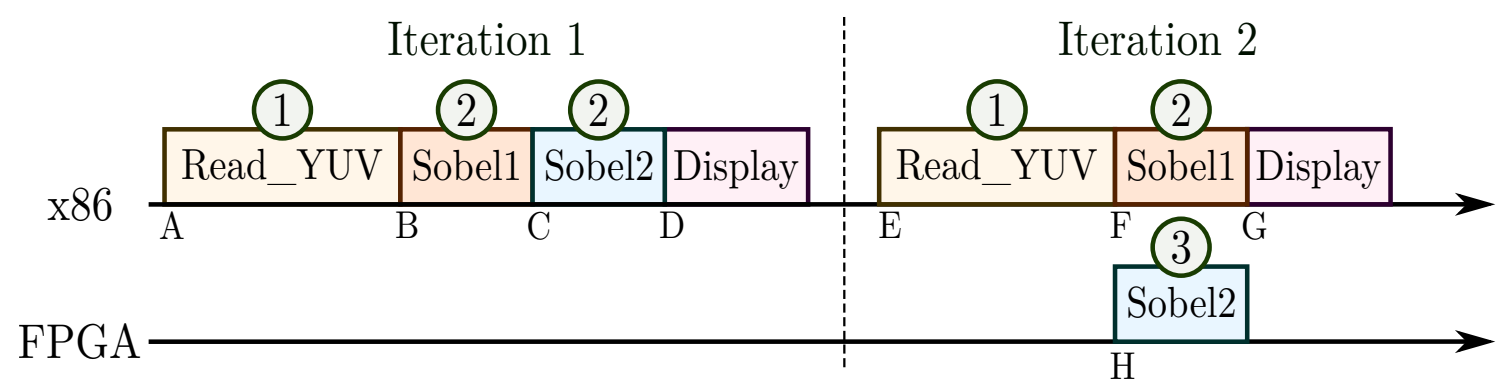

Figure 4-2: PAPIFY use-case example. Versatile monitoring on a heterogeneous architecture

As already mentioned before, PAPIFY toolbox has been built on top of PAPI -see Figure 4-3-. As a reminder, PAPI uses two levels of abstraction to retrieve hardware PMC information: a low-level composed of architecture-dependent components; and a high-level API to unify its usage. In this line, PAPIFY extends this abstraction from the point of view of the details of both the dataflow application and the architecture where the application is executed. To do so, a dedicated library called eventLib has been developed as an instrumental contribution of this $\mathrm{PhD}$ to manage, at runtime, the different monitoring configurations that users can configure during the application design. Hence, eventLib simplifies the usage of PAPI in the dataflow context.

During the following sections, the different parts in which PAPIFY is divided will be explained together with its integration with both PREESM and SPiDER tools. Although PAPIFY can be used independently, the explanation is carried out this way to show how it can help in real Y-chart dataflow design frameworks.

- Papify-DT: to configure the monitoring and instrument the application code.

- Papify-RT: to manage the different configurations and apply the one corresponding to each actor-PEpair.

- PAPIfy-Viewer: to display and analyze the PMChardware information. 


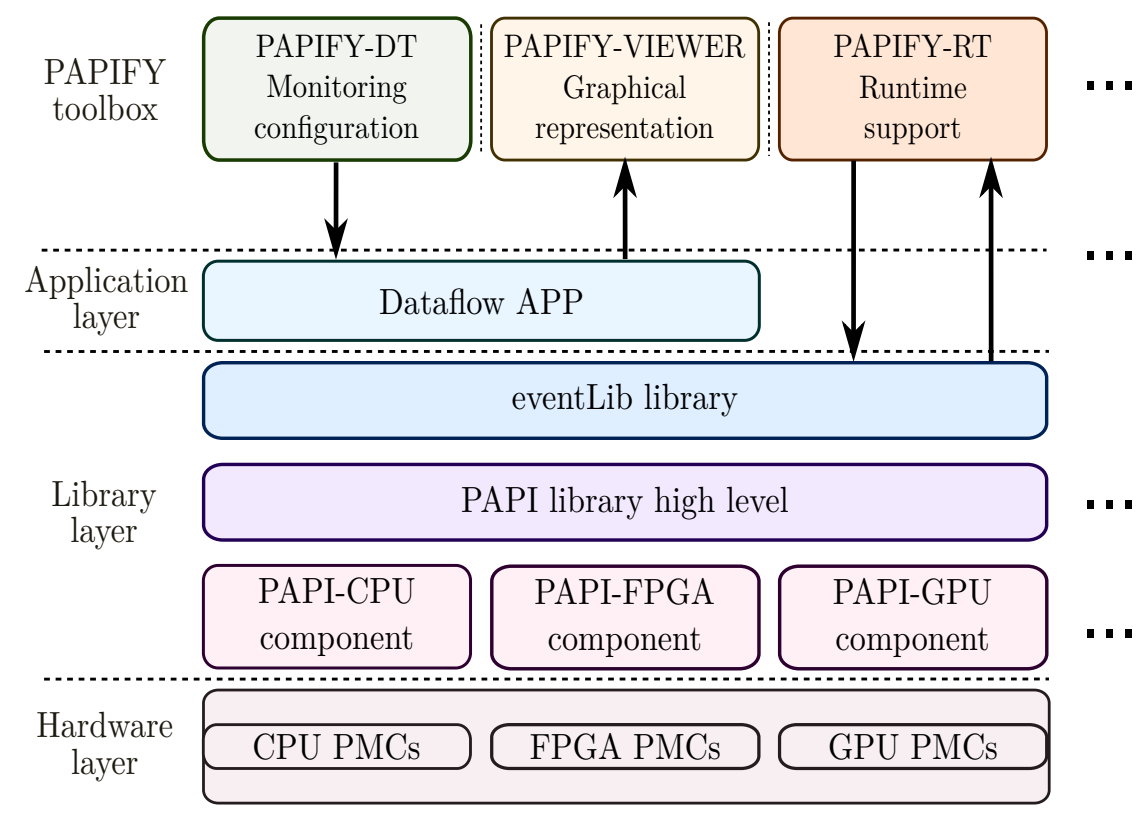

Figure 4-3: PAPIFY diagram

\subsection{PAPIFY configuration - PaPIFY-DT}

From the point of view of the design time, PAPIFY-DT role is to ease the PMC-based monitoring configuration of dataflow applications. In this regard, the tasks to be completed at design time cover not only the definition of the events to be monitored for each actor when being executed on each $\mathrm{PE}$, but also the code instrumentation.

In order to deal with these aspects, the functionalities within eventLib library have been organized in 4 different groups: library initialization, monitoring configuration, monitoring body and library termination.

Concerning the library initialization, eventLib contains event_init_multiplex() and event_init (). These two functions will initialize both PAPI and eventLib libraries considering whether the multiplexing ${ }^{3}$ capability provided by PAPI is enabled or not, respectively. These functions have no parameters and they are completely independent from the application and the architecture being used.

From the configuration point of view, the library is also composed of only two functions: configure_papify_actor() and configure_papify_PE(). These two functions are in charge of isolating actor and $\mathrm{PE}$ configuration, following the Y-chart dataflow design flow proposed as one of the PAPIFY bases.

- configure_papify_actor(): the inputs of this function are 1) the actor name -actorName-, 2) the events that developers want to monitor for this actor -

\footnotetext{
${ }^{3}$ Refer to Lim'14 Dongarra'01 for more information about event multiplexing within PAPI
} 
eventNames-, 3) the number of total events being monitored -numEvents, 4) the names of the PAPI components where the events will be found ActorPAPIComponentNames-, 5) the total number of different PAPI components with a valid monitoring -numConfigs- and 6) unique identifiers associated with each different monitoring configuration -eventSetIds-. Based on this information, the function sets up a PapifyInfo variable including the monitoring configurations associated to the actor and their unique ids.

- configure_papify_PE(): using as input parameters 1) the name of the PE -PEName-, 2) the PAPI component developers want to associate to the PE PEPAPIComponentName - and 3) a unique identifier -PEId-, this function links the PE with the PAPI component and the unique $i d$.

Regarding the body of the application monitoring, PAPIFY has been built considering the possibility of monitoring just the execution time of the application to retrieve only hardware PMC information or to profile the system based on both data sources. Additionally, a function to store all the monitoring information is also provided so as to analyze it with the PAPIFY-VIEWER tool -which will be explained in Section 4.4-. In all the cases, the input parameters are the PapifyInfo variable associated to the actor and the unique $i d$ of the PE executing it.

- event_start_papify_timing(): stores the starting time of the actor.

- event_stop_papify_timing(): stores the finishing time of the actor.

- event_start(): starts counting the events associated to the actor that are available in the specific $\mathrm{PE}$ executing it. The internal behavior of this function will be explained in Section 4.3 .

- event_stop(): finishes the count of events.

- event_write_file(): dumps the monitoring information on a CSV file.

Finally, in order to avoid memory leakages in both PAPI and eventLib libraries, a function called event_destroy() is provided. This function will deal with tasks related to stopping all the counting (if any), freeing the memory that has been reserved and shutting down both libraries.

Code template 4.1 exemplifies the instrumentation of Read_YUV actor on an x86 PE-extracted from Figure 4-2- using eventLib. It should be considered that, in this example, the standard $\mathrm{PAPI}$ component for CPU, which is called perf_event, has been used. This example shows that, as mentioned before, the instrumentation of the code is based on 9 function calls: one to initialize PAPI and eventLib libraries (line 2), two to configure the system monitoring (lines 13-14), 4 to control the actual monitoring (lines 16-17 and lines 21-22), one to save the PMC data (line 24) and one to shut down both libraries (line 26). 
Code template 4.1: Instrumented code using eventLib

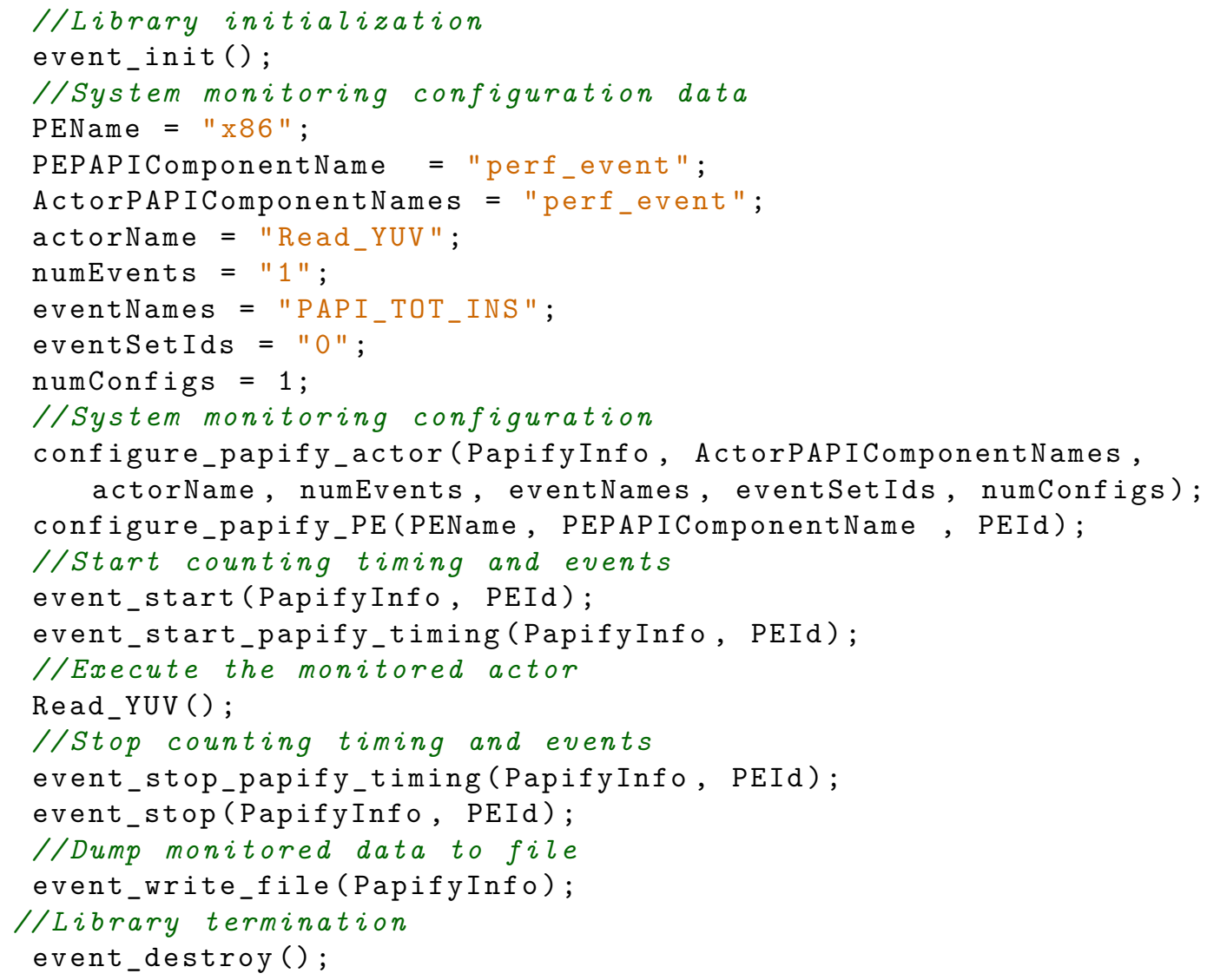

Once eventLib structure has been explained, the integration of PAPIFY with PREESM interface is going to be explained, as it is mainly devoted to ease both monitoring configuration and code instrumentation ${ }^{4}$. This integration has been carried out to provide the developer with 1) a graphical interface to configure application monitoring and 2) an automatic generation of instrumented code.

Figure 4-4 shows the configuration required to perform the monitoring exemplified in Figure 4-2, As can be seen, the monitoring of dataflow applications using PAPIFY through PREESM is divided into 3 sections.

- First, the PAPI monitoring information of the target architecture has to be loaded using the XML file generated by executing papi_xml_event_info in the PAPIFY file path section. As this information is generated on the target architecture, the development of the application can be easily done in a workstation.

- Secondly, the linkage between PAPI component and PE type is done in the PAPIFY PE configuration section. This part has been developed considering that the target platform might be heterogeneous, as shows the work presented

\footnotetext{
${ }^{4}$ Tutorial on how to use PAPIFY from PREESM available at https://preesm.github.io/tutos/papify/
} 
in [Fanni'19]. The one-to-one association is done through a table where the available PAPI components are shown in the first column and the different types of $\mathrm{PE}$ present in the target architecture are shown in the first row.

- Finally, in the PAPIFY actor configuration section, the user selects the events that need to be monitored for each actor. In this case, actors correspond to rows, and the available events, gathered from the available PAPI components in the target platform, are linked to columns. To sum up, developers select the events they want to monitor independently of the PAPI component they belong to.

\section{PAPIFY}

- PAPIFY file path

Enter an xml file path that contains the output of the papi_xml_event_info command executed within the target platform. PAPI components and their associated events will be automatically added to the selection options.

Edit file /sobel-morpho/Code/PAPI_info_Heterogeneous.xml

Browse

PAPIFY PE configuration

Each SLAM processing element instance needs to be associated with its corresponding PAPI component

\begin{tabular}{l|l|l} 
Component type $\backslash$ PE type & X86 & FPGA
\end{tabular}

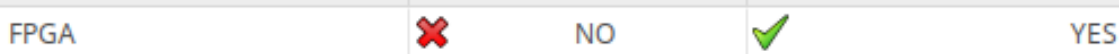

perf_event $\forall$ YES NO

- PAPIFY actor configuration

Each actor needs to be associated with its corresponding event(s)

Actor name I Event name

Timing

FPGA_TOTAL_CYCLES

PAPI_L1_DCM

PAPI_TOT_INS

- $\square$ sobel_morpho

Read_YUV

display

Sobel

\begin{tabular}{|c|c|}
\hline $\mathfrak{z}$ & NO \\
\hline$\nabla$ & YES \\
\hline$\tilde{3}$ & NO \\
\hline$\nabla$ & YES \\
\hline
\end{tabular}

\begin{tabular}{|c|c|c|c|}
\hline జ & NO & 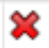 & NO \\
\hline జ & No & $\approx$ & NO \\
\hline 2 & NO & $\approx$ & NO \\
\hline$\checkmark$ & YES & $\nabla$ & YES \\
\hline
\end{tabular}

$\begin{array}{ll}\approx & \text { NO } \\ \mathbb{4} & \text { YES } \\ \mathbb{4} & \text { NO }\end{array}$

Figure 4-4: PAPIFY PREESM user interface

After the developer has configured the monitoring of the application, during the code generation task of PREESM, this information is analyzed so as to provide an instrumented code already embedding all the required function calls to eventLib. Consequently, thanks to this work, the developer is provided with a methodology to easily configure the monitoring of heterogeneous (or not) dataflow applications and automatically generate instrumented code. 


\subsection{PAPIFY runtime support - PAPIFY-RT}

The objective of PAPIFY-RT is to transparently apply and manage monitoring configurations at runtime. In this sense, the main task consists in selecting the actor configuration that should be used on the PE executing it.

To carry out this task, it should be considered that, as mentioned before, the PE and actor monitoring configurations are isolated from each other. Specifically, while the PE is linked with a specific PAPI component, the actor monitoring is associated only to PAPI events, independently from the PE executing the actor and the PAPI component the events belong to. The management of this variability is embedded within PAPIFY architecture and controlled in the event_start () function call.

Specifically, this function follows Algorithm 4-1. As can be seen, 3 different states are defined to efficiently manage the monitoring information at runtime:

- FirstConfig: if the actor has never been monitored in that $\mathrm{PE}$, its monitoring configuration -the so-called eventSet-is created, stored, launched and the event values are read. This happens at points $A, B$ and $H$ in Figure 4-2.

- KeepCounting: if the actor eventSet is the one being currently monitored in that $\mathrm{PE}$, the event values are read. This is the case of point $C$ in Figure 4-2.

- FastSwitching if the actor eventSet is not the one being currently monitored but it has been previously configured in that $\mathrm{PE}$, the current eventSet is stopped, the new one is launched and the event values are read. This situation is the one linked to points $E$ and $F$ in Figure 4-2.

It should be highlighted that, during the event_stop () function, the eventSet is not stopped, but the event values are read and the total amount of event occurrences is computed by differences with the ones read during event_start () function.

These strategies have been considered so as to reduce as much as possible the overhead of the monitoring tool. As can be noticed when comparing [Madroñal'18] and [Madronal'19a], using this approach, the performance overhead, in the worst case, is reduced from approximately a $67 \%$ to a $10 \%$.

After analyzing the internal behavior of PAPIFY-RT, it can be deduced that, currently, PAPIFY can monitor both static and dynamic dataflow applications. In the static case, every configuration will be carried out during the first iteration and, after this, no FirstConfig will be needed anymore. On the contrary, in a dynamic dataflow context, a resource manager like, for example, SPiDER, can remap and reschedule the application on-the-fly. In this situation, a FirstConfig can happen at any time, as the resource manager can decide in any iteration to map an actor on a $\mathrm{PE}$ that never executed it before, as represented in the second iteration of Figure 4-2 with Sobel2. 


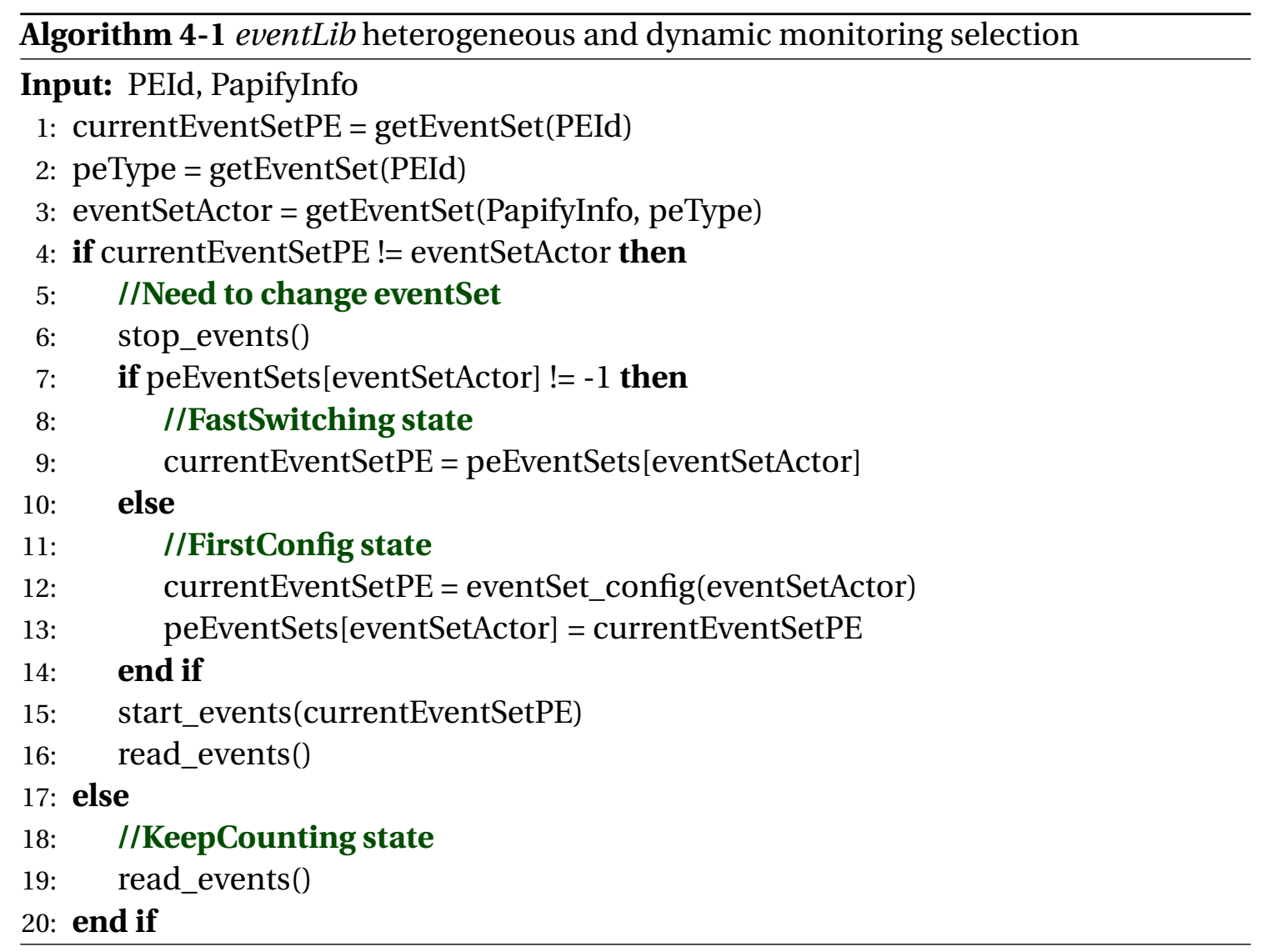

In this line, including PAPIFY in the SPiDER work-flow provides this tool with PMC-based monitoring, which, in turn, provides self-awareness of the current system status. Specifically, as shown in Figure 4-5. PAPIFY has been embedded within the slave processes and all the PMC information is retrieved by the master process. This procedure, together with the use of this performance information to refine on-the-fly mapping and scheduling decisions, will be detailed in Chapter 6 .

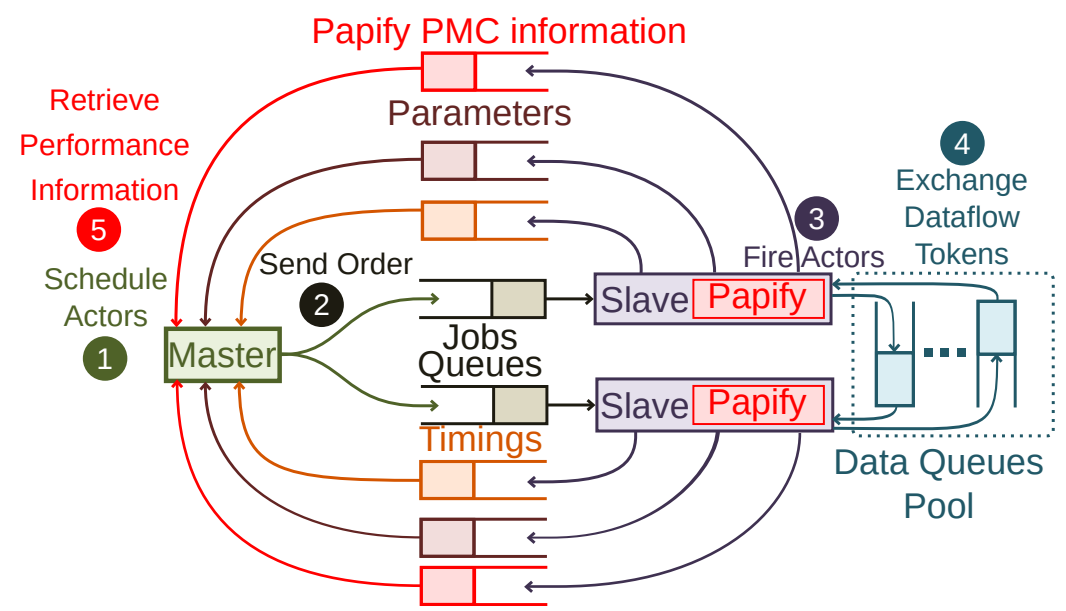

Figure 4-5: PAPIFY-SPIDER runtime structure 


\subsection{Papify-Viewer}

PAPIFY-VIEWER aims at easing the analysis of the monitoring data by means of a visualization tool coded in Python. Moreover, this tool can be either used during the execution of the application or in post-execution time. However, its use at runtime is not recommended in a final application, as its overhead is not negligible |Madronal'19al. PAPIFY-VIEwER can represent the information in two different ways: actor PE workload distribution and event-actor analysis.

Figure 4-6 shows an example of the actor-PE workload distribution view, where each $\mathrm{PE}$ is represented in the vertical axis, while the horizontal axis is a time-line. Likewise, colored rectangles represent the time slot taken by actors when being executed on each $\mathrm{PE}$, and the actor to color legend is included on the image right side.

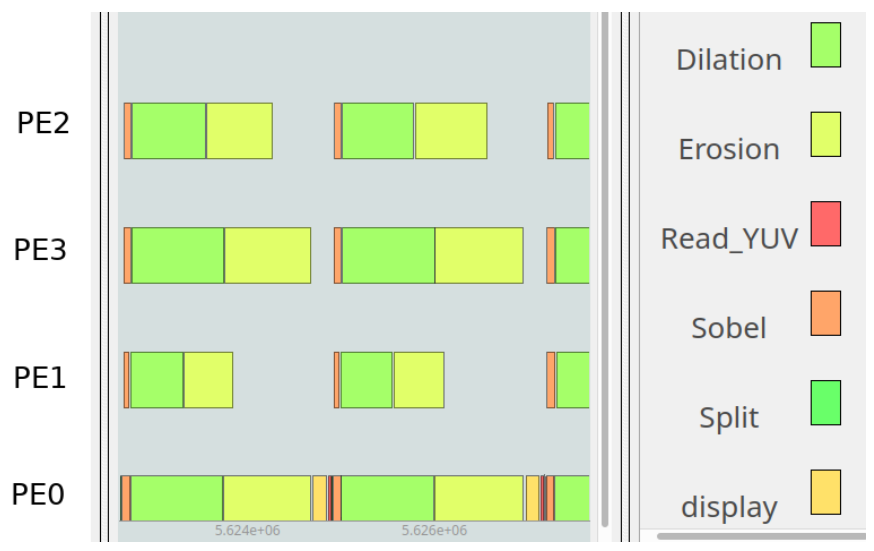

Figure 4-6: PAPIFY-VIEWER workload distribution view

Figure 4-7 displays event-actor analysis view. In this case, each actor is represented in the horizontal axis. The vertical one presents an average of the events, computed with all the times each actor is fired. Furthermore, in order to change the event being displayed, a list with the monitored events is shown on the right side of the view.

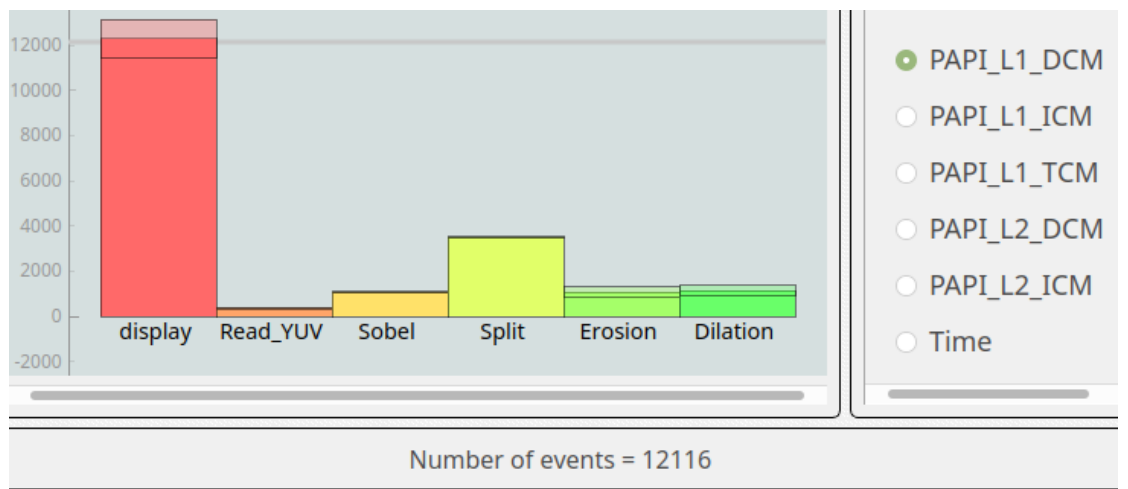

Figure 4-7: PAPIFY-VIEWER hardware utilization for PAPI_L1_DCM event view 


\subsection{Discussion}

Along this chapter, PAPIFY monitoring infrastructure has been detailed focusing on each of the pieces composing it: design time, runtime and graphical representation. This open-source tool is one of the instrumental contributions of this $\mathrm{PhD}$ work, since it has been used to characterize applications in a dataflow-oriented approach, hence providing individualized timing and PMC information for each actor composing the application when running on each architecture $\mathrm{PE}$.

This tool is extensively used during Chapter 7 to characterize the dataflow version of the HSI cancer detection application using both PREESM and SPiDER. In this regard, the benefits obtained when using PAPIFY are demonstrated in two sets of experiments. The former focuses on profiling the application in terms of execution time so as to enhance the latency-based mapping/scheduling carried out by PREESM and SPiDER, detailed in Subsection 7.3.3 and Subsection 7.4.2, respectively. The latter employs PMC information to infer energy consumption based on the modeling approach explained in Chapter 5. The results associated to the second analysis are exposed in Subsection 7.3.4 for design time and in Subsection 7.4.3 for runtime monitoring. 



\section{ENERGY ESTIMATION}

In this chapter, the work associated to the KPI estimation block of the iterative design methodology is detailed (Figure 5-1). Specifically, the KPI that will be modeled is the platform energy consumption, since this PhD's objective is to optimize this figure.

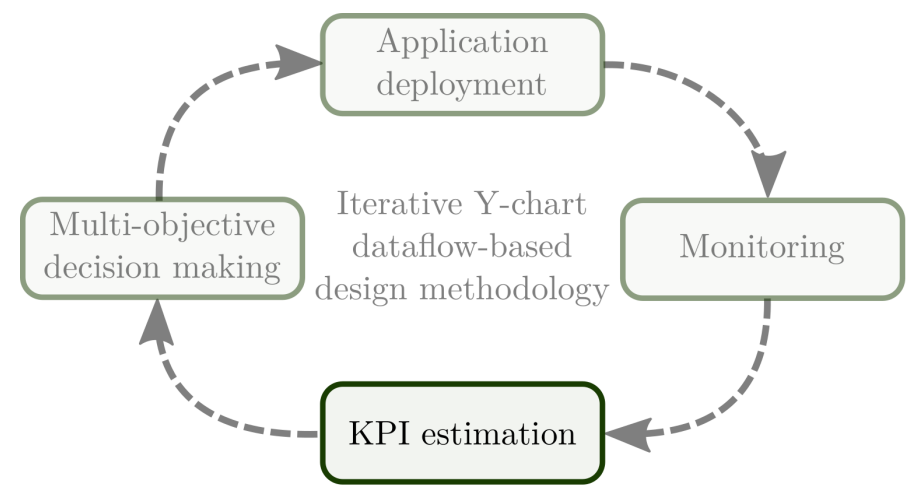

Figure 5-1: Iterative Y-chart dataflow-based design methodology - KPI estimation

In this regard, the proposed modeling methodology -introduced in Subsection 3.2.3 - is built considering a high-level approach that aims at being applicable on different architectures. Precisely, in this PhD, a model of the MPPA-256-N is built, since it is the target architecture of the selected use-case. As explained in the overview provided in Section 5.1, this methodology divides the energy consumption of the platform into 3 contributions, each of them with a dedicated section in this chapter: resource-active (Section 5.4), communication (Section 5.5) and computation (Section 5.6). Subsequently, the modeling of each contribution is divided into 3 parts: model definition, $\mu$ benchmarks creation ${ }^{1}$ and model extraction.

After this, the platform model is summarized in Section 5.7. Additionally, this section presents a validation of the energy consumption estimations using a General Matrix Multiplication (GeMM) application.

It should be noted that, before analyzing the energy consumption itself, some preliminary steps must be fulfilled. First, an analysis of a set of different power measurement procedures that can be used on the MPPA is discussed in Section 5.2 and the most suitable one is selected. Then, Section 5.3 presents the work related to the PAPI library support on the MPPA as accessing PMC information is required to build the computation contribution model.

Finally, Section 5.8 summarizes the main ideas presented in this chapter.

\footnotetext{
${ }^{1}$ The benchmarks can be found in https://gitlab.citsem.upm.es/dmadronal/kernelsCode
} 


\subsection{Modeling methodology overview}

In this section, a methodology to build energy consumption models is proposed. These models are constrained in 2 senses: they have to be 1) simple and 2) generic. A simple model can be used to estimate energy consumption either at runtime or in an iterative application design methodology. In turn, a generic one can represent the energy consumed by the utilization of the different platform resources, which leads to the development of an application-agnostic energy consumption model.

In this line, in order to develop a model that can be applied to any application, it has been decided to divide the energy that is consumed by the architecture into 3 contributions, where each of them models the energy consumption due to a different activity/resource utilization. As a result, the energy consumed by the platform (Energ $y_{\text {platform }}$ ) will follow Equation 5-1, where the total contribution has been divided in 3 isolated parts, as graphically represented in Figure 5-2.

Energ $y_{\text {platform }}[\mathrm{J}]=$ Energ $y_{\text {resource-active }}+$ Energ $y_{\text {comms }}+$ Energ $y_{\text {comp }}$

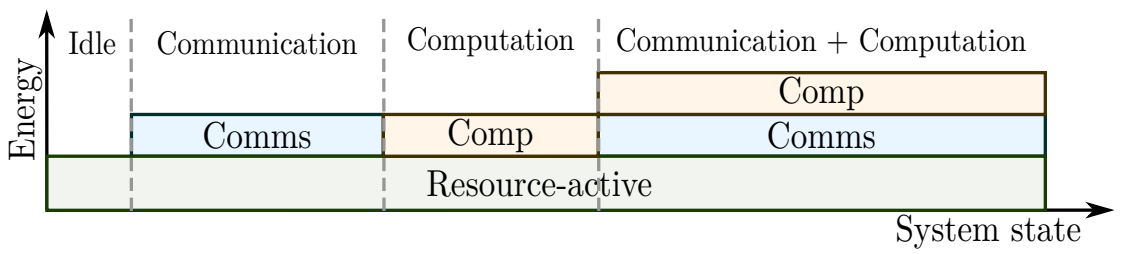

Figure 5-2: Representation of the proposed energy consumption model

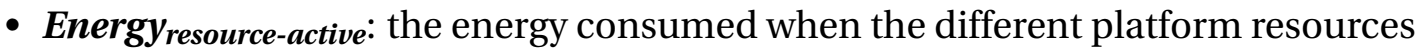
are switched on, being considered the baseline of the power consumed by the platform. This part of the model is detailed in Section 5.4.

- Energy $y_{\text {comms: }}$ : the energy consumed when transmitting data among resources. This energy consumption contribution is explained in Section 5.5.

- Energy $y_{\text {comp: }}$ the energy spent when the platform resources are processing data. This energy source is deeply analyzed in Section 5.6.

As explained in Subsection 2.5.2, specific $\mu$ benchmarks will be developed in each case, stressing the architecture in such a way that each contribution can be isolated from each other. During the execution of these benchmarks, in order to build a model that supports energy estimations of real executions, the information selected to be monitored needs to be 1) accessible at runtime and 2) representative enough to obtain 
accurate estimations. Consequently, for example, in the case of the computation, it has been decided to use PMC hardware information retrieved through PAPI.

In this context, the MPPA-256-N platform will be used during the chapter to exemplify the methodology, since it is the platform used in the PhD use-case. This platform, as mentioned in Subsection 2.2.2, presents different levels of hierarchy in terms of computational resources and memory. To guide the explanation of the modeling methodology, Table 5-1 gathers information and simplified block diagrams representing the parts of the MPPA that will be used during each part of the model.

Table 5-1: MPPA block diagrams used to develop the energy consumption model

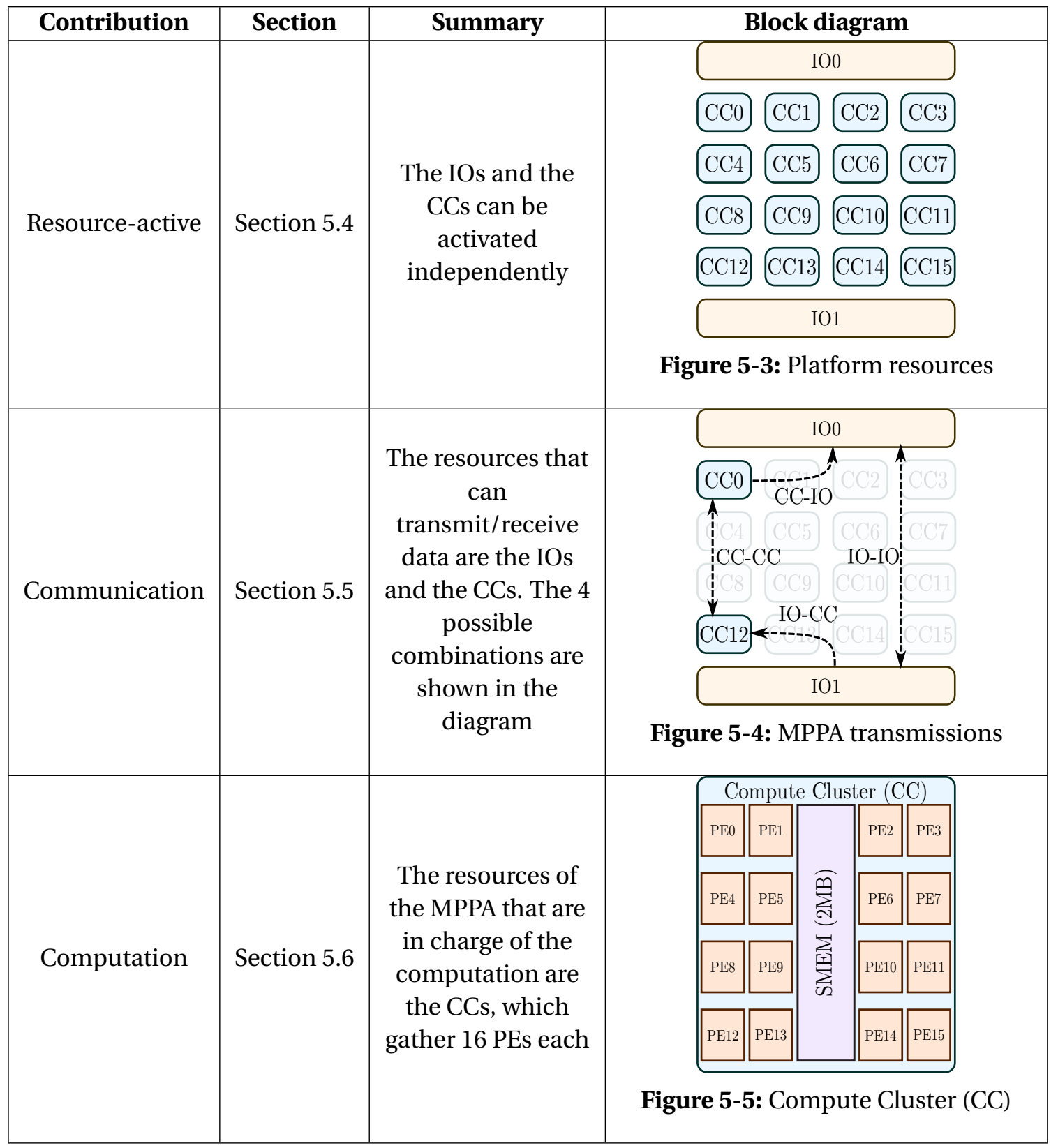




\subsection{Power measurement procedure}

The first step consists in analyzing 3 different tools to measure the real power consumption of the platform. This stage is crucial, as the model will consider these measurements the ground truth. As highlighted in [Roux'17], the changes in the MPPA power consumption are really small, so this study will look for the procedure providing the highest resolution. The surveyed tools are:

- k1-power: the standard tool provided by Kalray to measure power consumption. It gives access to a set of sensors available on the board measuring voltage, current, power consumption and temperature.

- Oscilloscope: a WaveRunner 640Zi from Teledyne LeCroy plugged on a shunt resistor of $0.5 \mathrm{~m} \Omega$, which already exists on the board.

- Multimeter: a 34450A multimeter from Keysight (Agilent) plugged on the aforementioned shunt resistor.

\section{k1-power}

This tool provides the user with access to sensors measuring the voltage, the current and the power consumption of the different resources existing in the board. Specifically, it can read simultaneously the power consumption of the DDR (memory used by the $\mathrm{IO}$ ) and the computational core (hereafter, Core) of the board, which includes the power consumed by the $\mathrm{CC}$, the computational part of the $\mathrm{IO}$ and the NoC. However, there is no sensor to measure the power consumption of the IO1 DDR. To be precise, $k 1$-power provides up to 50 samples per second, and a resolution of $100 \mathrm{~mW}$ and $1 \mathrm{~W}$ for the DDR power and the Core, respectively.

An example of the kl-power power consumption measurements is shown in Figure 5-6, where Core is represented in blue andDDR is depicted in red. Specifically, a matrix multiplication test where the active PEs increase in steps of 1 has been used. For each number of $\mathrm{PE} s$-starting with 1 and ending with 256-, a time slot of 60 seconds is shown in the figure. Additionally, a detailed version of this test is shown in Figure 5-7. where 64 different steps should be distinguished. For both figures, the ticks in the horizontal axis correspond to the starting point of the 60 seconds time slot, while the number represents the active $\mathrm{PE}$. As can be seen, small changes in the Core cannot be detected with $k 1$-power. That is, the measurement is not stable and it resembles noise, since it varies between the specific value and $\pm 1 \mathrm{~W}$. 


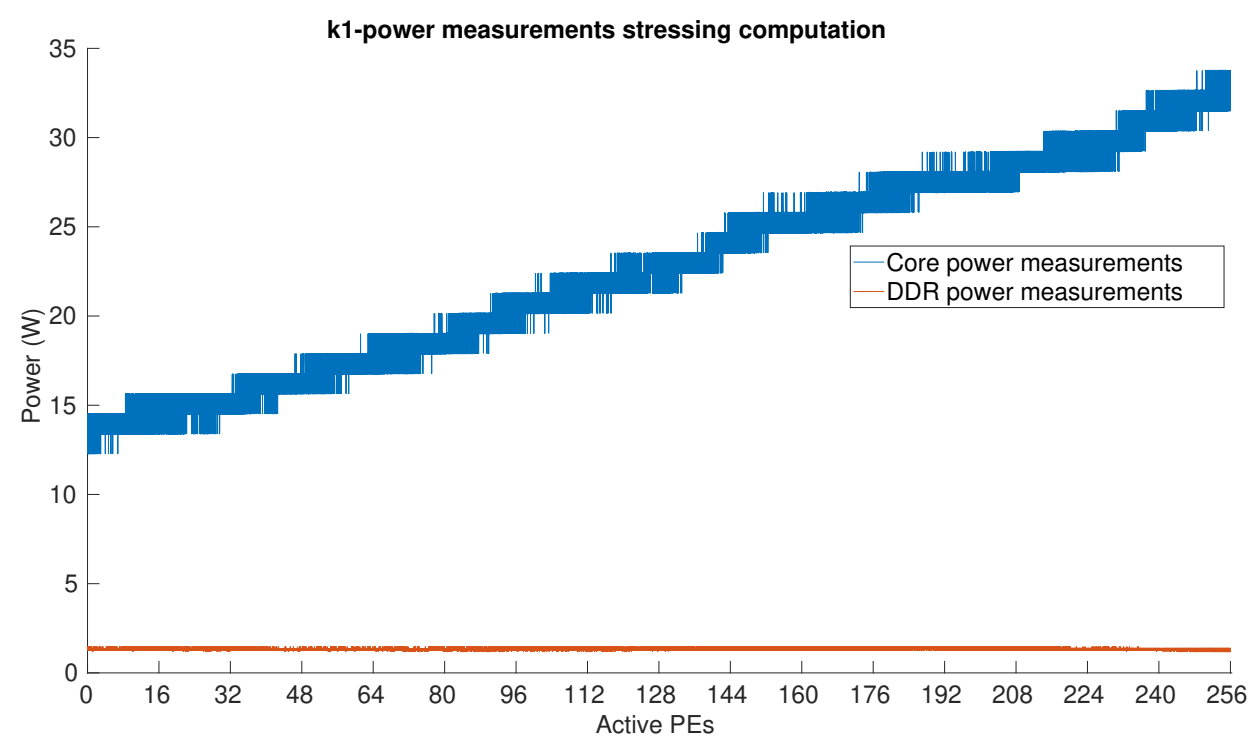

Figure 5-6: $k 1$-power measurements for matrix multiplication

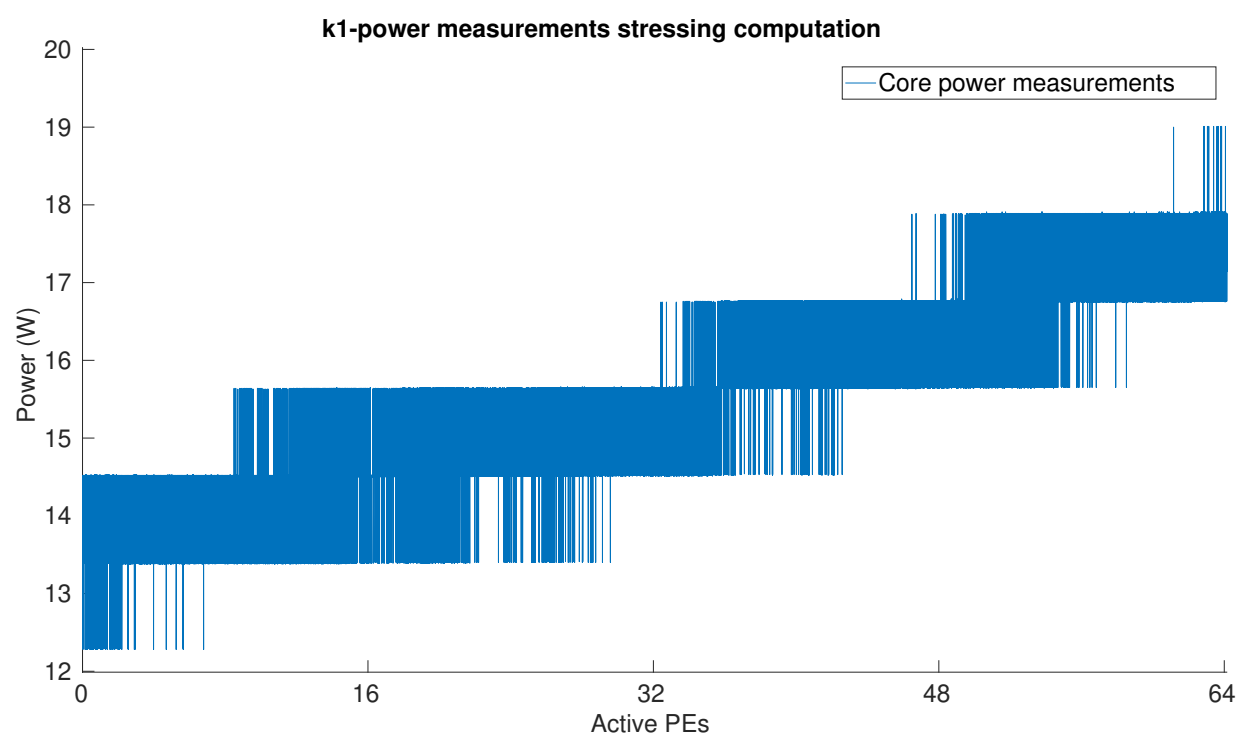

Figure 5-7: Detail of core kl-power measurements for matrix multiplication

\section{Oscilloscope}

Aiming at increasing the precision of the measurement, the use of an oscilloscope to measure the Core power has been considered. The oscilloscope has a maximum sampling rate of $40 \mathrm{GS} / \mathrm{s}$ and a resolution of $1 \mathrm{mV} / \mathrm{div}^{2}$. Since the oscilloscope is directly plugged onto a shunt resistor of $0.5 \mathrm{~m} \Omega$, which exists on the board to measure the Core

\footnotetext{
${ }^{2}$ WaveRunner 640Zi data-sheet: https://cdn.teledynelecroy.com/files/pdf/waverunner-6zidatasheet.pdf
} 
supply, only Core power consumption information can be retrieved. As a result, since the Core of the board has a power supply of $0.9 \mathrm{~V}$, in terms of power, the resolution corresponds to Equation 5-2, being even lower than the one obtained using k1-power.

$$
\text { Power }=(1 \mathrm{mV} / 0.5 \mathrm{~m} \Omega) * 0.9 \mathrm{~V}=1.8 \mathrm{~W}
$$

\section{Multimeter}

Finally, the use of the multimeter $34450 \mathrm{~A}^{3}$ from Keysight (Agilent) is evaluated. This multimeter is providing one sample per second and, in terms of resolution, it provides an accuracy of $1 \mu \mathrm{V}$. As the objective is to measure the voltage on the previously mentioned shunt resistor, the power resolution, in this case, would be the one represented in Equation 5-3.

$$
\text { Power }=(1 \mu \mathrm{V} / 0.5 \mathrm{~m} \Omega) * 0.9 \mathrm{~V}=18 \mathrm{~mW}
$$

In this case, to exemplify the use of this instrument to take the measurements, Figure 5-8 gathers the power information when launching the same test employed in Figure 5-6. As can be seen, the measurements are cleaner and, in the detailed version shown in Figure 5-9, the 64 steps can be distinguished, which means that, using this approach, the power consumption associated to the activation of one PE to compute the matrix multiplication can be discerned.

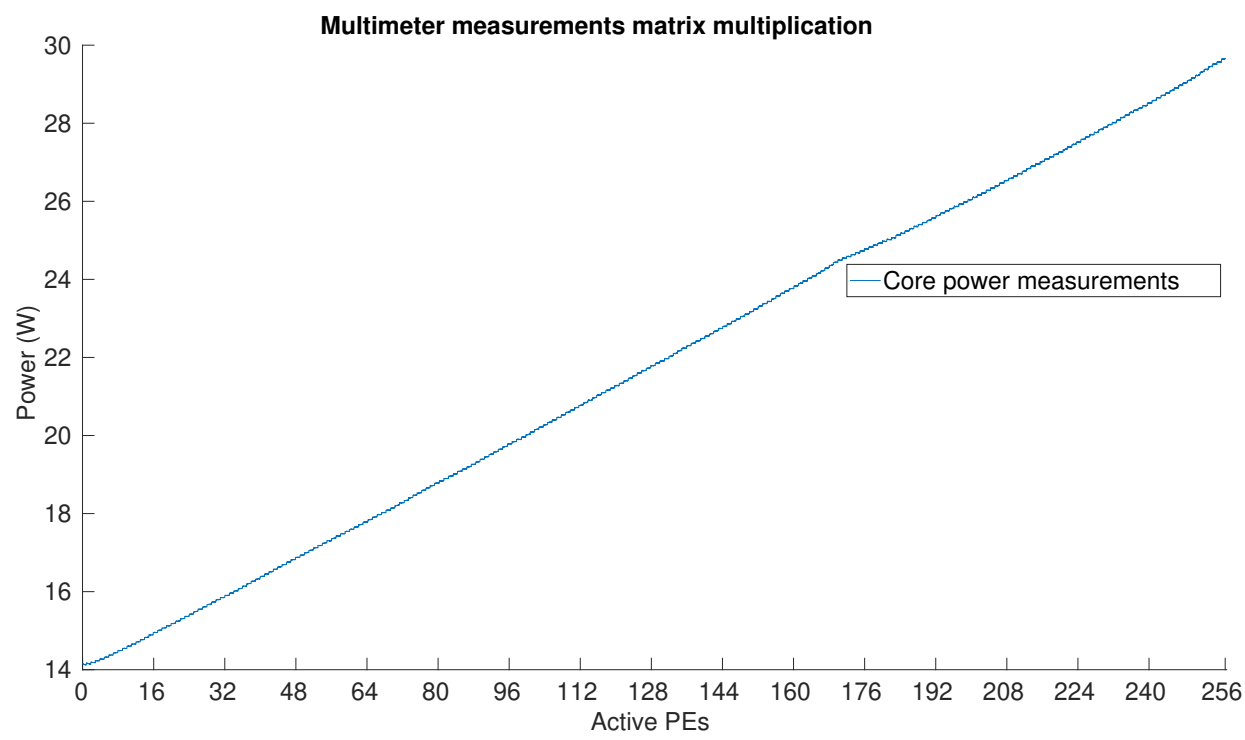

Figure 5-8: Multimeter measurements for matrix multiplication

\footnotetext{
${ }^{3}$ 34450A data-sheet: https://literature.cdn.keysight.com/litweb/pdf/5991-1133EN.pdf
} 


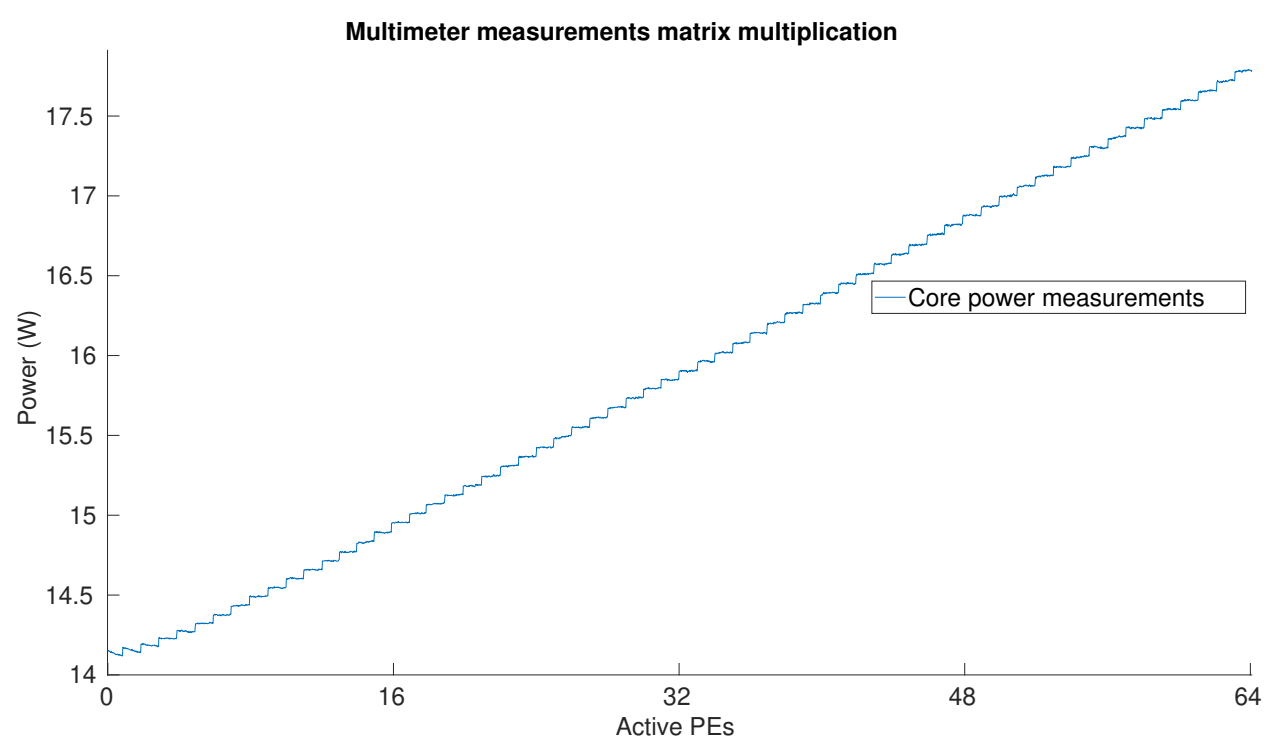

Figure 5-9: Detail of multimeter measurements for matrix multiplication

\section{Decision}

To decide which measurement procedure is the most suitable in the context of this $\mathrm{PhD}$, Table 5-2 sums up the information presented during this section.

Table 5-2: Power measurement procedures comparison

\begin{tabular}{|c|c|c|c|}
\hline Procedure & Resource measured & Sampling rate & Resolution \\
\hline \multirow{2}{*}{ k1-power } & Core & $50 \mathrm{~S} / \mathrm{s}$ & $1 \mathrm{~W}$ \\
\cline { 2 - 4 } & IO0 & $50 \mathrm{~S} / \mathrm{s}$ & $100 \mathrm{~mW}$ \\
\hline Oscilloscope & Core & $40 \mathrm{GS} / \mathrm{s}$ & $1.8 \mathrm{~W}$ \\
\hline Multimeter & Core & $1 \mathrm{~S} / \mathrm{s}$ & $18 \mu \mathrm{W}$ \\
\hline
\end{tabular}

To make the decision, it should be highlighted that, as the energy consumption model of this $\mathrm{PhD}$ will be built through $\mu$ benchmarking, the experiments can be developed to execute repetitive tasks during a long time. As a result, the sampling rate is not a constraint, but high resolution measurements are required to detect small changes associated to, for example, communication among resources or the computation of a single $\mathrm{PE}$. Consequently, during these tests, the Core power consumption will be measured with the 344450A multimeter, as it provides the highest power resolution, while the IOD DDR power consumption will be characterized using $k 1$-power, considering that it is the only instrument that can retrieve this value. Finally, it should be noted that, although $\mathrm{IO} 1$ could be intuitively considered equivalent to $\mathrm{IO} 0$, since its power cannot be measured, it has been decided to remove this resource from the model to avoid possible inaccuracies. 


\subsection{PAPI MPPA support}

Following the idea of giving access to low-level hardware performance information explained in Subsection 2.4.1 - Kalray S.A. has also included PMCs on its platforms. Specifically, the MPPA-256-N includes a total amount of 4 PMC 3 per core (i.e., 4 PMCs for each of the 256 cores within the CCs plus 4 per each of the 8 cores existing in the $\mathrm{IO}$ ). It should be noted that these counters are implemented as 32-bit counters, so their maximum value is $2^{32}-1(4,294,967,295)$. Regarding the information to gather, these PMC 3 can count the occurrence of 14 different events. Table 5-3 gathers information of these events, including a brief description of its occurrence meaning.

Table 5-3: PMC events available on the MPPA platform

\begin{tabular}{|c|c|}
\hline Event name & Short description \\
\hline _K1_CYCLE_COUNT & Clock cycles \\
\hline _K1_IHITS & Instruction cache hits \\
\hline _K1_IMISS & Instruction cache misses \\
\hline _K1_IMISS_STALLS & Stalling cycles after a instruction cache miss \\
\hline _K1_DHITS & Data cache hits \\
\hline _K1_DMISS & Data cache misses \\
\hline _K1_DMISS_STALLS & Stalling cycles after a data cache miss \\
\hline _K1_EXEC_BUNDLES & Bundles executed \\
\hline _K1_BR_TAKEN & Branches taken \\
\hline _K1_BR_TAKEN_STALLS & Stalls after taking a branch and re-fetching data \\
\hline _K1_RAW_STALLS & Stalls due to read after write \\
\hline _K1_ITLB_STALLS & Stalls due to instruction $\sqrt{\text { TLB }}$ refill \\
\hline _K1_DTLB_STALLS & Stalls due to data $\mu$ TLB refill \\
\hline _K1_STREAM_LOAD_STALLS & Stalls of streaming load buffer (uncached) \\
\hline
\end{tabular}

In order to ease the access to the performance information on the MPPA platform, during this PhD work, a new component has been added to PAPI library to support its use on both the $\mathrm{CC} s$ and the $\mathrm{IO}$. Additionally, it has been decided to develop it using Cluster-OS, a proprietary OS from Kalray, since it is supported in both CCs and IOs, while Linux OS is only supported on the IOs.

As the MPPA architecture was completely new from the point of view of PAPI library, its support has been done in 4 different stages. Specifically, porting this library has been performed taking into account that every CC (and IO) will need to initialize its own PAPI library, so, in the end, a distributed PAPI library is deployed:

1. PAPI lower layer: as a first step, a new component called $k 1$-mppa2 has been added within the options of PAPI. By doing so, the architectural-dependent layer of PAPI (its low level), which is in charge of managing the use of the PMCs, has been implemented. This component is able to access the 4 PMCs 
and to monitor the 14 different events. Additionally, this component has been developed relying on the library diagnostic . $h$, which is an internal library from Kalray that provides access to these PMCs. Moreover, the management of the PMC $s$ is performed in a core-wise mode, i.e., each core can only access the PMC 3 counting the events of that processor. Finally, as the Bostan version of the MPPA. does not support signaling from the PMCs, both multiplexing and overflowing functionalities have not been implemented.

2. PAPI upper layer: secondly, as the library will be based on the use of ClusterOS the operating system support of high level of PAPI needs to be adapted. The required support gathers providing OS information (memory constraints, working frequency, etc), specifying the thread lock mechanism and supporting accurate timing measurements (cycles and $\mu \mathrm{s}$ ). Finally, in order to unify the events from x86 architectures and the MPPA one, several preset events have been defined for the platform. These events are included in a file called k1papi_events.csv and summarized in Table 5-4.

Table 5-4: Preset PMC events available on the MPPA platform

\begin{tabular}{|c|c|c|}
\hline Event name & Short description & Event combination \\
\hline PAPI_TOT_CYC & Number of cycles & _K1_CYCLE_COUNT \\
\hline PAPI_L1_ICM & Instruction cache missed (level 1) & _K1_IMISS \\
\hline PAPI_L1_ICH & Instruction cache hits (level 1) & _K1_IHITS \\
\hline PAPI_L1_DCM & Data cache missed (level 1) & _K1_DMISS \\
\hline PAPI_L1_DCH & Data cache hits (level 1$)$ & _K1_DHITS \\
\hline PAPI_BR_TKN & Branches taken & _K1_BR_TAKEN \\
\hline PAPI_L1_ICA & Instruction cache accesses $(l e v e l ~ 1)$ & _K1_IMISS +_K1_IHITS \\
\hline PAPI_L1_DCA & Data cache accesses (level 1) & _K1_DMISS +_K1_DHITS \\
\hline PAPI_L1_TCM & Total cache misses (level 1) & _K1_IMISS +_K1_DMISS \\
\hline PAPI_L1_TCH & Instruction cache hits (level 1) & _K1_IHITS +_K1_DHITS \\
\hline PAPI_L1_TCA & Total cache accesses (level 1) & $\begin{array}{c}\text { K1_IMISS +_K1_IHITS + } \\
\text { +_K1_DMISS +_K1_DHITS }\end{array}$ \\
\hline
\end{tabular}

3. Installation: to ease the process of configuring, compiling and installing PAPI library on the MPPA both the configuration script and the Makefile have been modified so as to do it automatically. Specifically, as the compilation must be different for both the $\mathrm{IO}$ and the $\mathrm{CC}$, the procedure makes two compilations automatically and installs the generated static libraries.

4. Validation: finally, to test whether everything is working properly or not, some utils and tests provided by default in the PAPI repository have been adapted to evaluate the new implementation. On the one hand, these utils provide access to information of the available events in the platform. On the other hand, the tests that have been adapted are those that are not related to either multiplexing or overflowing, as those functionalities are not supported on the 
MPPA. Among the tests, there are 1) basic API usage (adding events, removing events, starting/stopping the count, etc); 2) multithread scenarios, 3) OpenMP testing and 4) utilities to characterize the monitoring performance itself.

Once the library implementation has been fulfilled and its behavior has been validated, its use needs to be characterized. In this line, PAPI|PMC basic management is composed of start, stop, read and write actions but, combining them, they provide a set of strategies to adapt the monitoring depending on the user requirements. As a summary, Table 5-5 gathers the clock cycles required to monitor from 1 to 4 events at the same time using each strategy. It should be noted that the displayed results are the average of 125,000 loop iterations. In this table, the first column represents the number of PMCs being monitored simultaneously: Start/Stop consists in starting the count, stopping it and reading the values; Read means that the PMCs are only read; Read with timestamp consists on reading the PMC 8 values together with a timestamp; Accumulate reads the values and adds them up to previous values without stopping the counting; finally, Reset only sets to zero the PMC count. As a result, in the worst-case -using the minimum working frequency of the MPPA (400MHz)- the maximum time required to access to this information, Start/Stop with 4 PMCs, is $3 \mu \mathrm{s}$.

Table 5-5: Required clock cycles to access the PMC

\begin{tabular}{|c|c|c|c|c|c|}
\hline$\#$ PMC $\$$ accessed & Start/Stop & Read & Read with timestamp & Accumulate & Reset \\
\hline 1 & 842 & 388 & 453 & 620 & 386 \\
\hline 2 & 968 & 468 & 488 & 727 & 439 \\
\hline 3 & 1,099 & 519 & 550 & 870 & 504 \\
\hline 4 & 1,231 & 561 & 609 & 999 & 592 \\
\hline
\end{tabular}

It should be highlighted that, during this implementation, the memory footprint of PAPI library for the MPPA has been drastically reduced. Specifically, for an x86 architecture, the standard PAPI library size is above $11.7 \mathrm{MB}$, while, in the case of the MPPA, it has been reduced to less than $200 \mathrm{kB}$. This is a critical factor when using the MPPA platform, as its CCs have a total memory of $2 \mathrm{MB}$ to be shared among $\mathrm{OS}$, system libraries, application code and application data.

\subsection{Resource-active contribution}

In this section, the energy consumption associated to the resource-active contribution is modeled. First, the structure of the architecture is analyzed to define a model. Then, specific $\mu$ benchmarks are developed to extract the power consumption associated to this contribution. Finally, the power consumption measured with these benchmarks is analyzed and the model of the resource-active contribution is extracted. 


\subsubsection{Model definition}

This partial model gathers the energy consumption associated to the activation of the platform resources, which are displayed in Figure 5-3. Specifically, this model will be used as a baseline power measurement, to which both computation and communication contributions are added up.

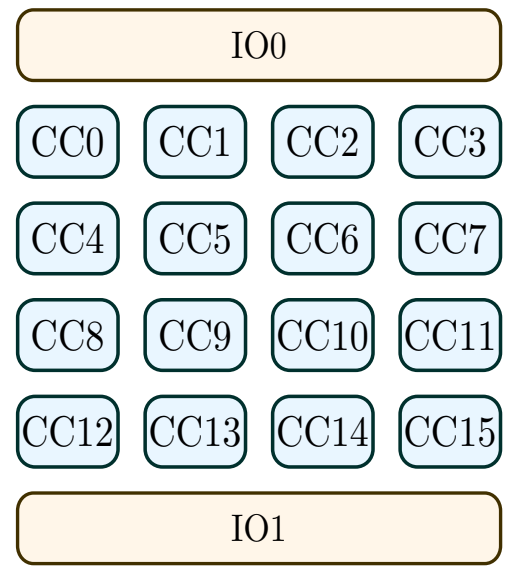

Figure 5-3: Platform resources (repeated from page 65

The energy consumption estimation will consider two values: the power consumed by the resource and the time it is operative. The former will be estimated based on the resources that are spawned, while the latter will be directly measured when the application is executed. Hence, Energ $y_{\text {resource-active will follow Equation 5-4. }}$.

$$
\text { Energy } y_{\text {resource-active }}=\text { Power }_{\text {resource-active }} * \text { Time }_{\text {application }}
$$

In this case, the value to estimate through the model will be the power the platform consumes just because the resources are switched on (Power resource-active $_{\text {). }}$. According to the MPPA structure, when one platform resource (IO) and/or CC 3 ) is spawned, its associated clock is turned on and, hence, the resource starts consuming. This means that the MPPA applies clock gating until one resource is explicitly spawned. This technique has been explained in Subsection 2.6.2.

Additionally, when developing an application on the MPPA, the developer can decide to use 3 working modes: 1) standalone $\mathrm{IO}$, 2) standalone $\mathrm{CC} s$ or 3) the whole platform (IO and $\mathrm{CC} s$ ). It should be reminded that, since the power consumption of the IO cannot be modeled, its usage is not considered in this part of the model. In this line, since each CC can also be spawned independently and their internal structure is identical, a linear model is proposed for this contribution.

It should be noted that the temperature of the platform may have an impact on this contribution, as it includes the power associated to the leakage and it varies with respect to the temperature. Consequently, an uncertainty factor $\left(U F_{t e m p}\right)$ per term 
in the polynomial has been introduced. The temperature will be measured using $\mathrm{kl}$ power, since it is the only instrument that can retrieve this information (Section 5.2).

As a result, 3 models are proposed depending on the aforementioned working modes: Equation 5-5 is associated to the standalone IO; Equation 5-6 represents the power consumption of the standalone $\mathrm{CC} \beta$, where the $\mathrm{DDR}$ power consumption is not included; and Equation 5-7 models the power consumption when all the available resources of the MPPA are employed.

$$
\begin{aligned}
& \text { Power }_{\text {resource-active-IO }}[\mathrm{W}]=a \pm U F_{\text {temp }} \\
& \text { Power }_{\text {resource-active-CC }}[\mathrm{W}]=\left(b \pm U F_{\text {temp }_{b}}\right) * N b \text { Clusters } \\
& \text { Power resource-active }[\mathrm{W}]=\left(a \pm U F_{\text {temp }_{a}}\right)+\left(b \pm U F_{\text {temp }}\right) * N b C l \text { usters }
\end{aligned}
$$

\subsubsection{Benchmark}

In order to measure the power associated to the activation of the different platform resources, a set of $\mu$ benchmarks is proposed. In this part, the resources will be gradually activated, holding each state (number of resources active) during 600 seconds. During this time, both the $\overline{D D R}$ memory and the Core power consumption -the one including the $\mathrm{CC} s$, the computational part of the $\mathrm{IO}$ and the NoC- will be measured to check possible impacts. In this line, 3 tests have been built:

1. IO activation: the $\amalg 0$ is activated and set to idle state.

2. CCs activation: the different $\overline{C C} s$ are spawned one by one without the $\mathrm{IO}$ being explicitly launched.

3. Platform activation: the $\mathrm{IO} 0$ is launched and, after that, each $\mathrm{CC}$ is spawned sequentially until all the platform resources are active and idling. Furthermore, in this test, one of the cores in the $\mathrm{IO} 0$ will work as the server for the asynchronous communications, which will be the methodology to communicate resources in real applications.

Finally, in order to analyze the impact of the temperature on the resource-active power consumption, a fourth experiment has been included. This experiment consists in computationally intensive slots interleaved with idle ones. By doing so, the platform is heated up and the impact of the cooling/heating phases of the architecture can be analyzed. In this case, every slot will be held during 300 seconds.

It should be noted that, as the MPPA does not support DVFS, the frequency to build the model has been fixed to $550 \mathrm{MHz}$, the maximum recommended. As a result, the benchmark will be composed of the $\mu$ benchmarks gathered in Table 5-6. 
Table 5-6: Resource-active $\mu$ benchmarks summary

\begin{tabular}{|c|c|c|}
\hline Name & Summary & Purpose \\
\hline IO activation & Turn on IOp & $\begin{array}{c}\text { Characterize IOP } \\
\text { baseline consumption }\end{array}$ \\
\hline CCs activation & Turn on the CCp one by one & $\begin{array}{c}\text { Characterize CCs } \\
\text { baseline consumption }\end{array}$ \\
\hline Platform activation & $\begin{array}{l}\text { Turn on IOp and } \\
\text { then the CC } \$ \text { one by one }\end{array}$ & $\begin{array}{c}\text { Characterize MPPA } \\
\text { baseline consumption }\end{array}$ \\
\hline Temperature & Interpolate stress and idle slots & $\begin{array}{l}\text { Characterize temperature impact } \\
\text { in the baseline consumption }\end{array}$ \\
\hline
\end{tabular}

\subsubsection{Model extraction}

In this part, the power consumption measurements obtained from the $\mu$ benchmarks will be used to adjust the coefficients of the model through linear regression, applying the methodology introduced in Subsection 2.5.3. To exemplify the obtained measurements, the test where the whole $\mathrm{MPPA}$ is used -Platform activation- is graphically analyzed and, then, the Temperature test is also evaluated.

In this regard, Figure 5-11 shows the measurements associated to Platform activation experiment, where a step chart is obtained for the Core power measurements. Each step is associated to the activation of one CC, which perfectly fits the proposed model for the resource-active contribution.

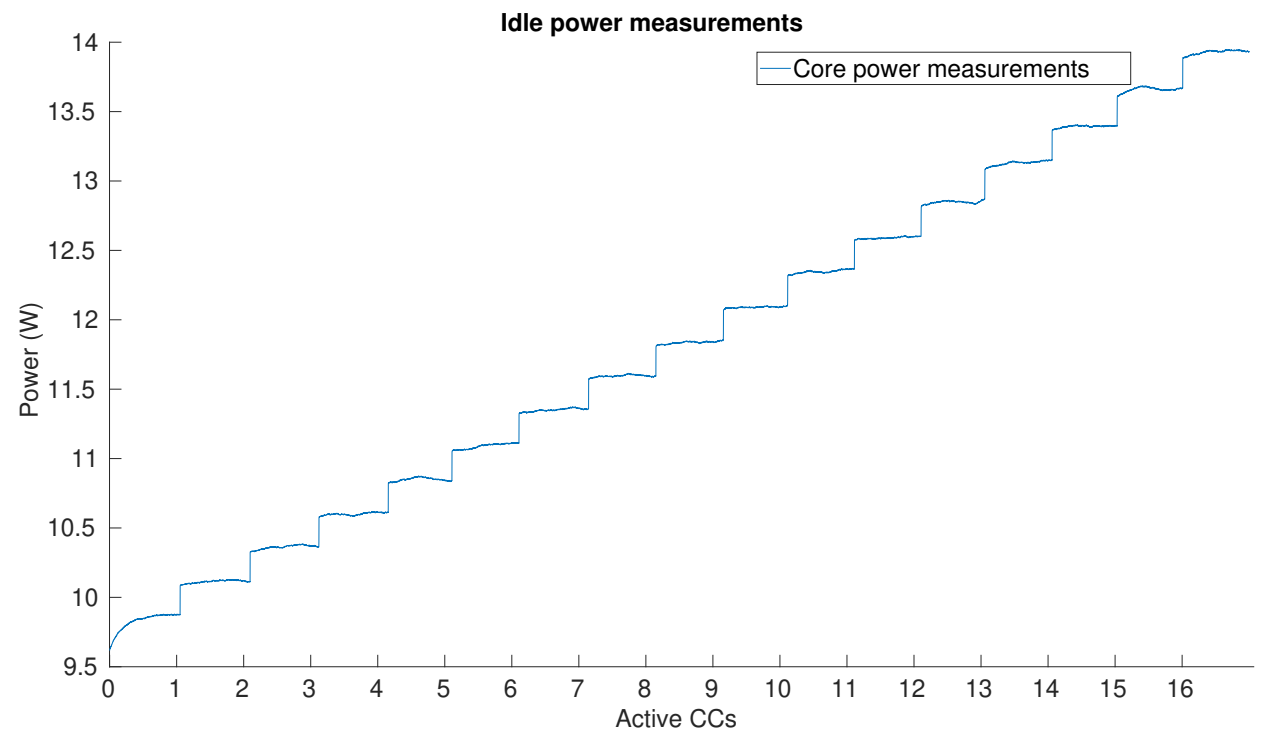

Figure 5-11: $\mathrm{IO}$ and $\mathrm{CC}$ s resource-active power consumption

Additionally, as mentioned before, the impact of the temperature on the resourceactive power consumption has to be analyzed. In Figure 5-12, computation stress time slots are interleaved with idle time slots. In this case, the Core power measurements 
are shown in blue, while the temperature is represented in red. As a result, it can be seen that there is a real impact on the power due to the temperature, which will be represented as a certain level of uncertainty in the estimation. For example, in the last experiment -highlighted with a green square in the figure-, the previous computational intensive slot has used $15 \overline{\mathrm{PE}} \beta$ and the temperature reached around $57^{\circ} \mathrm{C}$; hence, the impact of the cooling phase in the power consumption (blue line) of the idle slot can be seen, as it decreases along the whole idle slot.

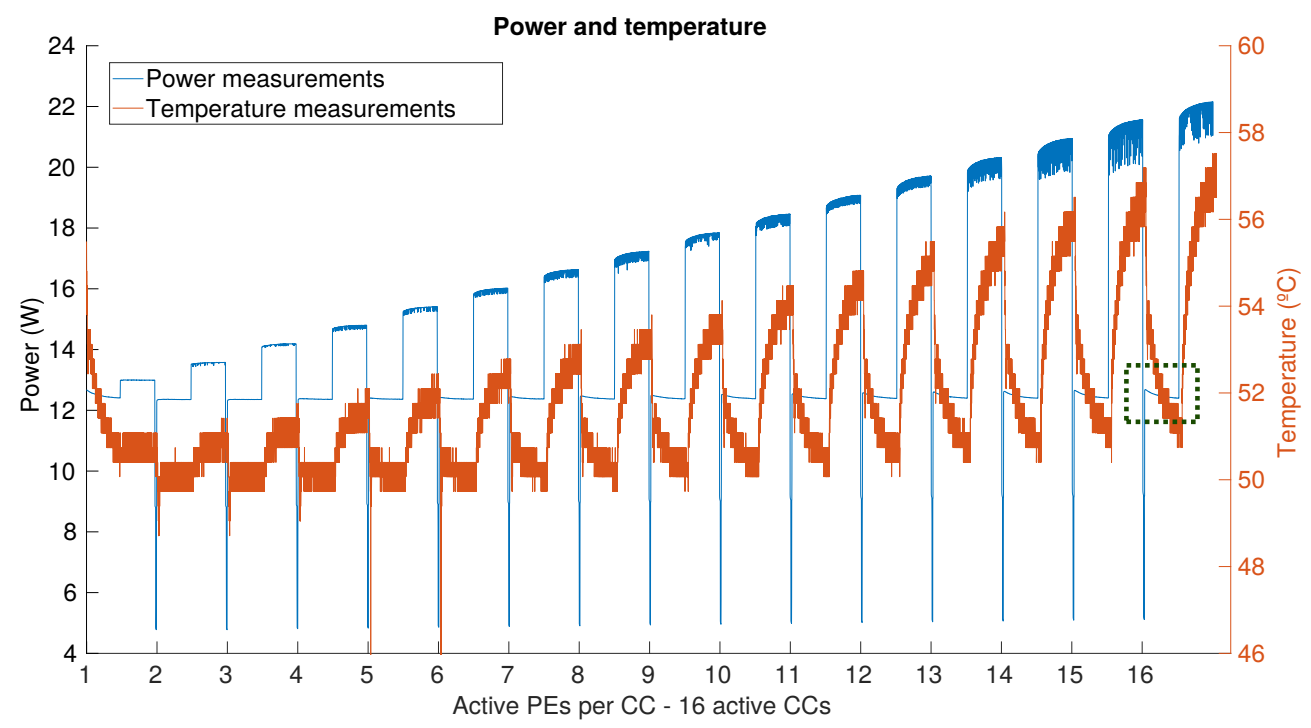

Figure 5-12: Temperature impact on resource-active power consumption

The same procedure has been repeated for the 3 working modes and, by doing so, each state has been characterized by a maximum, a minimum -obtained with the temperature test- and an average power consumption value -extracted from the basic $\mu$ benchmarks-. Consequently, there are 3 values for IO activation, 48 for CCs activation and 51 for Platform activation. Based on this information, the model coefficients are adjusted and 3 models are obtained. First, the model representing the standalone $\mathrm{IO}$ follows Equation 5-8. Secondly, the model associated to the standalone CC 3 mode is represented in Equation 5-9, Finally, the model representing the power consumed when all the platform resources are activated is the one shown in Equation 5-10.

It should be noted that, in Equation 5-10, the power consumed by the DDR memory used by the $\mathrm{IO}$ is included, which is why the power consumption is slightly above the one represented during the graphical analysis (Figure 5-11.

$$
\begin{gathered}
\text { Power }_{\text {resource-active-IO }}[\mathrm{W}]=7.736 \pm 0.038 \\
\text { Power }_{\text {resource-active-CC }}[\mathrm{W}]=(8.187 \pm 0.001)+(0.254 \pm 0.007) * \text { NbClusters } \\
\text { Power }_{\text {resource-active }}[\mathrm{W}]=(10.776 \pm 0.147)+(0.257 \pm 0.002) * \text { NbClusters }
\end{gathered}
$$


In order to quantitatively analyze these models, Table 5-7 gathers 1) the average relative error in \% (the lower the better), 2) the coefficient of determination $R^{2}$ (the higher the better) and 3) the Signal-to-noise ratio in $\mathrm{dB}(\mathrm{SNR}(\mathrm{dB}))$ (the higher the better). Additionally, the maximum percentage of uncertainty introduced by the temperature factor, UF, is also displayed. In this analysis, Power resource-active-IO model is only characterized by its uncertainty factor. Considering that in this model only the IO is used, the model coefficients have been directly linked with its power consumption measurements. For the other two models, the average relative errors are lower than the uncertainty factor percentage, which is aligned with the idea of introducing this parameter in first place. Finally, the high value of $R^{2}$ demonstrates that the models are able to properly represent the resource-active consumption data.

Table 5-7: Model evaluation for resource-active considering average relative error (in \%), coefficient of determination $R^{2}, \operatorname{SNR}(\mathrm{dB})$ and uncertainty due to temperature (\%)

\begin{tabular}{|c|c|c|c|c|}
\cline { 2 - 5 } \multicolumn{1}{c|}{} & Average relative error (\%) & $R^{2}$ & SNR(dB) & UF \\
\hline Power $_{\text {resource-active-IO }}$ & - & - & - & 0.49 \\
\hline Power $_{\text {resource-active-CC }}$ & 0.38 & 0.9979 & 45.65 & 0.44 \\
\hline Power $_{\text {resource-active }}$ & 0.82 & 0.9995 & 21.74 & 0.93 \\
\hline
\end{tabular}

\subsection{Communication contribution}

This section details the part of the model associated to the energy consumed when transmitting data among resources. Along this section, first, the architecture resources involved in the transmissions are analyzed to propose a model that properly represents this contribution. Later, a set of $\mu$ benchmarks is built to stress these resources in different ways. Finally, the measurements are correlated with the proposed model so as to extract the communication contribution model.

\subsubsection{Model definition}

Regarding the energy consumed when transmitting data, on the MPPA, there are 4 different cases depending on the source and destination of the transmission: $\mathrm{CC}, \mathrm{CC}$, CC. IO, IO, CC, IO, IO, which are represented in Figure 5-4, Specifically, this model is devoted to represent the amount of energy that is consumed during the whole communication process, namely reading data from the source memory, transmitting data through the NoC and writing data to the destination memory. This separation of concerns is represented in Equation 5-11. As a result, as the MPPA has a NUMA structure -where the memory is distributed among different hierarchies-, the power 
increment associated to both 1) the IODDR memory and 2) the CC 3 SMEM-included in Core-need to be taken into account. Finally, it should be mentioned that this model will be built using the asynchronous communications explained in [Hascoet'18].

$$
\text { Energ } y_{\text {comms }}[\mathrm{J}]=\text { Energ } y_{\text {source }}+\text { Energ } y_{\text {dest }}+\text { Energ } y_{N o C}
$$

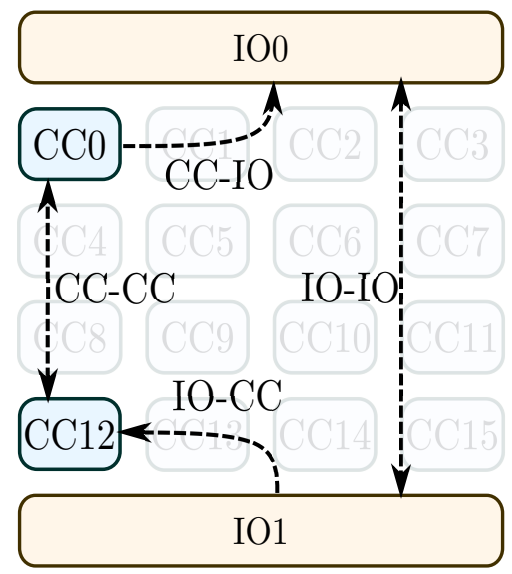

Figure 5-4: MPPA transmissions (repeated from page 65)

Analyzing in detail both Energ $y_{\text {source }}$ and Energ $y_{\text {dest }}$, two aspects need to be considered: the NUMA structure of the MPPA and the operation that is carried out. On the one hand, as introduced in Subsection 2.2.2, the IO has a DDR memory, while the CCs contain SMEM blocks. These two types of memory are different by construction, and using one or the other may have a different impact on the power consumed by the system. On the other hand, writing operations are usually more expensive than reading from memory in terms of both time and energy consumption. For these reasons, two cases are considered for reading (Energ $\left.y_{\text {source }}\right)$ and writing $\left(\right.$ Energ $\left.y_{\text {dest }}\right)$, depending on the memory that is being used, as shown in Equation 5-12. Finally, a parameter $T S$ has been added to represent the token size, since the communication among resources is based on a $\mathrm{NoC}$ and this value has a direct impact on the system bandwidth [Hascoet'18.

$$
\begin{aligned}
& \text { Energy }_{\text {sourc } \epsilon_{D D R}[\mathrm{~J}]=D D R} R_{0}+D D R_{R_{1}} * T S \\
& \text { Energ } y_{\text {sourc }}[\mathrm{SMEM}]=S M E M R_{0}+S M E M R_{1} * T S
\end{aligned}
$$

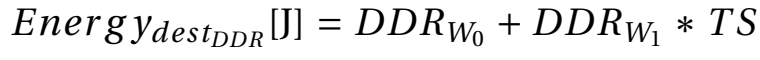

$$
\begin{aligned}
& \text { Energ } y_{\text {des }{ }_{\text {SMEM }}[\mathrm{J}]=S M E M}^{W_{0}}+S_{S M E M} W_{1} * T S
\end{aligned}
$$

In the case of Energ $y_{N o C}$, one of the aspects to be considered is the type of operation that is employed. As explained in Subsection 3.1.2, in asynchronous communications, either put or get operations can be used when communicating. Put operation directly sends the data from the source and writes on the destination memory. Get operation, in turn, is implemented as a reverse put, that is, a notification to request the transmission is sent, followed by the actual transmission. 
Analyzing the structure of the MPPANoC, shown in Figure 2-5, where each red line represents a link between DMAs, the data tokens will perform a different amount of hops depending on the source and destination of the transmission. On the MPPA. communication data paths are predefined to avoid possible deadlocks and they cannot be directly inferred from the NoC diagram itself. However, to illustrate this behavior, Table 5-8 gathers the number of hops required during inter-cluster communications. The first column, $S$, represents the source CC and the first row, $D$, the destination CC. As can be seen, this NoChas a symmetric configuration, i.e., sending data from CCp to CC15 requires the same hops than from CC15 to CCP.

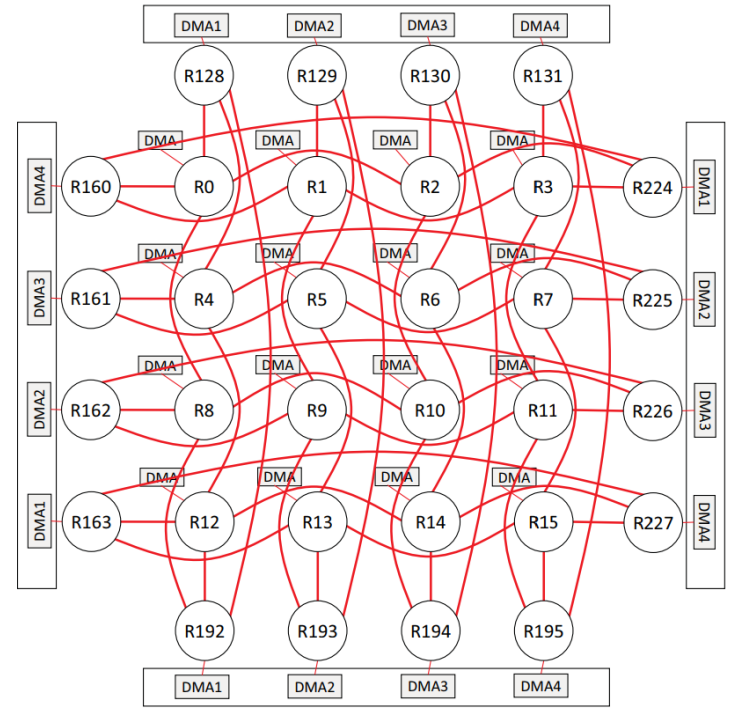

Figure 2-5 MPPA|NoC, Image extracted from [Maruyama'17] (repeated from page 15

As a result, the model associated to the energy consumed by the $\mathrm{NoC}$ is the one shown in Equation 5-13, where Hops represents the hops required to go from the source to the destination and Fct represents the impact of using get or put operations. Additionally, again, a parameter to consider the token size, $T S$, has been included.

$$
\operatorname{Energ}_{N o C}[\mathrm{~J}]=N O C_{0}+\left(N O C_{1} * H o p s * F c t * T S\right)
$$

Finally, the number of resources transmitting simultaneously has been included as a parameter to adjust the consumption in this equation to consider that, in simultaneous transmissions, the resources are shared. Specifically, $P_{T X s}$ is the parameter that modules the contribution of having $T X s$ simultaneous transmissions, which transforms Equation 5-11 into Equation 5-14. 
Table 5-8: Hops required on the MPPA NoC to communicate among CC. $S$ stands for source and $D$ means destination. The color is associated to the number of hops: green for 1-3, yellow for 4-6 and red for 7-10

\begin{tabular}{|c|c|c|c|c|c|c|c|c|c|c|c|c|c|c|c|c|}
\hline D & 0 & 1 & 2 & 3 & 4 & 5 & 6 & 7 & 8 & 9 & 10 & 11 & 12 & 13 & 14 & 15 \\
\hline 0 & 4 & 4 & 1 & 5 & 4 & 8 & 5 & 9 & 1 & 5 & 2 & 6 & 5 & 9 & 6 & 10 \\
\hline 1 & 4 & 4 & 3 & 1 & 8 & 4 & 7 & 5 & 5 & 1 & 4 & 2 & 9 & 5 & 8 & 6 \\
\hline 2 & 1 & 3 & 4 & 4 & 5 & 7 & 4 & 8 & 2 & 4 & 1 & 5 & 6 & 8 & 5 & 9 \\
\hline 3 & 5 & 1 & 4 & 4 & 9 & 5 & 8 & 4 & 6 & 2 & 5 & 1 & 10 & 6 & 9 & 5 \\
\hline 4 & 4 & 8 & 5 & 9 & 4 & 4 & 1 & 5 & 3 & 7 & 4 & 8 & 1 & 5 & 2 & 6 \\
\hline 5 & 8 & 4 & 7 & 5 & 4 & 4 & 3 & 1 & 7 & 3 & 6 & 4 & 5 & 1 & 4 & 2 \\
\hline 6 & 5 & 7 & 4 & 8 & 1 & 3 & 4 & 4 & 4 & 6 & 3 & 7 & 2 & 4 & 1 & 5 \\
\hline 7 & 9 & 5 & 8 & 4 & 5 & 1 & 4 & 4 & 8 & 4 & 7 & 3 & 9 & 2 & 5 & 1 \\
\hline 8 & 1 & 5 & 2 & 6 & 3 & 7 & 4 & 8 & 4 & 4 & 1 & 5 & 4 & 8 & 5 & 9 \\
\hline 9 & 5 & 1 & 4 & 2 & 7 & 3 & 6 & 4 & 4 & 4 & 3 & 1 & 8 & 4 & 7 & 5 \\
\hline 10 & 2 & 4 & 1 & 5 & 4 & 6 & 3 & 7 & 1 & 3 & 4 & 4 & 5 & 7 & 4 & 8 \\
\hline 11 & 6 & 2 & 5 & 1 & 8 & 4 & 7 & 3 & 5 & 1 & 4 & 4 & 9 & 5 & 8 & 4 \\
\hline 12 & 5 & 9 & 6 & 10 & 1 & 5 & 2 & 6 & 4 & 8 & 5 & 9 & 4 & 4 & 1 & 5 \\
\hline 13 & 9 & 5 & 8 & 6 & 5 & 1 & 4 & 2 & 8 & 4 & 7 & 5 & 4 & 4 & 3 & 1 \\
\hline 14 & 6 & 8 & 5 & 9 & 2 & 4 & 1 & 5 & 5 & 7 & 4 & 8 & 1 & 3 & 4 & 4 \\
\hline 15 & 10 & 6 & 9 & 5 & 6 & 2 & 5 & 1 & 9 & 5 & 8 & 4 & 5 & 1 & 4 & 4 \\
\hline
\end{tabular}

\subsubsection{Benchmark}

To stress the communications and to extract the models, the $\mu$ benchmarks proposed in this section will vary the different parameters explained during the model definition: token size, source and destination, type of operation (get/put), number of hops performed through the NoC and simultaneous transmissions.

Considering that the transmissions can involve the IO, the CCs or both, variations in the power consumption of DDR and SMEMs (Core) can be expected. Hence, these resources will be monitored during the execution of the experiments. Additionally, all the tests interleave transmission-intensive slots with idle slots of 300 seconds each, so as to measure the power consumption as the increment with regard to the idle state.

In first place, all the $\mu$ benchmarks developed in this case share the same variation in terms of token size and type of operation. Specifically, the token size will be modified from 4 bytes to $512 \mathrm{kB}$ by increasing the token size multiplying by 2 the previous size; that is, tokens from $2^{2}$ bytes to $2^{19}$ bytes will be employed increasing the exponent one by one. Additionally, as the platform communications are modeled based on the use of the asynchronous library, cases in which this communication is performed through get and put operations are included in all the benchmarks.

Regarding the hops, they have been modified differently depending on the source and destination of the transmissions: 
1. CCto IO data read from the SMEM and written to the DDR In this case, different clusters are considered to perform such operation. These clusters are selected considering the number of hops in the $\mathrm{NoC}$ ( 1 or 2 ) required to reach the $\mathrm{IO}$.

2. $\mathbf{C C}$ to $\mathbf{C C}$, data read from the SMEM and written to the SMEM, 5 different number of hops are used in this case: 1, 4, 5 and 10. Additionally, simultaneous transmissions between two clusters are tested.

3. IO to CC. data read from the DDR and written to the SMEM. The hops used are equivalent to the CC to IO ones.

4. I0 to I0, data read from the DDR and written to the DDR, No extra considerations have been included.

To sum up, as shown in Table 5-9, a total amount of $26 \mu$ benchmarks employing 18 token sizes each are considered, totaling 468 different working points.

Table 5-9: Communication $\mu$ benchmarks summary

\begin{tabular}{|c|c|c|c|c|c|}
\hline Name & $\begin{array}{c}\text { Memory } \\
\text { read }\end{array}$ & $\begin{array}{l}\text { Memory } \\
\text { write }\end{array}$ & Variations & $\begin{array}{l}\text { Total } \\
\text { tests }\end{array}$ & Purpose \\
\hline CC-IO & SMEM & DDR & $\begin{array}{c}\text { get / put } \\
\text { 4B to } 512 \mathrm{kB} \text { token size } \\
1 \text { or } 2 \text { hops } \\
\end{array}$ & 72 & $\begin{array}{c}\text { Characterize CC-to-IO } \\
\text { data transmissions }\end{array}$ \\
\hline CC. $\mathrm{CC}$ & SMEM & SMEM & $\begin{array}{c}\text { get/ put } \\
\text { 4B to } 512 \mathrm{kB} \text { token size } \\
1,4,5,10 \text { hops } \\
\text { Simultaneity }\end{array}$ & 288 & $\begin{array}{c}\text { Characterize CC-to-CC } \\
\text { data transmissions }\end{array}$ \\
\hline IO $\mathrm{CC}$ & DDR & SMEM & $\begin{array}{c}\text { get } / \text { put } \\
\text { 4B to } 512 \mathrm{kB} \text { token size } \\
1-2 \text { hops }\end{array}$ & 72 & $\begin{array}{c}\text { Characterize } \mathrm{IO} \text {-to-CC } \\
\text { data transmissions }\end{array}$ \\
\hline & DDR & DDR & $\begin{array}{c}\text { get / put } \\
4 \mathrm{~B} \text { to } 512 \mathrm{kB} \text { token size }\end{array}$ & 36 & $\begin{array}{c}\text { Characterize } \mathrm{IO} \text { - } 0 \text { - } \mathrm{IO} \\
\text { data transmissions }\end{array}$ \\
\hline
\end{tabular}

\subsubsection{Model extraction}

To extract the MPPA communication model, the experiments composing the benchmark are analyzed to assess the impact of each parameter. First, a study of the different parameters considered in Energ $y_{\mathrm{NoC}}$ is carried out, namely the influence of hops, the use of get/put operations, and the impact of simultaneous communications. Finally, Energ $y_{\text {source }}$ and Energ $y_{\text {dest }}$ are extracted by evaluating the experiments in which the DDR and the SMEM are read or written.

To analyze the aspects related to the Energ $y_{N o C}$, CC.CC experiments have been selected, as they present the largest variability. To analyze both the impact of hops and 
the type of operation being used, Figure 5-15 gathers the energy per transmission for each token size. Specifically, get and put operations are represented with continuous and discontinuous lines, respectively. Additionally, each number of hops is represented with a different color: black -1 hop-, red -4 hops-, blue -5 hops- and green -10 hops-. Furthermore, Figure 5-16 is a zoomed version reaching only data tokens until 16kB, where the energy consumption lines are intertwined along the chart. For this reason, no specific impact can be linked to the energy consumption but, to take advantage of these experiments, they will be averaged and reused during the rest of the section.

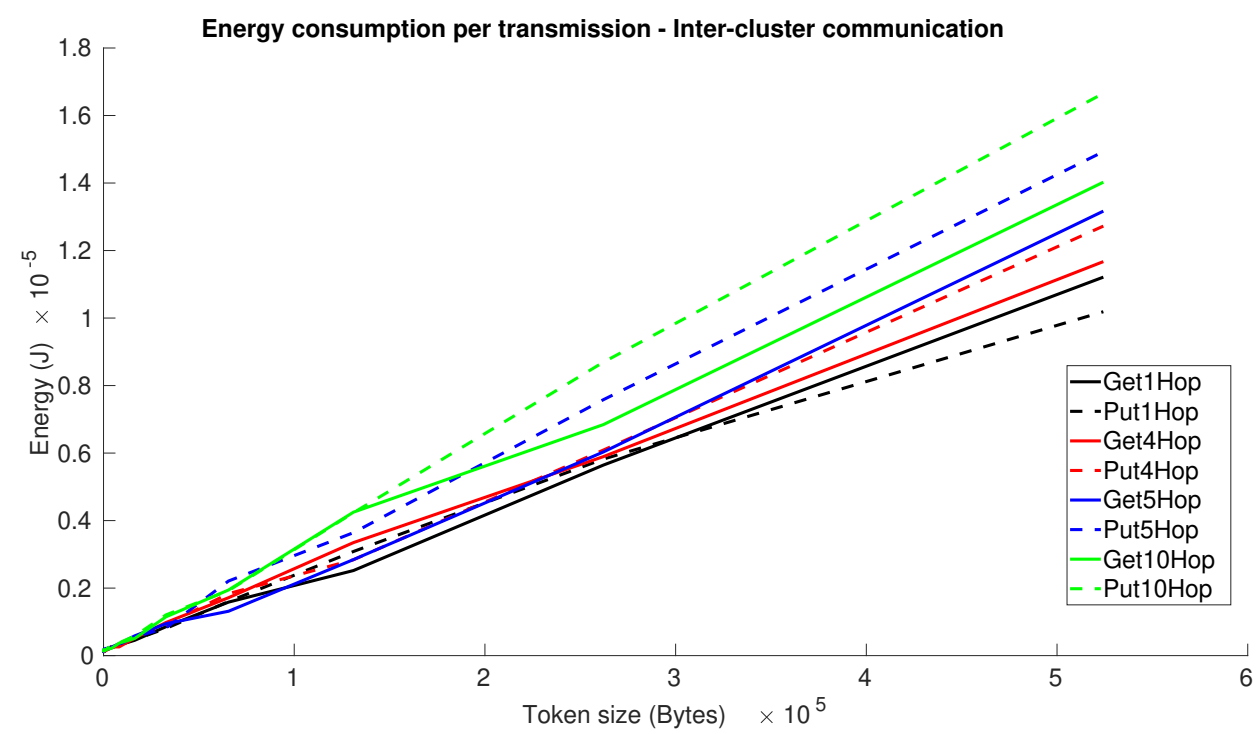

Figure 5-15: Energy consumption per transmission - CC-CC communication

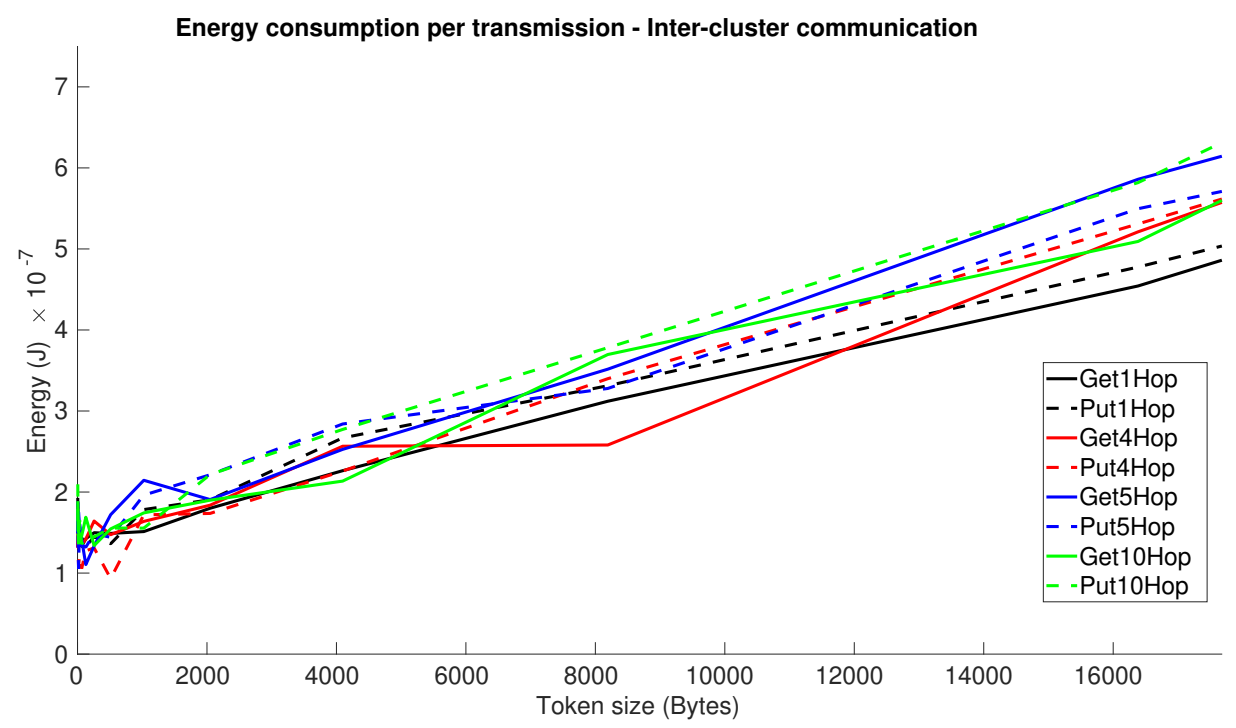

Figure 5-16: Energy consumption per transmission - CC-CC communication zoom (16kB) 
Regarding the impact of simultaneous communication strategies, Figure 5-17 gathers the average energy consumption per transmission for both cases. Specifically, considering the hops study, the results displayed are the average of all the previous cases for one transmission (in blue) and two simultaneous transmissions (in red). As can be seen, the energy per transmission is maintained for both cases. Consequently, the energy consumed per transmission can be considered independent from the number of clusters transmitting at the same time.

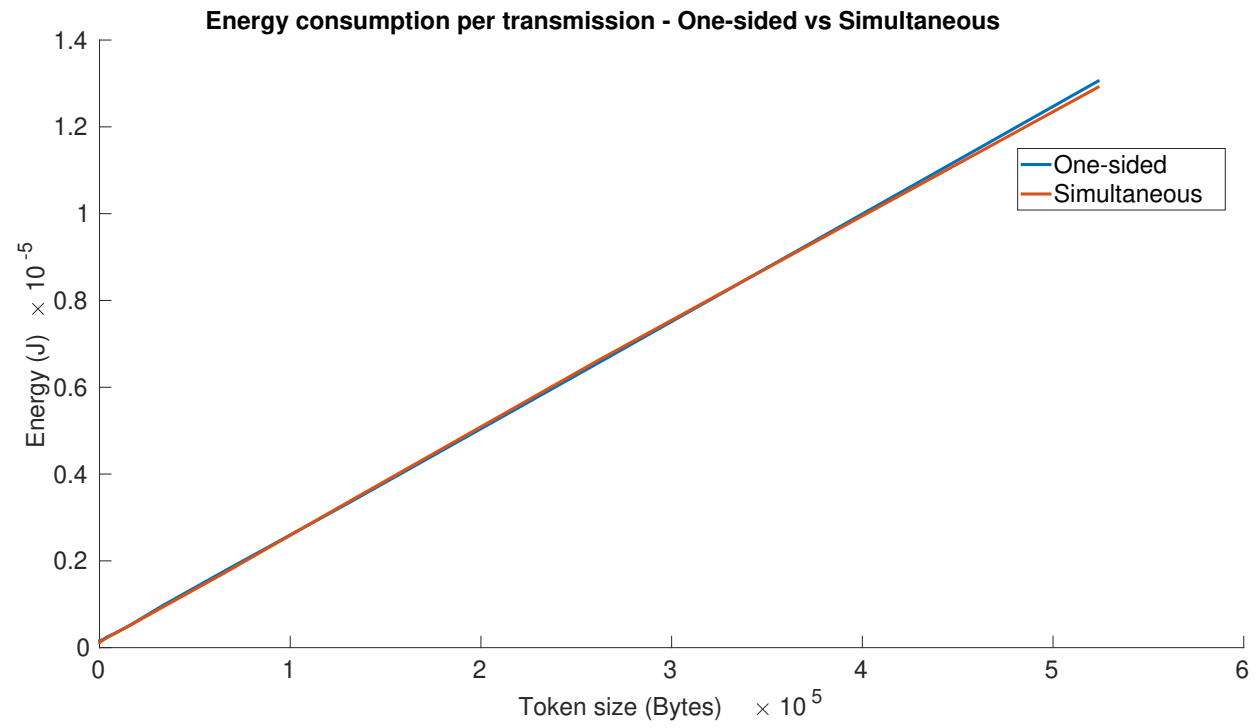

Figure 5-17: Energy consumption per transmission - One-sided vs simultaneous

Finally, the tests where the source and destination of the transmission vary are analyzed. First, the assumption of the differences in the power consumption obtained depending on the memory that is being used -DDR vs SMEM- and on whether it is being read/written -source vs destination- needs to be validated. In Figure 5-18, real power measurements for these 4 actions are displayed while varying the size of the token being transmitted. The measurements observed in this figure need to be analyzed by differences between the idle power consumption and the one associated to the communication itself, to isolate this contribution from the resource-active one -modeled in Section 5.4-. From this figure, some conclusions can be extracted:

- The DDR power consumption is higher than the one associated to the SMEM.

- The power consumption of writing in memory is higher than the one linked to reading from memories.

- Increments in the token size lead to increments in the power consumption of both memories and both actions.

To compare these 3 aspects (type of memory, read/write and token size), Figure 5-19 graphically represents a new study. This figure shows the energy 


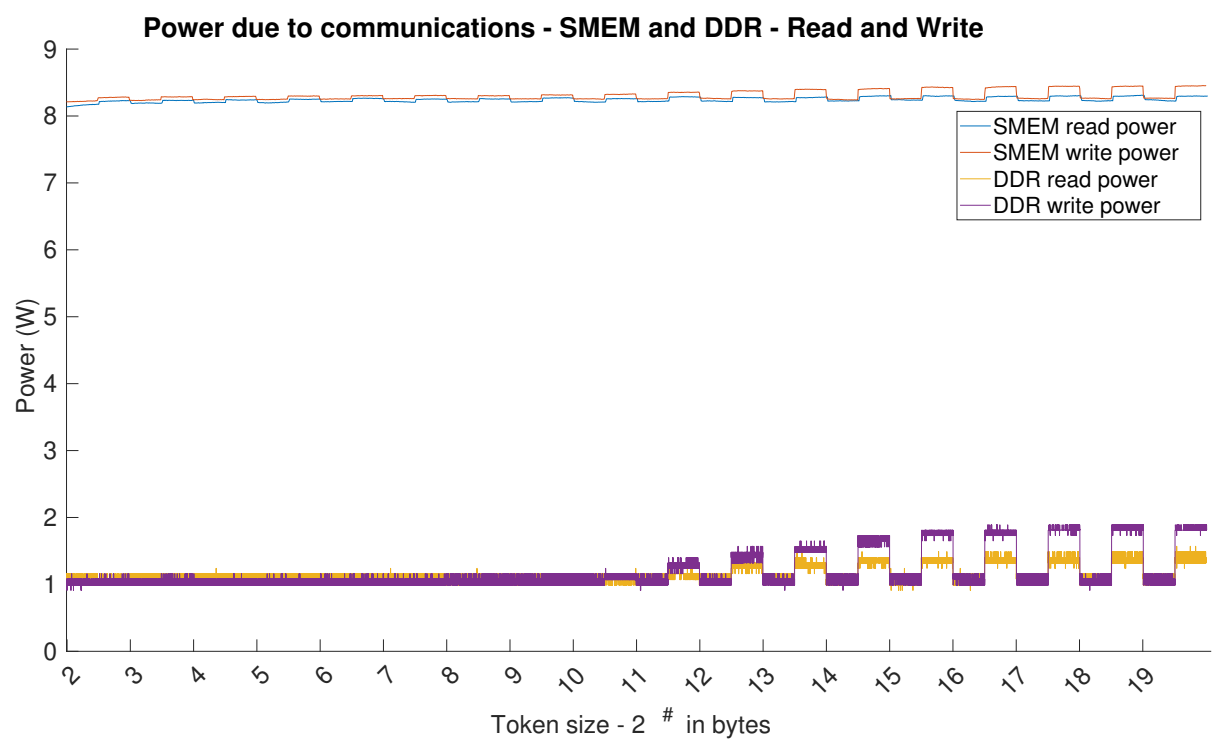

Figure 5-18: Power contribution comparison: SMEM vs DDR and read vs write. Idle and communication time slots are interleaved

consumption for every source and destination considering the average energy consumption of all the experiments belonging to each case. Specifically, CC-to-CC communication is represented in red, IO-to-CC is displayed in blue, CC-to-IO is shown in black and IO-to-IO is depicted in green.

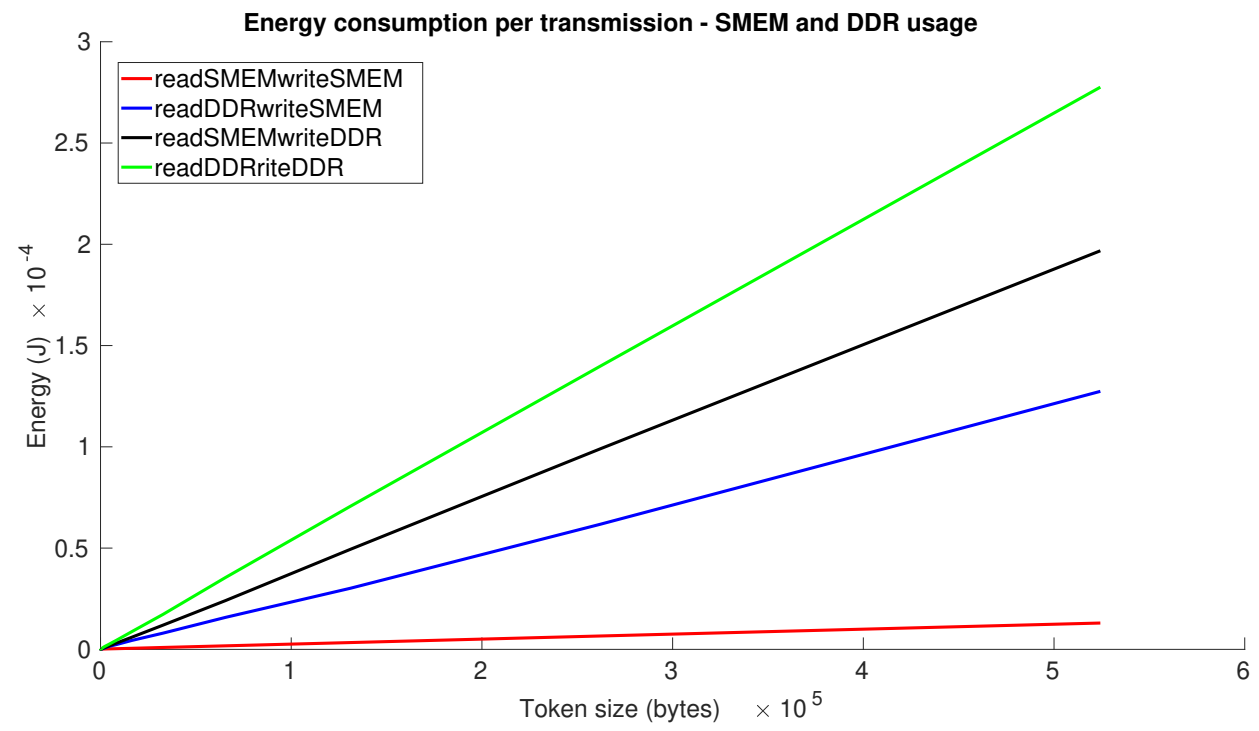

Figure 5-19: Energy consumption per transmission - SMEM and DDR usage

Analyzing these values, the differences between using SMEM or DDR during transmissions drastically overcome the contribution of the rest of the parameters. This implies that the model can be simplified so as to depend only on the source, 
the destination and the token size employed for the transmission, as reflected in Equation 5-15. In this case, the distinction between SMEM and DDR used as source (read) or destination (write) is embedded in a single formula.

$$
\begin{aligned}
& \text { Energy } \left.y_{\text {communication }}[\mathrm{J}]=S S M E M R_{R_{0}}+S M E M R_{1} * T S\right) * S M E M R_{\text {? }} \\
& \left.+\overline{D D D R}_{R_{0}}+\overline{D D R}_{R_{1}} * T S\right) * \overline{D D R}_{R_{?}} \\
& \left.+S_{S M E M} W_{0}+S M E M W_{1} * T S\right) * S M E M W_{3} \\
& \left.+D D R W_{0}+D D R W_{1} * T S\right) * D D R W_{?}
\end{aligned}
$$

where,

$$
\begin{aligned}
& \text { TS }=\text { Token Size (in bytes) } \\
& S M E M R_{?}=\text { Reading SMEM } \\
& \overline{D D R}_{W}=\text { Write } D D R
\end{aligned}
$$

$$
\begin{aligned}
& \operatorname{SMEM}_{R}=\operatorname{Read} \text { SMEM } \\
& \text { SMEM }_{W_{3}}=\text { Writing SMEM } \\
& \hline \overline{D D R} R_{?}=\text { Reading DDR? }
\end{aligned}
$$$$
\begin{aligned}
& \text { SMEM }_{W}=\text { Write SMEM } \\
& \hline \overline{D D R} \\
& \text { IDDR }_{W}=\text { Read } D D R \\
& W_{?}=\text { Writing } D D R
\end{aligned}
$$

In order to use this model, the user needs to know both the source (memory read) and the destination (memory write) of the data transmission, so as to set the parameters associated with ? symbol to 1 or 0 . That is, to include in the estimation only the terms corresponding to the memories involved in the data transmission.

After adjusting the coefficients of the model using linear regression, Equation 5-16 is obtained, which is graphically represented and compared to the real measurements in Figure 5-20. To complete this information, Figure 5-21 provides a zoom in of the part of the graph depicting data tokens below $4 \mathrm{kB}$. As can be seen, the estimations are close to the real values, but for the $D D R R_{R}-D D R{ }_{W}$ case, which is a type of communication rarely used in a real application. Nevertheless, the capacity of comparing SMEM and DDR consumptions when used to read/write data is obtained.

$$
\begin{aligned}
\text { Energ } y_{\text {communication }}[p J] & =(32647.43+1.25 * T S) *{ }_{S M E M} R_{?} \\
& +(41314.72+219.04 * T S) *{ }_{D D R} R_{?} \\
& +(85373.99+23.26 * T S) *{ }_{S M E M} W_{?} \\
& +(89096.37+373.01 * T S) * \overline{D D R} W_{?}
\end{aligned}
$$

In order to analyze the obtained model, Table 5-10 gathers 1) the average relative error in \% (the lower the better), 2) the Standard Deviation (STDEV) also in \% (the lower the better) and 3) the SNR(dB) (the higher the better) to understand how accurate the model is. Each row corresponds with a different type of transmission and each column provides a different indicator. With these results, it is worth pointing out that

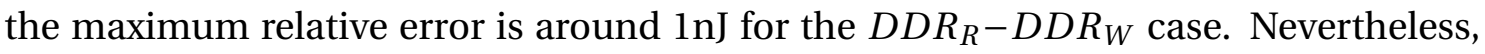
even though the average relative error of the $S M E M{ }_{R}-D D R{ }_{W}$ seems to be quite high (39.99\%), the usefulness and usability of the model will be evaluated together with the other two parts in Subsection 5.7.2. 


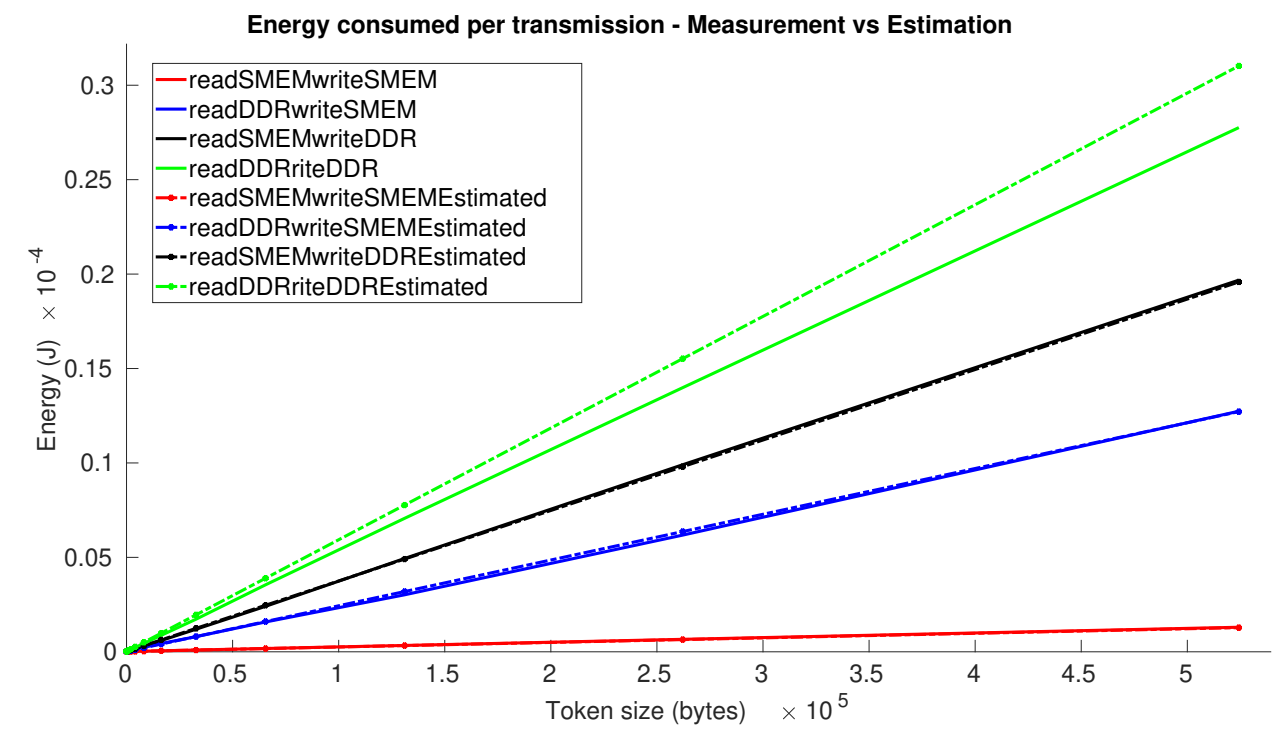

Figure 5-20: Energy consumption per transmission - Measurements vs estimation

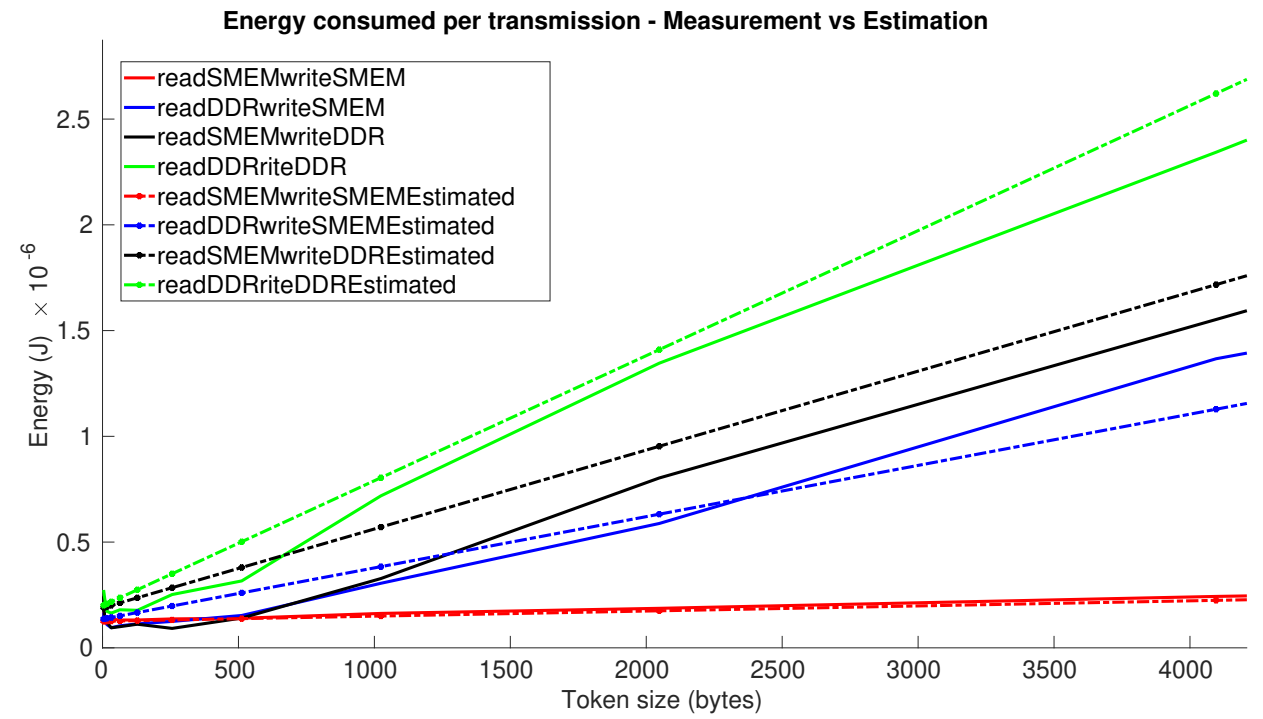

Figure 5-21: Power contribution comparison - Measurements vs estimation detail (4kB)

Table 5-10: Model evaluation for every type of communication considering average relative error (in \%), STDEV (in \%) and SNR(dB)

\begin{tabular}{|c|c|c|c|}
\hline & Average relative error (\%) & STDEV (\%) & SNR(dB) \\
\hline$S M E M_{R}-S M E M W$ & 2.68 & 4.85 & 47.25 \\
\hline$S M E M M_{R}-D D R{ }_{W}$ & 39.99 & 47.45 & 46.14 \\
\hline$D D R_{R}-S M E M M_{W}$ & 27.09 & 28.00 & 34.97 \\
\hline$D D R_{R}-D D R_{W}$ & 15.83 & 10.12 & 18.59 \\
\hline Global & 21.39 & 30.82 & 25.20 \\
\hline
\end{tabular}


After analyzing these values, it can be seen that this model provides enough information to discern the energy consumption contribution of the communications within an application. Additionally, with the information provided by the model, the developer can study the impact of deploying the application exploiting the inter-cluster communications or using a master-slave configuration applying the $\mathrm{IO}$ subsystem as a data provider. In this line, the former would be more efficient in terms of energy consumption than the latter.

\subsection{Computation contribution}

The last energy consumption contribution that will be modeled is the one associated to data processing tasks. As in the previous sections, first, the MPPA architecture will be analyzed to propose a model. After this, a set of $\mu$ benchmarks especially developed to stress computational resources is presented. Finally, the model is proposed based on the information extracted from the $\mu$ benchmarks.

\subsubsection{Model definition}

The model of the energy contribution associated to the computation will analyze how the energy is consumed in the computation resources of the MPPA when processing data. These resources, the CCs, shown in Figure 5-5. will be modeled from the point of view of the architecture to be independent of the type of processing being executed.

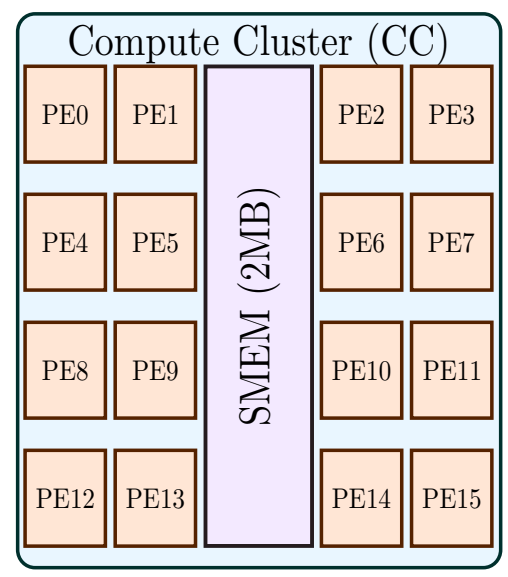

Figure 5-5: Compute Cluster (CC) (repeated from page 65

To maintain the application-independent approach, the proposed model will be based on PMC information, since it can be accessed from a high level of abstraction 
and represents detailed hardware utilization. To be precise, on the MPPA there is a total amount of 14 events that can be accessed through PAPI, as explained in Section 5.3.

In this regard, since there are only 4 PMC 3 available on the MPPA architecture, the information that can be retrieved simultaneously at runtime is limited to 4 events. As a consequence, the model proposed in this section will be composed of up to 4 events.

Additionally, as this part of the model aims at characterizing only the energy consumed when processing data, all the base power will be assumed to be included in the resource-active contribution. As a result, no constant term will be added in the computation polynomial model.

Finally, aiming at the idea of having a scalable model, Equation 5-17 should be also satisfied. By doing so, the model could be applied to only one $\mathrm{PE}$ (Energ $y_{P E}$ ) considering the hardware events that are obtained in that $\mathrm{PE}\left(E_{i}\right)$ and, additionally, combining all the event occurrences in all the platform $\operatorname{PE}\left(E_{i_{c c_{p e}}}\right)$, the same model could be applied to obtain the energy consumption of the whole MPPA (Energ $\left.y_{M P P A}\right)$. In order to satisfy this set of equations, the proposed model is linear, i.e., the events are not combined and a first degree polynomial is considered.

$$
\begin{gathered}
\forall N \in 1 \leq N \leq 4 \\
\text { Energ } y_{P E}=\sum_{i=1}^{i=N}\left(a_{i} * E_{i}\right) \\
\text { Energ } y_{C C}=\sum_{p e=0}^{p e=15}\left(\sum_{i=1}^{i=N}\left(a_{i_{p e}} * E_{i_{p e}}\right)\right) \equiv \sum_{i=1}^{i=N}\left(a_{i} * \sum_{p e=0}^{p e=15} E_{i_{p e}}\right) \\
\text { Energ } y_{M P P A}=\sum_{c c=0}^{c c=15}\left(\sum_{i=1}^{i=N}\left(a_{i_{c c}} * \sum_{p e=0}^{p e=15} E_{i_{c c p e}}\right)\right) \equiv \sum_{i=1}^{i=N}\left(a_{i} * \sum_{c c=0}^{c c=15}\left(\sum_{p e=0}^{p e=15} E_{i_{c c p e}}\right)\right)
\end{gathered}
$$

To sum up, an example of the MPPA computation model (Energ $y_{\text {comp }}$ ) is represented in Equation 5-18, where a total amount of 4 events ( $E_{0}, E_{1}, E_{2}$ and $E_{3}$ ) are employed and 4 coefficients $\left(C_{0}, C_{1}, C_{2}\right.$ and $\left.C_{3}\right)$ are associated to each event. This model is proposed to be independent of the number of PEs being considered, which means that it could be applied at 3 levels: $\mathrm{PE}$, $\mathrm{CC}$ s and MPPA.

$$
\text { Energy } y_{\text {comp }}[\mathrm{J}]=C_{0} * E_{0}+C_{1} * E_{1}+C_{2} * E_{2}+C_{3} * E_{3}
$$




\subsubsection{Benchmark}

The last set of $\mu$ benchmarks aims at stressing the platform resources when processing data in different ways. These benchmarks have been built in the form of kernels covering different types of computation together with different types of data: double, float and int. Specifically, 15 kernels compose this set:

1. Idle (Idle): the PE is put in an idle state. This kernel aims at setting as a baseline the resource-active consumption modeled during Section 5.4.

2. Branches (Bran): this test has been extracted from PAPI validation tests. It is a kernel written in assembly code that has 1,500,000 branches uniformly distributed among not-taken conditional branches, taken conditional branches and unconditional branches.

3. Instructions (Inst): this test has also been extracted from PAPI validation tests. It is a kernel written in assembly code that performs 1 million instructions.

4. Linear cache-double (LC-D): this test accesses the cache memory following a linear strategy. That is, it is iterating over an array (of double -64 bits per value) increasing the position to be accessed one by one.

5. Random cache-double (RC-D): this test accesses the cache memory following a random strategy. That is, it is iterating over an array (of double) changing the position to be accessed using the rand () function.

6. Operations - double (Op-D): this test carries out different operations. First, it performs a multiplication and an accumulation using double and, then, it stresses the cache memory by computing additions and deductions.

7. Matrix multiplication - double (MM-D): this test executes a naive matrix multiplication using double.

8. Linear cache - float (LC-F): same as 4 but using float (32 bits per value).

9. Random cache - float (RC-F): same as 5 but using float.

10. Operations - $\mathrm{float}(\mathbf{O p}-\mathrm{F})$ : same as 6 but using $\mathrm{float}$.

11. Matrix multiplication - float (MM-F): same as 7 but using $f$ loat.

12. Linear cache - int (LC-I): same as 4 but using int (32 bits per value).

13. Random cache - int (RC-I): same as 5 but using int.

14. Operations - int (Op-I): same as 6 but using int.

15. Matrix multiplication - int (MM-I): same as 7 but using int. 
Since the power consumption will be computed by differences with the baseline -the idle/resource-active consumption-, a single test in which all the kernels are executed sequentially has been built. In this test, for each kernel, the number of active PEs is increased one by one, maintaining each state during 90 seconds.

Additionally, to avoid possible distortions in the measurements due to the process of dumping PMC information into files, 5 different executions of the test have been performed. First, one test measuring the power consumption of Core (when modeling the $\mathrm{CC} s$ ) or $\mathrm{IO}$ DDR (when modeling IOP) will be launched. Then, 4 executions are required to retrieve the PMC information, since there are 14 available events and only 4 PMCs. In these 4 executions, the PAPI events will be sampled every second; hence, their correlation with the computational resources can be done either in terms of power or energy consumption.

Finally, it should be highlighted that, even if the $\mathrm{IO}$ is not usually employed to process data, it can be used for this purpose. Consequently, when applying these benchmarks to model the $\mathrm{CC} s$, the number of active $\mathrm{PE} s$ will be linearly increased from 1 to 256 , while, in the case of the $\mathrm{IO}$, the $\mathrm{PE} \beta$ will go from 1 to 3 . Summing up, Table 5-11 gathers the benchmarks built for this contribution.

Table 5-11: Computation $\mu$ benchmarks summary

\begin{tabular}{|l|c|c|c|c|}
\hline \multicolumn{2}{|c|}{ Name } & Kernels & PE & Purpose \\
\hline CC & computation stress & 15 & 256 & $\begin{array}{r}\text { Extract power and PAPI events for each type } \\
\text { of computation on the CC } \beta\end{array}$ \\
\hline IO & computation stress & 15 & 3 & $\begin{array}{r}\text { Extract power and PAPI events for each type } \\
\text { of computation on the IO }\end{array}$ \\
\hline
\end{tabular}

\subsubsection{Model extraction}

In this part of the model, the energy contribution due to data processing is characterized according to the total amount of PAPI events. This procedure is thoroughly analyzed when applied to the $\mathrm{CC} s$ and, then, it is used to model IOp.

Specifically, the first test that has been carried out aims at discerning if there is enough variability on the proposed kernels to properly represent different uses of the computational resources. To do so, in Figure 5-23, the energy measurements Figure 5-23a and the events generated for each kernel considered in this study Figure 5-23b to Figure 5-23o are represented.

In these graphs, each tick in the horizontal axis represents the starting point of each kernel. After each tick, the average energy consumption (Figure 5-23a) or the average event occurrences (Figure 5-23b to Figure 5-230) is displayed for 1 to 256 active $\mathrm{PE}$, being activated one by one, which is the reason behind the saw waveform in the figures. In this sense, it can be seen that the events differ considering not only the type of computation being performed, but also the type of data employed in the 

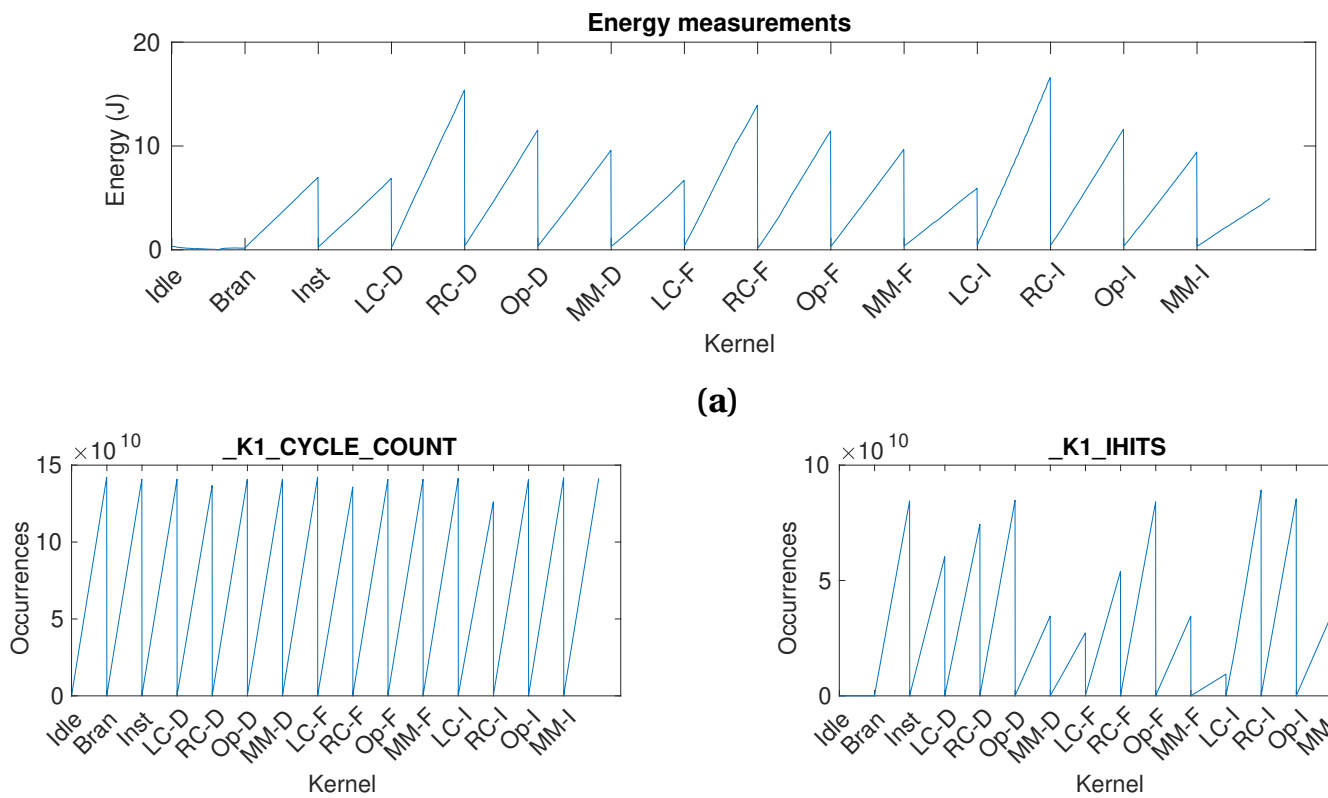

(a)

(b)

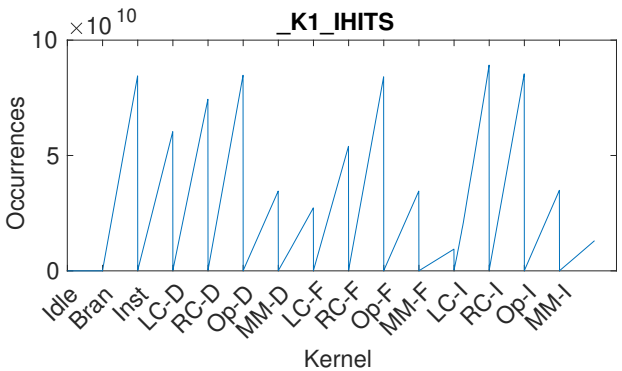

(c)
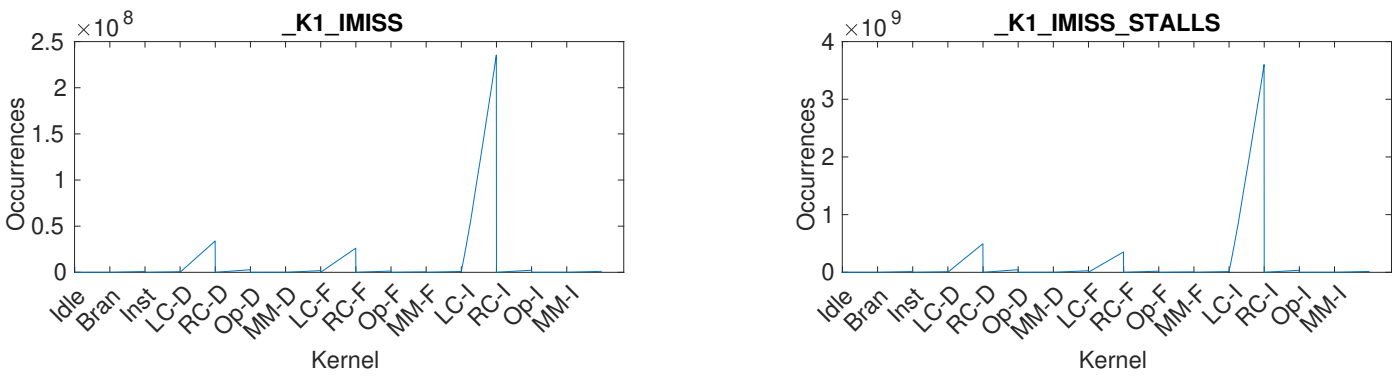

(d)

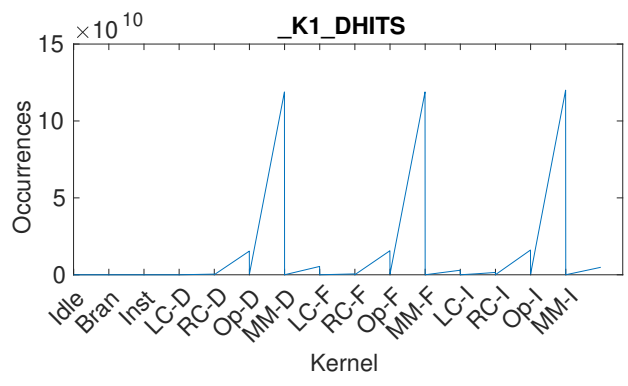

(e)

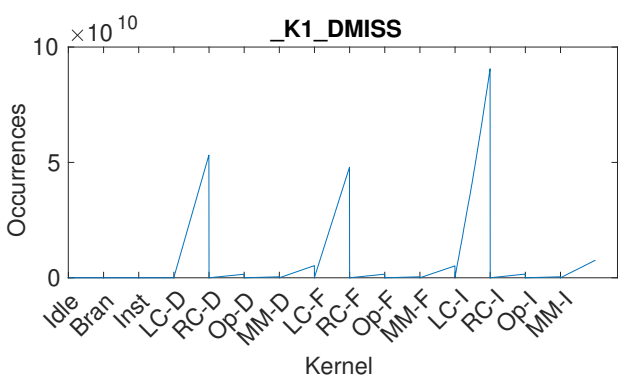

(g)

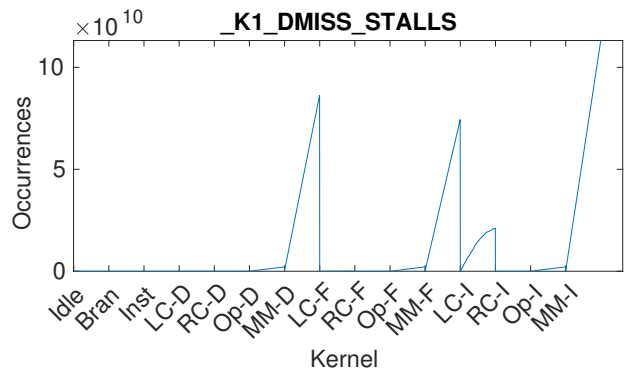

(h)

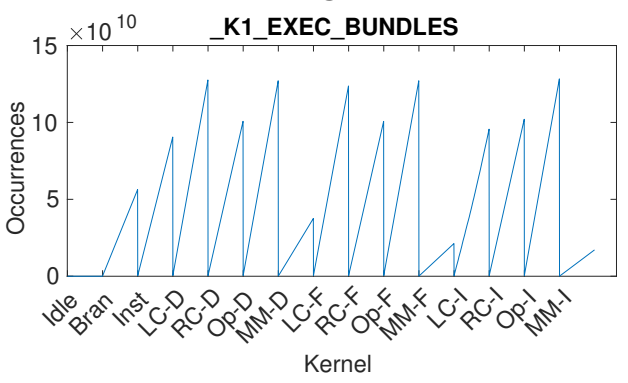

(i) 


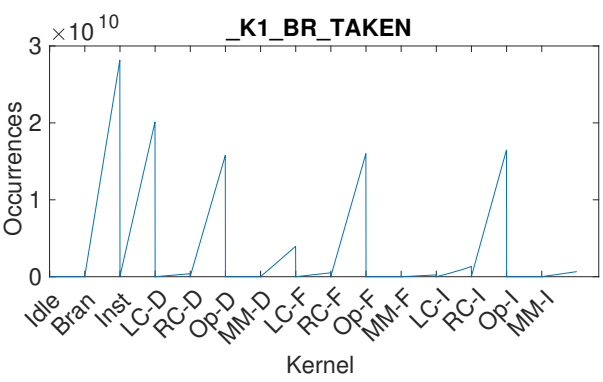

(j)

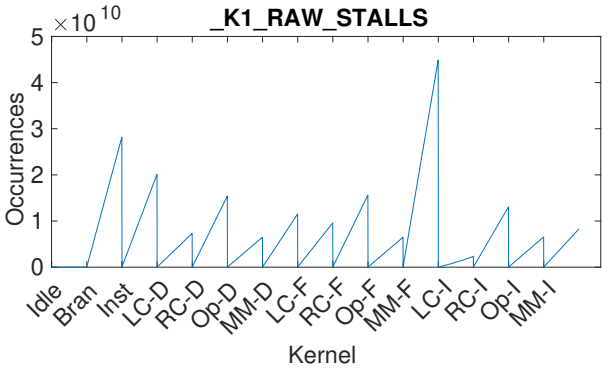

(1)

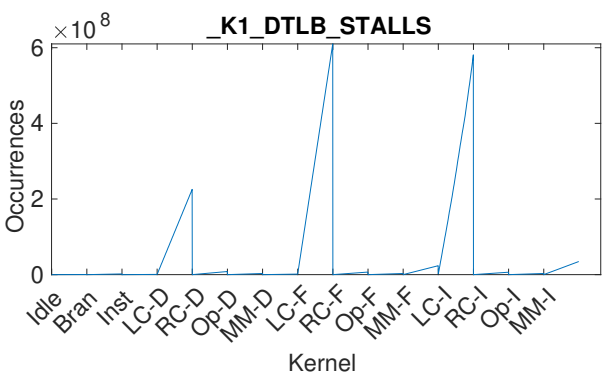

(n)

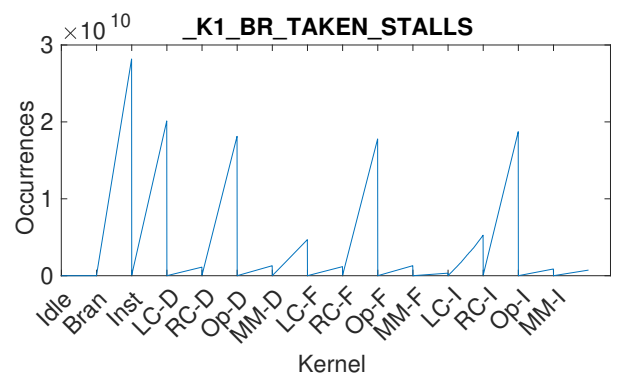

(k)

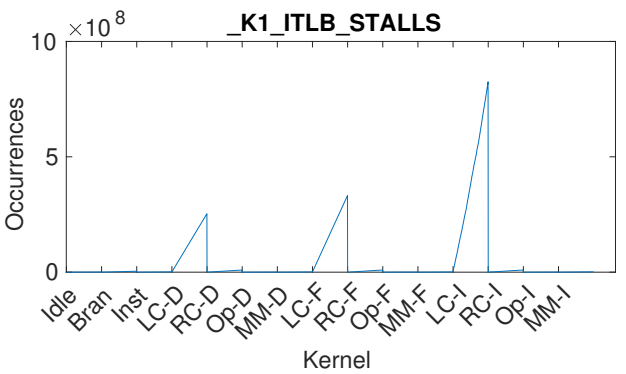

(m)

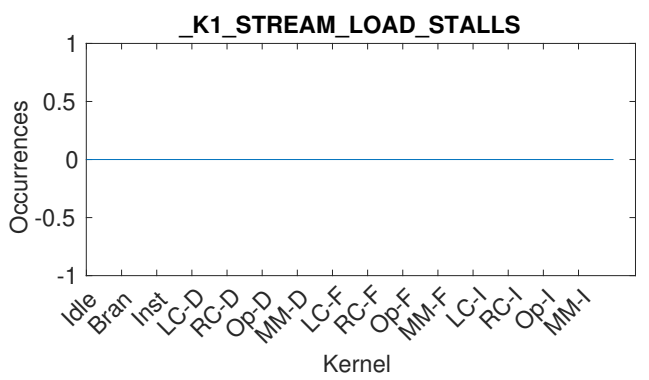

(o)

Figure 5-23: Energy and events obtained when executing the training set for computation. For the computation $\mu$ benchmark, (a) gathers the energy measurements, and from (b) to (o) event occurrences. Specifically, the events are (b) _K1_CYCLE_COUNT, (c) _K1_IHITS, (d) _K1_IMISS, (e)_K1_IMISS_STALLS, (f) _K1_DHITS, (g)_K1_DMISS, (h) _K1_DMISS_STALLS, (i) _K1_EXEC_BUNDLES, (j) _K1_BR_TAKEN, (k) _K1_BR_TAKEN_STALLS, (l) _K1_RAW_STALLS, (m) _K1_ITLB_STALLS, (n) _K1_DTLB_STALLS and (o) _K1_STREAM_LOAD_STALLS

kernel. Specifically, in kernels ending with $-D$, double data type is used; in the ones ending with $-F$, float data type is employed; finally, during the ones ending with $I$, int data type is characterized. However, there is almost no difference between the energy consumption of tests performing the same operation using different data types. Consequently, these kernels provide the dataset with different event combinations generating the same energy consumption.

In order to adjust the coefficients of the model, first, the dataset (composed of 3,840 working points) has been divided in training and test sets, with an $80-20 \%$ of the samples, respectively. Secondly, a series of subsets of samples used to adjust these coefficients are extracted from the training set using different criteria for each of them: 
- Total samples: to reduce the effect of over-fitting, models considering from $50 \%$ to $100 \%$ (in steps of $10 \%$ ) of the samples are employed.

- Uniformity: the samples are randomly selected either from the whole training dataset or taking the same number of samples from each kernel.

- Events: models considering from 1 to 4 events are considered. Additionally, all the combinations of events are tried.

To deal with the variability originated by the randomness in which the samples are selected, 20 models are generated for each criteria combination using a different subset of samples, which leads to the generation of a large set of candidate models -more than 50.000-. In order to decide which one is the best, they are tested against the aforementioned test set and characterized by its average relative error and the coefficient of determination $R^{2}$ [Glantz'90]. The first conclusion that has been extracted from this analysis is that models composed of 4 events, in general, have a higher $R^{2}$ than those using less events.

Figure 5-24 represents the models composed of 4 events based on their average relative error (vertical axis) and their $R^{2}$ (horizontal axis). To be precise, models in which the samples have been selected from the whole dataset are represented in blue, while the ones built using a homogeneous distribution among kernels are displayed in orange. In this figure, the best model -the one being at the bottom right-, belongs to the homogeneously distributed group, as it is the one with the lowest average relative error and the highest $R^{2}$. Nevertheless, the models are distributed all along the figure, as their estimations depend on the events that have been used to build them.

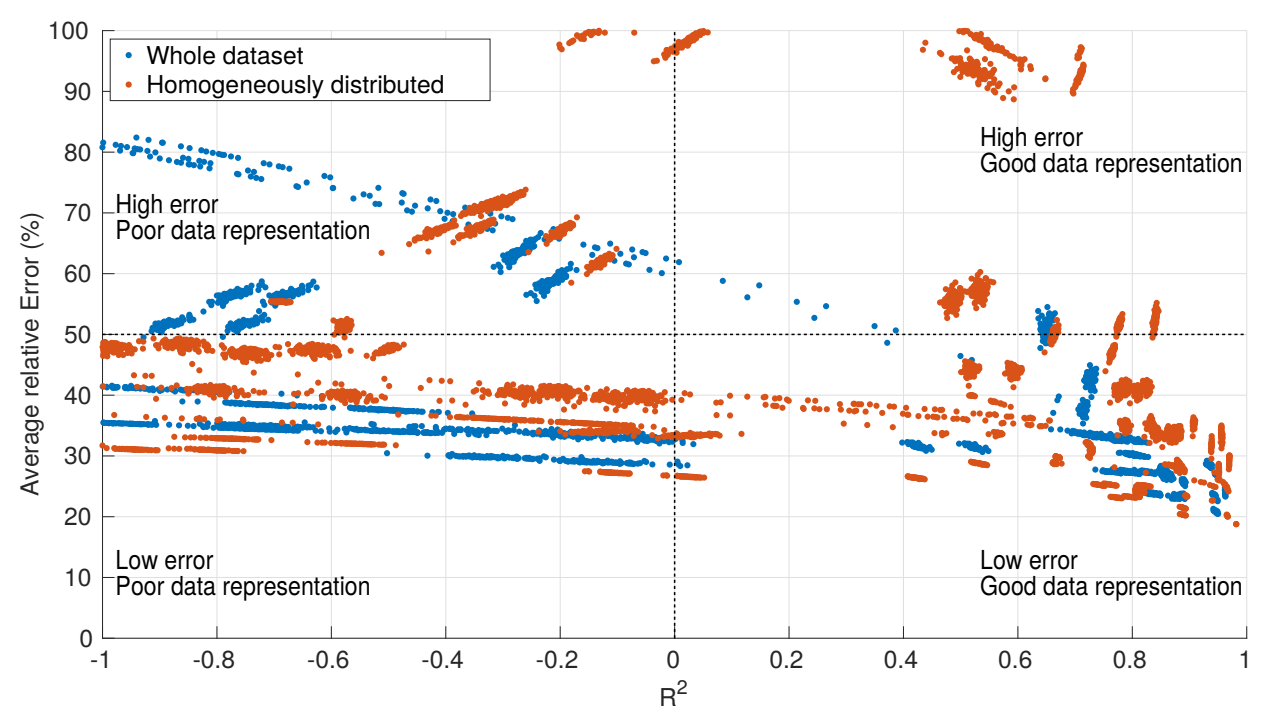

Figure 5-24: Computation contribution models analysis - Training samples uniformity

After analyzing all the candidate models, the one providing the best estimations is the one expressed in Equation 5-19, which considers the instruction cache hits 
(_K1_IHITS), the data cache misses (_K1_DMISS), the stalls due to data cache misses (_K1_DMISS_STALLS) and the bundles executed (_K1_EXEC_BUNDLES). In Figure 5-25, the estimations obtained with this model (in blue) are compared with the real measurements (in red). This model reaches a coefficient of determination $\left(R^{2}\right)$ of 0.983 and an average relative error of $18.35 \%$. The latter seems high, but it is mainly due to the difference between measurements and estimations of the Idle kernel, while the estimations in the other kernels are quite close to the real measurements.

$$
\begin{aligned}
& \text { Energ } y_{\text {comp }-C C}[\mathrm{pJ}]=59.311 * \_K 1 \_I H I T S+ \\
& 72.635 * \text { * } 1 \_D M I S S+ \\
& 31.127 * \text { * } 1 \text { 1_DMISS_STALLS+ } \\
& 64.5721 * \text { * } 1 \text { 1_EXEC_BUNDLES }
\end{aligned}
$$

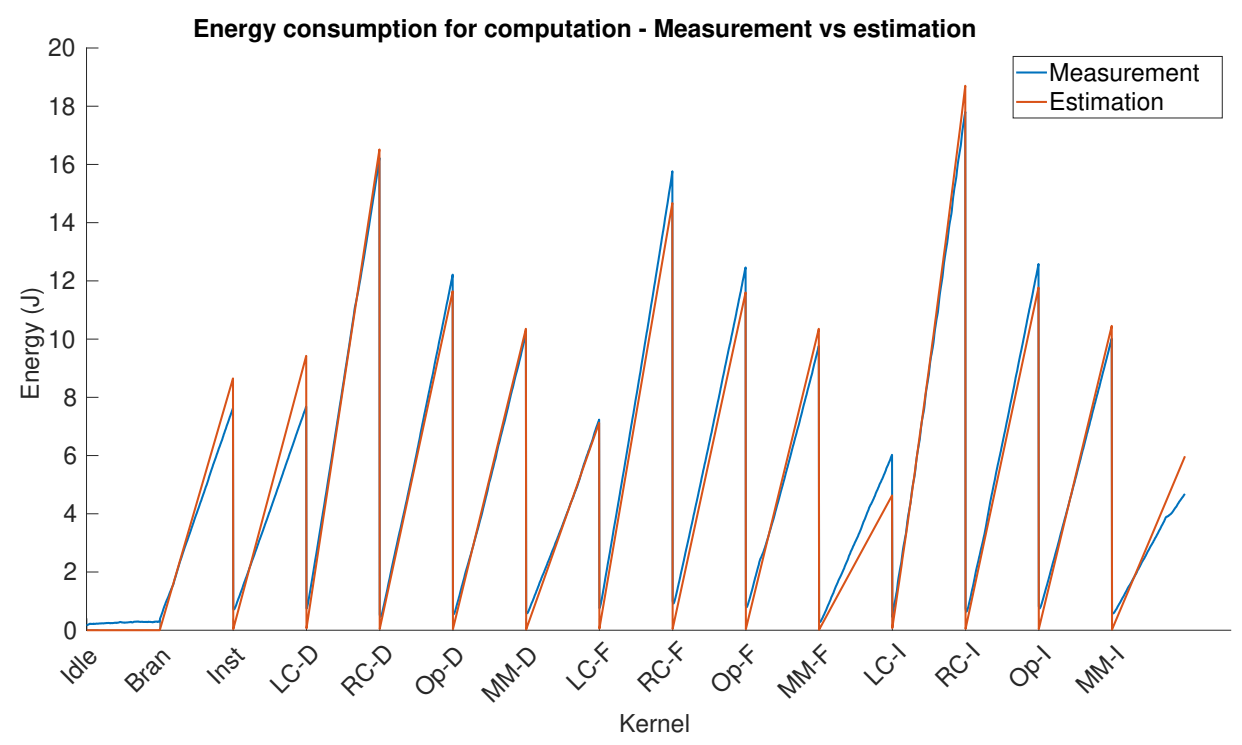

Figure 5-25: Energy measured vs energy estimated - Computation contribution

To analyze the impact of choosing different events, Figure 5-26 displays the models that have been built selecting the samples with a homogeneous distribution. To represent them, again, the average relative error and the coefficient of determination $\left(R^{2}\right)$ are used as the vertical and horizontal axes, respectively. The models are linked to a different color depending on how many of their events correspond to the ones used in the best model: 1) none of the events correspond (blue); 2) 1 out of 4 matches (orange); 3) 2 out of 4 are shared (yellow); 4) 3 out of 4 are the same (purple); and 5) the 4 coincide (green). From this analysis, several conclusions can be extracted:

- Models not sharing any of the events have always a negative $R^{2}$. This means that it is not possible to properly represent the energy consumption if none of the final events are considered. 
- In general, the more events are shared with the final model, the better estimations can be performed. For example, models sharing 3 events (purple) usually present a lower average relative error and a higher $R^{2}$ than those sharing 2 events (yellow).

- Models using the 4 final events (green) always appear in the bottom right part of the figure. This means that these events are the ones best representing how the energy is consumed in the platform.

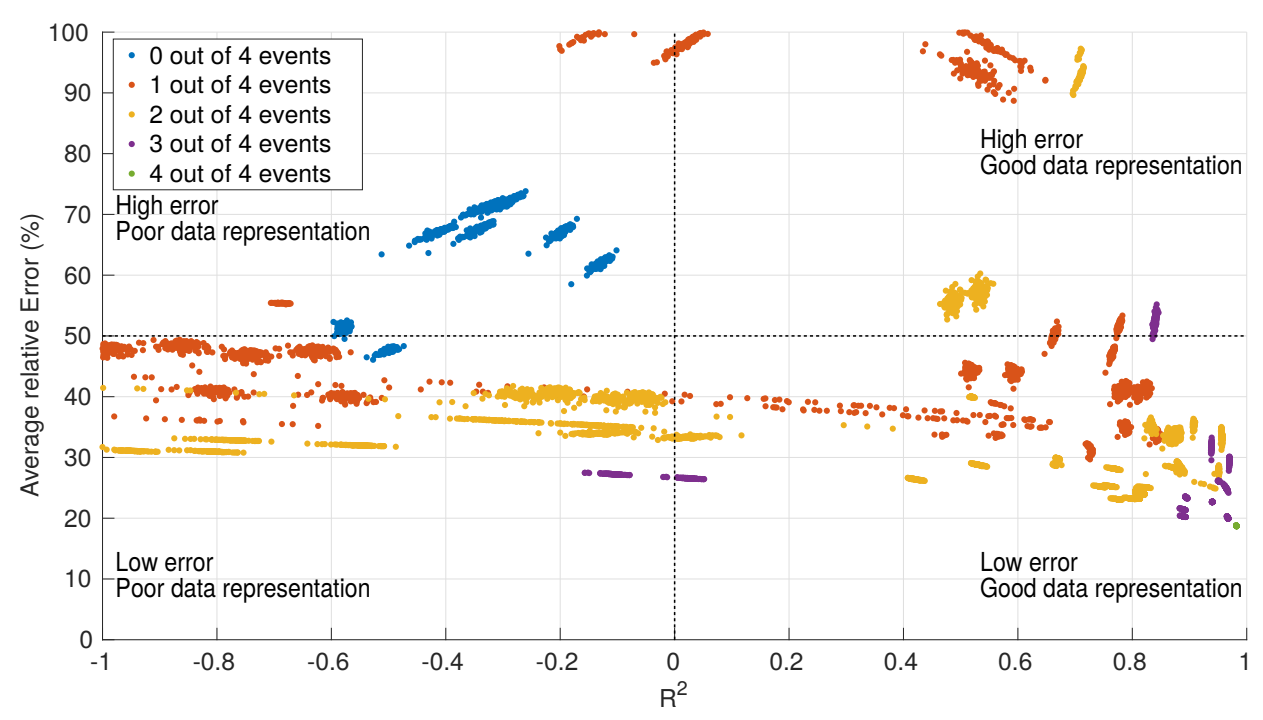

Figure 5-26: Computation contribution models analysis - Events selection

Doing a deep analysis of the relevance of each event within the selected model, two different studies are presented. The former analyzes the total amount of events of each type generated for each kernel, represented in Figure 5-27. The latter weighs those events using the parameters of the model, hence displaying the isolated contribution to the energy consumption of each event, as depicted in Figure 5-28. In both cases, the values have been represented using a bar plot, where each color represents a different event. Specifically, to simplify the analysis, only the experiment where 256 PEs are working in parallel is shown for each kernel. Focusing on the analysis of Figure 5-28, several conclusions can be extracted:

- The events contributing the most to the energy consumption are _K1_IHITS (instruction cache hits) and_K1_EXEC_BUNDLES (bundles executed).

- For all the kernels where the cache is linearly stressed ( $L C-D, L C-F$ and $L C-I$ ), _K1_DMISS gains importance in the estimation.

- For all the kernels associated to the matrix multiplication ( $M M-D, M M-F$ and $M M-I)$, _K1_DMISS_STALLS overcomes the rest of events in terms of contribution to the energy consumption. 


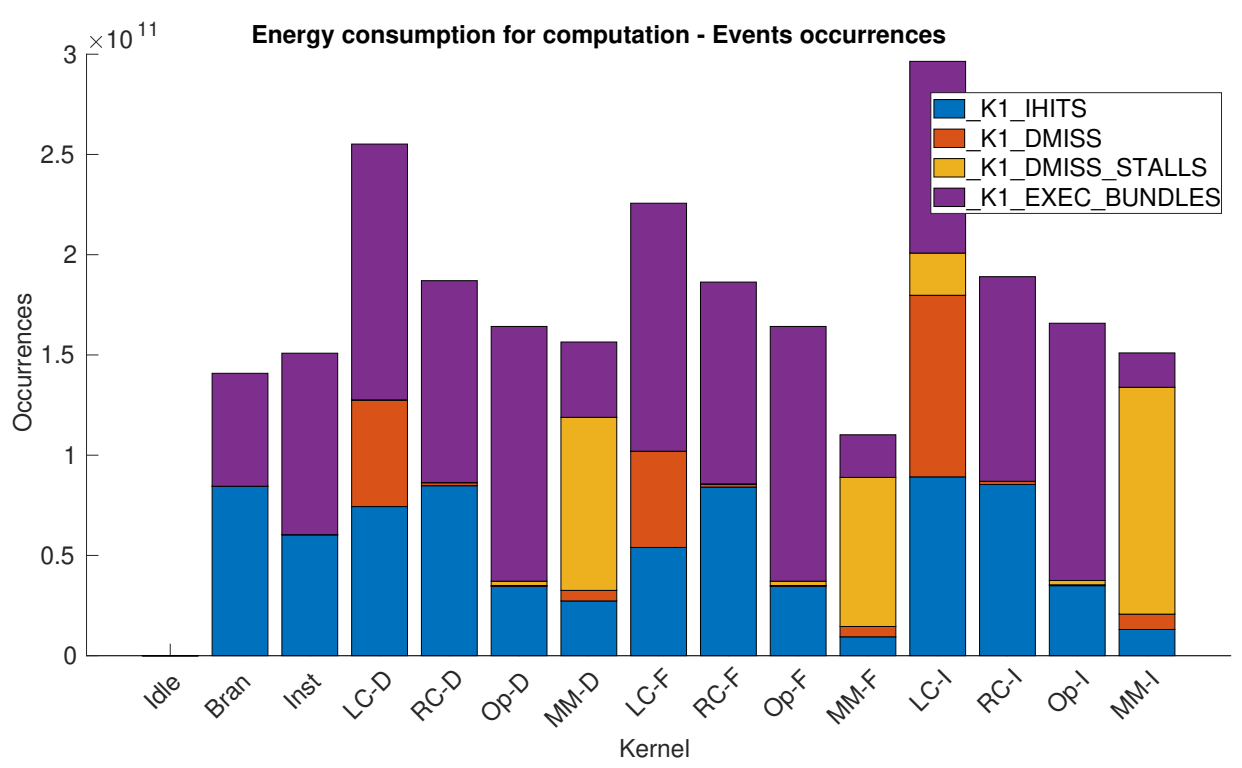

Figure 5-27: Energy consumption for computation - Events occurrences for each kernel in training set using the 256 $\mathrm{PE}$ available on the MPPA

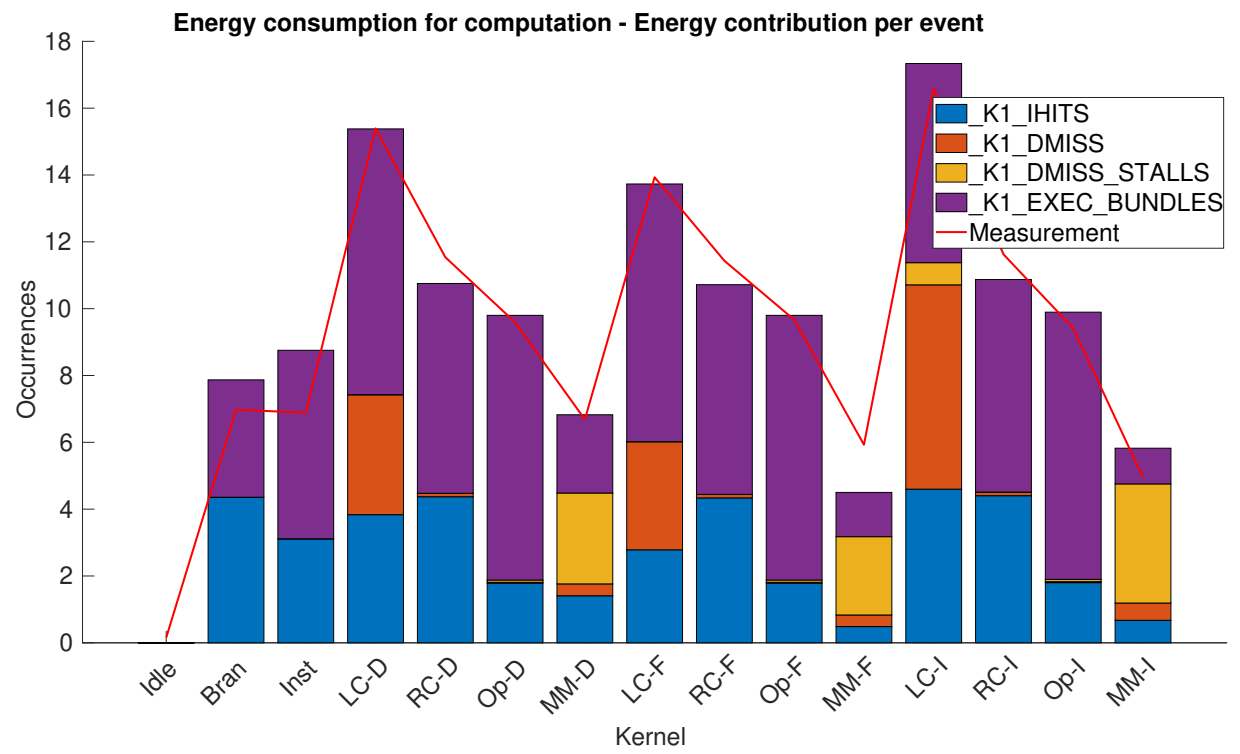

Figure 5-28: Energy consumption for computation - Energy contribution per event for each kernel in training set using the $256 \mathrm{PE}$ available on the MPPA 
The last study focuses on analyzing the impact of including several data types when training the model. Specifically, the selected model, hereafter all data types, is compared with 3 models which are trained using only double, float or int data types. Table 5-12 gathers this comparative, where the average relative error (\%), the $R^{2}$ coefficient and the $\mathrm{SNR}(\mathrm{dB})$ are considered. It should be noted that these values are computed against the whole test set, which includes kernels using the 3 data types.

Table 5-12: Model evaluation for computation using different training sets considering average relative error (in \%), the coefficient of determination $R^{2}$ and $\operatorname{SNR}(\mathrm{dB})$

\begin{tabular}{|c|c|c|c|}
\hline Model & Average relative error (\%) & $R^{2}$ & SNR(dB) \\
\hline All data types & 18.35 & 0.983 & 21.97 \\
\hline Only double & 27.42 & -0.049 & 4.24 \\
\hline Only float & 20.56 & 0.946 & 17.22 \\
\hline Only int & 19.91 & 0.984 & 20.74 \\
\hline
\end{tabular}

As can be seen, the all data types model is behaving better. Specifically, the coefficient of determination $\left(R^{2}\right)$ reaches a $98 \%$ in both all data types and only int model versions. Additionally, when comparing these two models, it can be observed that the all data types model has a lower average relative error and a better SNR(dB).

However, only double model obtains really poor results. These estimations are displayed in Figure 5-29 and, as can be seen, $L C-I$ experiment is not properly estimated. In this model, the selected events differ from the ones of the other models and_K1_DMISS event is not weighted in an equivalent way. Consequently, since this experiment is the one exploiting this resource the most, it can be concluded that the data cache memory consumption is not properly represented.

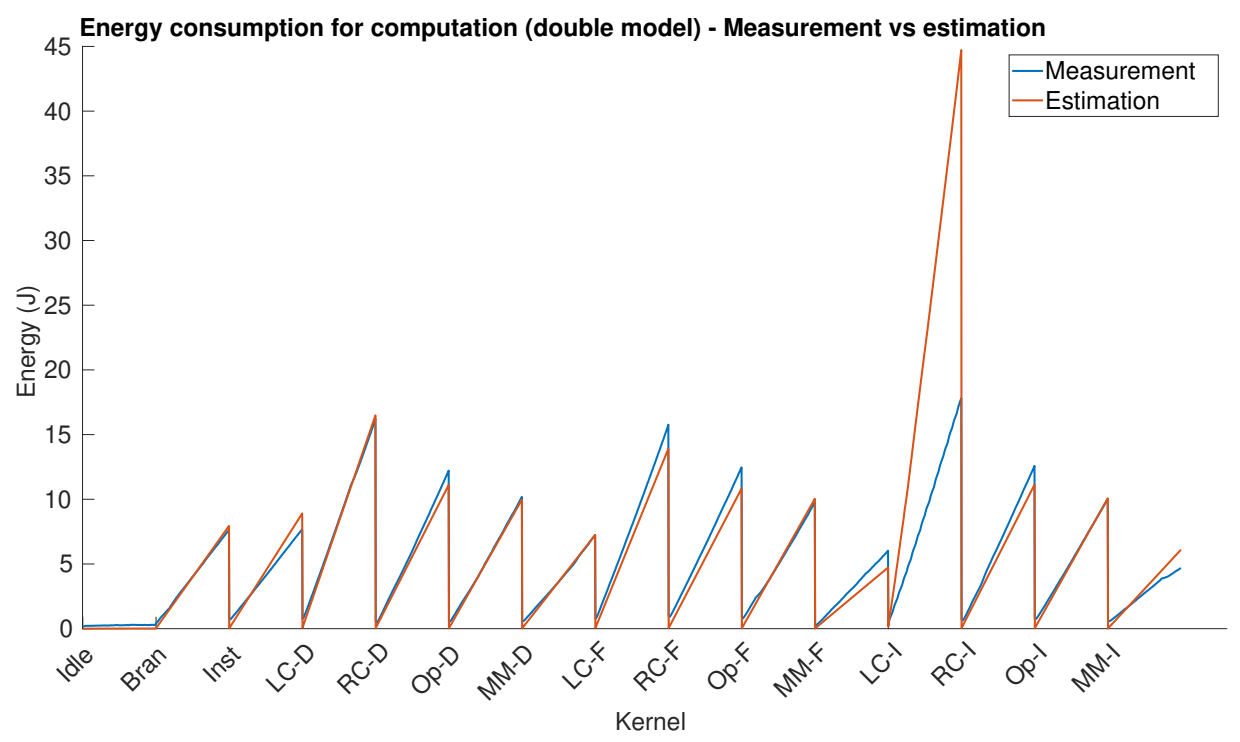

Figure 5-29: Energy measured vs energy estimated - Computation contribution using only double data types in the training phase 
Finally, although the $\mathrm{IO}$ is not usually employed to compute, it can be used for this purpose, so a computation model has been extracted for this resource. However, in comparison with the 3,840 samples -256 PEs by 15 kernels- composing the CCs dataset, in this experiment, only 45 samples -3 PEs by 15 kernels- are available. As a result, as can be seen in Table 5-13, the obtained model (Equation 5-20) has a lower $R^{2}$ and a higher average relative error than the ones obtained for the CC. Nevertheless, as displayed in Figure 5-30, the estimations are able to represent the differences among kernels, i.e., among types of computation.

It should be highlighted that, when comparing $\mathrm{CC}$ and $\mathrm{IO}$ computation models, both share the same events. However, the events related with the cache memory (_K1_IHITS, _K1_DMISS and_K1_DMISS_STALLS) have a coefficient 10 times higher in the $\mathrm{IO}$ case. This is aligned with the results obtained in the communication model Section 5.5-, where it has been observed that reading/writing from/to DDR consumes around 10 times more energy than reading/writing from/to SMEM.

Table 5-13: Model evaluation for computation in the $\mathrm{IO}$ considering average relative error (in $\%)$, the coefficient of determination $R^{2}$ and $\operatorname{SNR}(\mathrm{dB})$

\begin{tabular}{|l|c|c|c|}
\cline { 2 - 4 } \multicolumn{1}{c|}{} & Average relative error (\%) & $R^{2}$ & SNR(dB) \\
\hline Model all data types & 40.33 & 0.649 & 9.73 \\
\hline
\end{tabular}

$$
\begin{array}{r}
\text { Energ } y_{\text {comp }-I O[\mathrm{pJ}]=} 422.652 * \_K 1 \_I H I T S+ \\
787.864 * \text { } \& 1 \_D M I S S+ \\
391.801 \_K 1 \_D M I S S \_S T A L L S+ \\
28.794 * \_K 1 \_E X E C \_B U N D L E S
\end{array}
$$

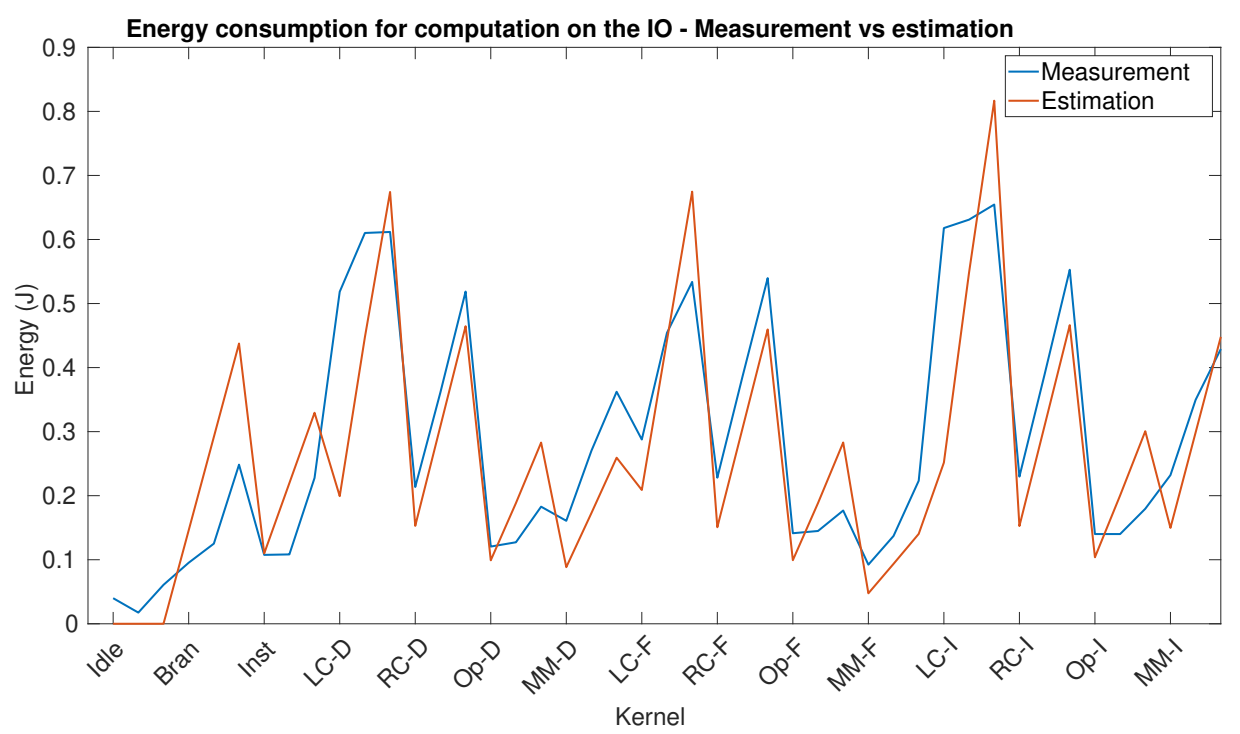

Figure 5-30: Energy measured vs energy estimated - Computation contribution on the $\mathrm{IO}$ 


\subsection{Platform model}

In this last section, the complete model of the architecture is summarized and evaluated. To do so, the validation process is carried out using GeMM application, which has not been included in any of the parts of the energy consumption model.

\subsubsection{MPPA-256-N energy consumption model}

In summary, Equation 5-21 shows the energy consumption model of the MPPA architecture developed in this chapter. In this model, the total energy consumption Energ $y_{\text {platform }}$ has been divided in 4 contributions:

- Resource-active contribution, Equation 5-22, weighted by the MPPA resources that are spawned and, thus, active.

- Communication contribution, Equation 5-23, which considers the source and destination memories and the transmitted token size.

- Computation contribution (CC), Equation 5-24, whose estimations are based on 4 PAPI events.

- Computation contribution (I0), Equation 5-24, where, if necessary, the estimations are also carried out considering 4 PAPI events.

$$
\begin{array}{r}
\text { Energ } y_{\text {platform }}[\mathrm{J}]=\text { Power }_{\text {resource-active }}[\mathrm{W}] * \text { ExecutionTimeApp }[\mathrm{s}]+ \\
\text { Energ } y_{\text {comms }}[\mathrm{pJ}] * 10^{-12}+ \\
\text { Energ }_{\text {comp }-C C}[\mathrm{pJ}] * 10^{-12}+ \\
\operatorname{Energ}_{\text {comp-IO }}[\mathrm{pJ}] * 10^{-12}
\end{array}
$$

Power $_{\text {resource-active }}[\mathrm{W}]=(10.776 \pm 0.147)+(0.257 \pm 0.002) *$ NbClusters

$$
\begin{aligned}
& \text { Energ } y_{\text {comms }}[p J]=(32647.43+1.25 * T S) * S M E M R_{R} \\
& +(41314.72+219.04 * T S) * D D R R_{R} \text { ? } \\
& +(85373.99+23.26 * T S) * S M E M W_{W_{2}} \\
& +(89096.37+373.01 * T S) * D D R W_{\text {? }}
\end{aligned}
$$

Energ $y_{\text {comp }-C C}[\mathrm{pJ}]=59.311 * \_K 1_{-}$IHITS+

$$
72.635 * \text { * K1_DMISS+ }
$$

$$
31.127 * \text { * } 1 \_D M I S S \_S T A L L S+
$$$$
64.5721 * \text { * } 1 \text { 1_EXEC_BUNDLES }
$$ 


$$
\begin{array}{r}
\text { Energ } y_{\text {comp }-I O[\mathrm{pJ}]=} 422.652 * \_K 1 \_I H I T S+ \\
787.864 * \text { } * K 1 \_D M I S S+ \\
391.801 \_K 1 \_D M I S S \_S T A L L S+ \\
28.794 * \_K 1 \_E X E C \_B U N D L E S
\end{array}
$$

\subsubsection{Validation}

To carry out the validation of the energy consumption model of the MPPA, a new application has been employed. To select the application, several considerations have been taken into account. First, the number of active resources needs to modifiable; hence, the resource-active consumption can vary. Secondly, the communications performed in the application have to be different from the $\mu$ benchmarks used to model this part. Finally, the processing kernel needs to be different from the ones used during the modeling of the contribution of the computation.

Considering these requirements, the application that has been chosen is GeMM application contained in Basic Linear Algebra Subprograms (BLAS), which is based on QQuan Ho'18]. Although this application performs matrix multiplications, contrary to the naive version used during the development of the computation model, it employs a highly optimized kernel written in assembly code. Additionally, the communication protocol of this application implements situations that have not been included in Section 5.5, e.g., multiple CCs accessing the IO in parallel. Specifically, the main characteristics of the application are:

- It uses single precision floating point data type (f loat).

- It multiplies A matrix transposed by B matrix $\rightarrow C=A^{T} * B$.

- Matrices A, B and C have 4,096×4,096 elements.

- The matrices are multiplied using blocks of $128 \times 128$ elements.

- To compute each C block, one C block is read and written, while 32 blocks of A and $B$ are only read from DDR, so, in total:

- 1,024 blocks of $C(128 \times 128$ elements $)$ are read and written.

- 32,768 blocks of A and B (128×128 elements) are read.

- Each C block is computed in a different cluster.

- Internally to the CC, first, the pixel block is divided among the active PEs, distributing the rows homogeneously. After that, each PE processes its part of the block using a highly optimized kernel, written in assembly code, that processes each block using sub-blocks of 4 rows by 8 columns. 
However, in the original implementation, not every combination of $\mathrm{CC} \beta$ and $\mathrm{PE} s$ was supported. As a result, to fully validate the energy consumption model, the application has been slightly modified to be able to execute it with every combination of active $\mathrm{CC} s$ and $\mathrm{PE}$. The modification consists in supporting non-uniform workload distributions inside the $\mathrm{CC}$ by processing the trailing rows using a naive matrix multiplication. These trailing rows appear when the 128 rows of the $\mathrm{C}$ block cannot be homogeneously distributed among the active PEs. For example, having 3 active PEs, two of them will process 42 rows, while the third one will process 44 rows. Therefore, as the kernel written in assembly processes 4 rows in each execution, the two first PEs will have 2 trailing rows each that are processed using the naive approach.

Considering the modified version of the GeMM application, during the validation process, each contribution composing the energy consumption model will present the following variability:

- Resource-active: the number of clusters involved in the execution of the GeMM application can take values from 1 to 16.

- Communication: the application will perform $\mathrm{IO}$ to $\mathrm{CC}$ transmissions when distributing the matrices among the $\mathrm{CC} s$, and $\mathrm{CC}$ to $\mathrm{IO}$ communications when they send back the result. Additionally, simultaneous reads and writes will be carried out when more than one CC is active.

- Computation: inside each cluster, the workload can present (or not) nonuniform workload distributions among PEs. Furthermore, each $\mathrm{PE}$ can execute the kernel written in assembly code or the naive matrix multiplication. Both variabilities depend on the active $\mathrm{PE}$.

The different situations referred to the computation are summarized in Table 5-14. In this table, each row corresponds to a number of active $\mathrm{PE}$ s but the columns are divided into two levels. On the one hand, to represent non-uniform distributions of the 128 rows to be processed inside each CC, there is a distinction between the first $N-1$ active $P E s$ and the Last $P E$. On the other hand, in order to consider the execution of different kernels, OptK columns contain the blocks of 4 rows processed by each $\mathrm{PE}$ using the kernel written in assembly code, while the Naive columns contain the trailing rows processed using the naive matrix multiplication.

To perform a thorough validation of the energy consumption model, a total amount of 256 testing points compose the validation experiment. In this regard, two levels of variability are considered: activating from 1 to $16 \mathrm{CC} s$ and, for each number of CCs, varying the number of active $\mathrm{PE}$ s from 1 to 16.

The results associated to these experiments are summarized in two figures. On the one hand, Figure 5-31 includes the time, power and energy consumption of the GeMM application in a unique plot gathering the 256 testing points. On the other hand, Figure 5-32 displays a comparison between the real measurement (in blue) and the energy consumption estimation (in red), again, for the 256 testing points. 
Table 5-14: Workload distribution in GeMM application with the number of active PEs per CC. Second column gathers the processing performed by the $N-1$ active PEs (optimized kernel blocks (OptK) or naive matrix multiplication rows (Naive)). Third column contains the processing performed by the last active $\mathrm{PE}$ (OptK blocks or Naive rows)

\begin{tabular}{|c|c|c|c|c|}
\hline \multirow{2}{*}{ \# of active PEs } & \multicolumn{2}{|c|}{$\mathrm{N}-1$ active $\mathrm{PE}$} & \multicolumn{2}{|c|}{ Last $P$} \\
\hline & OptK & Naive & OptK & Naive \\
\hline 1 & 0 & 0 & 32 & 0 \\
\hline 2 & 16 & 0 & 16 & 0 \\
\hline 3 & 10 & 2 & 11 & 0 \\
\hline 4 & 8 & 0 & 8 & 0 \\
\hline 5 & 6 & 1 & 7 & 0 \\
\hline 6 & 5 & 1 & 4 & 3 \\
\hline 7 & 4 & 2 & 5 & 0 \\
\hline 8 & 4 & 0 & 4 & 0 \\
\hline 9 & 3 & 2 & 4 & 0 \\
\hline 10 & 3 & 0 & 5 & 0 \\
\hline 11 & 2 & 2 & 4 & 2 \\
\hline 12 & 2 & 3 & 4 & 2 \\
\hline 13 & 2 & 1 & 5 & 0 \\
\hline 14 & 2 & 1 & 2 & 3 \\
\hline 15 & 2 & 0 & 4 & 0 \\
\hline 16 & 2 & 0 & 2 & 0 \\
\hline
\end{tabular}

As can be seen, in Figure 5-31, the total amount of active $\mathrm{PE}$ per $\mathrm{CC}$ has a huge impact in both the time and the power consumption and, consequently, the energy follows this trend. This variation is associated to the non-uniform workload distribution and, additionally, to the use of a naive matrix multiplication to process the trailing rows. As a result, analyzing Figure 5-32, it can be observed that this variability is perfectly represented by the model estimation.

Going deeper in the analysis, Figure 5-33 displays the information for the experiments in which $16 \overline{\mathrm{CC}} s$ are used. The objective of this study is to validate the model when there is not a predominant part of the model that overcomes the other two. As can be seen, although the estimation using only the resource-active contribution (red line) already match the trend of the estimation (blue line), only the estimation using the complete model (purple line with crosses as markers) is able to provide an accurate representation of the energy consumed by the platform. Specifically, the total estimation is almost identical to the real measurement.

Finally, in order to quantitatively analyze the estimation, Table 5-15 contains the average relative error, the coefficient of determination $R^{2}$ and the $\mathrm{SNR}(\mathrm{dB})$ obtained for the whole set of experiments used during the validation procedure. As can be seen, the average relative error is below $5 \%$ and the data trend representation $\left(R^{2}\right)$ is above $99 \%$. Hence, the applicability of the energy consumption model when using an application that has not been used for its extraction has been demonstrated. 


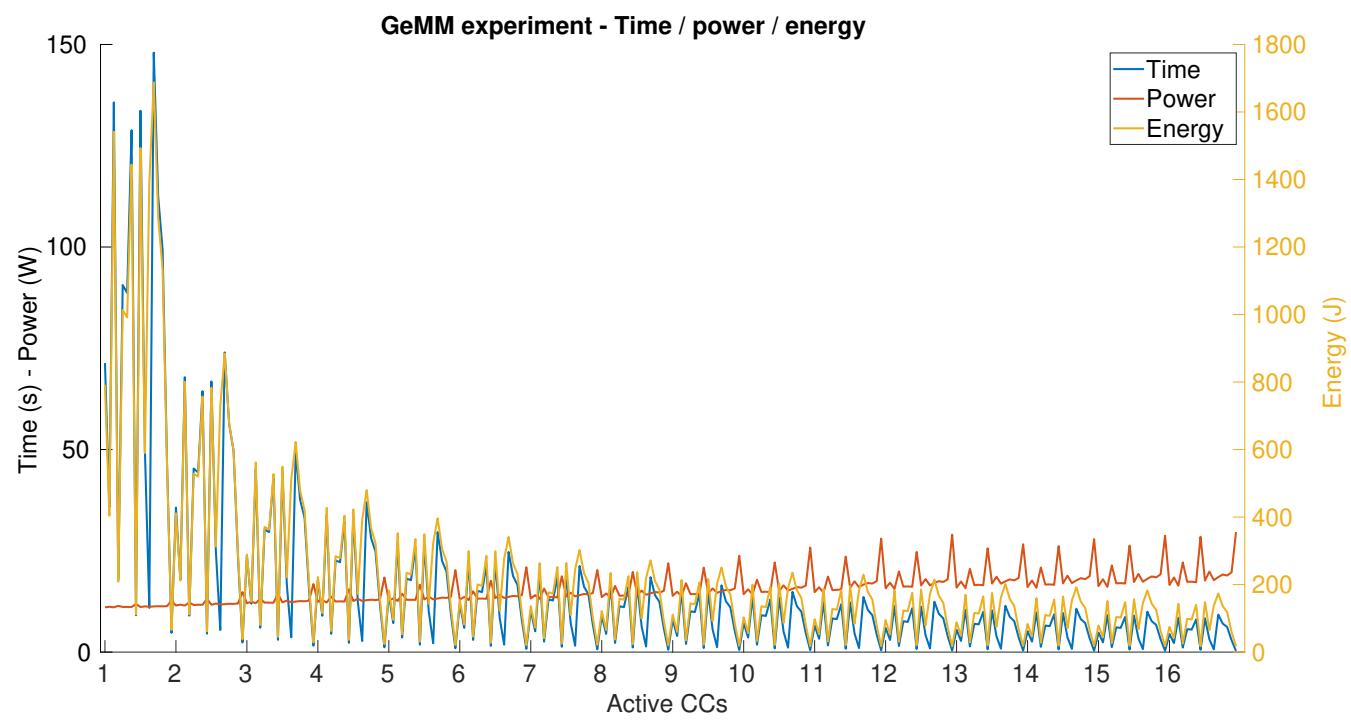

Figure 5-31: Model validation-GeMM experiment analysis

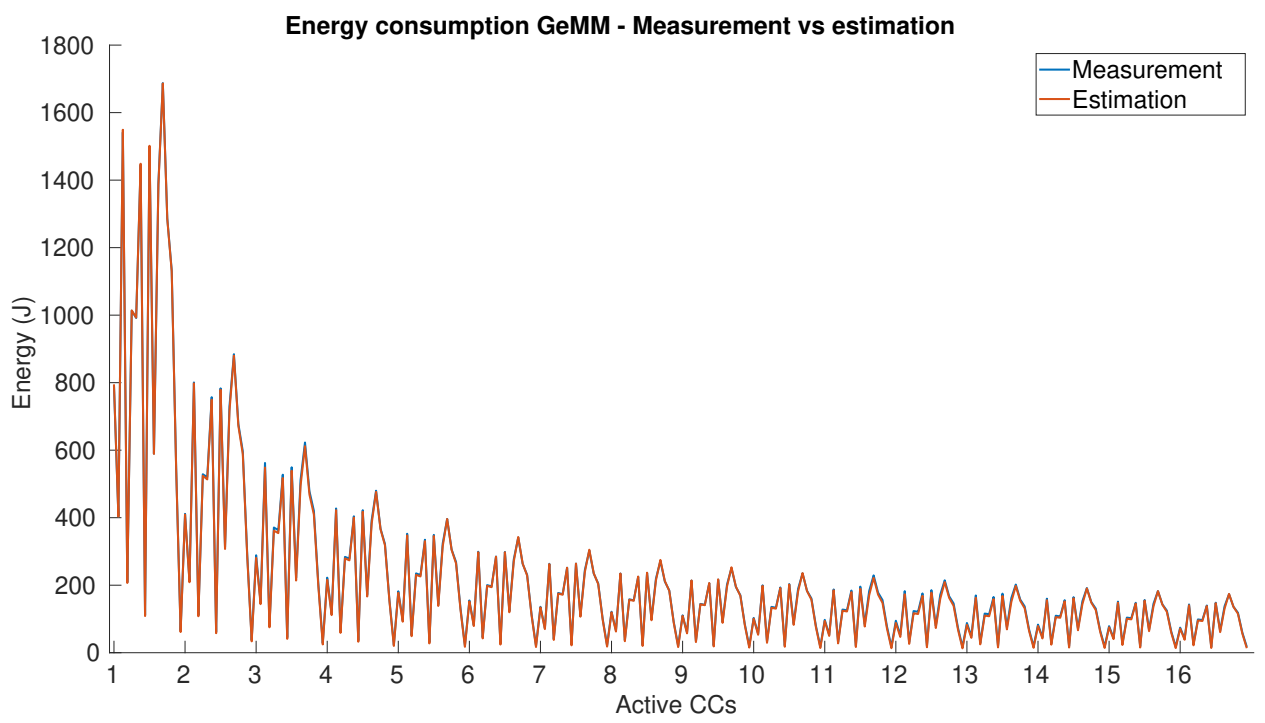

Figure 5-32: Model validation-GeMM experiment energy measurement vs estimation

Table 5-15: Platform model validation with $\mathrm{GeMM}$ considering average relative error (in \%), the coefficient of determination $R^{2}$ and $\operatorname{SNR}(\mathrm{dB})$

\begin{tabular}{|c|c|c|c|}
\cline { 2 - 4 } \multicolumn{1}{c|}{} & Average relative error (\%) & $R^{2}$ & SNR(dB) \\
\hline MPPA model & 4.765 & 0.999 & 37.18 \\
\hline
\end{tabular}




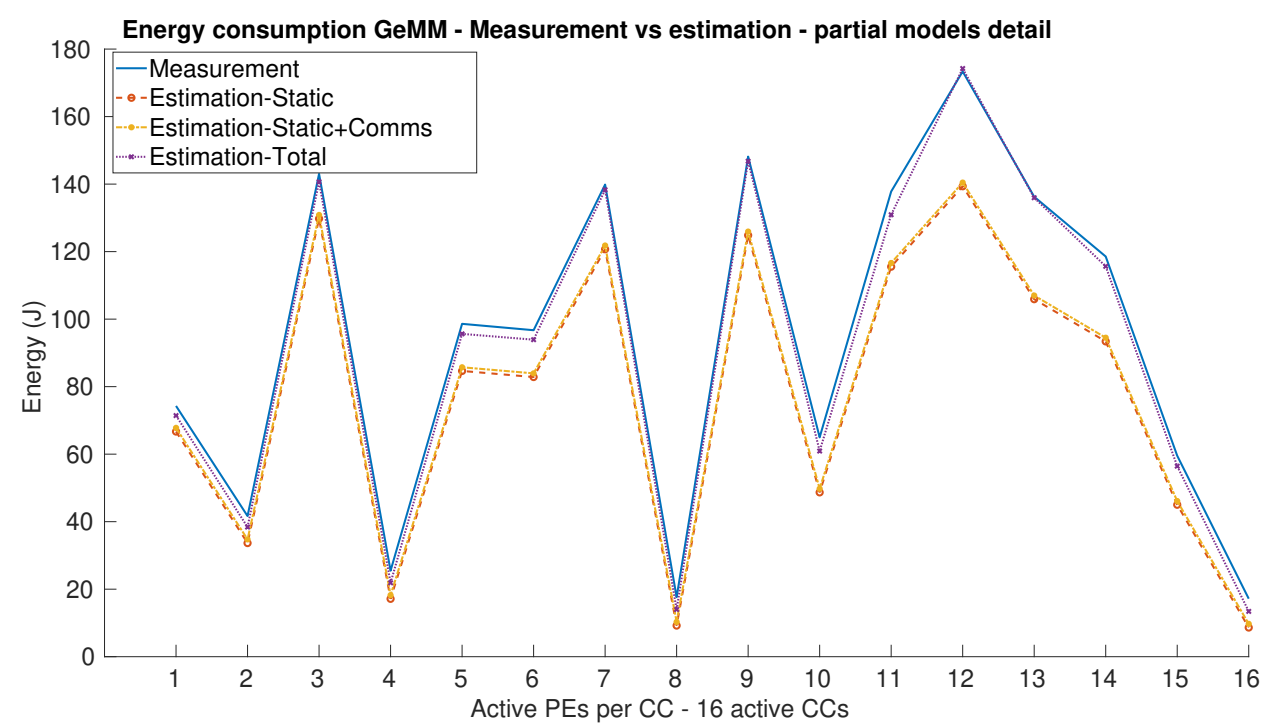

Figure 5-33: Model validation - GeMM experiment energy measurement vs estimation detail using $1 6 \longdiv { \mathrm { CC } }$

\subsection{Discussion}

In this chapter, a new modeling methodology aiming at building application-agnostic energy consumption models using a high-level approach has been presented as one of the original contributions of this thesis. This modeling approach has been employed to extract an energy consumption model of the MPPA-256-N many-core platform. Specifically, this model is the combination of 3 different energy consumption contributions modeled using a set of $\mu$ benchmarks. Furthermore, during the validation using a GeMM application, which was not part of the set of $\mu$ benchmarks, an average relative error below $5 \%$ has been obtained.

In order to build the model, a new PAPI component has been developed to transparently access MPPA PMC information. This new component is considered an original instrumental contribution of this $\mathrm{PhD}$.

The model built in this chapter will be used in the real use-case of this $\mathrm{PhD}$ during Chapter 7. On the one hand, at design time, the model will be used to estimate the energy consumption of the HSI application after profiling its execution, as explained in Subsection 7.3.4. On the other hand, in Subsection 7.4.3 and Subsection 7.4.4. its applicability in a runtime context to perform on-the-fly energy consumption estimations is demonstrated. By doing so, a new validation of the model accuracy is performed through a real use-case. 


\section{ENERGY-AWARENESS IN STREAMING APPLICATIONS}

After introducing PAPIFY monitoring infrastructure in Chapter 4 and the energy modeling methodology in Chapter 5, this chapter presents a procedure to optimize the system energy consumption (Figure 6-1). To do so, both PAPIFY and the energy model are combined with an energy-aware decision making protocol to build a CERBERO-like multi-objective optimization loop focused on optimizing energy consumption.

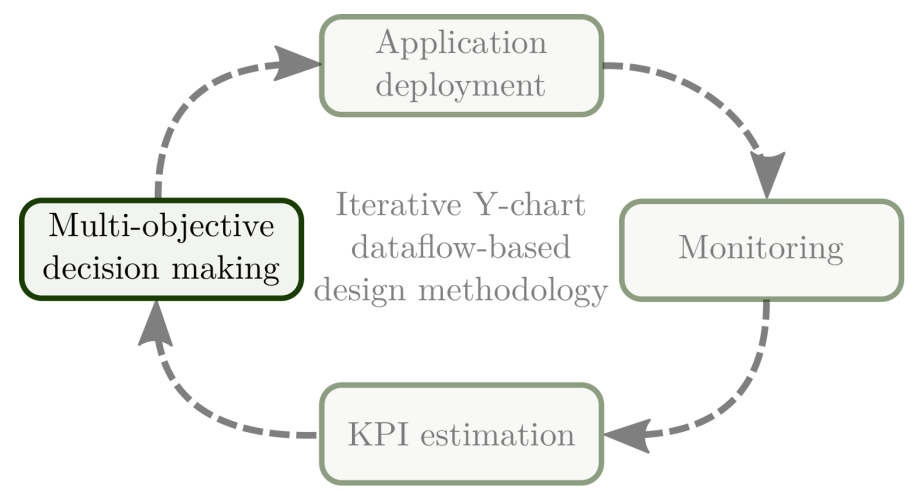

Figure 6-1: Iterative Y-chart dataflow-based design methodology - Decision making

To build this multi-objective optimization loop, first, Section 6.1 introduces the iterative approach proposed in this $\mathrm{PhD}$, which is composed of 4 blocks: monitoring, KPI estimation, energy-aware decision making and application deployment. Afterwards, Section 6.2 deeply analyzes the block associated to energy-aware decision making, focusing on the idea of tuning latency-based mapping/scheduling algorithms to obtain an energy-efficient system.

Since this approach can be applied either at design time or at runtime, two different loops are presented. The design time energy-aware optimization loop will be presented in Section 6.3. This part will address the energy consumption optimization of dataflow applications when exploring the design space (DSE step). Secondly, the energy-aware optimization loop at runtime is introduced in Section 6.4. In this case, the system is provided with a self-aware runtime resource manager, which will be able to characterize the application execution and take energy-aware decisions on-the-fly.

Finally, Section 6.5 sums up the main contributions explained in this chapter. 


\subsection{Energy-awareness overview}

The energy-aware optimization loop proposed in this $\mathrm{PhD}$ follows Figure 6-2, which is a simplification of the CERBERO one, presented in Subsection 2.3.4. This simplification targets the optimization of streaming software applications, while the CERBERO one focuses on hardware implementations [Palumbo'19a]. Specifically, these streaming applications are characterized by their iterative nature, e.g., video processing. That is, they can be considered applications running infinitely where a minimum performance objective (number of iterations of the application executed per second) must be achieved. For example, in a video application, a minimum number of Frames Per Second (FPS) could be required as its performance objective.

To be able to easily adapt this approach to different development frameworks that employ latency-based mapping and scheduling algorithms, the energy-aware optimization loop will consider the mapping/scheduling task a black box and will work on top of it. In this line, as detailed in Section 6.2, this technique, called energyaware tuning, will iteratively modify the system configuration by means of changing the number of active PEs.

In a nutshell, the energy-aware optimization loop, as shown in Figure 6-2, is a particularization of the loop shown in Figure 3-4 including energy-awareness as part of the multi-objective decision making protocol. As a result, it is composed of 4 steps:

- Monitoring: PAPIFY, the application monitoring infrastructure presented in Chapter 4, is used to profile the application for both execution time and PAPI events. This profiling is performed independently for each actor when being executed on each type of $\mathrm{PE}$.

- KPI estimation: each actor composing the dataflow graph is characterized in terms of timing and energy consumption. To compute the latter, PAPI events are correlated to the energy consumed on each type of PE by means of the dynamic energy consumption model developed in Section 5.6.

- Energy-aware decision making: in this block, first, the achieved performance is computed together with the total energy consumed by the system. Secondly, the achieved performance is compared to the performance objective and the necessity of changing the system configuration is evaluated.

- Application deployment: if the configuration is not valid/optimum, the application workload is redistributed among the platform resources considering a different system configuration, i.e., a different number of active PEs. 


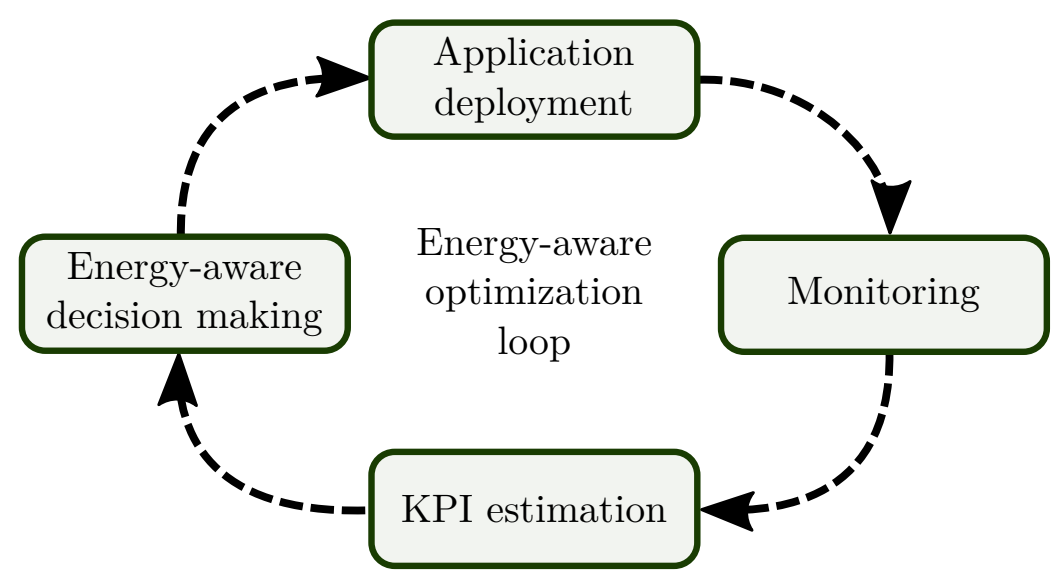

Figure 6-2: Energy-aware optimization loop

\subsection{Energy-aware tuning}

In the context of dataflow Y-chart design methodologies, two inputs are employed to automatically generate the system: an application dataflow graph that can be analyzed to extract its intrinsic parallelism, and a description of the target architecture to infer the available parallelism opportunities. This information is especially useful when, during the system generation, the main objective is to minimize the system latency.

For example, in Figure 6-3, an example of application deployment that can be performed following a latency-based approach is shown. On the application side top-left side of the figure-, an application graph with 4 actors is displayed. In this application, for each iteration of the dataflow graph, Actor $A$ and Actor $B$ can be executed in parallel and generate 4 tokens each; Actor $C$ consumes one token per input and generates one token through its output every time it is fired, meaning that 4 instances of this actor need to be executed for the graph to be consistent; finally, Actor $D$ consumes 4 tokens, so it needs to wait until the 4 instances of Actor $C$ are executed to be launched. On the architecture side -top-right side of the figure-, the platform is defined as a 4-Core architecture interconnected through a shared memory, meaning that a maximum parallelism of 4 is supported. Finally, when the system is generated using a latency-based mapping/scheduling algorithm, one possible output is the one shown in the bottom part of Figure 6-3. In this case, as can be seen, $P E 2$ and $P E 3$ execute only one instance of Actor $\mathrm{C}$, being idle most of the time.

These approaches do not consider a performance objective or the energy consumption, and tend to provide the maximum performance, i.e., the maximum graph executions per second. Regarding the performance, for example, in video applications, reaching frame rates (performances) above 60 FPS is not useful for standard videos, as the human eye is not able to process it. Specifically, frame rates above 60 FPS are used to record slow-motion videos. From the energy consumption point of view, if no other technique such as power or clock gating is 


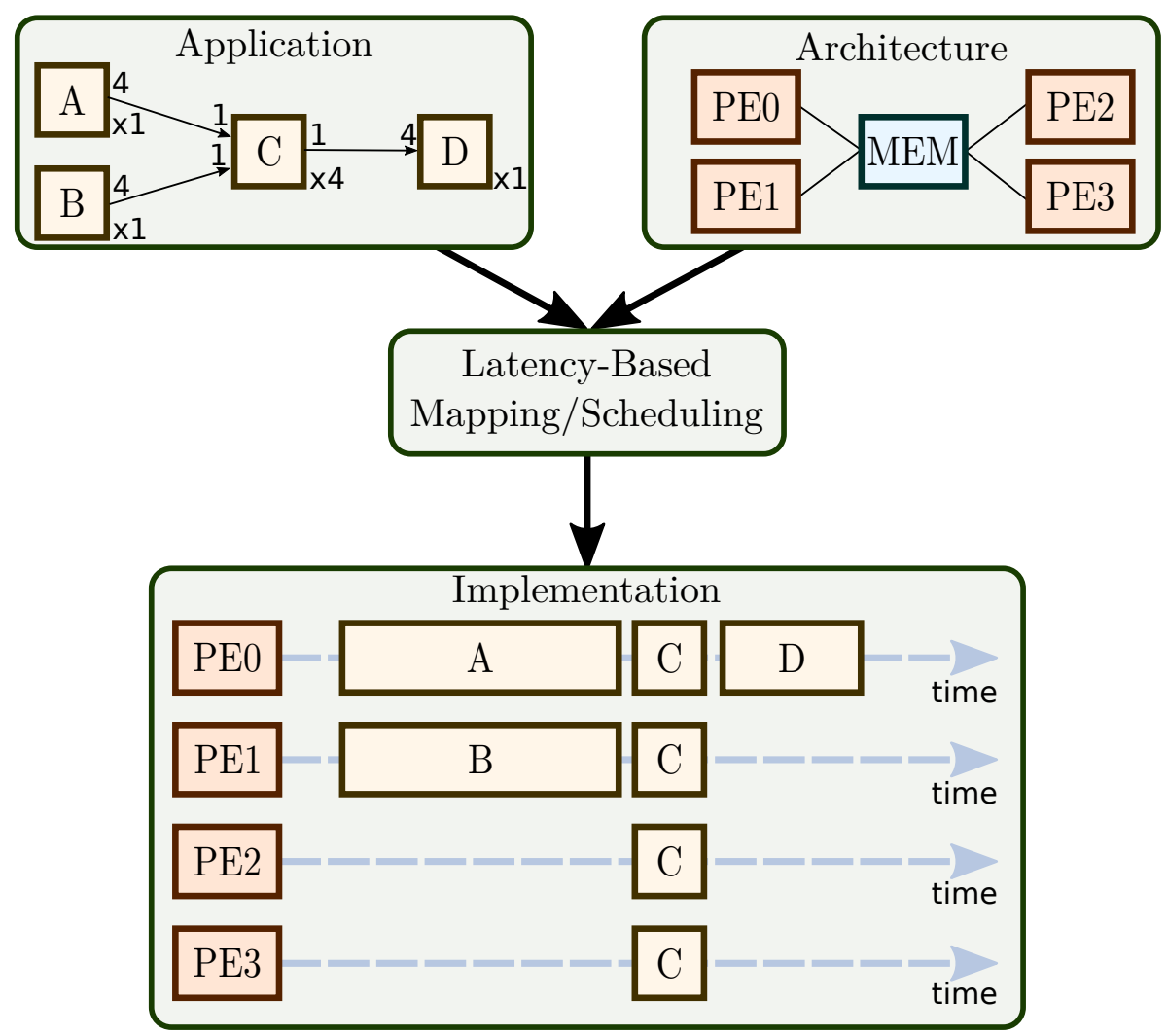

Figure 6-3: $\mathrm{SDF}$ Y-Chart flow example

applied, performances above the objective lead to a waste of energy, as the dynamic consumption, which is linked to the workload, will be higher. To deal with these aspects, in the literature, DVFS techniques are usually applied to adapt the reached performance to the objective one [Nogues'15].

Another approach is to implement mapping/scheduling algorithms performing multi-objective DSE where a Pareto front is provided. A Pareto front is composed of a set of solutions where latency and energy consumption are balanced and, depending on the user requirements, the most suitable solution is selected [Piscitelli'12. However, contrary to latency-based mapping/scheduling algorithms, the multiobjective approach is not fully extended within the Y-chart design frameworks.

In this line, during this $\mathrm{PhD}$, a modification of the latency-based mapping/scheduling algorithm is proposed. Specifically, an energy-aware loop is built surrounding the latency-based mapping/scheduling block, which is considered a black box. This mechanism will provide an energy-efficient implementation where a user-defined performance objective is reached. This approach, called energy-aware tuning, is shown in Figure 6-4, where the previously existing block is represented in blue and the extension in orange. This loop iterates searching a system configuration, i.e., a number of active $\mathrm{PE}$, that optimizes the energy consumption while reaching the performance objective. To do so, the latency-based mapping/scheduling algorithm, which is kept unmodified, deploys the application onto the available resources and, for 
each tested system configuration, its output is evaluated in terms of performance and energy consumption. If the performance obtained is not enough, the configuration is automatically discarded and the searching continues. On the contrary, when the performance is reached, the configuration with the lowest energy consumption is stored, while the others are also discarded. By doing so, different implementations are tested and, once the searching finishes, the optimum configuration can be selected.

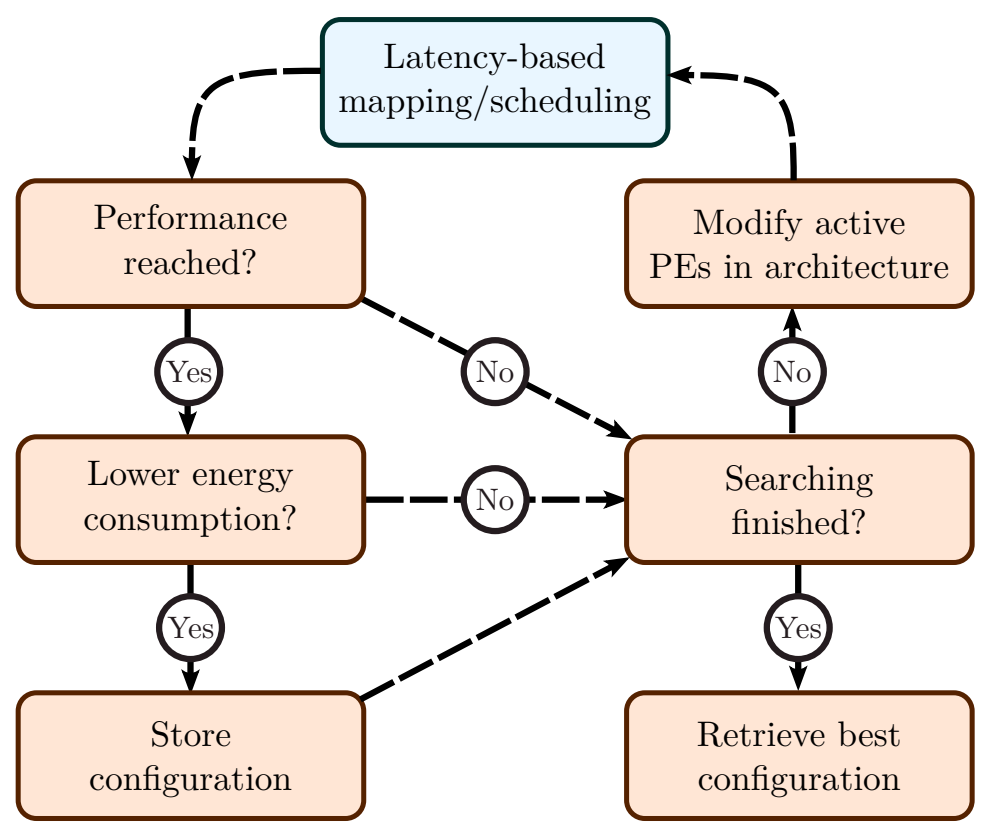

Figure 6-4: Energy-aware tuning algorithm block diagram

To illustrate this idea, the energy-aware tuning algorithm can be used in the example explained in Figure 6-3. If, in this case, the performance reached by the latency-based mapping/scheduling algorithm surpasses the performance objective, a new implementation where the energy consumption is reduced could be obtained thanks to the new methodology. In Figure 6-5, both implementations are compared, Figure 6-5a representing the implementation where only latency has been considered and Figure 6-5b showing one possible solution including energy-awareness. In this case, two $\mathrm{PE}$ are disabled, which may lead to energy savings.

To take advantage of developers' knowledge about the system, the searching protocol of the tuning algorithm can be configured using two parameters:

- Starting point: this parameter sets the first configuration that is tested in the searching algorithm and it can take the following values:

- First: starts the searching using the least possible active PEs.

- Middle: starts with half of the PEs active and half of them disabled.

- Max: starts the searching with all the PEs enabled.

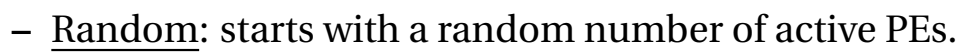




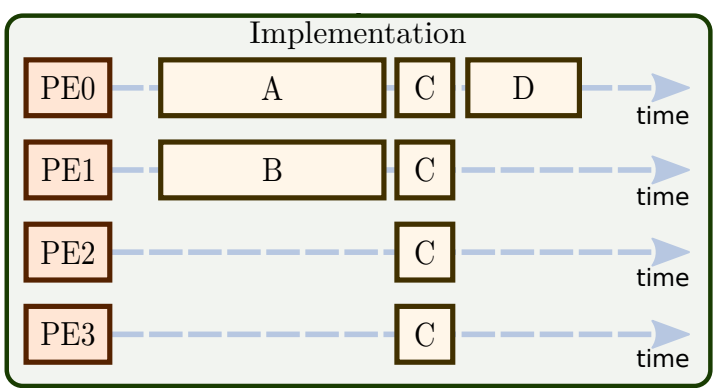

(a) Latency-based

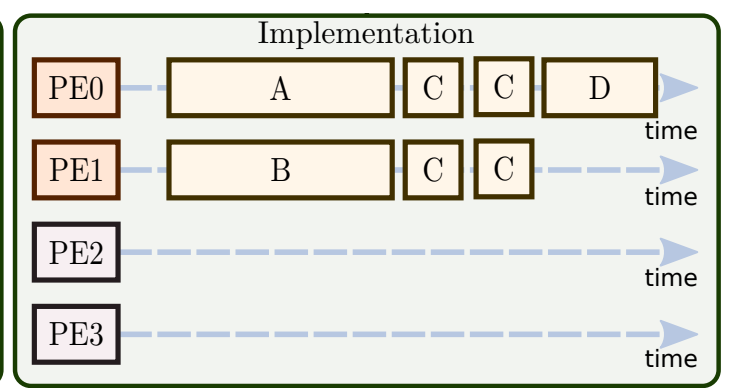

(b) Energy-awareness

Figure 6-5: Implementation comparative: (a) latency-based vs (b) energy-awareness

- Searching mode: this parameter defines the type of searching that is performed:

- Thorough: performs a full/DSE, increasing/decreasing the number of active PEs one by one if the current configuration is below/above the performance objective, respectively.

- Binary: searches the optimum configuration using a heuristic binary search. This searching methodology consists in dividing in half the available configurations that can contain the optimum configuration. In this case, the available configurations has to be sorted considering the number of active PEs before starting the process.

To exemplify one searching process of the energy-aware tuning algorithm, a graphical example is provided in Figure 6-6. This example considers a total amount of 8 PEs, a performance objective of 15 , and the searching has been configured as follows: 1) the starting point has been set to Max and 2) the searching mode has been set to Binary. In this case, 4 different configurations -represented with numbers 1, 2, 3 and 4 in the Tested configuration order row- have been tried using 8, 4, 2 and 3 PEs. For each of them, the Performance achieved is shown in a gray circle above each tested configuration. As a solution, the configuration using $3 \mathrm{PE}$ is selected, since it is the one using the least $\mathrm{PE} s$ among the ones above the performance objective.

Finally, as introduced in Section 6.1, in this PhD, the energy-aware tuning algorithm has been combined with both a monitoring infrastructure and an energy estimation block so as to build an energy-aware optimization loop. This loop can be included in different frameworks and applied at either design time or runtime. Specifically, in Section 6.3, its integration with a design time framework, PREESM, is explained, while Section 6.4 details its adaptation to a runtime resource manager,SPiDER. 


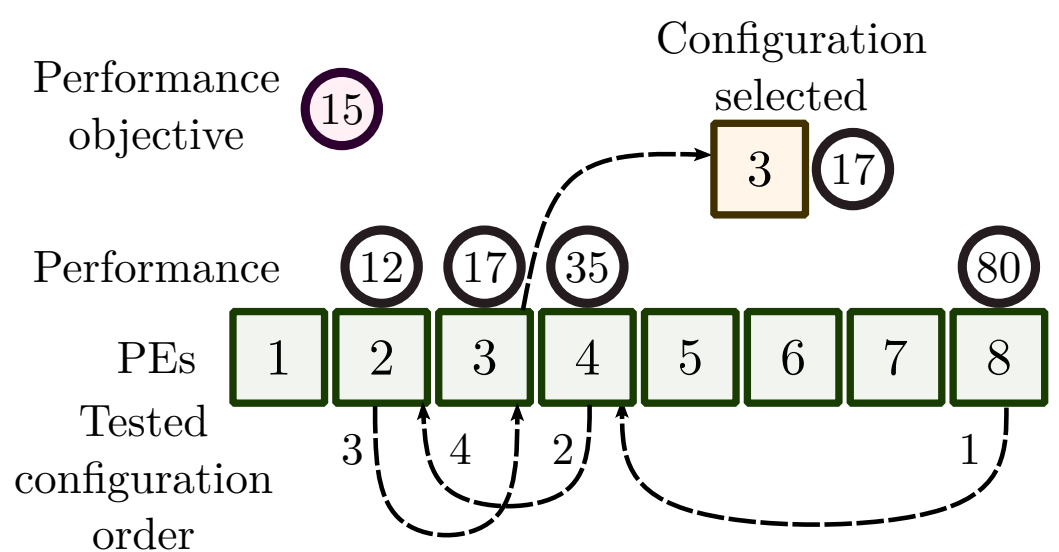

Figure 6-6: Binary search example. Starting point Max and performance objective 15

\subsection{Design time optimization loop}

In this section, the implementation of the energy-aware optimization loop in PREESM design framework is explained. To guide the explanation, first, an overview of how the optimization loop introduced in Section 6.1 has been included within the real workflow of PREESM is presented. Then, the details associated to the implementation of each block within the loop are provided.

In this line, the optimization loop proposed for the design time is summarized in Figure 6-7. In this figure, standard PREESM blocks are represented in blue, the extensions in orange and the blocks that already existed within preesm, but which have been modified combine both colors. In the standard workflow of PREESM, 3 sources of information are used to generate the systems: the application graph, the architecture definition and a set of user-defined constraints. These constraints are considered during the DSE to generate the application deployment. This workflow has been enhanced with monitoring capabilities, where the real execution of the application is profiled, and a KPI estimation block to update the information of the user-defined constraints. By doing so, the optimization loop is closed and an iterative system development procedure is defined, where PREESM can work with real profiling information to enhance its decisions. As a result, the steps composing the energyaware optimization loop are linked to Figure 6-7 as follows:

- Monitoring: in this case, the systems generated with PREESM are monitored using PAPIFY. In this monitoring process, both execution time and PAPI events are gathered for each actor when being executed on each type of $\mathrm{PE}$.

- KPI estimation: this block has been embedded within the scenario of PREESM, where the user-defined constraints are specified. Specifically, timing information is updated in Timings tab, while the energy consumption is included in a new dedicated tab called Energy. 


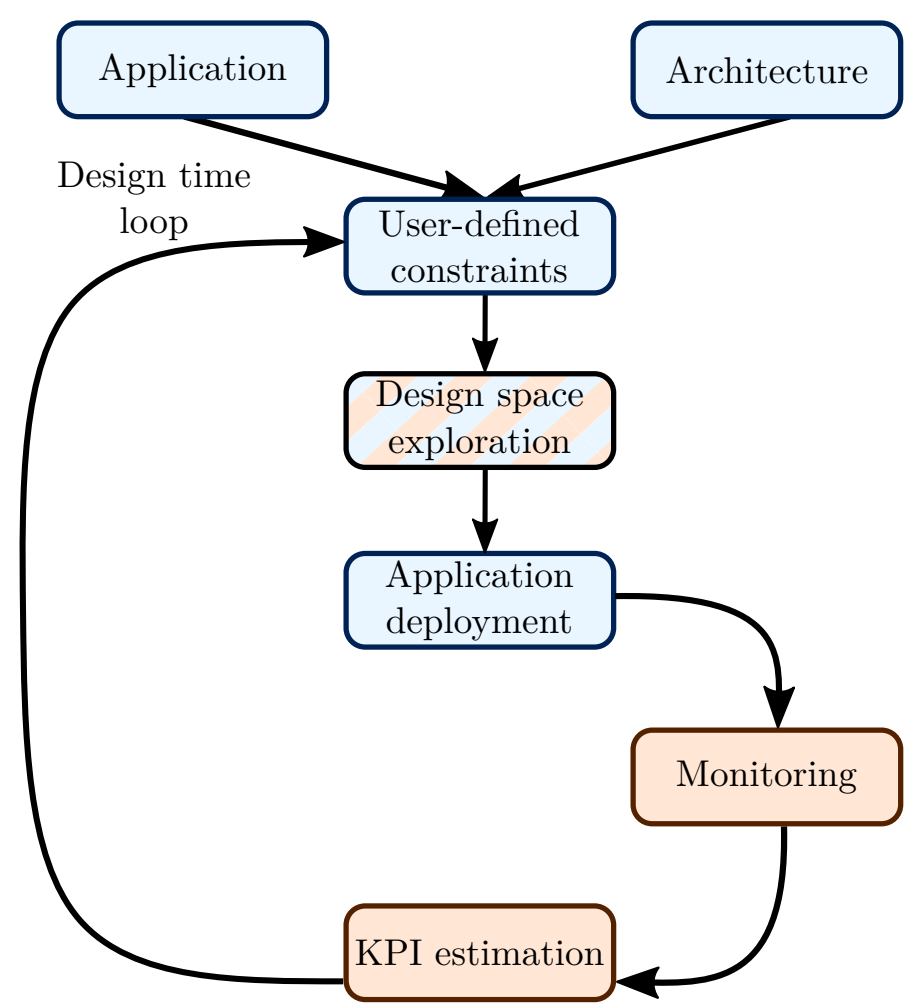

Figure 6-7: Y-Chart dataflow design flow at design time

- Energy-aware decision making: this part has been embedded within the DSE block. The energy-aware tuning algorithm will explore the design space considering the information that is available on the scenario file of PREESM. For this reason, the information about timing and energy consumption will be updated and refined using data extracted during real executions.

- Application deployment: the output of the DSE block is the application deployment considered in each iteration.

Once the energy-aware optimization loop in PREESM has been introduced, the implementation details of each block composing the loop are provided.

First, in order to perform an application profiling, the PAPIFY monitoring infrastructure explained in Chapter 4 is employed. During this procedure, real application executions are characterized in terms of 1) execution time and 2) generated PAPI events. The former is used to provide PREESM with accurate measurements of the time required to execute each actor within the application graph. The latter, in turn, should be matched with the energy consumption model developed using the methodology proposed in Section 5.6. To do so, the necessary PAPI events generated by each actor to estimate the energy consumption associated to the computation are gathered. This profiling information is stored in a dedicated folder called papifyoutput during the application execution so as to update the scenario when needed. 
Regarding KPI estimation, two figures of merit need to be updated: timing and energy consumption. To update timing information, PREESM provides a tab devoted to this aspect in the scenario file, which is shown in Figure 6-8. In this tab, the time required to execute each actor is shown in the Value column (black dashed-dotted rectangle). This value is set for each actor (blue dotted rectangle) and for each type of $\mathrm{PE}$ (green dashed rectangle). In this $\mathrm{PhD}$, the Timings tab has been extended so as to include a parser to automatically update the timing associated to each actor-PEpair directly from papify-output. This parser is launched when using the Import papifyoutput button (red rectangle). It should be noted that, when using this parser, the timing unit of the actor execution is assumed to be the microsecond.

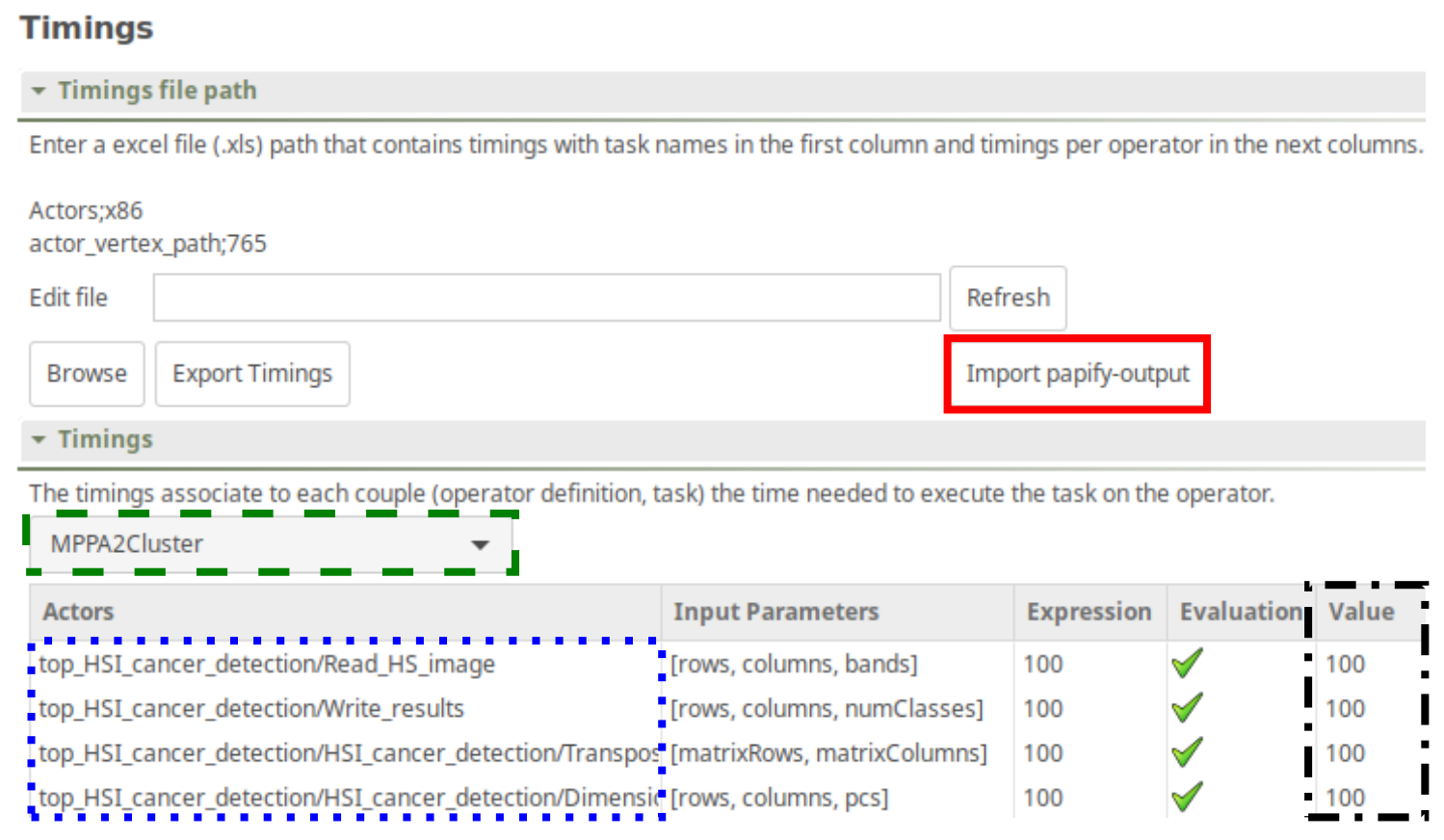

Figure 6-8: Timings tab in PREESM scenario

In the case of the energy consumption information, a new tab called Energy has been added within PREESM scenario, as shown in Figure 6-9. As can be seen, it has been divided into 5 different sections:

- Energy file path: used to upload the energy consumed by each actor when executed by each type of $\mathrm{PE}$. To do so, the values can be included by hand or using an automatic parser, which will be explained later, to directly estimate the energy from the papify-output folder.

- Performance objective: since the energy-aware tuning requires a performance objective, this value is included in this section in graph executions per second.

- Platform power consumption: the resource-active power consumption of the target platform is included in this section, following the model presented in 


\section{CHAPTER 6. ENERGY-AWARENESS IN STREAMING APPLICATIONS}

\section{Energy}

Energy file path

Enter an excel file (.xIs) or a .papify path that contains energy with task names as rows and operator definitions as columns. The Energy actors $<>$ PE type section will be updated accordingly. Additionally, if a papify-output folder is selected, the model from PAPIFY tab will be applied to parse energy values

\begin{tabular}{|l|l|l|}
\hline Edit file & Refresh \\
\hline Browse Export & Import papify-output \\
\hline
\end{tabular}

\section{Performance objective}

This section contains the performance objective to reach during the energy-aware mapping/scheduling

\begin{tabular}{|l|l|}
\hline Parameter & Value \\
\hline Objective - in executions per second: & 50.0
\end{tabular}

\section{Platform power consumption}

This section includes all the information related to the static power consumed by the platform. Specifically, "Base" represents the baseline of power and, the rest represents the power consumption increment when activating one new PE of a specific type

\begin{tabular}{|l|l|}
\hline Operator Definition & Associated power [W] \\
\hline Base & 10.776 \\
\hline MPPA2Cluster & 0.257 \\
\hline MPPA2IO & 0.0 \\
\hline
\end{tabular}

\section{Communication energy consumption}

This section includes all the information related to the energy consumption due to data communication, Specifically, the energy required to transmit 1 byte from 1 type of PE to another type of PE [PJ]

\begin{tabular}{|l|l|l|}
\hline Source $\backslash$ Destination & MPPA2Cluster & MPPA2IO \\
\hline MPPA2Cluster & 118021.42 & 121743.8 \\
\hline MPPA2IO & 126688.71 & 130411.09 \\
\hline - Energy actors $\leftrightarrow \rightarrow$ PE type & & \\
\hline
\end{tabular}

This section includes all the information related to energy consumption of each actor when being executed on each type of processing element. MPPA2Cluster

\begin{tabular}{|l|l|}
\hline Actors & Value \\
\hline top_HSI_cancer_detection/Read_HS_image & 1.0 \\
\hline top_HSI_cancer_detection/Write_results & 1.0 \\
\hline top_HSI_cancer_detection/HSI_cancer_detection/TransposeMatrix & 1.0 \\
top_HSI_cancer_detection/HSI_cancer_detection/DimensionalityReduction/WritePcaResults & 1.0 \\
top_HSI_cancer_detection/HSI_cancer_detection/SpatialSpectralFiltering/DecisionMaking & 1.0
\end{tabular}

Figure 6-9: Energy tab in PREESM scenario 
Section 5.4. Specifically, Base power is linked to the constant value, which is independent of the active resources, while, in this case, MPPA2Cluster and $M P P A 2 I O$ rows represent the power associated per active $\mathrm{PE}$.

- Communication energy consumption: the energy required to transmit data among platform resources has to be included, following the model presented in Section 5.5. In this case, each row in the table is associated to the type of source, while each column is linked to the destination.

- Energy actor $\leftrightarrow$ PE type: the energy associated to the execution of each actor on each type of PE can be included in this part.

In order to facilitate the update of the energy consumed when executing each actor on each type of $\mathrm{PE}$, the process to estimate the energy consumed from PAPI events has been automated by means of a parser. This automatic parser is launched using the Import papify-output button shown in Figure 6-9 (Energy file path section). To be precise, the estimation is carried out using Equation 6-1, which is compliant with the energy consumption model associated to the computation contribution, as explained in Section 5.6. Figure 6-10 displays an example, using the MPPA model, of how the models are defined within PREESM framework. As this model is composed of PAPI events, the table used to define it can be found in PAPIFY tab. This table gathers all the available events in the columns and the different PE types in the rows.

$$
\text { Energy }_{P_{\text {type }}}[\mu \mathrm{J}]=a_{0} * P A P I_{\text {event }_{0}}+a_{1} * P A P I_{\text {event }_{1}}+\ldots+a_{N} * P A P I_{\text {event }_{N}}
$$

\begin{tabular}{|c|c|c|c|c|}
\hline \multicolumn{5}{|c|}{ In this section, the energy model for each type of PE is included. The events with a value different to 0 will be considered as valid and taken into account } \\
\hline PE Type \ PAPI event & k1_mppa2::-_K1_IHITS & k1_mppa2::_K1_DMISS & k1_mppa2::-_K1_DMISS_STALLS & k1_mppa2:::_K1_EXEC_BUNDLES \\
\hline MPPA2Cluster & $5.9311 \mathrm{E}-5$ & $7.2635 E-5$ & $3.1127 \mathrm{E}-5$ & $6.4572 E-5$ \\
\hline MPPA2IO & $4.22652 E-4$ & $7.87864 \mathrm{E}-4$ & $3.91801 \mathrm{E}-4$ & $2.8794 \mathrm{E}-5$ \\
\hline
\end{tabular}

Figure 6-10: Energy consumption computation model table in PREESM interface

Finally, the energy-aware decision making block has been embedded within the DSE task of PREESM. In this part, the energy-aware tuning algorithm -explained in Section 6.2- is used to consider energy consumption within the parameters to be optimized during the system generation. This task extracts the information from the scenario of PREESM. That is, it uses the timing measured with PAPIFY and the energy consumption estimated with all the parts of the model. To be precise, since PREESM simulates the application deployments proposed by the latency-based mapping/scheduling algorithm to estimate its performance, the energy-aware tuning algorithm predicts its associated energy consumption based on this simulation. 
As a result, the decision making block provides as an output the application deployment that consumes the least energy, while reaching the performance objective, considering the information that is currently available in the scenario.

\subsection{Runtime optimization loop}

This section details the runtime energy-aware optimization loop implemented in SPiDER runtime resource manager. In this case, the objective is to perform on-the-fly energy-aware optimizations based on real-time information. Similarly to the previous section, first, a general overview of how the optimization loop explained in Section 6.1 works in the real workflow of SPiDER is provided. After this, the different aspects of each block composing the loop are detailed.

In this regard, the runtime optimization loop in SPiDER is summarized in Figure 6-11, again, displaying in blue the already existing blocks and in orange the ones associated to energy-awareness extension. As can be seen, the generation of the system remains the same, but the application is generated only once. In the case of runtime optimization loop, the application and the architecture are combined under the set of user-defined constraints and an application deployment is generated only once after performing a DSE procedure. After this, the optimization loop itself has been included as the combination of monitoring of the current application deployment, KPI estimation considering this information and a runtime decision mechanism where energy is one of the parameters to be optimized.

As a result, the different blocks composing the energy-aware optimization loop are linked to the SPiDER workflow as follows:

- Monitoring: in this case, again, PAPIFY is used to retrieve performance information of both timing and $\mathrm{PMC}$ s from each actor when being executed on each PE, i.e., each LRT.

- KPI estimation: this part is linked with the homonymous block. Specifically, timing and energy consumption data are directly embedded within the characterization that SPiDER has of both the application and the architecture.

- Energy-aware decision making: SPiDER runtime decisions block has been modified so as to test a different system configuration in each iteration using the energy-aware tuning mechanism.

- Application deployment: the standard latency-based mapping/scheduling algorithm used by SPiDER generates the application deployment as usual. 


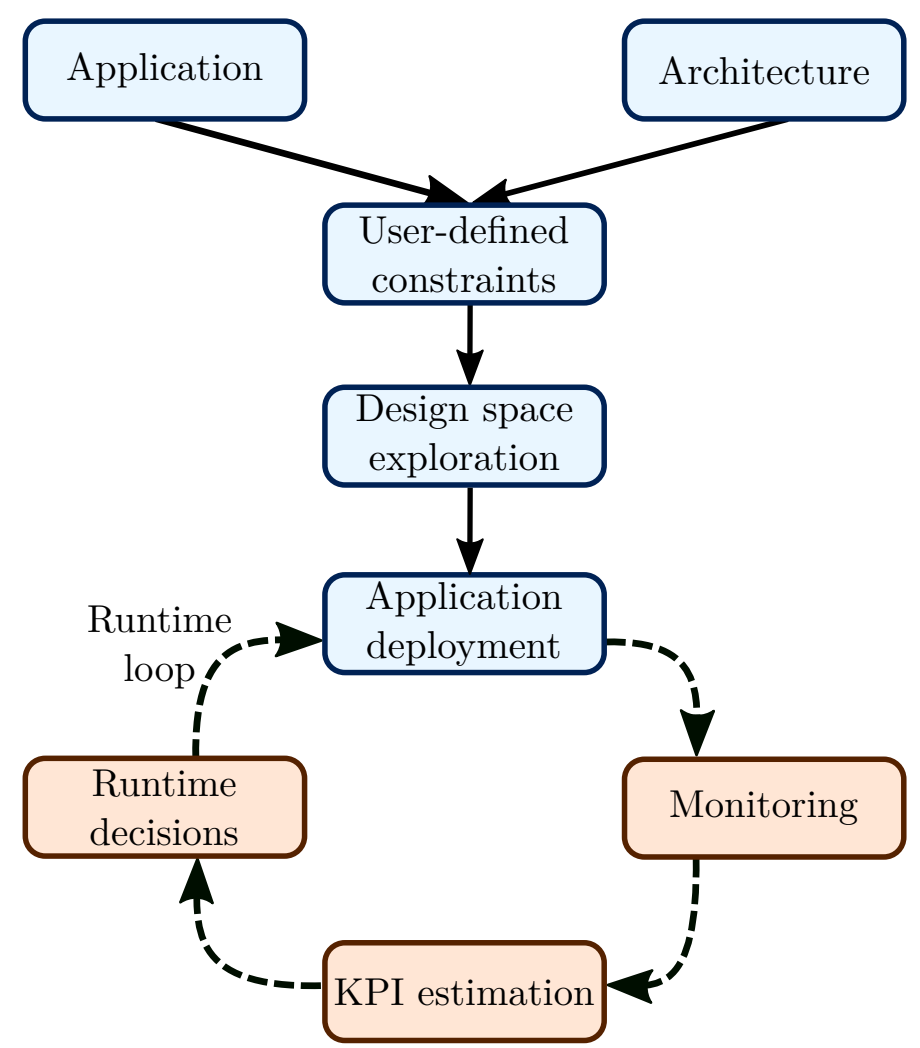

Figure 6-11: Y-Chart dataflow design flow at runtime

In particular, Figure 6-12 represents the details of the new SPiDER workflow, including the energy-aware runtime optimization loop. Specifically, to simplify its explanation, this figure represents a version of the loop where only one LRT is used. In this figure, the original SPiDER blocks are represented in blue, while the ones related to the inclusion of the energy-aware optimization loop are displayed in orange. During the rest of the section, this figure will be used to lead the explanation of the different blocks composing the loop.

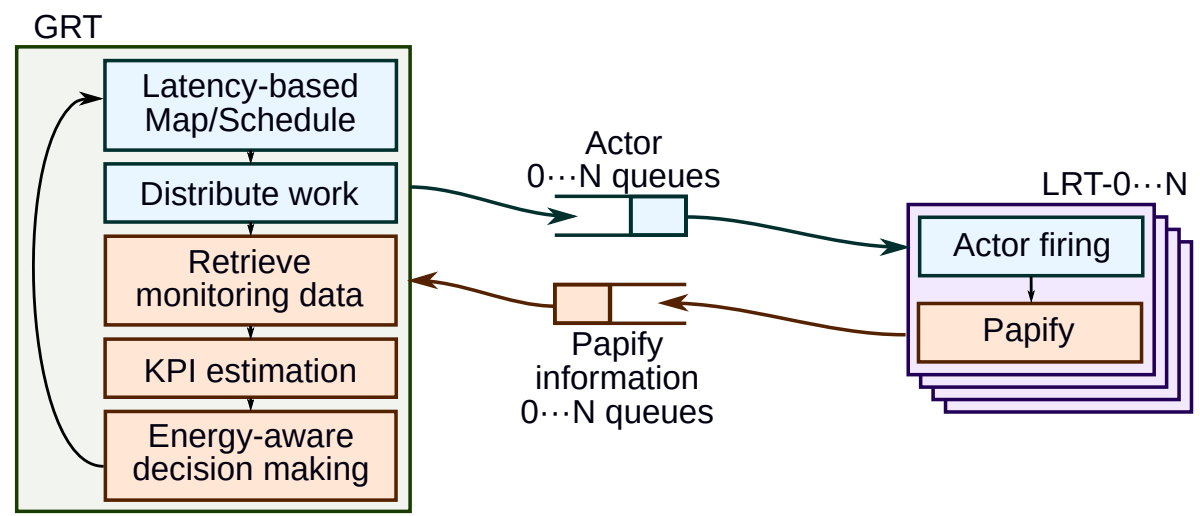

Figure 6-12: SPiDER runtime structure including energy-awareness feedback loop 
During the first stage, monitoring, the integration of both PAPIFY and SPiDER explained in Section 4.3 is used. This integration provides SPiDER with monitoring capabilities that allow developers to enable the application characterization in terms of timing and PAPI events. This information is independent for each actor. In this case, the monitoring configurations associated to each actor-PE pair are provided to both GRT and LRTs. In turn, every time one actor is executed, its monitoring information -PAPIFY information- is sent back to the GRT, as shown in Figure 6-12.

After the execution of each iteration of the application graph, all the monitoring data is retrieved so as to launch the KPI estimation step. During this stage, the execution time associated to each actor-PE pair is updated within the GRT information. Additionally, energy estimation is performed using the PAPI events retrieved during the monitoring. To do so, again, the energy consumption model associated to the computation developed in Section 5.6 is employed. In order to provide SPiDER GRT with the energy consumption models, during the automatic code generation, these models are uploaded as part of SPiDER configuration information. As a result, the timing information is updated so as to enhance the latency-based mapping/scheduling algorithm, while the energy consumption of each actor is used when evaluating the system configuration if the energy-awareness is enabled.

At this point, SPiDER can implement an energy-aware decision making step. To do so, each application deployment is evaluated and, if necessary, a new system configuration is proposed. This evaluation considers two indicators: performance reached and energy consumption. Regarding the performance, the time per iteration $\left(T_{\text {iter }}\right)$ is measured and the reached performance is estimated using Equation 6-2. Once the performance is obtained, the total energy consumption is computed considering 1 ) the number of active PEs (resource-active consumption Section 5.4), 2) the communications included in the implementation (communication consumption - Section 5.5) and 3) the energy consumed by the application being executed Performance times per second (computation consumption - Section 5.6.

$$
\text { Performance }\left[\frac{\text { Executions }}{\mathrm{s}}\right]=\frac{1}{T_{\text {iter }}}
$$

After evaluating the current system configuration, the decision is taken following the energy-aware tuning algorithm proposed in Section 6.2. In the case of the runtime optimization loop, one different system configuration is tested until the optimum is found. That is, once the searching finishes, the best configuration among the tested ones, which will be the one with the lowest energy consumption reaching the performance objective, is selected.

Once the basic energy-aware decisions have been explained, in order to consider the dynamic behavior supported bySPiDER, a dependency to the dynamic parameters has been included. As explained in Chapter 3 , these parameters modify the application dataflow graph and, consequently, its implementations also vary, as the application itself may change. To include this variability in the scope of the energy-awareness mechanism, the searching is linked to the application dynamic parameters. By doing 
so, if one of the parameters changes at runtime, the searching will either: 1) start from scratch if the dynamic parameter combination is used for the first time or 2) restart from the status stored when the dynamic parameters changed if the dynamic parameter combination has been used before. As a result, with this procedure, if the optimization process for one set of dynamic parameters has already finished, if they are selected again, the best configuration will be directly retrieved, thus, saving time and adapting the system in one iteration.

Finally, considering that one system requirement is to surpass a performance objective, a failsafe protocol has been included. This protocol takes advantage of the self-awareness implemented in SPiDER runtime resource manager and monitors that the minimum performance is reached. This may not be true in systems running an OS, as it may interfere with the application execution. To deal with this problematic, once the optimization searching finishes, the failsafe is enabled. It consists on a continuous checking of the performance achieved in every iteration, where the number of active $\mathrm{PE} s$ is increased by one if this performance objective is not reached during 10 iterations in a row. Consequently, thanks to this approach, SPiDER is able to react to system anomalies and tries to ensure the fulfilling of the requirements.

\subsection{Discussion}

This chapter has presented the energy-aware tuning approach proposed as one of the original contributions of this $\mathrm{PhD}$. This mechanism can be applied to tune latency-based mapping/scheduling algorithms to take energy-aware decisions when optimizing the system in an iterative way. Specifically, an energy-aware optimization loop has been built considering the monitoring infrastructure presented in Chapter 4 . the energy estimations that can be estimated using the model developed in Chapter 5 and the energy-aware decision making explained in this chapter.

In order to validate this energy-aware optimization loop, it has been implemented in two frameworks. The former focuses on including energy-awareness in the DSE procedure of PREESM. The latter has been embedded within SPiDER runtime to perform on-the-fly energy-aware system optimizations.

These two implementations have been used to optimize the energy consumption of the HSI cancer detection system considering different performance/energy consumption trade-offs. The results associated to this analysis are detailed in Chapter 7 . The energy-awareness at design time is thoroughly analyzed in Subsection 7.3.4, while its runtime counterpart is characterized in Subsection 7.4.3. 



\section{RESULTS}

This chapter gathers all the experimental results associated to the development of the HSI cancer detection application on the MPPA platform, together with those obtained while validating the design methodology proposed during this $\mathrm{PhD}$. Specifically, the setup used to extract these results is explained in Section 7.1.

Additionally, this chapter is divided into 3 different sections, each of them corresponding to the type of design methodology used. First, the implementation performed by hand, which will be used as a baseline to compare with the automatically optimized implementations, is explained in Section 7.2. Secondly, the implementation obtained when using the design time optimization loop implemented in PREESM is detailed in Section 7.3. Finally, the results associated to the implementation using the runtime optimization loop embedded within SPiDER are analyzed in Section 7.4.

It should be noted that the results obtained with both design (PREESM) and runtime (SPiDER) optimization loops are divided into two studies: latency-based and energy-aware. The former employs the loop to optimize latency, retrieving only timing information. These results are compared, in terms of performance, with the ones obtained with PREESM and SPiDER when no timing data is considered. The latter uses the complete energy-aware optimization loop to perform multi-objective optimizations (latency and energy), gathering both timing and energy consumption information. In this case, the results are compared, in terms of performance and energy consumption, with the ones obtained with the implementation done by hand.

\subsection{Setup}

During this section, the setup used to extract the results of this chapter is explained. Specifically, first, details about the HSI cancer detection application and the MPPA architecture configurations are provided. After this, the experiments associated to the functional validation, which will be run using the implementation done by hand, are outlined. Then, the specific configuration and experiments used to validate the energy-aware optimization loop in both design time and runtime are detailed. 


\section{Application and architecture}

On the application side, 6 images have been selected from the database to carry out the study on the MPPA. Specifically, as shown in Table 7-1, there are 3 human brain images and 3 human skin ones. As can be observed in the table, the images have different spatial resolutions (Rows and Columns values in the table) and different spectral resolution too (Bands values). Specifically, brain images have a total amount of 128 hyperspectral bands, while skin images have 100 bands. This variability has been included so as to analyze the impact of both spatial and spectral resolution in the achieved performance. Additionally, the size of each image, i.e., its memory footprint, is included to highlight the amount of data that needs to be analyzed in each case. This

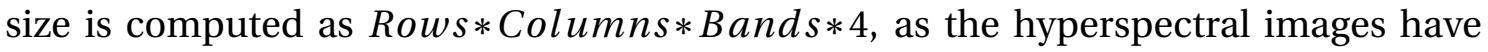
been stored using float data type, i.e., 4 bytes per value.

It is worth pointing out that all the experiments that will be performed discern among 4 different classes: healthy tissue, tumorous tissue, hyper-vascularized tissue and background/others.

Table 7-1: HSI cancer images used during the validation

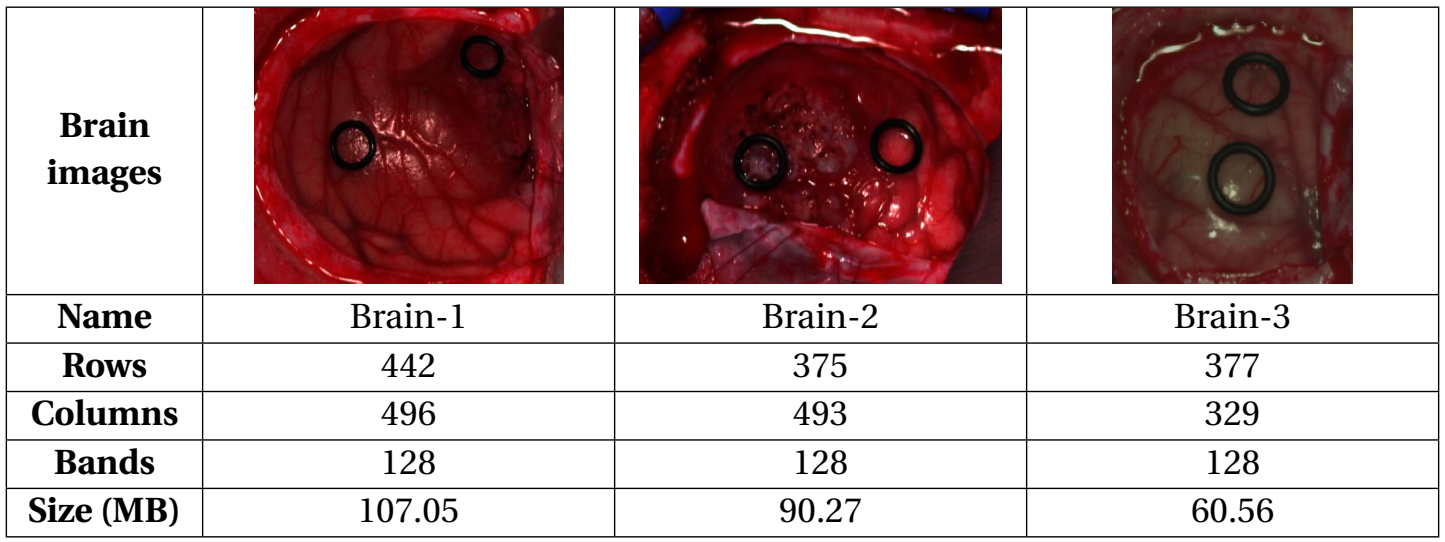

\begin{tabular}{|c|c|c|c|}
\hline $\begin{array}{c}\text { Skin } \\
\text { images }\end{array}$ & & & \\
& & & \\
\hline Name & Skin-1 & Skin-2 & Skin-3 \\
\hline Rows & 1000 & 1000 & 1000 \\
\hline Columns & 1000 & 1000 & 1000 \\
\hline Bands & 100 & 100 & 100 \\
\hline Size (MB) & 381.47 & 381.47 & 381.47 \\
\hline
\end{tabular}

Concerning the MPPA architecture, a frequency of $550 \mathrm{MHz}$ has been selected, as it is the value used to develop the energy consumption model (Section 5.6). 


\section{Functional validation}

In the case of the functional validation Section 7.2), the number of MPPA resources used $(\mathrm{CC} s$ and $\overline{\mathrm{PE}} \mathrm{s})$ is modified when evaluating the implementation. In this case, the configurations are gathered in Table 7-2, together with a small description of the experiment. Specifically, inter-cluster parallelization refers to distributing the workload among the $16 \mathrm{CC}$, whilst intra-cluster parallelization means that the workload associated to each $\mathrm{CC}$ is distributed among its active $\mathrm{PE}$.

Table 7-2: MPPA configurations used during the functional validation

\begin{tabular}{|c|c|c|c|}
\hline Name & $\mathbf{C C}$ & PEs per $C \mathrm{CC}$ & Short Description \\
\hline Seq & 1 & 1 & Sequential execution on one PE of one CC \\
\hline 1-CC & 1 & 16 & $\begin{array}{l}\text { All the } \mathrm{PE} \text { within a } \mathrm{CC} \text { are used } \\
\text { Intra-cluster parallelization enabled }\end{array}$ \\
\hline 1-PE & 16 & 1 & $\begin{array}{c}\text { One } \mathrm{PE} \text { of each } \mathrm{CC} \text { is used } \\
\text { Inter-cluster parallelization enabled }\end{array}$ \\
\hline MPPA & 16 & 16 & $\begin{array}{c}\text { The whole MPPA is used } \\
\text { Inter- and intra-cluster parallelization enabled }\end{array}$ \\
\hline
\end{tabular}

\section{Energy-aware optimization loops}

Regarding the validation of the Y-chart dataflow-based energy-aware design methodology, instead of fixing the active MPPA resources, different working modes are considered. These working modes, as shown in Table 7-3, have different performance requirements depending on the use-case to which they are applied, i.e., Brain or Skin. Specifically, 3 working modes have been created: Fast, Normal and Slow.

In the case of Brain modes, that is, in the neurosurgical field of application, the requirements are directly linked to the HELICoiD project ones. In this regard, HELICoiD main constraint is the processing time of the hyperspectral images, while energy is not part of the requirements. Likewise, as explained in [Fabelo'18b], the supervised classification algorithm, which is the one being accelerated on the MPPA, is executed in parallel with the unsupervised part of the algorithm. In other words, the time available to execute the supervised classification block is the one taken by the counterpart computer to execute the unsupervised algorithm, which, in [Fabelo'18b], has a minimum value around 20s. Nevertheless, further improvements of the unsupervised part of the algorithm can be easily foreseen, since, in HELICoiD, this part has not been parallelized nor optimized. Taking into account this possible improvement, the processing time (performance) associated to the 3 Brain working modes are 10s (0.1 images/s), 15s (0.066 images/s) and 20s (0.05 images/s) for Fast, Normal and Slow modes, respectively.

From the dermatological point of view, and considering the utilization of a batterysupplied device, the timing constraints are relaxed whilst the energy limitations grow. In this field, Skin, the system should be able to provide results during the medical 
appointment. For that reason, the processing time (performance) associated to these working modes have been adapted, as an example, to 60s (0.017 images/s), 90s (0.011 images/s) and 120s (0.008 images/s), for Fast, Normal and Slow modes, respectively. By doing so, the Skin field of application will also cover different situations in which the processing time and the energy consumption are balanced, while providing the results during the medical appointment.

Table 7-3: Working modes used in the Y-chart dataflow-based energy-aware design methodology experiments

\begin{tabular}{|c|c|c|c|c|}
\hline \multirow{2}{*}{ Field } & $\begin{array}{c}\text { Working } \\
\text { mode }\end{array}$ & $\begin{array}{c}\text { Processing } \\
\text { Time (s) }\end{array}$ & $\begin{array}{c}\text { Performance } \\
\text { (images } \\
\text { per second) }\end{array}$ & Short Description \\
\hline \multirow{3}{*}{ Brain } & Fast & 10 & 0.1 & - Tight performance constraints \\
\cline { 2 - 4 } & Normal & 15 & 0.066 & - Plugged to the grid \\
\cline { 2 - 4 } & Slow & 20 & 0.05 & - No energy constraints \\
\hline \multirow{3}{*}{ Skin } & Fast & 60 & 0.017 & - Loose performance constraints \\
\cline { 2 - 4 } & Normal & 90 & 0.011 & - Battery supplied \\
\cline { 2 - 4 } & Slow & 120 & 0.008 & - Energy constrained \\
\hline
\end{tabular}

Summing up, a total amount of 18 experiments, where either the hyperspectral image or the working mode vary, are considered so as to validate the iterative energyaware design of dataflow applications. These experiments will be named hereafter as shown in Table 7-4.

Table 7-4: Validation experiment terminology

\begin{tabular}{|cc|c|c|c|}
\hline Image & Working mode & Slow & Normal & Fast \\
\hline & & & & \\
\hline Brain-1 & Brain-1-S & Brain-1-N & Brain-1-F \\
\hline Brain-3 & Brain-2-S & Brain-2-N & Brain-2-F \\
\hline Skin-1 & Brain-3-S & Brain-3-N & Brain-3-F \\
\hline Skin-2 & Skin-1-S & Skin-1-N & Skin-1-F \\
\hline Skin-3 & Skin-2-S & Skin-2-N & Skin-2-F \\
\hline & Skin-3-S & Skin-3-N & Skin-3-F \\
\hline
\end{tabular}

It is worth pointing out that, as introduced in Section 6.1, the scope of this $\mathrm{PhD}$ is a system running a streaming application that cannot go idle after finishing the processing. In this regard, streaming applications are characterized by running iteratively; hence, minimizing the average power consumption implies minimizing the energy consumption ratio (Energy $=$ Power $*$ Time). Figure 7-1 graphically represents this equivalence, where Power 1 is linked to Energy 1, and reducing the power consumption to Power 2 implies reducing the energy consumption ratio to Energy 2. Consequently, during the analysis of the results, power/energy consumption optimization will be presented as power/energy savings. 


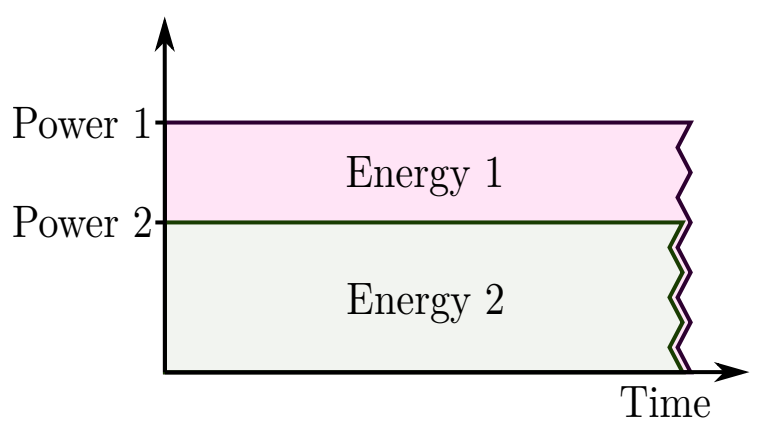

Figure 7-1: Power and energy reduction equivalence for an infinite time

\section{Design time optimization loop}

In the case of the design time loop (Section 7.3), first, PREESM optimization loop will be used to enhance the dataflow implementation in terms of latency and compared with the system generated when no timing information is included. After this, this implementation will be characterized in terms of energy consumption using PAPIFY and, then, the profiling will be included within the scenario file, as explained in Section 6.3. Finally, energy-awareness will be enabled during the DSE to generate the system using the 3 working modes explained before, and the obtained implementations will be compared with the implementation done by hand.

\section{Runtime optimization loop}

Finally, in the case of the runtime loop (Section 7.4), the same approach is considered. The first set of experiments focuses on addressing latency-based optimization with and without PAPIFY providing feedback to SPiDER. First, standard SPiDER -without timing information- will perform both the mappings and the scheduling with the objective of optimizing latency. Secondly, PAPIFY will be enabled and will feed SPiDER GRT with timing information to optimize the mappings and scheduling that it can perform. The second set of experiments, in turn, will validate the energy-awareness runtime optimization loop that has been integrated within SPiDER.

Considering that, as explained in Subsection 3.3.2, the MPPA architecture is not supported by the SPiDER backend, the validation of the energy-awareness runtime optimization loop is carried out in two steps. First, the part of the optimization loop that has been implemented in the architecture-independent part of SPiDER is evaluated onto a 16-core x86 architecture using a simulated energy consumption model. After this, the actual energy consumption model of the MPPA architecture developed in Chapter 5, which is the only part of the optimization loop that is architecture dependent, is validated when executing the HSI cancer detection application using different MPPA configurations.

Specifically, in the first part of the validation, the simulated energy consumption model used will be based on the one extracted for the MPPA in Chapter 5. The simulated model has the following characteristics: 
- Resource-active consumption: each x86 core is simulated using the resourceactive consumption of one CC of the MPPA

- Communication contribution: simulated as inter-cluster communication $\left(S M E M_{R}\right.$ and $S M E M_{W}$ are enabled).

- Computation contribution: linked to the available PAPI events of the x86 whose functionalities are closer to those from the MPPA.

__PAPI_L1_ICH substitutes_K1_IHITS. This event also gathers the instruction $\mathrm{Ll}$ cache hits.

- perf::L1-DCACHE-LOAD-MISSES substitutes_K1_DMISS. This native event counts the misses when loading data from the L1 cache.

__PAPI_STL_ICY substitutes_K1_DMISS_STALLS. Contrary to the MPPA PAPI event, where the cycles stalling after a cache miss are accounted,_PAPI_STL_ICY accumulates the cycles without executing any instruction. This event is the closest one, in terms of functionality, among those available on the target architecture.

- _PAPI_TOT_INS substitutes_K1_EXEC_BUNDLES. Although the MPPA event counts the bundles that are executed by the processor, this type of monitor is not available in the target architecture. Nevertheless, _PAPI_TOT_INS is selected, as it is linked to the total number of instructions executed by the processor, which is the closest event available on the target platform.

After this, during the validation of the real energy consumption model, the HSI cancer detection application will be deployed onto the MPPA using a large set of configurations. For each configuration, the energy consumption will be both measured and estimated so as to validate the energy consumption model developed in Chapter 5. In this study, the architecture-dependent block of the runtime energyaware optimization loop is characterized in terms of accuracy.

By doing this two-step validation, both the energy-aware optimization loop implemented within SPiDER and the energy estimation for the MPPA architecture are evaluated. As a result, it can be easily foreseen that the combination of both can lead to a self-adaptation loop where the energy consumption is minimized using SPiDER on the MPPA. As will be explained in Section 8.4, the support of SPiDER on the MPPA has been considered as future work. The reason why it has not been included within this $\mathrm{PhD}$ is the imminent release of a new version of SPiDER that would make the integration obsolete. Consequently, once the new version of SPiDER is released, as the optimization loop has been mainly embedded within the GRT, which is architecture independent, implementing it onto the MPPA will be straightforward. 


\subsection{Functional validation}

This section analyzes the results obtained when manually implementing the HSI cancer detection algorithm onto the MPPA architecture. In this case, no energy constraints are considered, as this implementation aims at performing a functional validation of the algorithm when optimizing latency for both brain and skin images. In order to provide a deep understanding of the obtained results, first a detailed description of the implementation of each stage of the processing chain is presented in Subsection 7.2.1. After this, Subsection 7.2.2 presents the results obtained through the evaluation in terms of processing time using the different images and MPPA configurations presented in Section 7.1.

\subsubsection{Implementation by hand}

In order to carry out the implementation, as explained in Subsection 3.1.1, it should be considered that hyperspectral images gather a huge amount of information and, thus, they take up a lot of memory. Furthermore, each MPPACC has a total amount of $2 \mathrm{MB}$ of SMEM to store the OS, the application code and the application data. This means that, in practice, only $1 \mathrm{MB}$ is available to store data in each $\mathrm{CC}$.

As a result, the implementation of the HSI processing chain on the MPPA platform focuses on exploiting the intrinsic parallelism of the application and, when possible, splitting the image processing among as many $\mathrm{PE} s$ as possible to minimize the latency. Additionally, as explained in Subsection 3.1.2, the asynchronous communication protocol provided by Kalray is used to optimize the data management.

Nevertheless, as the processing chain used in this $\mathrm{PhD}$ to process hyperspectral images is, as shown in Figure 3-2, composed of 3 different algorithms, they present different parallelism natures. As a consequence, each part of the algorithm has been analyzed independently so as to extract the workload distribution that better fits the MPPA constraints. It is worth pointing out that, in the implementation, data is first stored in the IO subsystem; then, the CCs read and write from/to $\mathrm{IO}$ so as to synchronize and share information among the processing resources.

To lead the detailed explanation of the implementation done by hand, Figure 7-3 represents a block diagram of the execution workflow. The timings appearing in this figure are used to exemplify a possible application execution. In this figure, both $\mathrm{IO}$ and CCs cooperate to execute the HSI cancer detection algorithm. Additionally, two extra blocks have been included, namely Transpose and FeatureMatrix Building. The former transposes the input image before executing the SVM block. The latter builds the input used during KNN searching. During the rest of the subsection, both the functionality and the implementation of each block are thoroughly analyzed. 


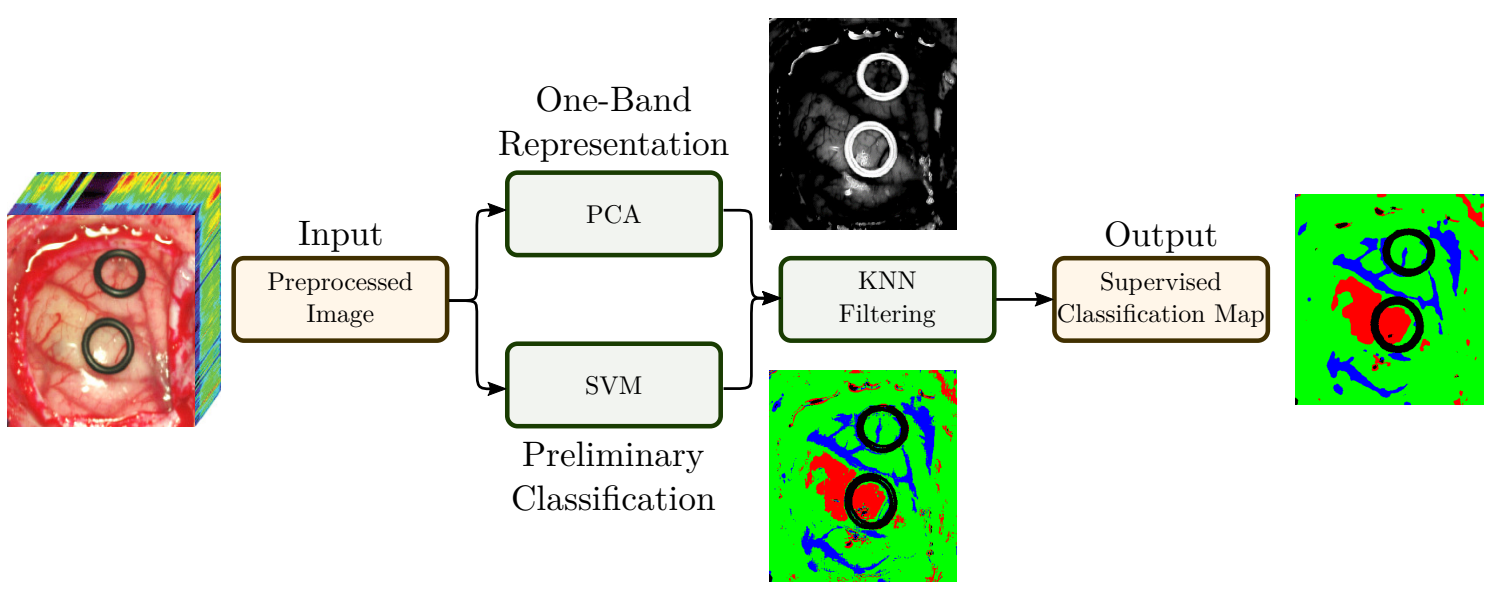

Figure 3-2: HELICoiD processing chain. Supervised classification step (repeated from page 37

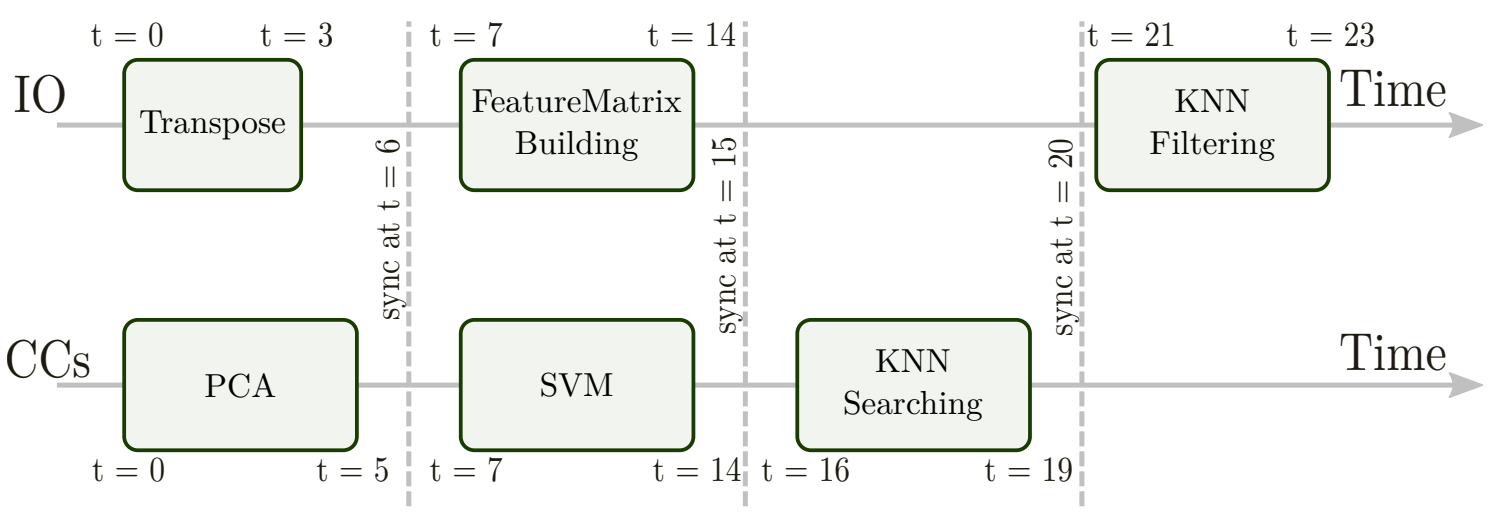

Figure 7-3: Workload distribution proposed to implement by hand the HELICoiD processing chain on the MPPA

\section{PCA}

In the case of PCA each step composing the algorithm has been analyzed independently so as to optimize the parallelization of each part. These steps are: image centering, covariance, diagonalization and projection.

Image centering step computes the mean value of each band and, after that, subtracts this value from the band itself. As a consequence, this part of the PCA algorithm can be considered band-wise parallelizable, which means that each band of the hyperspectral image can be processed in parallel. In order to exploit this parallelism, the hyperspectral image bands are divided into blocks of $16,384(16 * 1,024)$ pixels. Each band block will, then, have a fixed size of $64 \mathrm{kB}(16 * 1,024 * 4)$, as the images are stored using float data types (4 bytes). Using this block size, in the case where 16 $\mathrm{PE} s$ are enabled within the $\mathrm{CC}$ and considering that each $\mathrm{PE}$ can upload its own block, the 1MB of SMEM available for data storage will be used.

In this context, to reuse data during the image centering step, the processing of one hyperspectral band is associated to one specific CC. As a result, the implementation follows Algorithm 7-1, which is also detailed in [Lazcano'19b]. First, each enabled 
$\mathrm{PE}$ within the $\mathrm{CC}$ reads a block of the hyperspectral band from the $\mathrm{IO}$ (line 5) and, once it has been written in the SMEM, all the values are added up (line 6). If there are more than 16 blocks for each band, which happens for images with more than 262,144 pixels $(16 * 16 * 1,024)$, this process is iteratively repeated until all the values composing the hyperspectral band are accumulated (lines 4 to 7 ). After this, the average is computed by dividing the accumulation by the total number of pixels composing the hyperspectral image (line 8). Then, each $\mathrm{PE}$, again, uploads band blocks iteratively (lines 10 to 15) so as to subtract the computed average from the original hyperspectral band (line 12). Finally, the result of each block is sent back to the IO. Specifically, data is written to the IO in both the standard (line 13) and transposed (line 14) orders so as to have it properly ordered for covariance and projection steps, respectively.

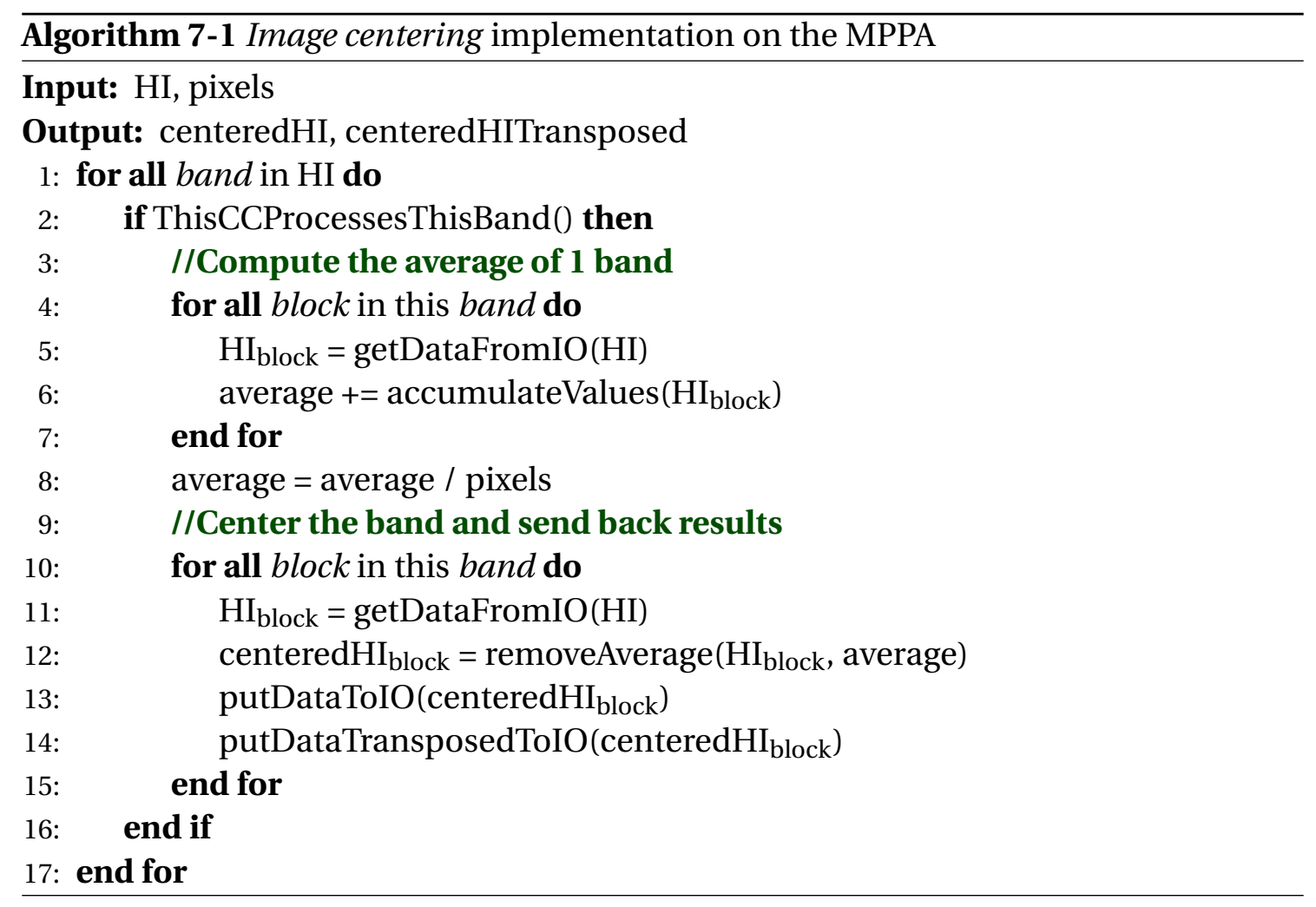

Secondly, in the case of covariance computation step, it consists in multiplying the centered image matrix by its transpose to obtain the so-called covariance matrix. Specifically, each value composing the covariance matrix is computed as a vector multiplication of 2 hyperspectral bands. In this line, this implementation has been optimized considering that a matrix multiplied by its transposed results in a symmetric matrix. Consequently, the number of transmissions and the workload itself are almost reduced by half. Additionally, in order to implement the 2-band vector multiplication on the MPPA|CC $\$$, each band has been divided, again, in blocks of 16,384 pixels.

The implementation of the covariance computation is the one represented in Algorithm 7-2, In this algorithm, the processing of each row of the covariance matrix is linked to one $\mathrm{CC}$ (line 2), while each value belonging to that row is computed by 
one specific $\mathrm{PE}$ (line 8). As a result, for each row, the band blocks are iteratively read from the IO (line 5); and, as an inner loop, the column band blocks are also iteratively uploaded (line 11). Once the CC has both available, the multiplication of row-bycolumn bands is performed (line 12). Once all the values of one row of the covariance matrix are computed, the results are sent back to the $\mathrm{IO}$ in both the standard (line 18) and transposed (line 19) orders. These two transmissions are required to fill the whole covariance matrix when using the aforementioned simplification.

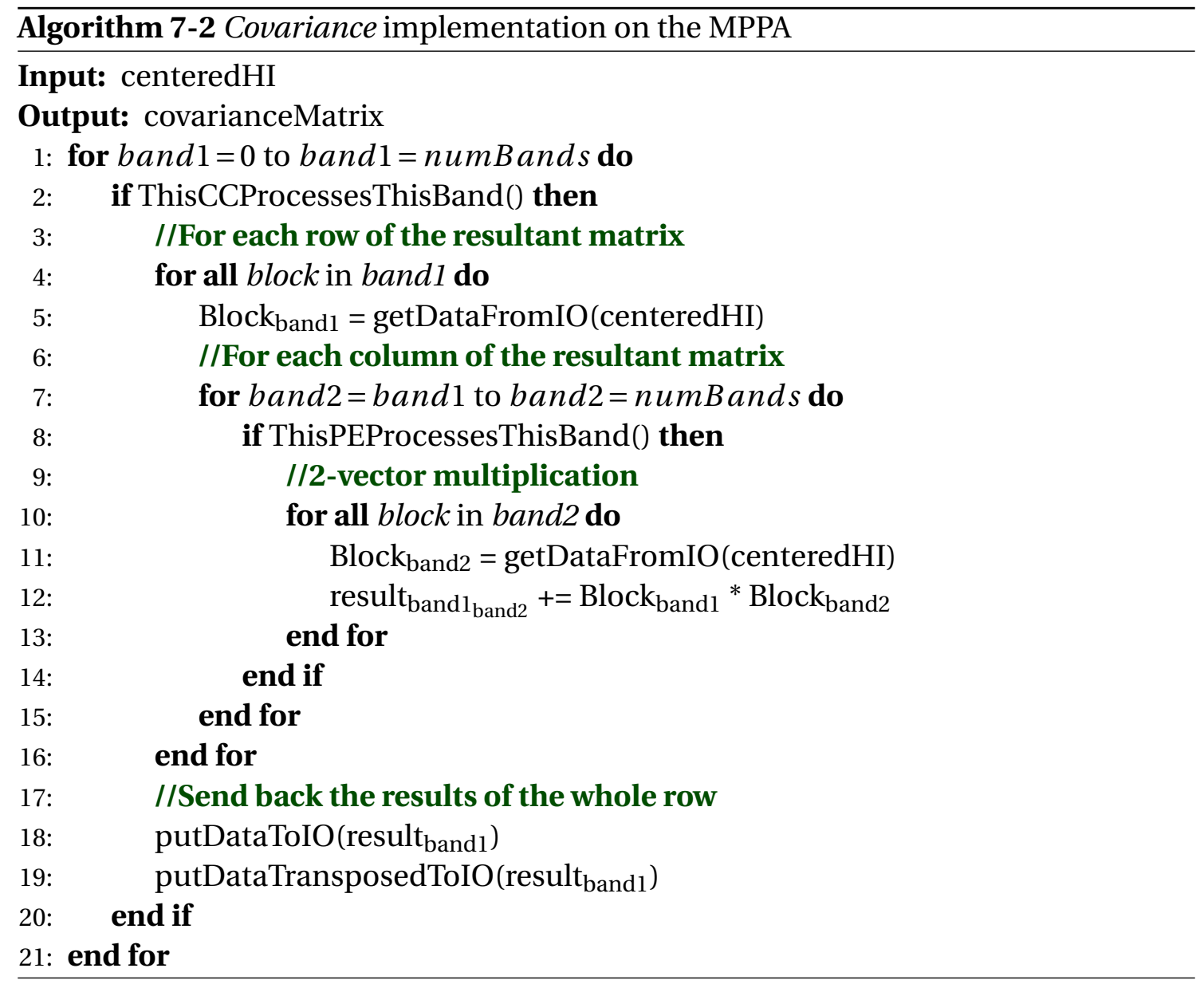

Once the covariance matrix is obtained, next step is its diagonalization. As explained in [Lazcano'17c], Jacobi algorithm has been selected to perform this task. The rationale behind this decision is that this procedure can be parallelized and, thus, the time required to diagonalize the matrix can be minimized. Specifically, this algorithm consists of an iterative procedure where a set of elements of the matrix are selected as candidates to be zeroed. For each element, in turn, the eigenvector matrix is updated. As a result, once the matrix has been diagonalized, i.e, all its nondiagonal elements are zeroed, both the eigenvectors and the associated eigenvalues (the diagonal of the diagonalized matrix) are obtained. Regarding the implementation, as the input matrix to be used is just composed of bands*bands values, it perfectly fits within the CC memory. Consequently, in order to save memory transmissions, only 
one CC will be in charge of computing the Jacobi algorithm, since the synchronization among CCs working in parallel would overcome any potential speedup.

As a result, Jacobi algorithm has been implemented onto the MPPA following Algorithm 7-3. As can be seen, the first step is to read from the IO the covariance matrix previously computed (line 2). Once it is uploaded, the diagonalization procedure starts: 1) the values to be zeroed are selected by one of the PEs (line 5) and, for each value, 2) the rows of the diagonal matrix, together with the eigenvector matrix, are updated (line 8) and 3) the columns of both matrices are also updated (line 9). By doing this separation when updating the rows and the columns, each value being zeroed can be processed in parallel. Finally, the eigenvectors are ordered considering the value of their associated eigenvalues (lines 13 to 15). This ordering is required to avoid losing relevant information during PCA since the hyperspectral images are projected onto the eigenvectors that contain most of the information, which are the ones with the highest eigenvalues.

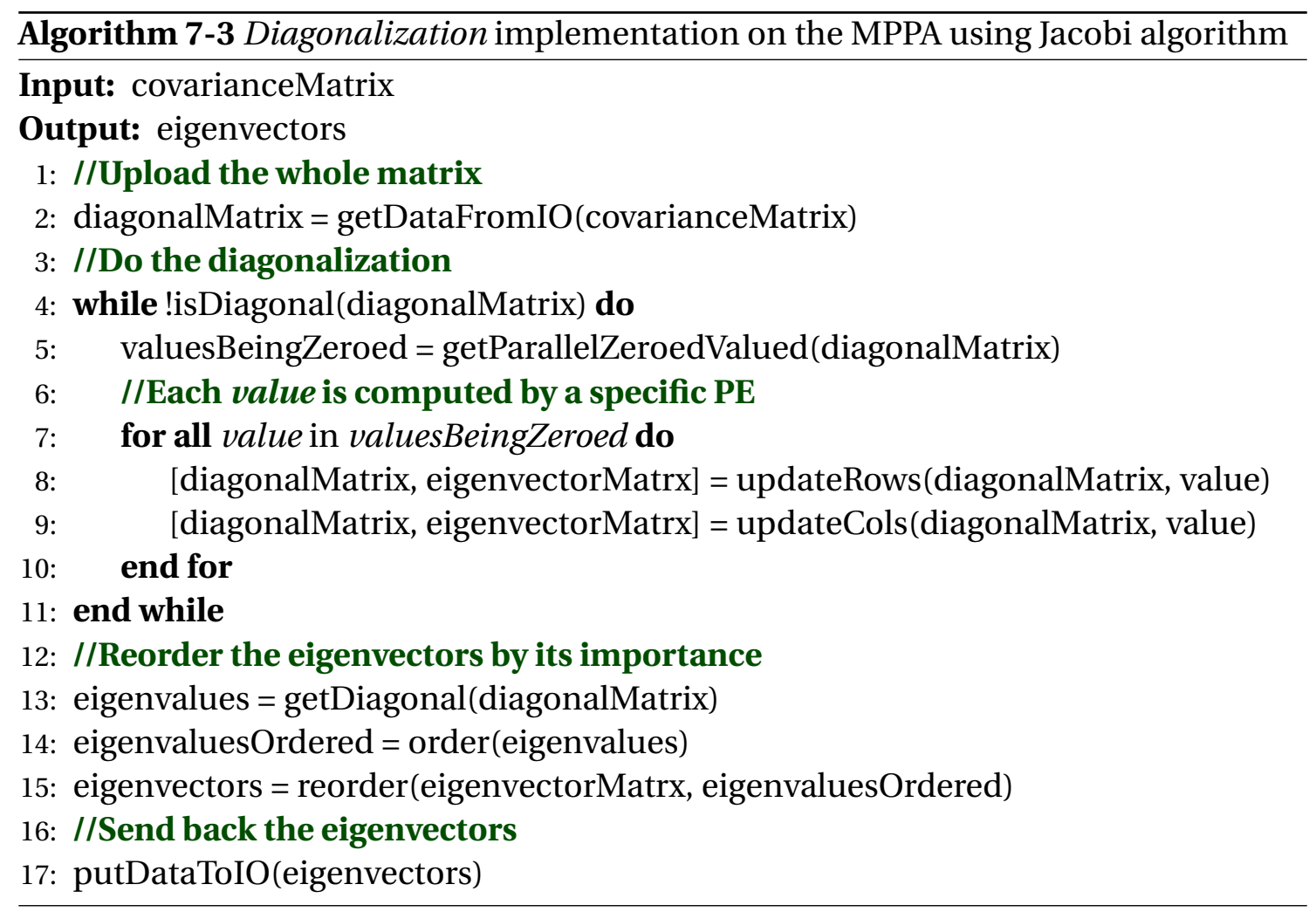

Finally, the last step of $\mathrm{PCA}$ is the projection of the centered image onto the eigenvectors obtained during the diagonalization stage. In this step, depending on the principal components required, the projection is carried out using a different number of eigenvectors. In this case, as the number of principal components to extract is 1 (1band representation of the image), only 1 eigenvector has to be used. Additionally, considering that the whole image has to be projected onto that eigenvector, the centered image has to be divided in blocks. To perform this division, it has been considered that each pixel can be projected independently from the rest of the image 
and, thus, blocks of pixels can be used. Finally, as one pixel is composed by up to 128 hyperspectral bands, its maximum size would be 512B $(128 * 4)$. As a result, blocks of 64 pixels $(32 \mathrm{kB})$ are built, which means that when all the $\mathrm{PE} s$ inside a CC are active, $512 \mathrm{kB}$ are filled with data.

In this line, the implementation of the projection stage of the PCA algorithm follows Algorithm 7-4. In this implementation, first, the eigenvector that will be used to project the image is loaded (line 2) in each CC. After this, the blocks of pixels (pixelBlock) are iteratively uploaded by each active $\mathrm{PE}$ within each active $\mathrm{CC}$ (line 6) and each pixel is projected onto the eigenvector using a dot product (line 8) to obtain the first principal component $(P C)$. Finally, this $P C$ is sent back to the $\mathrm{IO}$ (line 10) to build the 1-band representation of the hyperspectral image, which is used as an input of the KNN block.

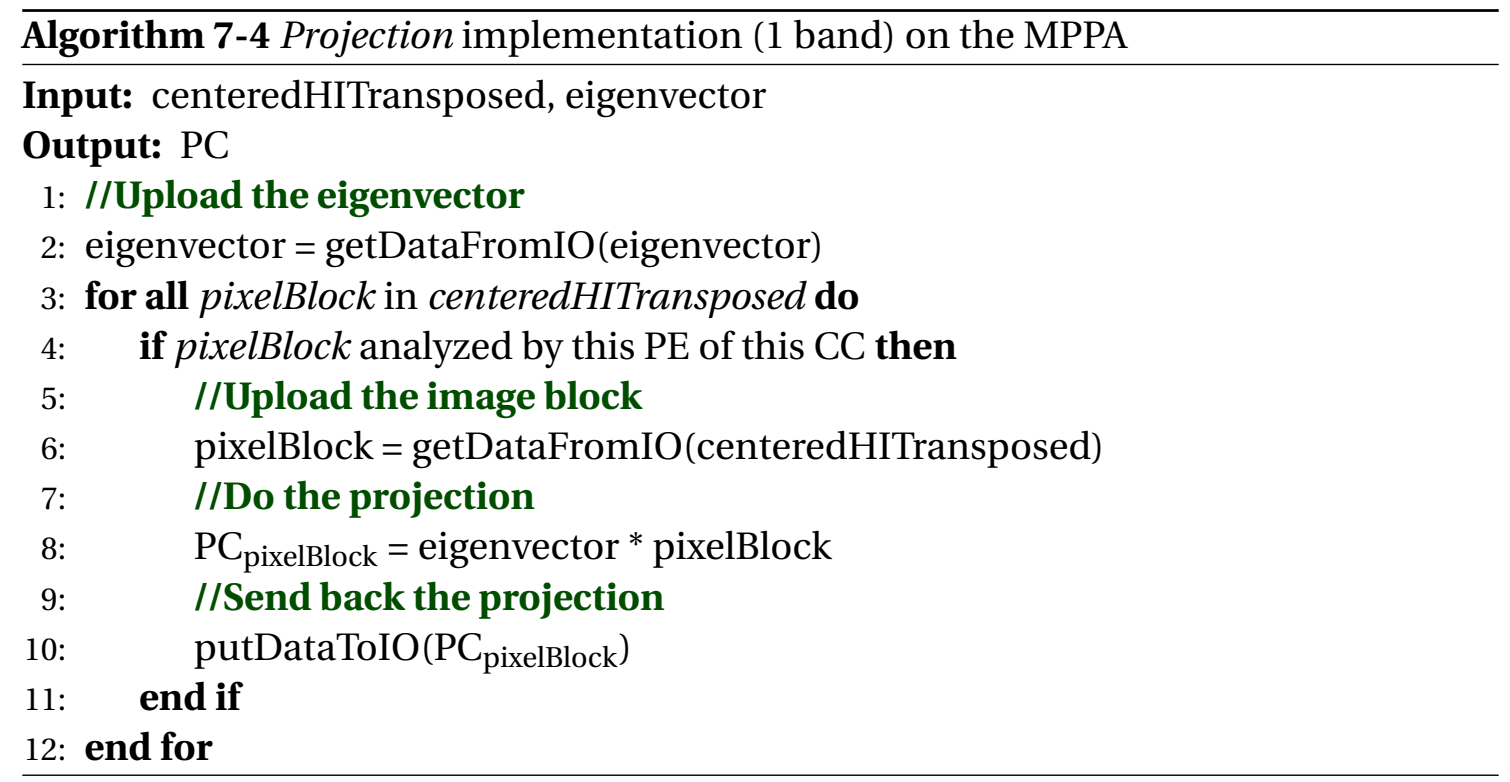

\section{SVM}

Once the implementation of PCA algorithm onto the MPPA has been explained, the implementation of SVM is addressed. Contrary to PCA, SVM algorithm can be executed using a pixel-wise parallelization strategy during the whole processing. Consequently, the input hyperspectral image needs to be transposed before starting the SVM. As this algorithm is completely independent from the execution of PCA, this matrix transposition is carried out in the IO, while the CCs are executing the $\mathrm{PCA}$ itself.

Taking advantage of the pixel-wise parallelization of SVM, the algorithm has been implemented as a single step where the hyperspectral image is divided in blocks of 64 pixels (maximum block size of $32 \mathrm{kB}$ ) and each block is processed independently. As a result, the communication between $\mathrm{IO}$ and $\mathrm{CC} s$ is minimized, since only one transmission in each direction is required per pixel block.

The implementation of the SVM algorithm onto the MPPA architecture follows Algorithm 7-5, which is also explained in [Lazcano'19b]. The different steps are deeply 
detailed in [Madroñal'17b], where the minimization problem is solved following the work presented in [Wu'04]. As can be seen, the first step is to upload the SVM classification model (line 2), which has been previously extracted off-line. After this, each block of the image is iteratively uploaded (line 6) by a different PE of each CC and 3 steps are carried out. First, the distances (pixelDistances) of the pixel to the different model hyperplanes ( model $_{\text {hyperplanes }}$ ) composing the classification model are computed (line 8). These hyperplanes are obtained during the training phase of the machine learning algorithm and represent the frontiers that maximize the distance between each pair of classes. Secondly, the pixel binary probabilities (binaryProbs) of belonging to each of the 2 classes separated by the hyperplane are obtained (line 10). To do so, the classification models have been enhanced with two values per hyperplane ( model $_{\text {probs }}$ ) that define a sigmoid function to weigh the distance to the hyperplane and provide the probability to belong to each of the two classes under study. Finally, the third step consists in a minimization problem that combines the binaryProbs so as to obtain the probability of a pixel to belong to each class under study when all the classes are considered (pixelsProbabilities) (line 12). Finally, data is sent back to the $\mathrm{IO}$ subsystem so as to compose again the whole image and start the processing of the KNN part of the algorithm.

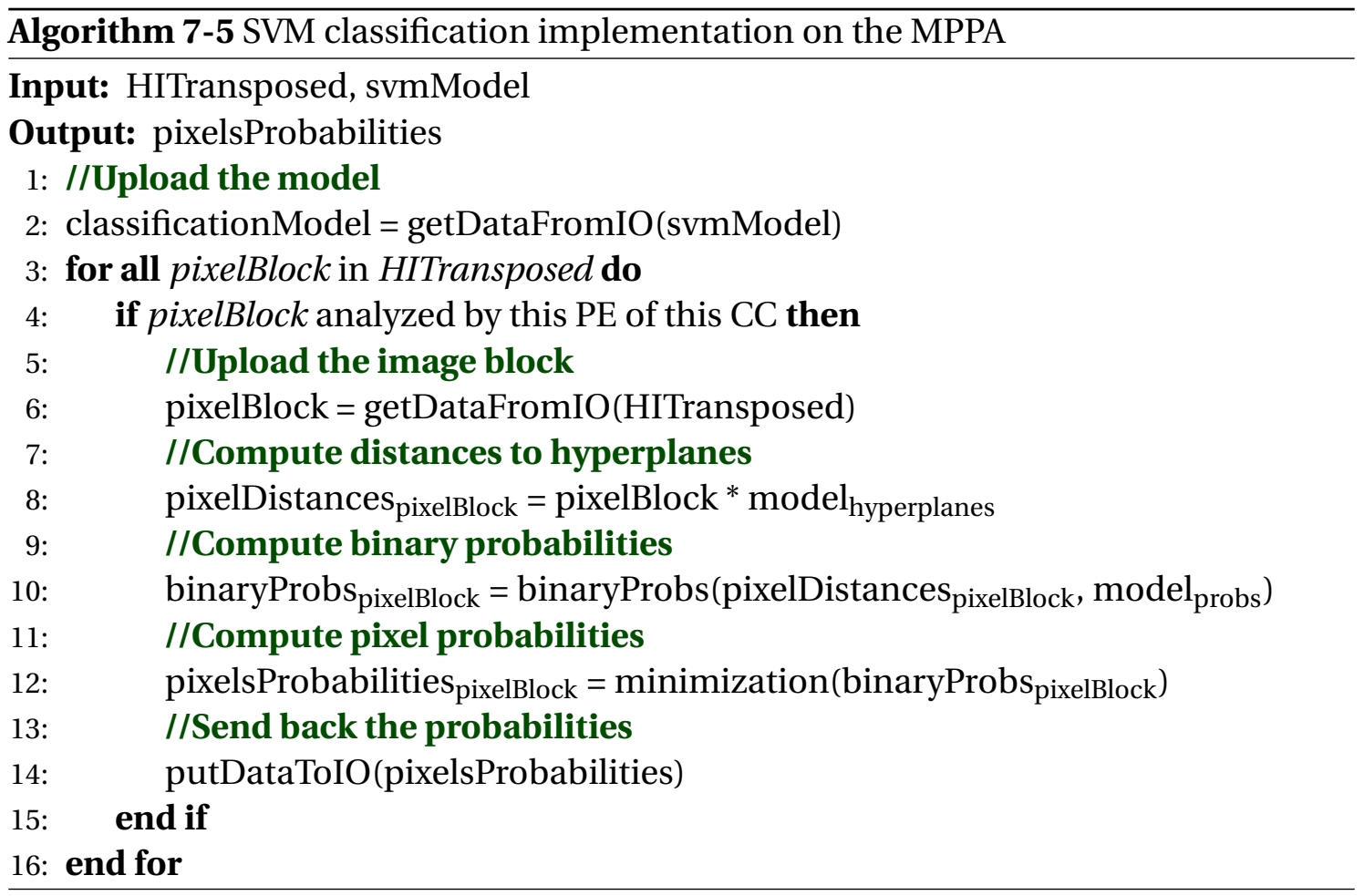




\section{KNN}

Finally, the last part of the hyperspectral processing chain that is being accelerated corresponds to the $\mathrm{KNN}$ filtering. This algorithm consists of 3 different stages: 1) neighbor searching, 2) filtering and (optionally) 3) class selection. Consequently, again, each part of the algorithm is analyzed independently so as to select the best parallelization strategy in each case.

In the case of neighbor searching step, the inputs to be used are the principal component -the so-called PC extracted during PCA algorithm (Algorithm 7-4) - and the spatial coordinates of each pixel within the hyperspectral image. In this particular case, it has been considered that the distance among pixels will be computed following Equation 7-1. This equation is a particular case of Equation 3-1, where both spatial and spectral information are equally balanced $(\lambda=1)$. Additionally, a total amount of 40 neighbors $(K=40)$ has been set. These two parameters have been fixed according to the analysis carried out in [Fabelo'18a], where it is shown that higher values of both parameters tend to over-smooth the classification result.

$$
d\left(p_{r c}, p_{i j}\right)=\left(P C A_{r c}-P C A_{i j}\right)^{2}+\left((r-i)^{2}+(c-j)^{2}\right)
$$

Considering the neighbor searching implementation onto the MPPA a band window scan has been employed to fit the data into the $\mathrm{CC}$ memory. Specifically, a 14-lines band has been selected and, consequently, 3 regions have been defined. From the upper border of the image till the $7^{\text {th }}$ line (included), the first 14 lines of the image are used (Upper). For the last 7 lines of the image, the equivalent has been done considering the last 14 lines of the image (Lower). Finally, for the middle region of the image, the band window is moved together with the pixel being analyzed (Middle). Figure 7-4 graphically represents this idea.

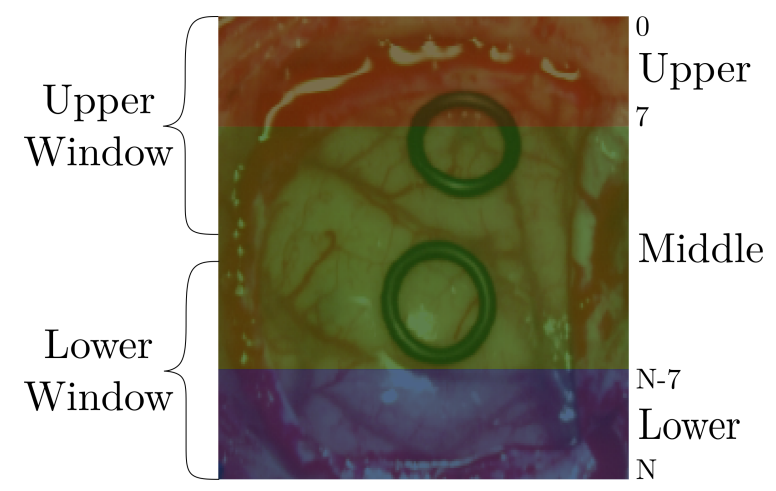

Figure 7-4: Band image regions used during $\mathrm{KNN}$ searching algorithm

From the point of view of the memory footprint, in this algorithm, each pixel is characterized with 3 values (12B): the spectral value associated to the PCA output ( 1 float $-4 \mathrm{~B}$ ) and 2 spatial coordinates, the row and the column, ( 2 int $-8 \mathrm{~B})$. Considering this information and the use of a 14-lines band window during the searching, it has been decided to build blocks of 64 pixels. As a result, most of the data 
will be reused for each block. In total, the size of each image block and its associated window is, in the worst case (dermatological images), $164.81 \mathrm{kB}((1,000 * 14+64) * 12)$.

As a result, the implementation of the algorithm follows Algorithm 7-6. In this algorithm, the input to be used (pixelFeatures) is built in the $\mathrm{IO}$ before starting the execution of the KNN searching. Then, each block of pixels (pixelBlock) composing pixelFeatures is iteratively loaded onto the corresponding CC (line 4). Once the data is stored in the SMEM, for each pixel, the searching starts and 40 neighbors are found for each pixel (line 8). As mentioned before, this distance between pixels is computed using Equation 7-1. Additionally, it should be noted that a brute force searching mode has been employed and that the 40 neighbors are found doing a full analysis of the searching window. Once all the neighbors of the block of pixels are found, they are sent back to the $\mathrm{IO}$ (line 12) so as to begin with the filtering part of the KNN algorithm.

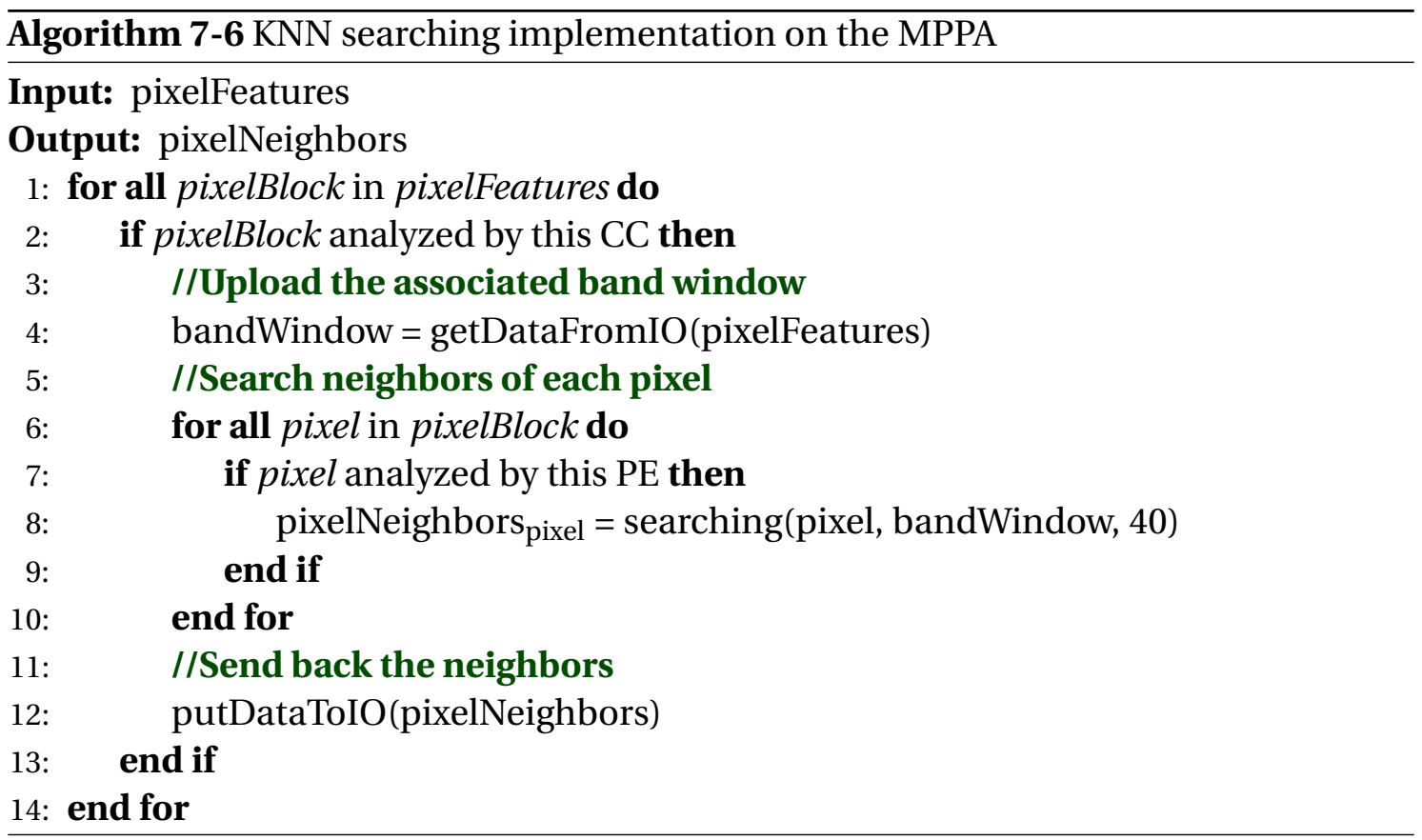

Finally, the last two stages of $\mathrm{KNN}$ algorithm, filtering and class selection, have been combined to optimize the processing. Additionally, as the information required to do the filtering is large compared to the computation itself, these two stages have been implemented on the $\mathrm{IO}$ and are executed right after the $\mathrm{KNN}$ searching has finished. Consequently, as all the information is already stored in the IO, the implementation of these two parts of the algorithm follows Algorithm 7-7. As can be seen, this filtering step consists of averaging the values of the class probabilities obtained with the SVM classification block using the expression shown in Equation 7-2 (from line 4 to 11). After this, the class with the largest probability is selected, as part of the class selection step (line 12). Thanks to the combination of both steps, only one loop going through all the pixels within the hyperspectral image is needed. 


$$
O_{\text {pixel }}=\frac{\sum P_{\text {neighbor }}}{K}, \forall \text { neighbor } \in \text { pixelNeighbors }
$$

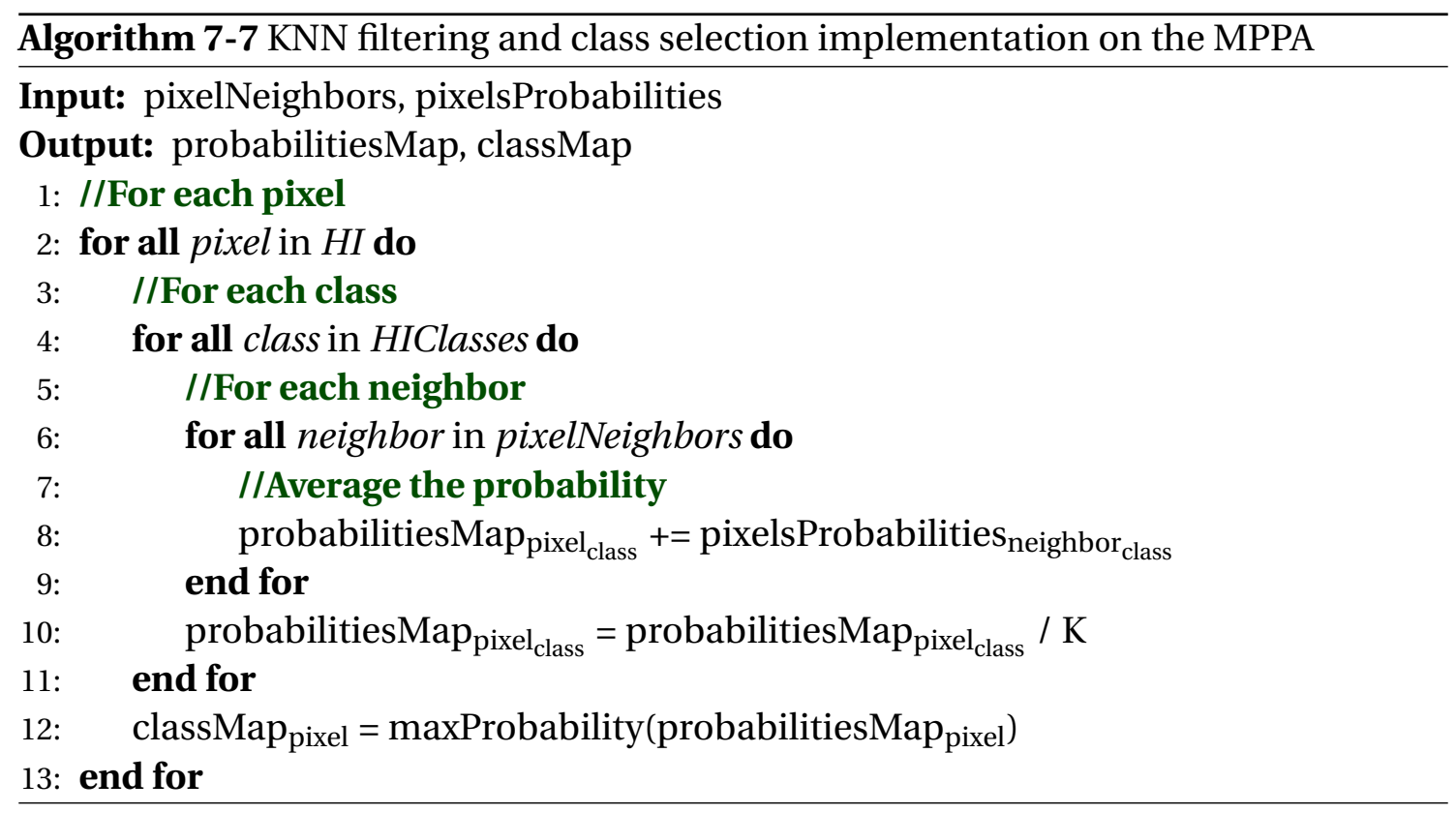

\section{Summary}

Considering the implementations described in this section, as introduced at the beginning of the explanation, the CCs execute the sequence: 1) PCA 2) SVM and 3) KNN searching. Additionally, in parallel to the execution of $\mathrm{PCA}$, the $\mathrm{IO}$ is in charge of doing the transpose of the input matrix and, once PCA finishes, it has to build the featureMatrix that will be used during $\mathrm{KNN}$ searching algorithm. Finally, the $\mathrm{IO}$ is also responsible of the KNN filtering. These steps are not parallelized and, thus, their processing times are independent from the inter- and intra-cluster parallelization strategies. As a result, as shown in Figure 7-5, two situations may happen. On the one hand, as represented in the Ideal case (Figure 7-5a), the IO can execute its tasks faster than the CCs and, thus, the CCs do not enter in idle mode, as they arrive later to the synchronization points. On the other hand, it is possible that the execution of one of these steps on the $\mathrm{IO}$ is slower than the part of the algorithm being executed on the $\mathrm{CC} s$, as represented in Figure 7-5b. In this case, $\mathrm{CC} / \mathrm{s}$ have to go idle and wait for the $\mathrm{IO}$ to finish its processing. 


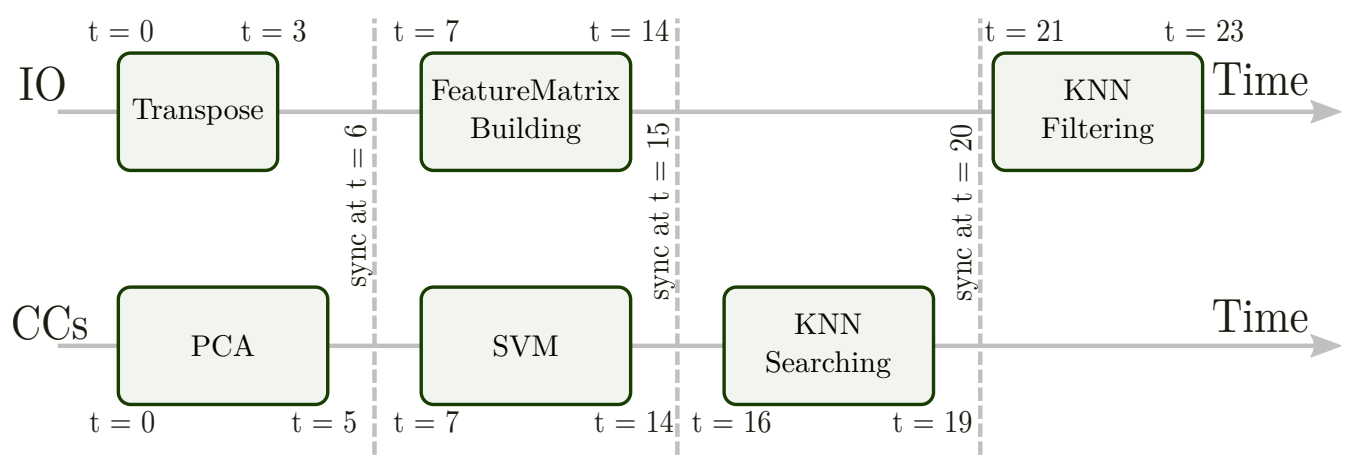

(a) Ideal

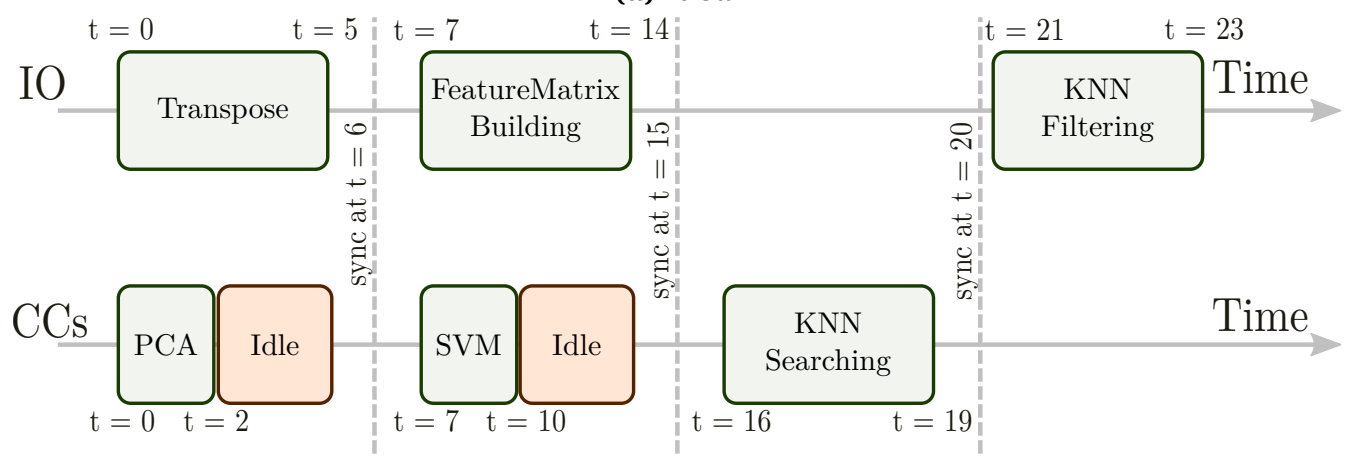

(b) Wait

Figure 7-5: Possible execution workflows of the HSI processing chain implementation done by hand on the MPPA

\subsubsection{Results}

Once the implementation done by hand of the HSI processing chain onto the MPPA has been detailed, the results obtained when analyzing the hyperspectral images presented in Section 7.1 are addressed. First, the processing chain results are presented step by step so as to understand the actual functionality of the application. After this, each part of the implementation is profiled in terms of processing time using the combinations of active resources presented in Section 7.1, which enable interand/or intra-cluster parallelization. Finally, the implementation is characterized in terms of performance (executions per second) and average power consumption (W) as a reference to be used in the following sections.

To illustrate the outcomes of the HSI processing chain and validate its implementation on the MPPA, Table 7-5 compiles the outputs of each algorithm composing it. In this table, first, a Red-Green-Blue (RGB) representation of the hyperspectral image is presented in Image column. Additionally, the 1-band representation of each image, which is obtained thanks to the $\mathrm{PCA}$ algorithm, is collected in $P C A$ column. Finally, as can be seen, in both $S V M$ and $\overline{K N N}$ columns, color maps are used to graphically represent the materials classified in the scene. In this case, healthy tissue is represented in green, tumorous tissue is displayed in red, hyper-vascularized tissue is depicted in blue and background/others are associated to black. 
Table 7-5: HSI processing chain outputs. PCA SVM and KNN results obtained for the MPPA implementation using the validation hyperspectral dataset

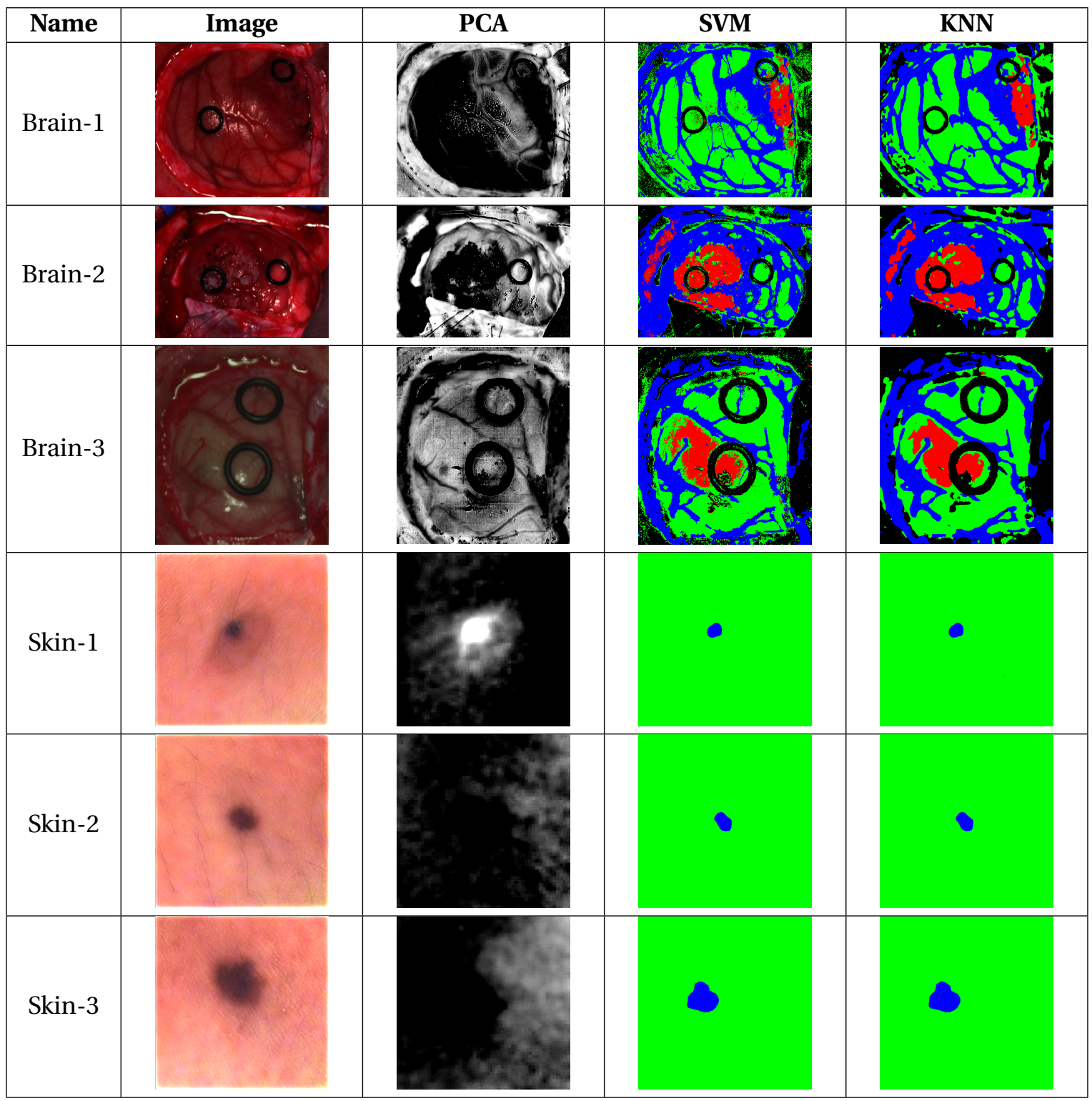

As a summary, Table 7-6 gathers the processing time associated to 1) each algorithm of the processing chain together with the one associated to the whole processing, 2) each MPPA configuration and 3) each image contained in the hyperspectral image dataset from both brain and skin scenarios. In addition, transpose, featureMatrix building and KNN filtering steps are included with the MPPA config being $I O$, as this resource is responsible of executing these steps. Finally, an extra row has been included for each step that is executed on the $\mathrm{CC} s$, containing the total speedup obtained when comparing the sequential execution (Seq) versus the fully-parallel one (MPPA). From these values, several conclusions can be extracted: 
Table 7-6: Partial processing times (s) and speedups of the HSI processing chain. Implementation by hand

\begin{tabular}{|c|c|c|c|c|c|c|c|}
\hline Algorithm & \begin{tabular}{|l|} 
MPPA \\
config
\end{tabular} & Brain-1 & Brain-2 & Brain-3 & Skin-1 & Skin-2 & Skin-3 \\
\hline \multirow{5}{*}{ PCA } & Seq & 34.186 & 28.899 & 19.548 & 96.154 & 96.119 & 96.058 \\
\hline & $1-\mathrm{CC}$ & 4.432 & 3.834 & 2.671 & 12.147 & 12.114 & 12.096 \\
\hline & 1-PE & 2.983 & 2.661 & 1.949 & 7.290 & 7.254 & 7.194 \\
\hline & MPPA & 1.665 & 1.600 & 1.202 & 4.177 & 4.143 & 4.126 \\
\hline & Speedup & 20.530 & 18.066 & 16.262 & 23.021 & 23.198 & 23.280 \\
\hline \multirow{5}{*}{ SVM } & Seq & 26.566 & 20.828 & 14.845 & 55.364 & 55.772 & 55.994 \\
\hline & 1-CC & 1.671 & 1.313 & 0.943 & 3.472 & 3.501 & 3.513 \\
\hline & 1-PE & 1.670 & 1.311 & 0.943 & 3.464 & 3.492 & 3.503 \\
\hline & MPPA & 0.115 & 0.091 & 0.066 & 0.222 & 0.224 & 0.227 \\
\hline & Speedup & 230.047 & 229.240 & 224.718 & 249.129 & 248.728 & 246.619 \\
\hline \multirow{5}{*}{$\begin{array}{c}\text { KNN } \\
\text { searching }\end{array}$} & Seq & 129.596 & 108.795 & 52.939 & $1,090.837$ & $1,090.832$ & $1,090.873$ \\
\hline & 1-CC & 8.378 & 7.045 & 3.433 & 70.262 & 70.261 & 70.264 \\
\hline & 1-PE & 8.122 & 6.840 & 3.331 & 68.228 & 68.229 & 68.230 \\
\hline & MPPA & 0.525 & 0.443 & 0.216 & 4.396 & 4.396 & 4.396 \\
\hline & Speedup & 246.844 & 245.481 & 245.117 & 248.145 & 248.138 & 248.126 \\
\hline Transpose & IO & 0.755 & 0.647 & 0.446 & 2.603 & 2.603 & 2.603 \\
\hline $\begin{array}{c}\text { Feature } \\
\text { Matrix } \\
\text { building }\end{array}$ & IO & 0.005 & 0.005 & 0.003 & 0.024 & 0.024 & 0.024 \\
\hline $\begin{array}{c}\text { KNN } \\
\text { filtering }\end{array}$ & IO & 0.495 & 0.417 & 0.279 & 2.305 & 2.305 & 2.305 \\
\hline
\end{tabular}

- In KNN, where the computation workload is higher than the communication one, the average speedup is $247.01 \times$. This almost ideal speedup is achieved when using the 256 PEs in parallel. In this algorithm, there are almost no synchronizations or idling states and both communications and processing are fully parallelized and managed by the PEs themselves.

- In the case of PCA algorithm, the situation is the opposite, as this algorithm is communication bounded when implemented on the MPPA. The huge amount of transmissions required to process whole bands in both centering and covariance steps restricts the acceleration potential of this algorithm and only an average speedup of $40.56 \times$ is achieved.

- Regarding SVM as in this algorithm the computational part also overcomes the communication, the average speedup obtained is $239.68 \times$. Again, this high speedup is reached thanks to the independence in the communication and computation of each $\mathrm{PE} \mid$ when all of them are simultaneously active.

From these conclusions, it can be deduced that computation-intensive algorithms SVM and KNN are more suitable to be accelerated on the MPPA platform, as 
communication-bounded ones (PCA) are constrained due to the $\mathrm{NoC}$ capabilities. Nevertheless, it should be highlighted that this communication constraint is partially overcome thanks to the use of asynchronous communication protocols. In this sense, as each $\mathrm{PE}$ can manage independently its processing and communication, they can request both read/write data from/to $\mathrm{IO}$ and start the processing as soon as they receive the data readiness notification.

In this line, as shown in Figure 7-5, when measuring the total processing time required to analyze one hyperspectral image, if two stages are executed in parallel on the $\mathrm{IO}$ and on the $\mathrm{CC}$, the one with the highest processing time is the accounted one. The total processing times are graphically represented in Figure 7-6 as a bar plot, following the organization shown in Table 7-6. Specifically, the processing time (in seconds) associated to each MPPA configuration is shown with a different bar color, while each image is displayed as a different tick in the horizontal axis. Additionally, in the figure, the speedup obtained when comparing the processing time of the $M P P A$ configuration with the sequential one for every image is depicted with a blue line.

As can be seen, the total speedup is linked with the spatial resolution of the hyperspectral image being processed, since Brain-3 is the smallest one (49.696×) and Skin ones $(112.129 \times)$ are the biggest in the set of experiments. This difference is mainly associated to the searching step in the KNN algorithm, where a band window is employed. Since this search is done using 14 rows of the image, the time spent in this stage is directly linked to the width of the image. Consequently, the optimization opportunities grow with the size of the image, as clearly represented in Figure 7-5.

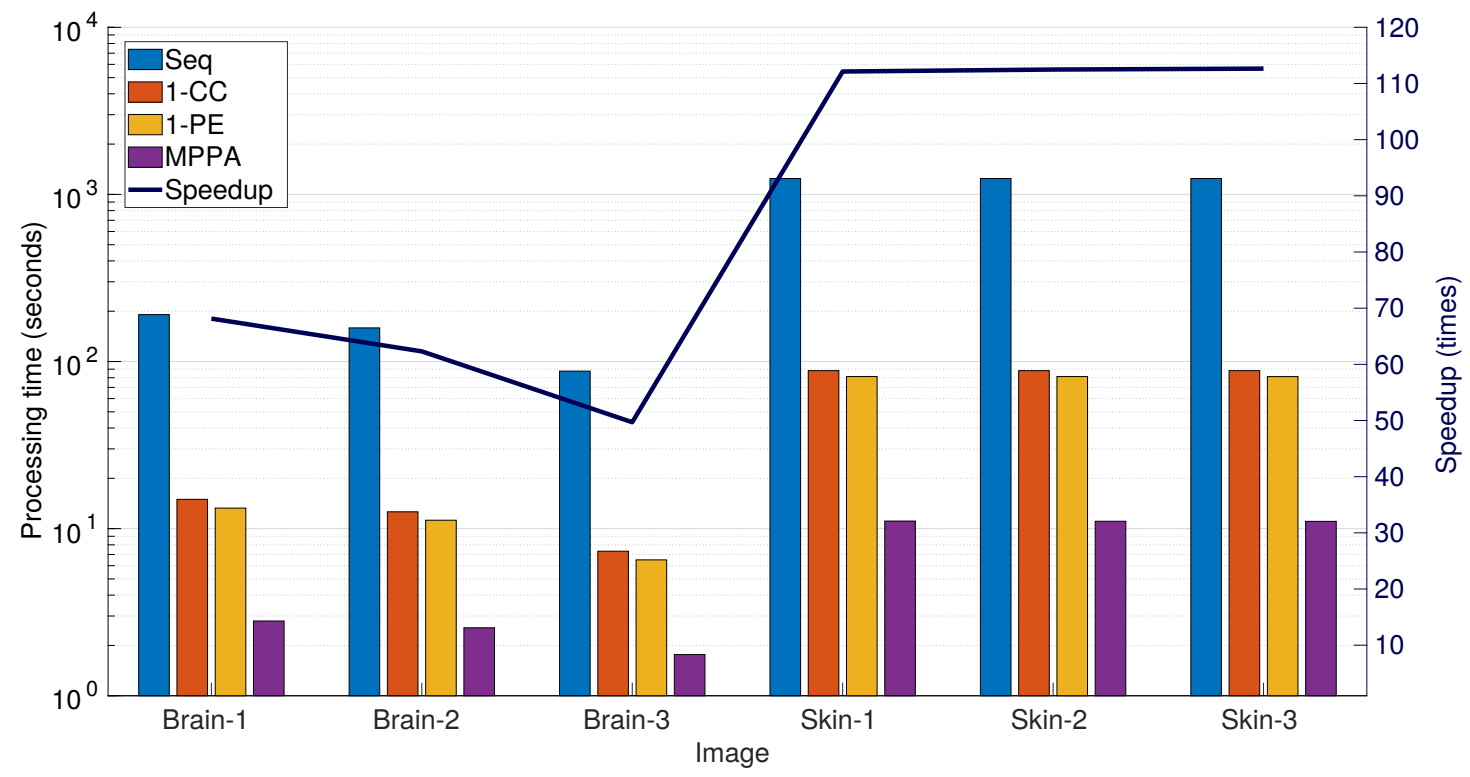

Figure 7-6: Total processing times (s) and speedup (times) of the HSI processing chain. Implementation by hand

Finally, taking into account that this implementation has been developed in the context of HELICoiD project, the main focus has been put on minimizing the 
processing time, i.e, maximizing the performance. As a result, Table 7-7 gathers the characterization of the MPPA configuration for both performance and power consumption. These values will be considered the reference during the validation of the proposed energy-aware design methodology presented in the next sections.

Concerning the performance, the values have been computed following Equation 7-3, where Processing Time has been extracted from Figure 7-6.

$$
\text { Performance }\left[\frac{\text { Images }}{\mathrm{s}}\right]=\frac{1}{\text { ProcessingTime }}
$$

Regarding the power measurement, the procedure is equivalent to the one explained in Section 5.2, i.e. the one used to build the MPPA energy consumption model (Chapter 5). In this case, the processing chain has been iteratively executed during 600 seconds and the average power consumption has been computed.

Table 7-7: Performance (images per second) and power (W) of the HSI processing chain. Implementation by hand

\begin{tabular}{|c|c|c|c|c|c|c|}
\cline { 2 - 7 } \multicolumn{1}{c|}{} & Brain-1 & Brain-2 & Brain-3 & Skin-1 & Skin-2 & Skin-3 \\
\hline $\begin{array}{c}\text { Performance } \\
{[\text { images/s] }}\end{array}$ & 0.357 & 0.392 & 0.567 & 0.090 & 0.090 & 0.090 \\
\hline $\begin{array}{c}\text { Average power } \\
\text { [W] }\end{array}$ & 16.18 & 15.96 & 15.24 & 17.28 & 17.32 & 17.35 \\
\hline
\end{tabular}

\subsection{Design time optimization}

Once the implementation developed by hand for HELICoiD project has been explained and characterized, this section details the implementation of the HSI cancer detection algorithm on the PREESM dataflow design framework. Contrary to the implementation done by hand, in this case both performance and energy consumption are considered when evaluating the application MPPAdeployment. To be precise, first, the constraints considered when implementing the system with a dataflow MoC are explained in Subsection 7.3.1. After this, the actual dataflow implementation of the application is detailed in Subsection 7.3.2, Then, in Subsection 7.3.3, the dataflow version of the HSI processing chain is validated and optimized considering only latency requirements, which means that no energy-awareness is included. This optimization employs PAPIFY to characterize each actor in terms of execution time and PREESM default mapping/scheduling algorithm to generate latency-optimized versions of the system. Finally, energy-awareness is included within the requirements and the associated results are evaluated in Subsection 7.3.4. 


\subsubsection{Dataflow-oriented implementation constraints}

Contrary to the implementation done by hand in Subsection 7.2.1, the implementation presented in this section has to be compliant with the PiSDF MoC, which is the one used in PREESM to describe applications. This model, as introduced in Subsection 3.3.1, defines applications as a set of functional blocks, the so-called actors, connected through communication links, i.e. FIFOs, transmitting data tokens. Specifically, when developing applications in PREESM, it has to be taken into account that actor consumption and production rates are set when designing the application. By doing so, the application parallelism can be specified through the use of parameters. As a result, the same application implementation can be easily configured to work with different scenarios where, for example, the image resolution varies and/or a different parallelization degree is required.

However, considering that the use-case is an HSI processing chain working on the MPPA, 3 constraints need to be taken into account: 1) the parallelism degree, 2) the workload distribution and 3) the communication-processing strategy.

First, considering the parallelism degree that can be used to specify the application, it should be considered that, as PREESM bases its application generation procedure in graph management, huge parallelism degrees may lead to unmanageable graph specifications. As stated in Subsection 3.3.1, one of the first steps of this procedure is to flatten the initial PiSDF graph in a SRDAG, where the parallelization of the application is explicitly exposed. Consequently, each actor is replicated as many times as firings will happen in a single iteration of the whole application graph, as represented in Subsection 3.3.1. After this, the rest of PREESM workflow works with this flattened representation of the dataflow application. On top of that, as highlighted in [Hascoet'18, Brucker'07], it should be considered that mapping and scheduling time exponentially increases when the number of both actors and $\mathrm{PE} s$ increases.

In this regard, in the MPPA dataflow implementation of the HSI processing chain, a level of parallelism equivalent to the one presented in Subsection 7.3.2 would need more than 20,000 actors. Due to the small amount of memory available in each CC, the covariance matrix computation has to be performed using vector multiplications, implying a parallelism equal to band $*$ bands, e.g., 16,384 actors for Brain images. As mentioned before, the time required by PREESM to map and schedule this huge number of actors would be unmanageable.

To tackle this problematic, the concept of tensors [Zhang'12] is applied. As exemplified in Figure 7-7, these tensors are used to represent the hyperspectral images matching dimensions with the so-called modes. In Figure 7-7a, the image is considered a third-order tensor, as it has 3 modes: height, width and bands. In order to be able to store more than one band in each CC, Figure 7-7b represents the same image, but divided into several tensors where the height mode is, as an example, divided by 4 . With this approach, processing a complete hyperspectral image would require as many iterations as tensors are created. Nevertheless, in the dataflow implementation, the parallelism required to deploy the application on the MPPA can 
be modulated using more or less tensors. As an example, if using 4 tensors in the dataflow implementation implies that 4 hyperspectral bands fit in the CC memory, the covariance matrix computation parallelism could be reduced by 4, i.e., 4,096 actors for Brain images, hence reducing the number of actors PREESM has to map and schedule.

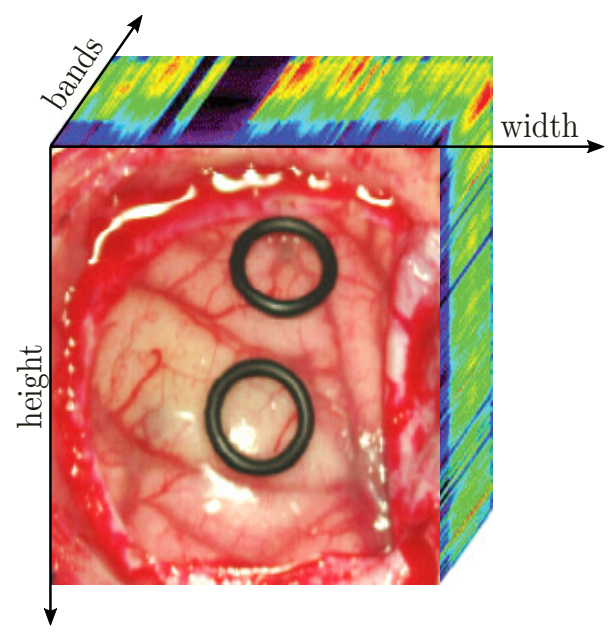

(a) Original hyperspectral image

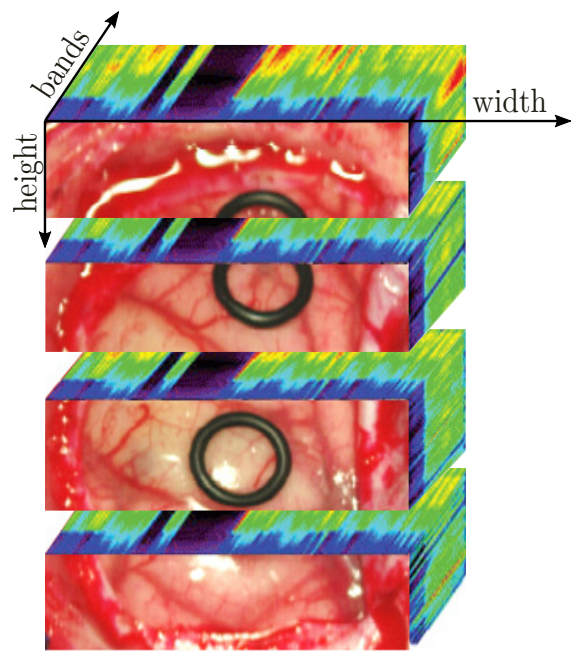

(b) 4-tensor representation

Figure 7-7: Third-order tensor representation of hyperspectral images: (a) original and (b) 4tensor representation

Secondly, due to the nature of PREESM and PiSDF itself, the workload distribution must be uniform. That is, when the workload is distributed among several parallel instances of the same actor, although each instance can work with different data, it must consume and produce the same number of tokens. Additionally, information entering one actor cannot be shared nor reused by other actors. As a consequence, optimizations like the one performed for the covariance in the implementation done by hand Algorithm 7-2 - cannot be applied. In that optimization, 1) band blocks were shared among the $\mathrm{PE}$ s of one $\mathrm{CC}$ and 2) as only the upper triangle of the resulting matrix was computed, the workload differed for each row of the covariance matrix. On the contrary, in the dataflow implementation, the whole covariance matrix must be computed and, consequently, a performance loss is expected.

Finally, in the specific case of PREESM MPPA backend, the communicationprocessing strategy differs from the one employed in Subsection 7.2.1. Although PREESM allows clustering actors to distribute the workload among the PEs of one CC. they cannot share any data, as explained in the previous constraint. In this regard, and taking into account the memory limitations of the MPPA it has been decided to perform intra-cluster parallelization by means of OpenMP clauses. As a consequence, as graphically represented in Figure 7-8, the dataflow implementation will use only 16 communication channels when communicating both $\mathrm{IO}$ and $\mathrm{CC}$ s. Then, each CC will parallelize the processing among its $16 \mathrm{PE}$. This new communication-processing strategy will also imply a performance detriment in the dataflow implementation. 


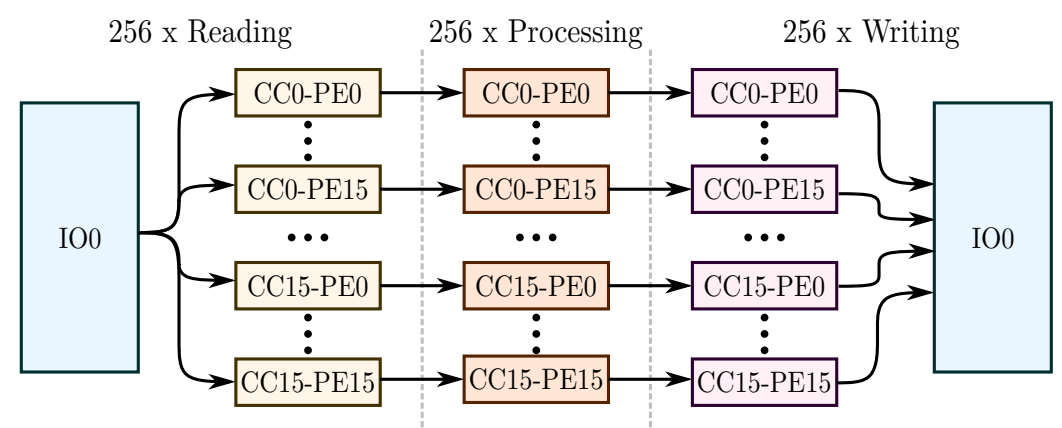

(a) Implementation by hand $16 \times$ Reading $\quad 16 \times 16 \times$ Processing $\quad 16 \times$ Writing

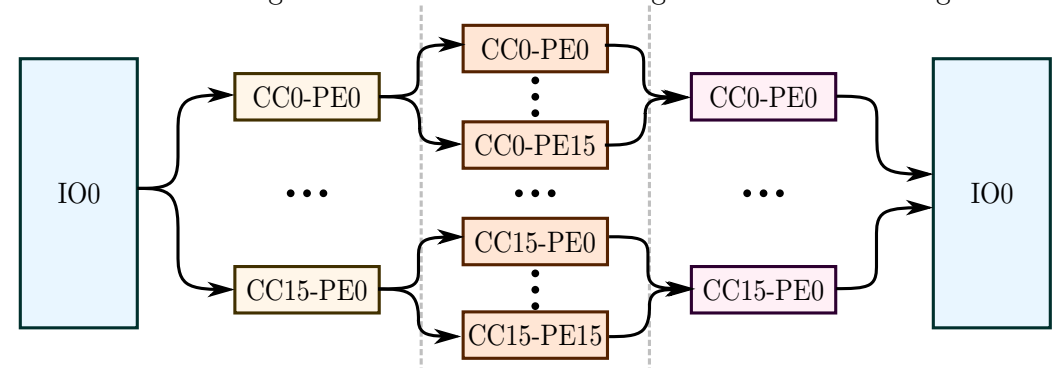

(b) Dataflow implementation

Figure 7-8: MPPA communication-processing strategies comparison: (a) implementation by hand and (b) dataflow implementation

\subsubsection{Dataflow implementation}

Considering the aforementioned constraints, the PREESM version of the HSI cancer detection application, from an architecture-independent point of view, is detailed in this subsection. It should be noted that, during the whole application development, both hierarchies and parameters have been employed. On the hierarchy side, the design of the dataflow application has been built as a set of modules or blocks, where each part of the algorithm is independently implemented. Additionally, parameters have been used to express both input application parameters, e.g., spatial and spectral resolutions, and the parallelism of the application, as will be detailed further in this section. In Subsection 3.3.1 both PREESM and PiSDF are explained in detail.

To be precise, this subsection presents the different networks composing the HSI cancer detection application using a top-down approach. First, the systemlevel networks, which define the application with a high level of abstraction, are introduced. Then, the networks linked to PCA SVM and KNN are outlined. Lastly, some final remarks including a summary of the different parameters used to configure the application graph are highlighted. 


\section{System-level}

First, the highest hierarchy level is shown in Figure 7-9, In this figure, the system is represented as a set of 3 actors (Read_HS_Image, HSI_cancer_detection and Write_results) and the input application parameters. Specifically, in this level the parameters that are defined are: 1) the spatial resolution (columns and rows); 2) the spectral resolution (bands); 3) the number of classes, or elements, that will be identified within the hyperspectral scene (numClasses); and 4) the number of tensors in which the input image is divided (numTensors). As a consequence, in this implementation, the graph needs to be executed numTensors times so as to process the whole hyperspectral image.

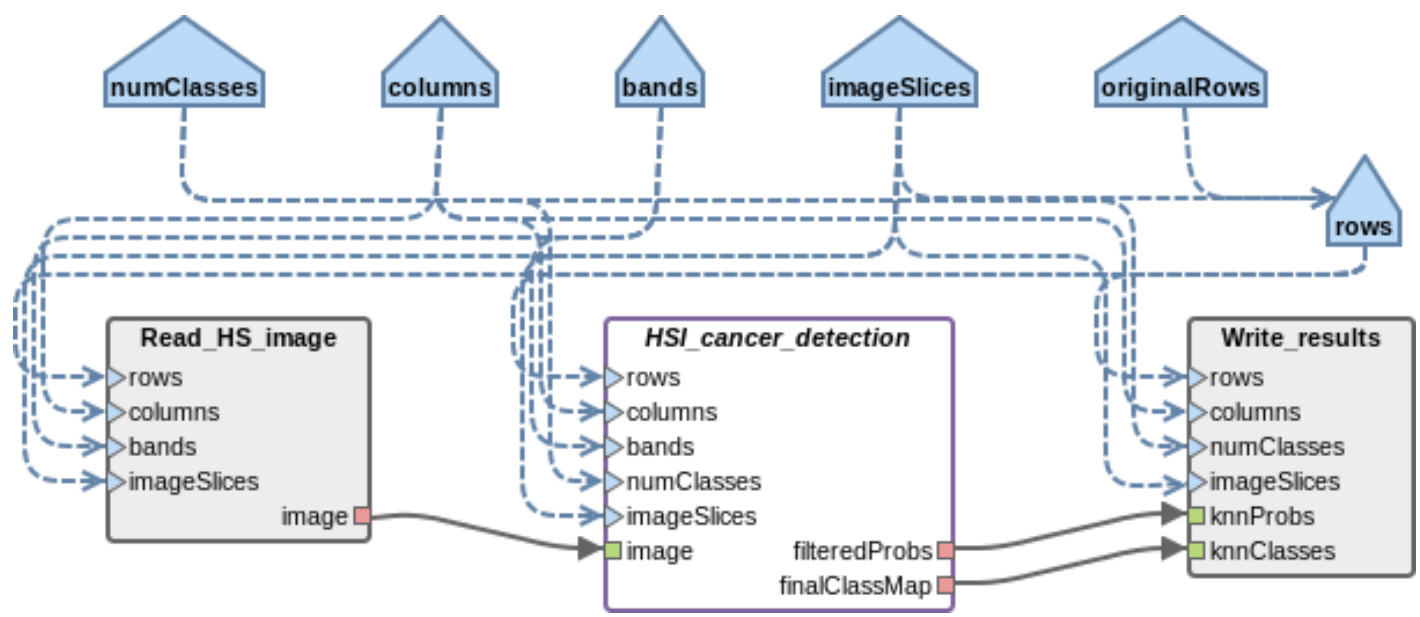

Figure 7-9: HSI processing chain in PREESM system-level representation

In the second level of the dataflow graph hierarchy, represented in Figure 7-10, the algorithms composing the HSI processing chain are shown. From this graph, analyzing the dependencies among blocks, it can be observed that both DimensionalityReduction (PCA algorithm) and SupervisedClassification (SVM algorithm) can be executed in parallel. Additionally, SVM requires a transposition of the input image before being executed, performed by the TransposeImage actor. Once these actors finish, the third block of the processing chain, the SpatialSpectralFiltering (KNN algorithm), can be executed. Thanks to this hierarchical implementation, the details of each algorithm are isolated from each other, leading to an easier validation of the HSI processing chain. Finally, it is worth pointing out the use of a broadcast actor (represented in brown). In this work, broadcast actors have been employed to re-use memory pointers and, thus, avoid unnecessary copies of memory in read-only input variables.

Finally, for each algorithm composing the HSI processing chain, i.e., PCA, SVM and KNN, an independent network has been defined. In each case, the corresponding algorithm has been divided in a set of data-independent blocks, which have been associated to actors. Additionally, the intrinsic parallelism corresponding to each stage is controlled via PiSDF parameters. By doing so, the level of parallelism used for each algorithm actor is independently configured. 


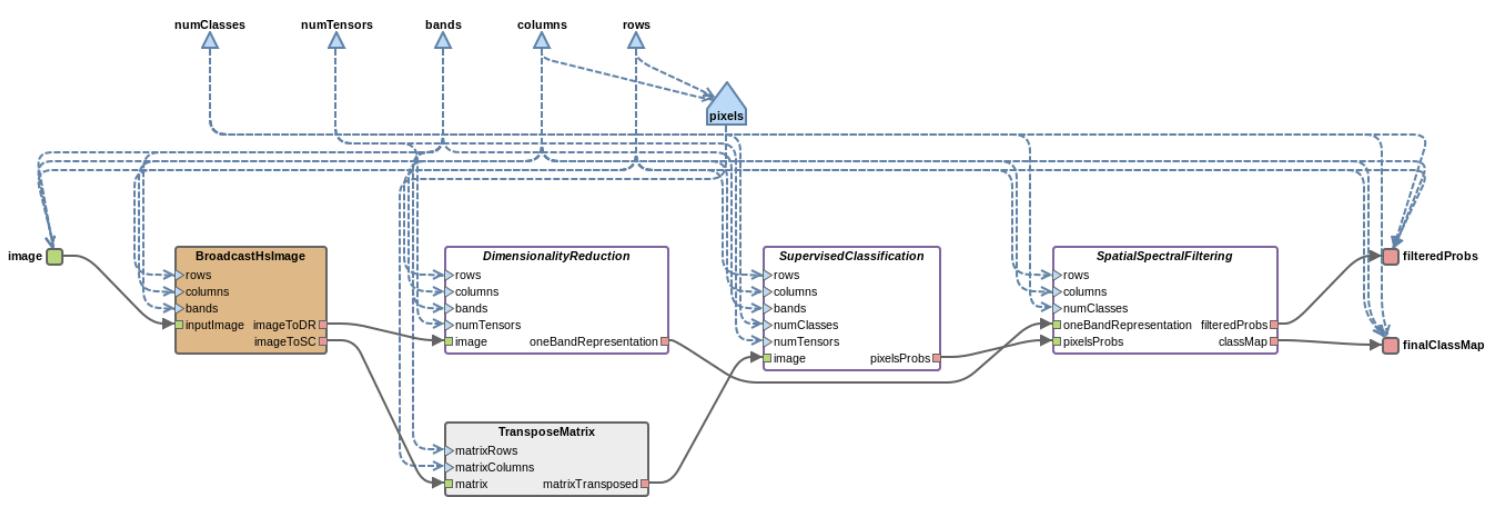

Figure 7-10: HSI processing chain in PREESM processing chain representation

\section{PCA}

In the case of $\mathrm{PCA}$ algorithm, the dataflow graph representation is shown in Figure 7-11. As can be seen, the dataflow implementation is composed of the 4 steps explained in Subsection 7.2.1, which are ImageCentering, Covariance, Jacobi and Projection actors. In the case of the ImageCentering actor, in turn, it is composed of the dataflow network depicted in Figure 7-12, where the two stages composing this step are isolated from each other.

Additionally, during the implementation of this algorithm, both parameters and broadcast actors are combined to express parallelism. For PCA the level of parallelism can be independently modified in each algorithm step:

- ImageCentering: parallelismImageCentering parameter is used to set the number of bands that will process each actor parallel instance.

- Covariance: parallelismCovarianceMatrixA and parallelismCovarianceMatrixB parameters define the parallelism of this stage. The former specifies the number of rows of the resulting matrix, the so-called covariance matrix, that are computed by each actor parallel instance. The latter, under the constraint of parallelismCovarianceMatrixA being equal to bands, defines the number of columns of one row that each actor parallel instance calculates. This constraint exists due to the ordering of the result of each parallel instance, as they are considered blocks and, thus, only values of the same row can be properly placed. Additionally, as introduced in Subsection 7.3.1, all the values of the covariance matrix are computed and no extra optimization is performed. Hence, the 3 broadcast actors are in charge of splitting and distributing the input matrix rows among the parallel covariance actor instances.

- Jacobi: no parallelism is used in this stage.

- Projection: parallelismProjection parameter is used to set the number of pixels that will process each actor parallel instance. In this case, the eigenvector is broadcasted to every actor instance, as actors do not share information. 


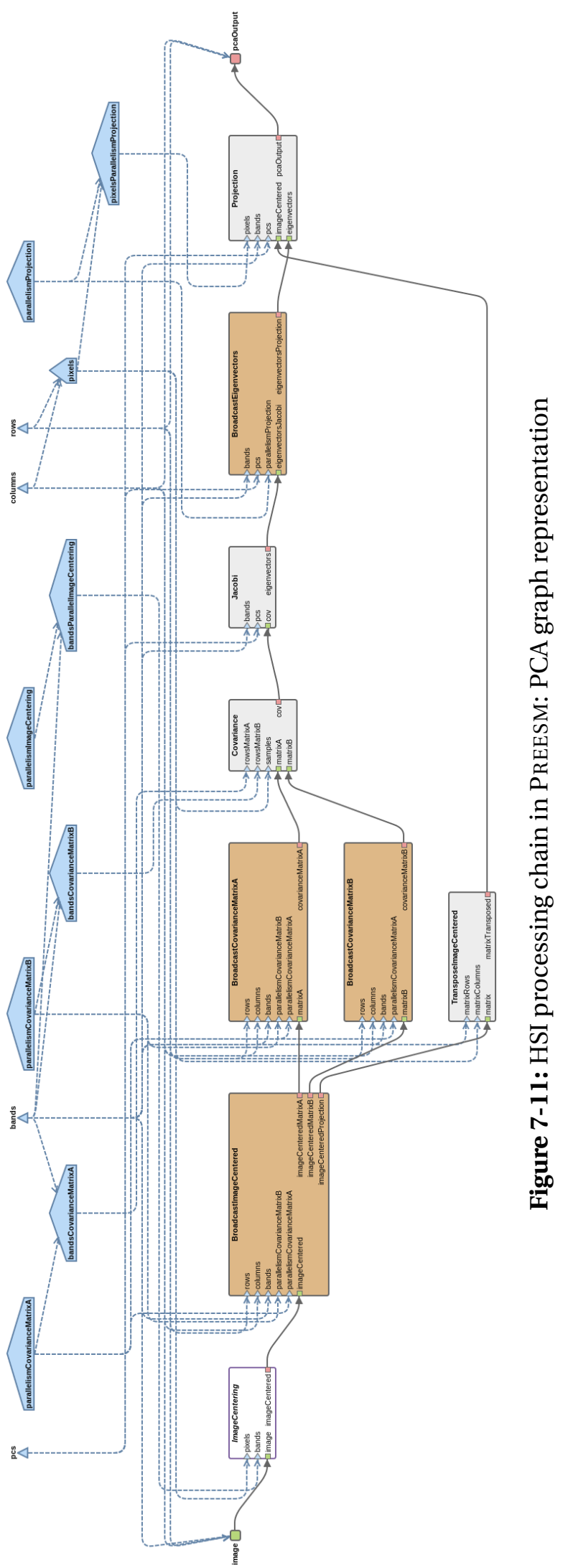




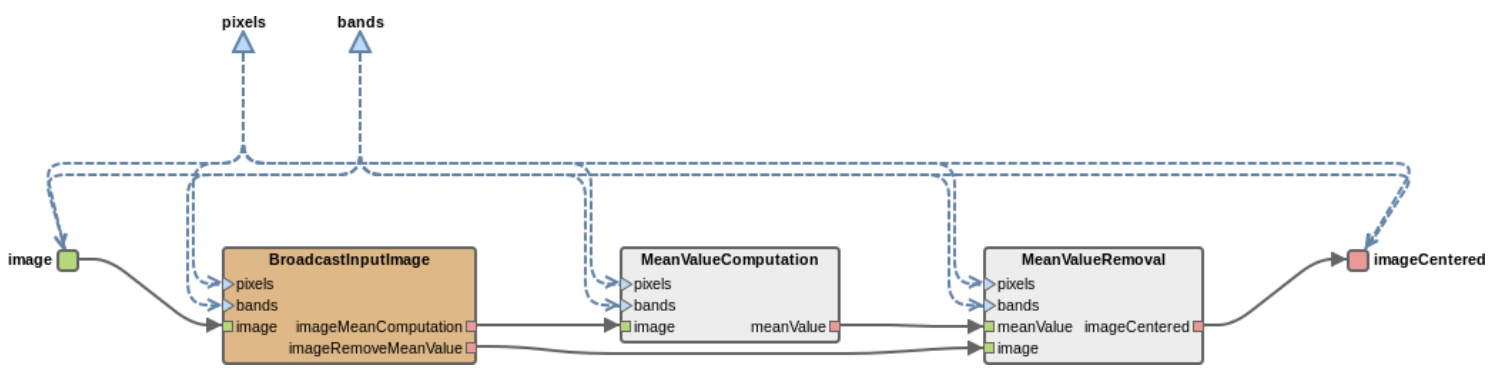

Figure 7-12: HSI processing chain in PREESM image centering graph representation

\section{SVM}

Regarding SVM algorithm, it has been defined using two networks: Figure 7-13 and Figure 7-14. In Figure 7-13, the classification model, which has been previously generated following the methodology explained in [Madroñal'17b], is read and the parallelism of SVM actor is defined through parallelismSVM parameter. Specifically, this parameter defines the number of actors that contains the network shown in Figure 7-14 that will be instantiated when generating the code. As a result, in each SVM actor instance, only a subset of pixels will be classified.

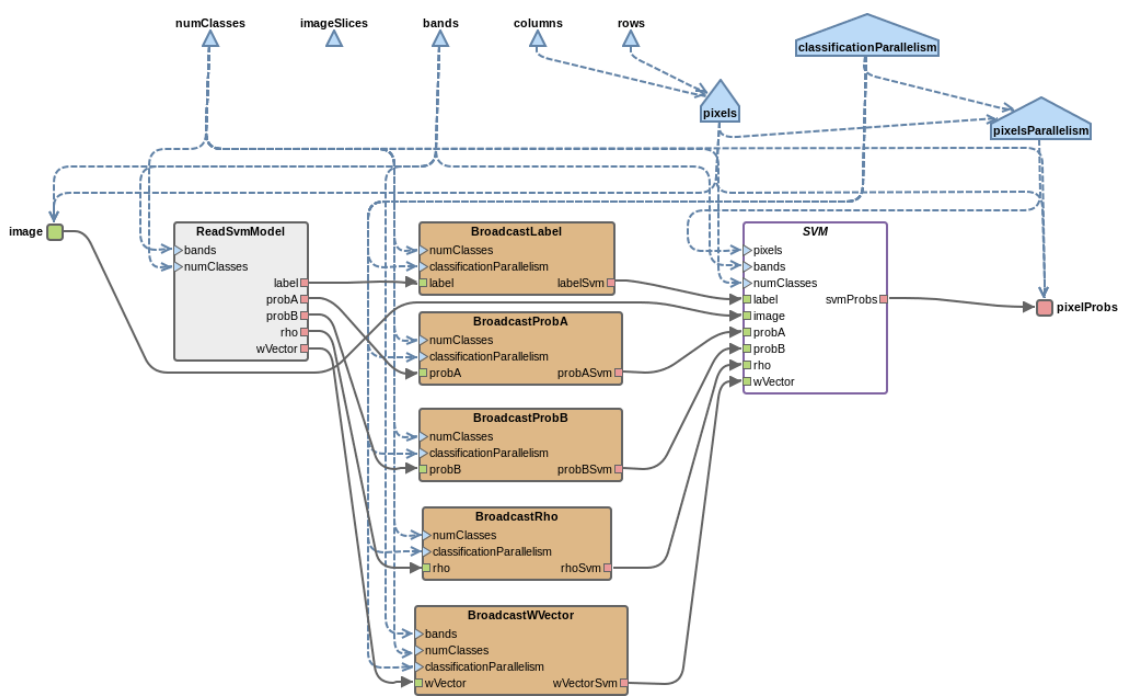

Figure 7-13: HSI processing chain in PREESM SVM top graph representation 


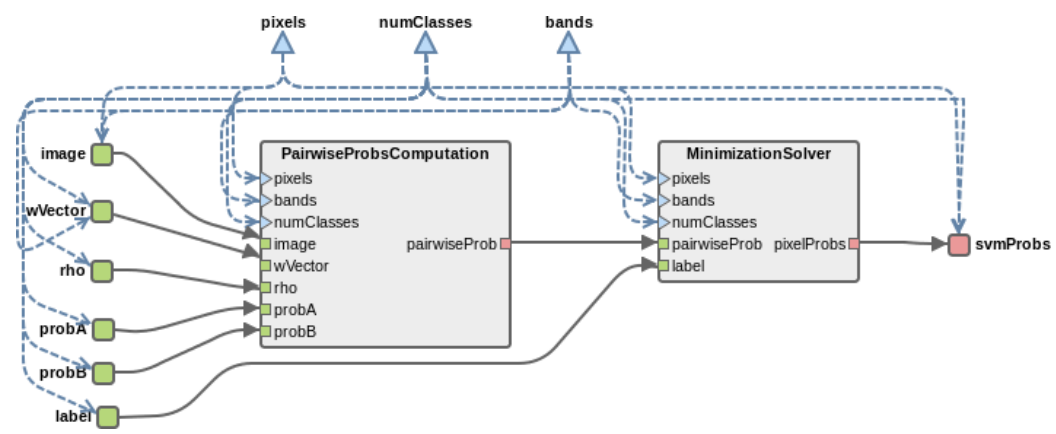

Figure 7-14: HSI processing chain in PREESM SVM stages graph representation

\section{KNN}

Finally, the KNN graph specification is shown in Figure 7-15. In this network, all the parameters configuring the KNN-based filtering are defined: windowSizeKNN, neighbors and lambda. These parameters take the values explained in Subsection 7.2.1. Additionally, parallelismKNN parameter has been included to control the number of horizontal slices in which the KNN-based filtering is parallelized.

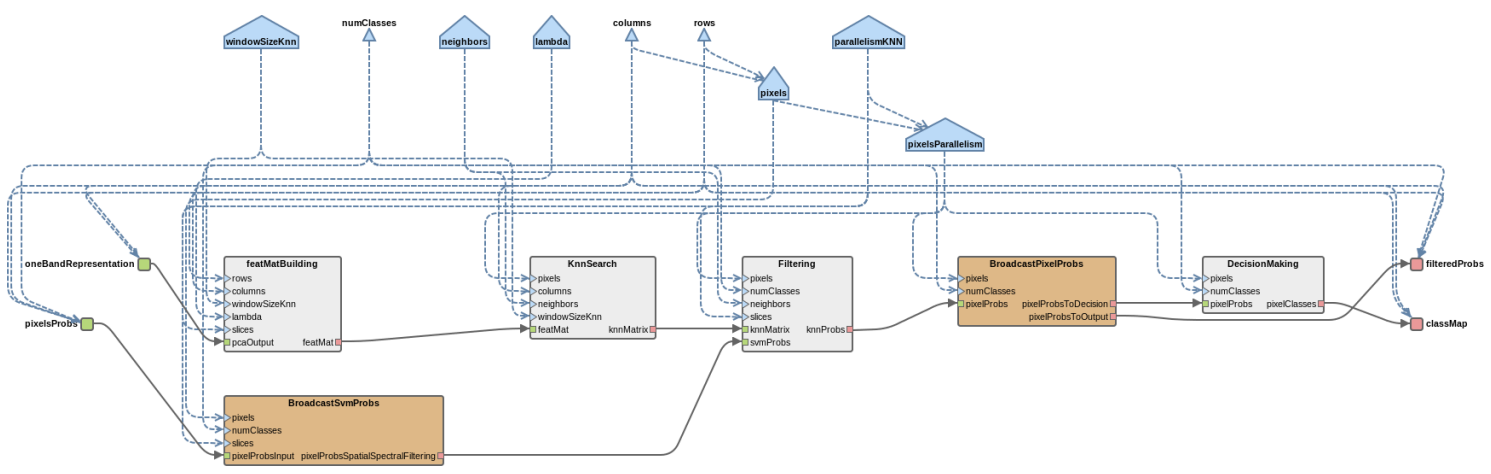

Figure 7-15: HSI processing chain in PREESM KNN graph representation

\section{Final remarks}

Although this application specification has been developed taking into consideration the memory limitations of the MPPA architecture, it can be used to generate implementations no matter the architecture that is targeted. For example, in architectures where the memory is not a constraint, numTensors can be set to 1 and, as a result, the whole image will be processed in only one iteration. Additionally, it should be considered that, since the whole application has been parameterized, it can be easily adapted to different input data and/or target architecture parallelism. As a summary, all the parameters used to configure the network are gathered in Table 7-8. 
Table 7-8: Parameters used in the dataflow implementation of the HSI processing chain

General configuration

\begin{tabular}{|c|c|}
\hline Parameter name & Brief description \\
\hline rows & Height of the input image \\
\hline columns & Width of the input image \\
\hline bands & Spectral bands of the input image \\
\hline numClasses & Number of elements to be identified in the image \\
\hline numTensors & Number of tensors in which the input image is divided \\
\hline
\end{tabular}

PCA

\begin{tabular}{|c|c|}
\hline Parameter name & Brief description \\
\hline parallelismImageCentering & Band blocks used to process the image centering step \\
\hline parallelismCovarianceMatrixA & $\begin{array}{c}\text { First level of parallelism in covariance computation } \\
\text { Rows processed in parallel }\end{array}$ \\
\hline parallelismCovarianceMatrixB & $\begin{array}{c}\text { Second level of parallelism in covariance computation } \\
\text { Columns of each row processed in parallel }\end{array}$ \\
\hline parallelismProjection & Pixel blocks used to process the projection step \\
\hline
\end{tabular}

SVM

\begin{tabular}{|c|c|}
\hline Parameter name & Brief description \\
\hline parallelismSVM & Pixel blocks used in this algorithm \\
\hline
\end{tabular}

KNN

\begin{tabular}{|c|c|}
\hline Parameter name & Brief description \\
\hline windowSizeKNN & Rows of the searching band window \\
\hline neighbors & Number of non-local neighbors to be found \\
\hline lambda & Weight between spatial and spectral contributions \\
\hline parallelismKNN & Pixel blocks used in this algorithm \\
\hline
\end{tabular}

\subsubsection{Latency-based application optimization}

This section presents the results associated to two studies carried out using the PREESM version of the HSI cancer detection processing chain, namely accuracy characterization and latency-based optimization.

On the one hand, the dataflow implementation, which includes the use of several tensors as a novelty, is characterized in terms of accuracy. In this implementation some errors may have introduced, since, for example, in the KNN algorithm, the band window used to search the neighbors of one pixel, represented in Figure 7-4, may change when processing the image using several independent tensors.

On the other hand, the design time optimization loop presented in Section 6.3 is used to iteratively optimize the system in terms of latency without considering energy constraints. To do so, PAPIFY retrieves timing information from the application generated by PREESM and, then, this information is used to update PREESM scenario timing data. With this refined data, PREESM enhances its mapping/scheduling decisions in the following iterations of the application generation mechanism. 
Prior to carrying out the validation of this implementation, the values of PREESM parameters used to specialize each hyperspectral image implementation are defined. These values are gathered in Table 7-9, where 4 sections can be identified:

- Inputs: for each image within the validation dataset, the input parameters (columns, rows and bands) are set. Additionally, as the algorithm configuration remains independent from the input image, these values are equivalent to the ones used in Subsection 7.2.1 for the whole dataset. For example, columns, rows and bands for Brain-1 are 496, 442 and 128, respectively.

- Tensors: as introduced before, to tackle the massive parallelism required to efficiently use the MPPA while maintaining under reasonable limits the number of generated actors, the use of tensors has been included within the dataflow specification. To determine the number of tensors (numTensors) used for each image, the tensor size has been limited to $7 \mathrm{MB}$, which provides a good trade-off between the level of parallelism and the data managed by each CC. For example, Brain-1 is processed using 17 independent tensors, composed of 26 rows each.

- Parallelization: considering the actual size of each tensor (columns and rowsTensor), the parallelization degree of each part of the processing chain is set. These values are included in Table 7-9 with the pattern parallelismSTEPNAME, with STEPNAME being substituted by the corresponding part of the processing chain. Specifically, band/pixel blocks whose total size is up to $1 \mathrm{MB}$ are built. In these cells, two values are provided: the number of parallel instances PREESM will instantiate and, in brackets, the number of bands/pixels that will be processed by each parallel instance. For example, in Brain-1, 16 parallel instances of Centering actors will be instantiated, where each of them will process 8 bands.

- Implementation: based on the specialization done for each image, some interesting figures are added to the aforementioned table: 1) the total number of actors mapped and scheduled by PREESM (Actors), 2) their associated FIFOs (FIFOs) and 3) the memory reserved for data in each CC during code generation (MemorySize). For example, Brain-1 will require 1,465 Actors, 3,850 FIFOs and $877.06 \mathrm{kB}$ of memory in each active CC.

After defining the number of tensors in which the processing of each hyperspectral image is divided, its impact in the accuracy of the dataflow implementation can be characterized. To compute this accuracy loss, the final classification maps, i.e., the KNN output, obtained with the dataflow implementation are compared with the counterpart results generated by the implementation done by hand, which are shown in Subsection 7.2.2. Specifically, one pixel will be considered wrongly classified if it is associated to a different class in each implementation; hence, the total accuracy loss will be computed as the total mislabeled pixels divided by the total number of pixels composing the hyperspectral image. 


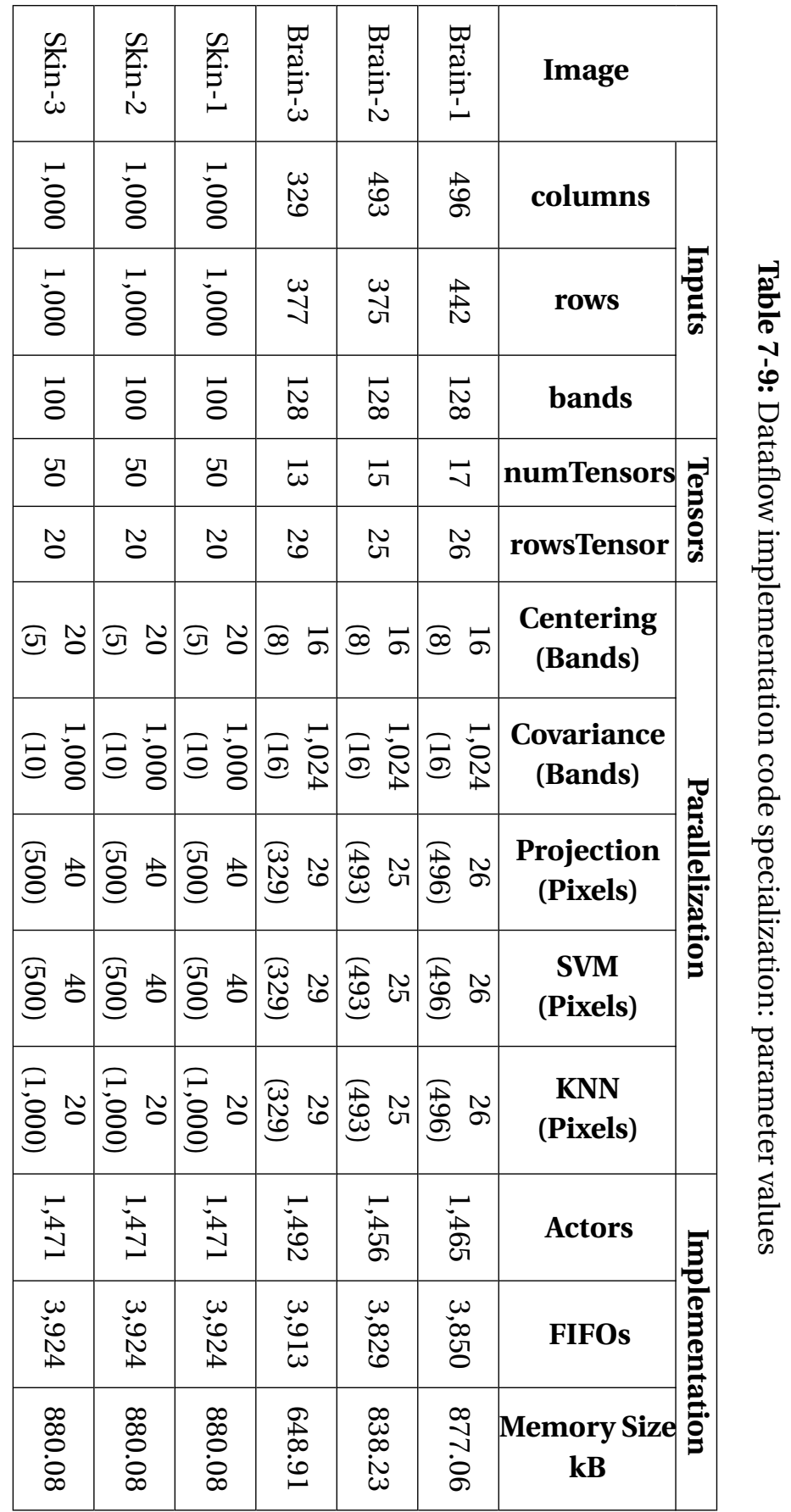


As a result, the maximum accuracy loss is obtained when classifying Brain-3 image, reaching a $2.584 \%$ of mislabeled pixels $(3,205$ pixels out of 124,033$)$. To graphically analyze the effect of using several tensors to process this image, Figure 7-16 presents the final classification maps for the implementation done by hand, Figure 7-16a, and the dataflow one, Figure 7-16b. In this comparison, it can be concluded that the differences are almost negligible and associated to the borders that appear due to the use of isolated tensors. Since each tensor is independently processed, the pixels composing the band window used during the KNN searching, represented in Figure 7-4, differ from the ones used when the hyperspectral image is processed using only one tensor. Therefore, the non-local neighbors of one pixel may differ and, thus, the class associated by the KNNblock may vary too. As an example, in Figure 7-16, one of the tensor borders is surrounded with white dotted lines.

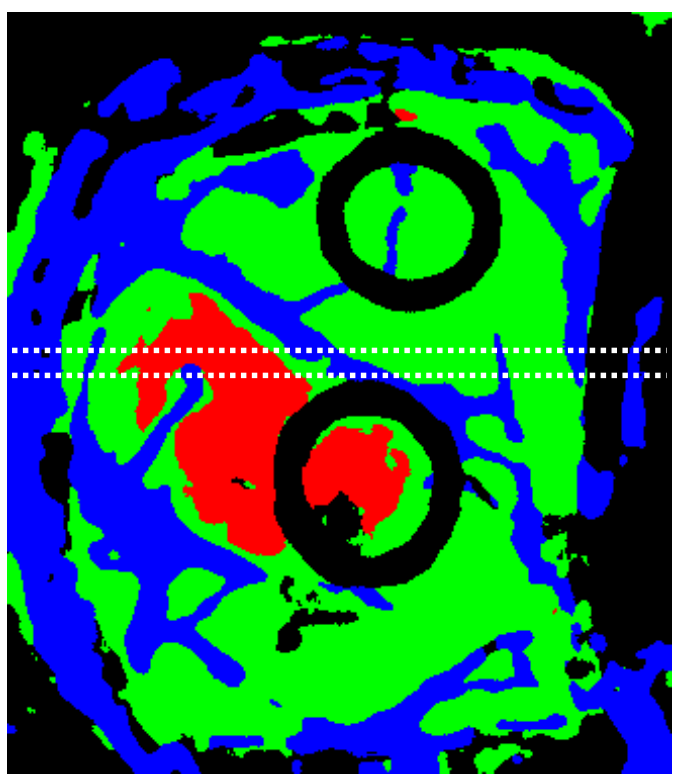

(a) Implementation done by hand

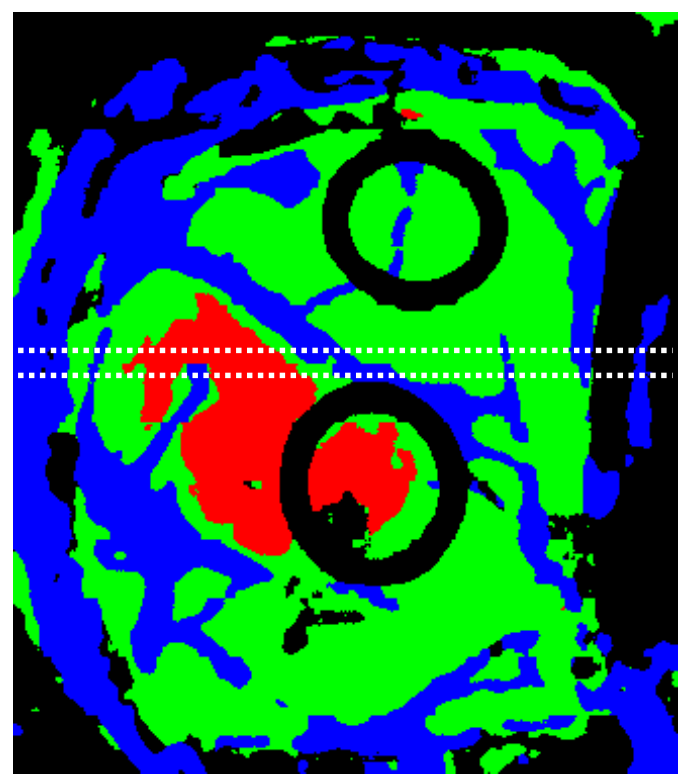

(b) Dataflow implementation

Figure 7-16: Classification results comparison: a) implementation done by hand vs b) dataflow implementation. The white dotted lines surround a border that appears with the use of tensors

Once the dataflow implementation results have been proven correct, the optimization loop presented in Section 6.3 will be used to optimize this implementation in terms of latency. As a reminder, this optimization is based on an iterative procedure where PREESM generates systems using a latency-based mapping/scheduling algorithm based on the information that is available on its scenario file. Then, PAPIFY is used to characterize the deployed application in terms of execution time and the scenario timing information is updated accordingly. Once the scenario data has been refined, PREESM can enhance the mapping and scheduling of the application. Hereafter, each iteration of the optimization loop will be referred as LoopIter.

The results extracted while optimizing latency are gathered in Table 7-10. In this study, the different code generations are tagged following the pattern LoopIter-\#, 
where, as mentioned before, \# represents the number of iterations of the optimization loop already done. Additionally, for each LoopIter-\#, two values are shown: Actors \% and Perf. The former, Actors \%, represents the percentage of actors that have been profiled in previous loop iterations and, consequently, that have been updated in PREESM scenario. To be precise, being the MPPA the target architecture, half of the percentage is linked to actors being executed on the $\mathrm{IO}$ and the other half represents the ones being executed on the CCs. The latter, Perf, shows the performance achieved, represented as complete hyperspectral images processed per second, independently of the number of tensors (iterations) used to process it.

It is important to take into account that, for each LoopIter-\#, two executions are carried out. First, PAPIFY is disabled so as to take the values represented in Perf columns. Secondly, PAPIFY instrumentation is enabled to characterize the specific execution times that are linked to each actor-PEpair. This instrumentation involves an overhead that reaches, in the worst case, a 20\%. Nevertheless, as the profiling targets the Timing information refinement in PREESM scenario, no overhead is found in the final prototype, as PAPIFY is disabled.

As can be seen, after profiling 3 generated codes (LoopIter-3), all the actors are characterized for every hyperspectral image. Hence, the performance value shown in its associated Perf column is the maximum one that can be achieved using PREESM. Comparing these results with the ones obtained in Subsection 7.2.2, there is a clear performance loss. In general, the performances achieved with the dataflow implementation are around 6 times lower than the implementation done by hand. The rationale behind this performance detriment is aligned with the ideas exposed in Subsection 7.3.1, where this performance loss had already been foreseen.

Focusing the analysis on the values obtained for each loop iteration of the latencybased optimization procedure, some interesting details can be observed:

- LoopIter-0: in this case, the time associated to every actor running on every type of $\mathrm{PE}$ is considered the same. Consequently, during the mapping/scheduling process, these actors are mapped onto the $\mathrm{PE}$ by only taking into consideration their dependencies, i.e., the scheduling order, and the associated data transmissions. To be precise, in this case, all the actors have the default timing value set by PREESM, i.e., 100 units of time. It should be noted that, as PAPIFY provides timing information in $\mu$ s, the employed unit of time is, thus, $\mu \mathrm{s}$.

- LoopIter-1: after one application profiling, a general performance detriment is observed. In this iteration of the process, up to an $80 \%$ of the timings have been added to the scenario file but, from PREESM point of view, the other $20 \%$ require $100 \mu \mathrm{s}$ to be executed. Specifically, Jacobi actor, which is one with the largest execution times, has been executed on a CC in LoopIter- 0 taking, at least, 100ms. As a result, in LoopIter-1, PREESM decides to map Jacobi actor on the IO, which results in a slower execution. This is the reason why a performance detriment is observed in this iteration of the process. 
- LoopIter-2: at this point, almost the whole set of actors has been profiled in every implementation, so the performances obtained overcome the ones from the first iteration. Specifically, the implementation optimized for Brain-3 already has the whole actor set characterized, hence reaching its highest performance.

- LoopIter-3: in this last iteration of the optimization loop, all the actors have been characterized. As a result, all the implementations reach the best performances that can be achieved with the current PREESM dataflow implementation.

Table 7-10: PREESM latency optimization using PAPIFY timing profiling. Performance is provided in hyperspectral images processed per second and actors updated in \%

\begin{tabular}{|c|c|c|c|c|c|c|c|c|}
\hline \multirow{2}{*}{ Image } & \multicolumn{2}{|c|}{ LoopIter-0 } & \multicolumn{2}{c|}{ LoopIter-1 } & \multicolumn{2}{c|}{ LoopIter-2 } & \multicolumn{2}{c|}{ LoopIter-3 } \\
\cline { 2 - 9 } & Actors \% & Perf & Actors \% & Perf & Actors \% & Perf & Actors \% & Perf \\
\hline Brain-1 & 0 & 0.076 & 75.86 & 0.047 & 93.10 & 0.081 & 100 & 0.083 \\
\hline Brain-2 & 0 & 0.093 & 75.86 & 0.057 & 93.10 & 0.099 & 100 & 0.102 \\
\hline Brain-3 & 0 & 0.139 & 79.31 & 0.082 & 100 & 0.150 & - & - \\
\hline Skin-1 & 0 & 0.015 & 75.86 & 0.014 & 93.10 & 0.020 & 100 & 0.021 \\
\hline Skin-2 & 0 & 0.015 & 75.86 & 0.013 & 93.10 & 0.019 & 100 & 0.020 \\
\hline Skin-3 & 0 & 0.016 & 75.86 & 0.015 & 93.10 & 0.021 & 100 & 0.022 \\
\hline
\end{tabular}

Figure 7-17 gathers a graphical analysis of the performance improvement obtained thanks to the latency-based optimization. Specifically, for each hyperspectral image, represented in the horizontal axis, the performance -in hyperspectral images processed per second- is shown for two cases: when no timing information is considered (LoopIter-0), and once every actor execution time has been characterized (LoopIter-3). Based on these performance values, the speedup achieved is displayed in blue. Additionally, the performance objectives associated to each working mode presented in Section 7.1 are depicted as full, dashed and pointed horizontal lines performance thresholds- for Fast, Normal and Slow working modes, respectively.

Regarding the speedup achieved, it can be observed that for Brain images it is always below $1.1 \times$, while for Skin images it reaches $1.4 \times$. As explained before, this improvement is obtained when updating actor timing information. Therefore, the speedup obtained is tightly related with the difference between the default actor timing considered by PREESM (100 $\mu \mathrm{s})$ and the updated timing retrieved using PAPIFY.

Comparing the performances with the objective ones associated to the different working modes, some interesting facts can be extracted. First, in the case of the Brain images, once the implementation has been optimized in terms of latency, there is only one case in which the performance cannot be achieved, namely Brain-1-F. On the Skin field, on the contrary, all the performance objectives can be achieved for every image after the optimization. It should be highlighted that reaching the performance of the Fast mode, in most cases, is just possible thanks to the timing information provided by PAPIFY, since in LoopIter- 0 -where no timing data are considered- the performance objective associated to this mode is achieved only for Brain-3 image. 


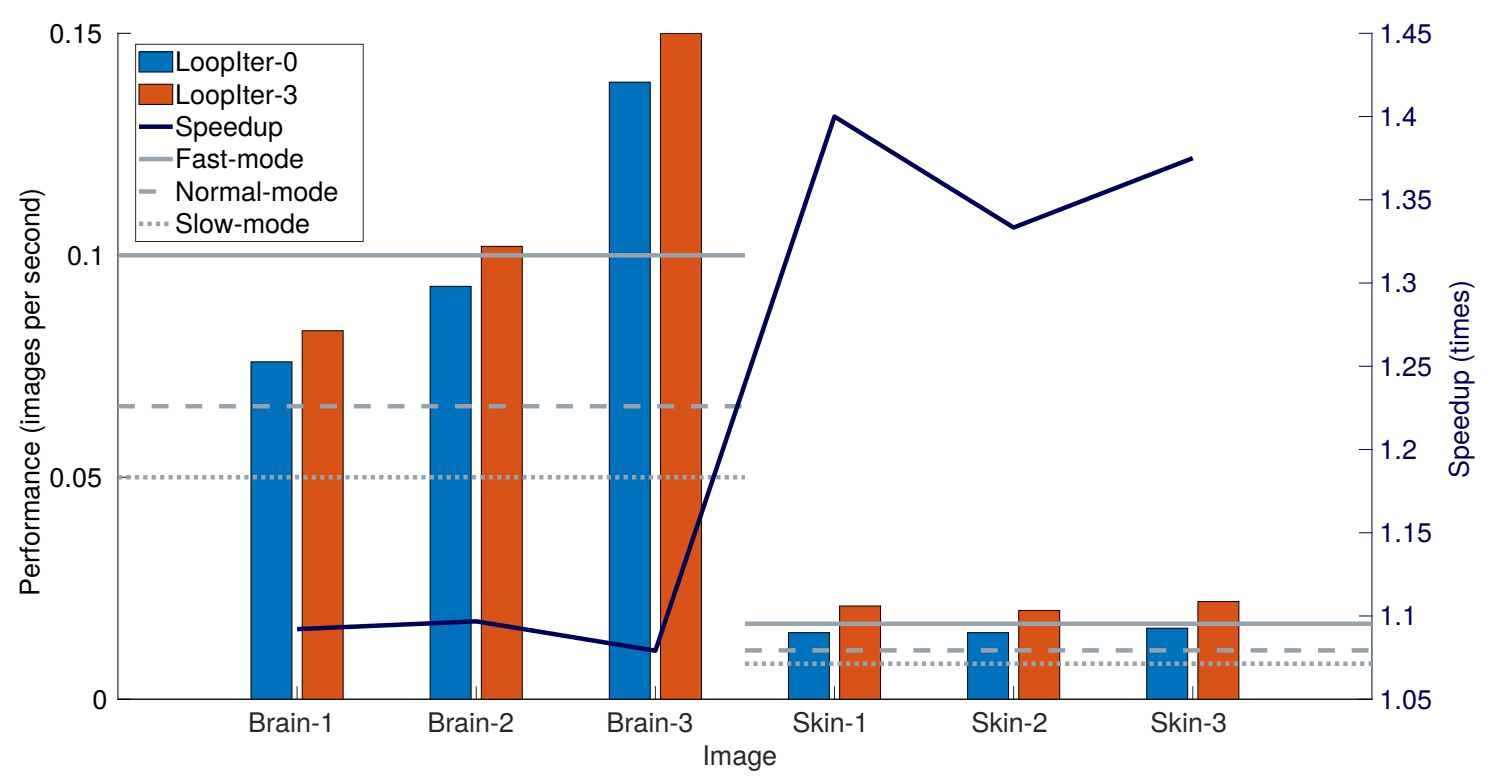

Figure 7-17: Design time latency-based optimization loop results. Analysis of the speedup obtained and comparative with the performance objective for the different working modes

Once the system has been optimized in terms of latency using PAPIFY timing information to enhance PREESM mapping/scheduling decisions, in the next section energy constraints will be included so as to generate systems that are efficient, from the point of view of energy consumption, for each working mode. It should be noted that the timing information extracted during this analysis will also be used in the energyaware optimization loop in design time, since the procedure aims at optimizing the energy consumption while fulfilling performance objectives.

\subsubsection{Energy-aware optimization loop}

Once the optimization loop has been used to optimize the application in terms of latency, this section focuses on the results obtained when including energy-awareness among the system requirements. In order to do so, two steps are required to apply the design time energy-aware optimization loop: to include the energy consumption model in PREESM and to characterize the application in terms of energy consumption.

On the one hand, to provide PREESM with the energy consumption model information, the MPPA model developed in Chapter 5 has been included within the PREESM scenario information. As already introduced in Section 6.3, this model is included as shown in Figure 6-9 and Figure 6-10.

On the other hand, in order to include the energy consumed when the application is running onto the architecture, which is associated with the Energy actors $\leftrightarrow P E$ type section shown in Figure 6-9, the PAPI events composing the computation part of the energy consumption model are monitored through PAPIFY. Similar to the timing profiling carried out in the previous section, in this procedure, the information per 


\section{Energy}

- Energy file path

Enter an excel file (.xls) or a .papify path that contains energy with task names as rows and operator definitions as columns. The Energy actors <-> PE type section will be updated accordingly. Additionally, if a papify-output folder is selected, the model from PAPIFY tab will be applied to parse energy values

\begin{tabular}{|l|l|l|}
\hline Edit file & Refresh \\
\hline Browse Export & Import papify-output \\
\hline
\end{tabular}

\section{Performance objective}

This section contains the performance objective to reach during the energy-aware mapping/scheduling

\begin{tabular}{|l|l|}
\hline Parameter & Value \\
\hline Objective - in executions per second: & 50.0
\end{tabular}

\section{Platform power consumption}

This section includes all the information related to the static power consumed by the platform. Specifically, "Base" represents the baseline of power and, the rest represents the power consumption increment when activating one new PE of a specific type

\begin{tabular}{|l|l|}
\hline Operator Definition & Associated power [W] \\
\hline Base & 10.776 \\
\hline MPPA2Cluster & 0.257 \\
\hline MPPA2IO & 0.0 \\
\hline
\end{tabular}

\section{Communication energy consumption}

This section includes all the information related to the energy consumption due to data communication. Specifically, the energy required to transmit 1 byte from 1 type of PE to another type of PE [p]]

\begin{tabular}{|l|l|l|}
\hline Source I Destination & MPPA2Cluster & MPPA2IO \\
\hline MPPA2Cluster & 118021.42 & 121743.8 \\
\hline MPPA2IO & 126688.71 & 130411.09 \\
\hline - Energy actors $\leftrightarrow$ PE type & & \\
\hline
\end{tabular}

This section includes all the information related to energy consumption of each actor when being executed on each type of processing element. MPPA2Cluster

\begin{tabular}{|l|l|}
\hline Actors & Value \\
\hline top_HSI_cancer_detection/Read_HS_image & 1.0 \\
\hline top_HSI_cancer_detection/Write_results & 1.0 \\
top_HSI_cancer_detection/HSI_cancer_detection/TransposeMatrix & 1.0 \\
top_HSI_cancer_detection/HSI_cancer_detection/DimensionalityReduction/WritePcaResults & 1.0 \\
top_HSI_cancer_detection/HSI_cancer_detection/SpatialSpectralFiltering/DecisionMaking & 1.0
\end{tabular}

Figure 6-9 Energy tab in PREESM scenario (repeated from page 114 


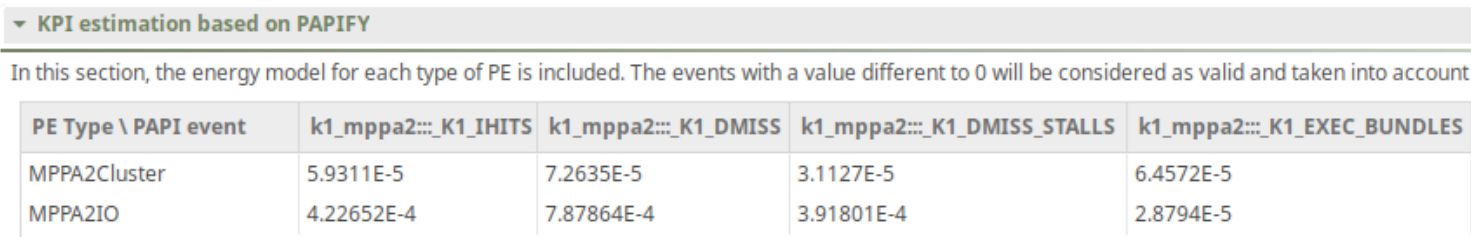

Export model

Figure 6-10: Energy consumption computation model table in PREESM interface (repeated from page 115

actor extracted in each optimization loop iteration is composed of 4 PAPI events. Specifically, as stated in Subsection 5.6.3, the events being monitored are _K1_IHITS, _K1_DMISS, _K1_DMISS_STALLS and_K1_EXEC_BUNDLES. Combining these PAPI events and the energy consumption model of the MPPA computational contribution, the energy consumed by each application actor when being executed on each MPPA resource is included within PREESM scenario information.

After updating all the information related to energy consumption, i.e., MPPA power consumption and the energy consumed by each actor when running on each type of $\mathrm{PE}$, the energy-aware tuning algorithm can be launched via PREESM code generation process. As stated in Section 6.3, the user needs to configure the energy-aware tuning algorithm and to determine the performance objective.

In the case of the configuration, for this study, it has been set to start using half of the resources (Middle option as the starting point) and to search using a binary protocol (Binary option in the searching mode). This configuration will provide a result after evaluating up to 5 different mappings/schedulings.

Regarding the performance objective, it represents the target graph iterations per second that should be reached by PREESM simulation. Specifically, in Section 7.1. the performance objectives (Perf $\left[\frac{\text { images }}{\mathrm{s}}\right]$ ) are defined as hyperspectral images processed per second. In PREESM (Per $\left.f_{P R E E S M}\right)$, in turn, they are adapted following Equation 7-4, so as to provide an equivalent number of images processed per second but considering that, when the image is divided in numTensors tensors, numTensors iterations are needed to process the whole hyperspectral image.

$$
\operatorname{Perf} f_{P R E E S M}\left[\frac{\text { tensors }}{\mathrm{s}}\right]=\operatorname{Perf}\left[\frac{\text { images }}{\mathrm{s}}\right] * \text { numTensors }
$$

The last information that needs to be included within PREESM is the data transmission speed. Since the MPPA implementation relies on the asynchronous communication protocol provided by Kalray, the DMAs that are in charge of the different communications cannot be explicitly controlled by PREESM generated code. The main drawback of this approach, from the point of view of PREESM modeling, is that the number of DMAs involved in the communication vary depending on the number of CC $\$$ being transmitting/receiving in parallel, which is basically translated 
into parallelism in the communication. This behavior, which implies increasing or decreasing the total bandwidth of the NoC depending on the number of active $\mathrm{CC} s$, cannot be specified within PREESM, where the communication speed has to be fixed before launching the code generation.

In the case of study of this $\mathrm{PhD}$, selecting the MPPA communication speed is an important aspect of the system modeling, since, at design time, the energy-aware tuning algorithm uses this value to estimate whether the performance objective is reached or not. This performance is computed as a combination of actor timing (provided by PAPIFY, as shown in Subsection 7.3.3) and time spent transmitting data. To tackle this problematic, the communication speed has been fixed to its most restrictive value; hence, when the number of active $\mathrm{CC} s$ grows and the speed increases on the MPPA PREESM will underestimate the reached performance and will increase the number of involved resources. By doing so, it is ensured that the performance objective will be reached by means of performing conservative performance estimations. The selected communication speeds are shown in Figure 7-20. To be precise, this value has been set to 2GB (2000 bytes transmitted every $\mu$ s in the figure), which is the transmission speed when using only 1 DMA of the $\mathrm{IO}$.

- Memcopy speeds
\begin{tabular}{|l|l|l|}
\hline Each operator definition can be linked to a couple of values (memcpySetupTime, memcpySpeed) respectively setting a time to initiate a memcopy and a speed \\
\hline operator definition & memcpySetupTime & memcpySpeed \\
\hline MPPA2Cluster & 1 & 2000.0 \\
MPPA2IO & 1 & 2000.0 \\
\hline
\end{tabular}

Figure 7-20: MPPA data transmission speed on PREESM

The first aspect to be analyzed in the design time energy-aware optimization loop is the time required by PREESM to generate the application. When using the energy-aware tuning algorithm in the DSE step, PREESM simulates several mappings/schedulings, which implies an increment of this time. Specifically, this increment is proportional to the number of mappings/schedulings analyzed in the process, ranging from 3 to 4 times the one required when optimizing only latency.

The implementations obtained using the energy-aware optimization loop have been characterized in terms of performance and energy consumption. To do so, for each validation experiment configuration Table 7-4, the optimized implementation is executed on the MPPA without any PAPIFY instrumentation. In order to properly compute the application average performance, the applications have been configured to run during 1,000 iterations; hence, since the maximum number of tensors is 50 for Skin experiments-, every hyperspectral image will be processed at least 20 times. Additionally, with this configuration, the application will be iteratively running during enough time to measure the MPPA power consumption, following the procedure used during the development of the energy consumption model (Chapter 5).

These results are gathered in Table 7-11, where the estimation (Estimation) and the real measurements (Measurement) of both performance-in images per second- (Perf) and average power consumption -in W-(Power) are compared. In order to evaluate the 
correctness of the estimations, the relative errors, in percentage, (Errors) are displayed for performance (Perf) and average power consumption (Power). Additionally, to check if the code generation is properly fulfilling the performance objective, the value of each specific working mode (Perf objective) is also shown in hyperspectral images processed per second, which is the one presented in Section 7.1. Additionally, in the Estimation column, a configuration with a certain number of active MPPA resources (Active CCs) is proposed for each image-objective pair.

Table 7-11: Performance (in hyperspectral images processed per second) and power consumption (in W) results. Design time energy-aware tuning using PREESM

\begin{tabular}{|c|c|c|c|c|c|c|c|c|}
\hline \multicolumn{2}{|c|}{ Configuration } & \multicolumn{3}{c|}{ Estimation } & Measurement & \multicolumn{2}{c|}{ Errors } \\
\hline Experiment & $\begin{array}{c}\text { Perf } \\
\text { objective }\end{array}$ & $\begin{array}{c}\text { Active } \\
\text { CCs }\end{array}$ & Perf & Power & Perf & Power & Perf & Power \\
\hline Brain-1-F & 0.1 & 16 & 0.049 & 15.172 & 0.077 & 15.347 & -57.317 & -1.154 \\
\hline Brain-1-N & 0.066 & 16 & 0.049 & 15.172 & 0.077 & 15.302 & -57.354 & -0.853 \\
\hline Brain-1-S & 0.05 & 16 & 0.049 & 15.172 & 0.077 & 15.325 & -57.319 & -1.004 \\
\hline Brain-2-F & 0.1 & 16 & 0.069 & 15.205 & 0.104 & 15.467 & -50.854 & -1.728 \\
\hline Brain-2-N & 0.066 & 10 & 0.067 & 13.655 & 0.097 & 14.072 & -45.815 & -3.050 \\
\hline Brain-2-S & 0.05 & 2 & 0.053 & 11.562 & 0.062 & 11.990 & -16.761 & -3.706 \\
\hline Brain-3-F & 0.1 & 16 & 0.100 & 15.142 & 0.151 & 15.233 & -50.284 & -0.597 \\
\hline Brain-3-N & 0.066 & 2 & 0.080 & 11.512 & 0.094 & 11.906 & -18.270 & -3.424 \\
\hline Brain-3-S & 0.05 & 1 & 0.067 & 11.234 & 0.066 & 11.545 & 1.305 & -2.770 \\
\hline Skin-1-F & 0.017 & 16 & 0.013 & 15.367 & 0.019 & 15.564 & -47.790 & -1.281 \\
\hline Skin-1-N & 0.011 & 3 & 0.011 & 12.011 & 0.014 & 12.448 & -23.116 & -3.631 \\
\hline Skin-1-S & 0.008 & 2 & 0.010 & 11.698 & 0.012 & 12.104 & -21.189 & -3.469 \\
\hline Skin-2-F & 0.017 & 16 & 0.013 & 15.378 & 0.018 & 15.494 & -36.253 & -0.760 \\
\hline Skin-2-N & 0.011 & 3 & 0.011 & 12.020 & 0.013 & 12.409 & -15.670 & -3.237 \\
\hline Skin-2-S & 0.008 & 2 & 0.010 & 11.704 & 0.011 & 12.087 & -14.914 & -3.270 \\
\hline Skin-3-F & 0.017 & 16 & 0.014 & 15.416 & 0.020 & 15.614 & -37.068 & -1.284 \\
\hline Skin-3-N & 0.011 & 3 & 0.012 & 12.052 & 0.014 & 12.469 & -14.929 & -3.462 \\
\hline Skin-3-S & 0.008 & 2 & 0.010 & 11.728 & 0.012 & 12.122 & -14.381 & -3.361 \\
\hline
\end{tabular}

As can be observed, the energy-aware optimization loop included within PREESM provides implementations using different number of MPPA resources CCs- depending on the performance objective and the image being used. On the performance side, Brain-1-F is the only experiment in which the performance objective is not reached and, in this case, the energy-aware protocol generates the system with the highest possible performance. Additionally, analyzing the error in the performance estimations, it can be seen that the higher the number of active CC $\beta$ is, the higher the error becomes, reaching up to a $57 \%$ in the Brain-1-F experiment. This error trend is the one produced by the decision of setting the transmission speed of the MPPA to 2GB. By doing so, the performance of the configurations using more than 1 DMA in the real hardware are underestimated in PREESM. This conservative decision provides systems where the estimation does not fulfill the performance requirement while the real execution does, as in Brain-2-F and Skin-1-F, for example. 
From the point of view of the energy consumption, the methodology provides the configuration that fulfills the performance objective while presenting the lowest average power consumption, which is equivalent to the lowest energy consumption in a streaming-oriented system. To estimate the power/energy consumption of each configuration, PREESM considers all the types of contribution highlighted in Chapter 5, which means that it considers 1) the power consumed by each active MPPA resource, 2) data transmissions and 3) the energy consumed by each actor when being executed on each type of MPPA resource. To be precise, an estimation of the average power consumption of the resulting system is computed, that is, the energy consumed per second by the system. This estimation is carried out once the PREESM mapping/scheduling has been performed, as knowing which resource is executing each actor is required. Consequently, the power/energy consumption estimation reaches a maximum error of $3.62 \%$ (Brain-2-S), which means that the procedure has an accuracy of $96.3 \%$ in a real application. This high accuracy allows, in this case, PrEESM. to generate efficient systems from the point of view of the power/energy consumption.

In order to get deeper into the energy-awareness tuning procedure embedded within PREESM system generation, as an example, the Brain-2- $N$ experiment is represented in Figure 7-21. In this example, a performance objective of 0.666 hyperspectral images per second -represented as a horizontal blue line- is considered. This performance objective is taken into account by the energy-awareness tuning mechanism as a threshold indicating whether it has to increase or decrease the number of active CCs -red points-. As a result, a system using $10 \mathrm{CC} s$ is generated, which is the configuration reaching the objective with the lowest energy consumption. To reach this solution, as can be seen, 4 searching points have been analyzed, represented as numbered green circles. For each point, the estimations of both performance (vertical axis) and power/energy consumption (horizontal axis) are shown.

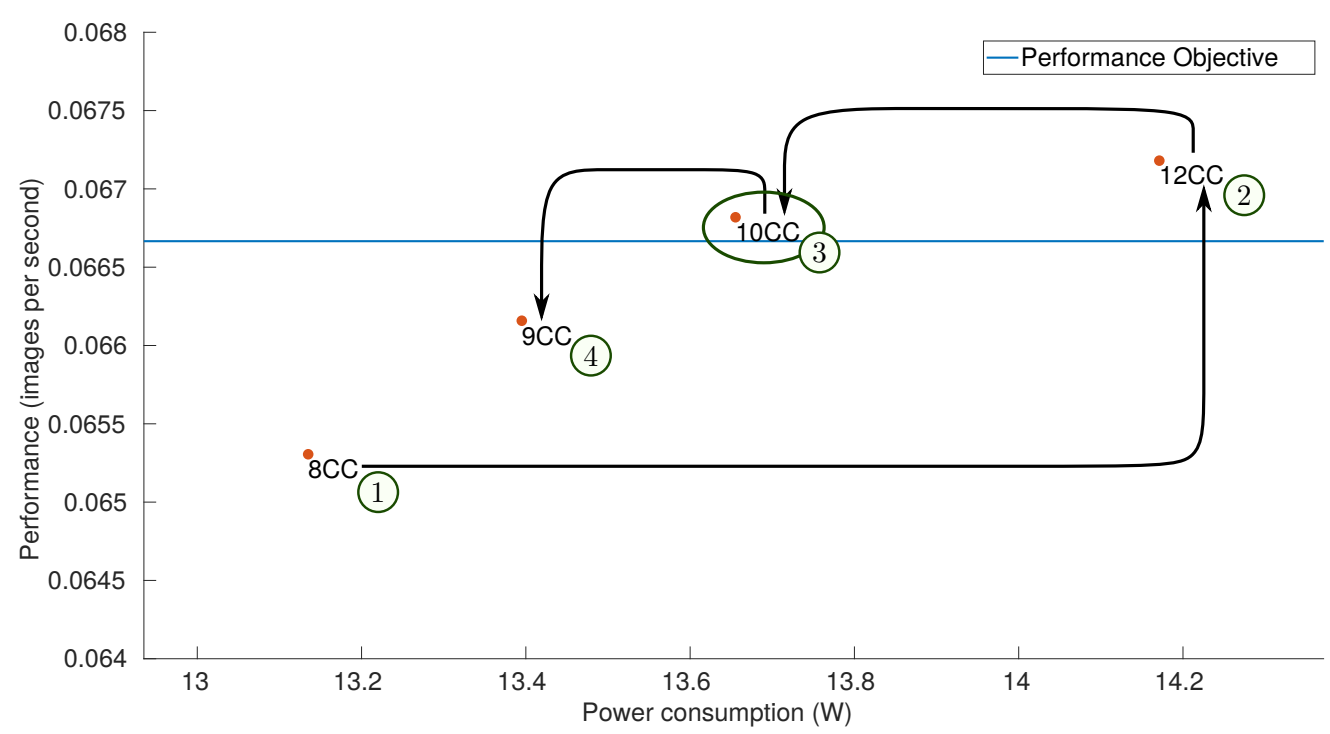

Figure 7-21: PREESM energy-awareness tuning real example. Brain-2- $N$ experiment 
Finally, the last step is to compare the systems obtained using the design time energy-aware optimization loop with the system done by hand. Specifically, Table 7-12 collects the results of Table 7-7, where the Implementation by hand has been evaluated in terms of performance, Perf, -in hyperspectral images processed per second- and Power consumption -in W- for the different Experiments, i.e., for each hyperspectral image and working mode combination. Additionally, the system configurations obtained using the Design time energy-aware optimization loop shown in Table 7-11 are also collected in this table so as to compare both results. Specifically, the values measured (not estimated) for both performance and power/energy consumption are shown, since the comparison is fairer when the real measurements are used. The evaluation of the results is twofold: first, the validity of the system generated by the design time energy-aware optimization loop is considered, being valid if and only if the performance objective, Perf objective, is reached. Furthermore, for those experiments that are considered valid $(\checkmark)$, the power/energy savings of the energy-aware implementation, when compared to the implementation by hand, Power savings, are displayed.

Table 7-12: Performance (images per second), power (W), and power savings (\%) of the HSI processing chain for the validation experiments. Implementation by hand vs design time energy-aware optimization loop. An experiment is valid if it reaches the performance objective

\begin{tabular}{|c|c|c|c|c|c|c|c|}
\hline \multirow[b]{2}{*}{ Experiment } & \multicolumn{2}{|c|}{$\begin{array}{c}\text { Implementation } \\
\text { by hand }\end{array}$} & \multicolumn{2}{|c|}{$\begin{array}{c}\text { Design time } \\
\text { energy-aware }\end{array}$} & \multicolumn{3}{|c|}{ Evaluation } \\
\hline & Perf & Power & Perf & Power & $\begin{array}{c}\text { Perf } \\
\text { objective }\end{array}$ & Valid & $\begin{array}{l}\text { Power } \\
\text { savings }\end{array}$ \\
\hline Brain-1-F & \multirow{3}{*}{0.357} & \multirow{3}{*}{16.18} & 0.077 & 15.347 & 0.1 & $x$ & - \\
\hline Brain-1-N & & & 0.077 & 15.302 & 0.066 & $\sqrt{ }$ & 5.43 \\
\hline Brain-1-S & & & 0.077 & 15.325 & 0.05 & $\checkmark$ & 5.29 \\
\hline Brain-2-F & \multirow{3}{*}{0.392} & \multirow{3}{*}{15.96} & 0.104 & 15.467 & 0.1 & $\checkmark$ & 3.09 \\
\hline Brain-2-N & & & 0.097 & 14.072 & 0.066 & $\checkmark$ & 11.83 \\
\hline Brain-2-S & & & 0.062 & 11.990 & 0.05 & $\sqrt{ }$ & 24.87 \\
\hline Brain-3-F & \multirow{3}{*}{0.567} & \multirow{3}{*}{15.24} & 0.151 & 15.233 & 0.1 & $\checkmark$ & 0.05 \\
\hline Brain-3-N & & & 0.094 & 11.906 & 0.066 & $\checkmark$ & 21.88 \\
\hline Brain-3-S & & & 0.066 & 11.545 & 0.05 & $\sqrt{ }$ & 24.25 \\
\hline Skin-1-F & \multirow{3}{*}{0.090} & \multirow{3}{*}{17.28} & 0.019 & 15.564 & 0.017 & $\checkmark$ & 9.93 \\
\hline Skin-1-N & & & 0.014 & 12.448 & 0.011 & $\sqrt{ }$ & 27.97 \\
\hline Skin-1-S & & & 0.012 & 12.104 & 0.008 & $\sqrt{ }$ & 29.96 \\
\hline Skin-2-F & \multirow{3}{*}{0.090} & \multirow{3}{*}{17.32} & 0.018 & 15.494 & 0.017 & $\checkmark$ & 10.54 \\
\hline Skin-2-N & & & 0.013 & 12.409 & 0.011 & $\sqrt{ }$ & 28.35 \\
\hline Skin-2-S & & & 0.011 & 12.087 & 0.008 & $\checkmark$ & 30.21 \\
\hline Skin-3-F & \multirow{3}{*}{0.090} & \multirow{3}{*}{17.35} & 0.020 & 15.614 & 0.017 & $\checkmark$ & 10.00 \\
\hline Skin-3-N & & & 0.014 & 12.469 & 0.011 & $\sqrt{ }$ & 28.13 \\
\hline Skin-3-S & & & 0.012 & 12.122 & 0.008 & $\checkmark$ & 30.13 \\
\hline
\end{tabular}

Focusing on the energy consumption of this analysis, Figure 7-22 graphically compares the energy consumption of the implementation done by hand with the 
measurements obtained using the energy-aware optimization loop. The energy consumption values are represented using bars and associated to each hyperspectral image, which are distributed along the horizontal axis. Additionally, the power/energy savings are displayed using full, dashed and pointed green lines for Fast, Normal and Slow working modes for the experiments that fulfill the performance objective.

From this analysis, it can be concluded that the more relaxed the performance objective is (Slow working mode), the larger energy consumption savings are obtained. Specifically, thanks to the energy-aware optimization loop implemented within PREESM, energy savings up to $30 \%$ (in Slow working mode) can be achieved following an automatic system generation.

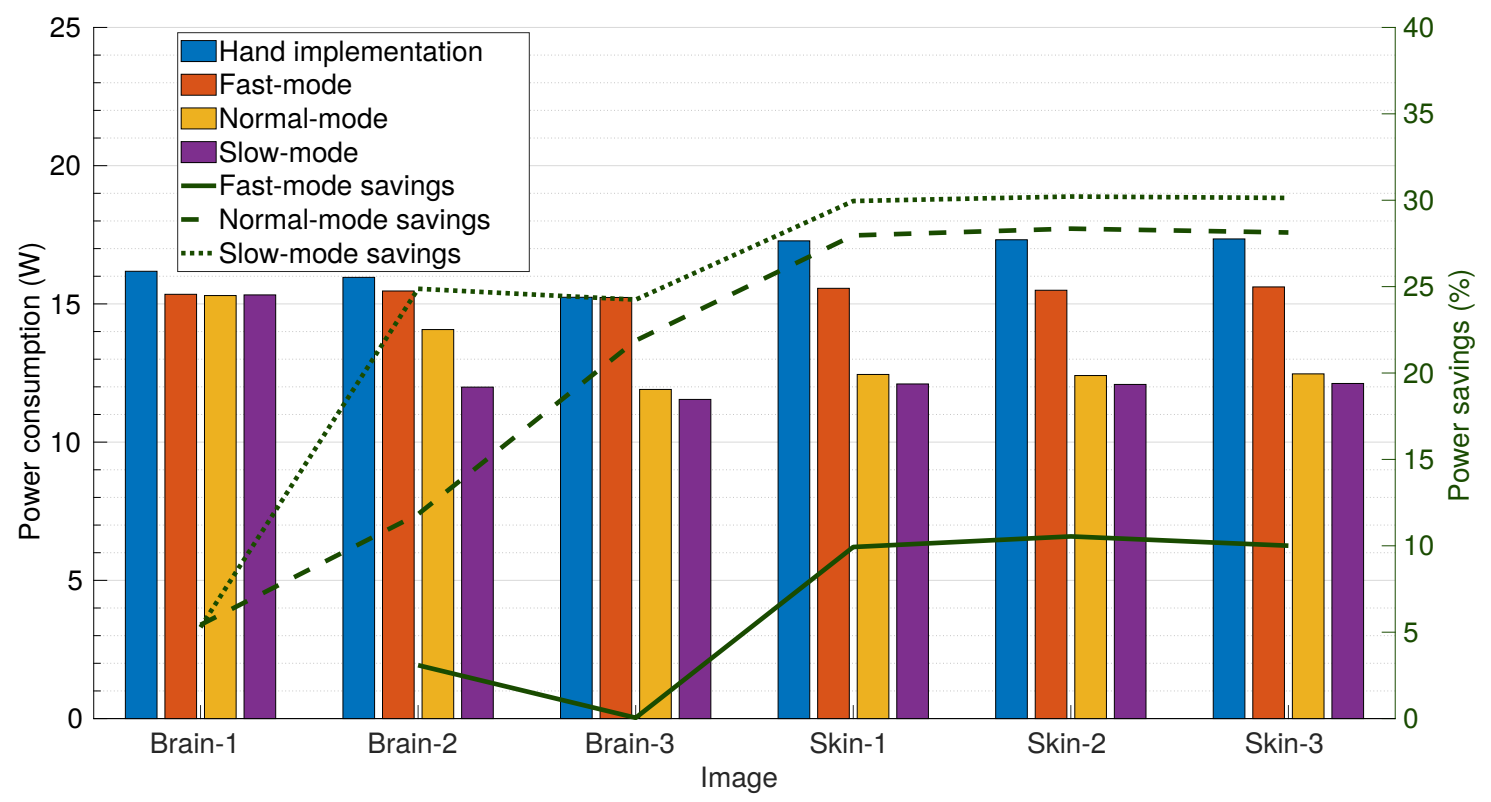

Figure 7-22: Design time energy-aware optimization loop results. Analysis of the energy savings obtained for the different working modes

Although the increment in the time required to generate the system might be considered as a drawback, PREESM now supports the automatic generation of efficient system implementations where the number of active resources is automatically selected to minimize the power/energy consumption while fulfilling a performance objective. Even though the maximum performance is around 6 times lower than the one that can be obtained with the implementation done by hand, this new feature can achieve up to $30 \%$ energy savings, which can be especially useful when the target application runs, for example, on a battery-supplied system. 


\subsection{Runtime optimization}

In contrast to the previous section, where the design time optimization has been addressed, this part of the analysis focuses on the runtime energy-aware optimization of dataflow applications using SPiDER. To perform this analysis, the dataflow version of the application implementation presented in Subsection 7.3.2 has been adapted to include parameters changing at runtime. This new version is presented in Subsection 7.4.1. Afterwards, the implementations obtained using the standard SPiDER optimization loop, which focuses on optimizing latency, are evaluated with and without PAPIFY using the $\mathrm{x} 86$ architecture in Subsection 7.4.2. Later, in Subsection 7.4.3, energy-awareness is included within the previous experiments, together with a performance objective, to analyze SPiDER behavior when using the runtime energy-aware optimization loop. Finally, as introduced in Section 7.1, the loop is simulated in Subsection 7.4.4 using the MPPA architecture. It is worth pointing out that, as explained in Section 7.1, since this validation is carried in two steps, the experiments related with latency-based optimization and runtime energy-aware optimization loop are analyzed using an x86 architecture. After this, the loop is simulated on the MPPA to validate the procedure using the energy consumption model developed in Chapter 5 .

\subsubsection{Dataflow implementation with dynamism}

In the context of HSI cancer detection, some dynamic behaviors may appear. For example, the spatial/spectral resolution of the hyperspectral image that needs to be processed may change if the analysis is done considering regions of interest. Another example can be linked to the available time slot to execute the supervised classification algorithm that has been selected as this PhD use-case. As mentioned in Section 7.1. this algorithm is executed in parallel with an unsupervised classification block, whose execution time has been used to set the performance objective. In this regard, a situation in which the unsupervised classification algorithm changes at runtime can be foreseen; hence, the available time slot to execute the supervised classification algorithm may vary, which is translated into the performance objective dynamically changing at runtime.

To tackle this variability, and starting from the dataflow specification of the HSI cancer detection processing chain explained in Subsection 7.3.1, the required modifications to include runtime dynamism are introduced in this section. To react to these changes, the dataflow graph itself changes and the number of actors that SPiDER has to execute increases/decreases dynamically. Consequently, the workload associated to each actor may also vary, which is the ideal scenario to validate the monitoring infrastructure proposed in this PhD, PAPIFY.

In order to specify dynamism in the dataflow graph, a special configuration actor called ConfigSelector has been included within the network, as shown in Figure 7-23. 
This actor is continuously waiting for an event that changes the expected behavior of the application. In this specific implementation, a change in the spatial/spectral resolution is simulated by changing the image selected from the dataset, while a change in the available time slot is simulated by changing the working mode. Once this event is triggered, it generates the values of the dynamic parameters that have been included within the new dataflow version of the application. Specifically, these parameters, which are the ones previously introduced in Table 7-8, 1) inform Read_HS_image and Write_results actors of the image to be read; 2) change both spatial and spectral resolutions (columns, rows and bands parameters); 3) adapt the number of tensors considering the new image (numTensors); 4) set up the parallelism of the dataflow application with the values gathered in Table 7-9 and 5) define the performance objective, i.e., the selected working mode.

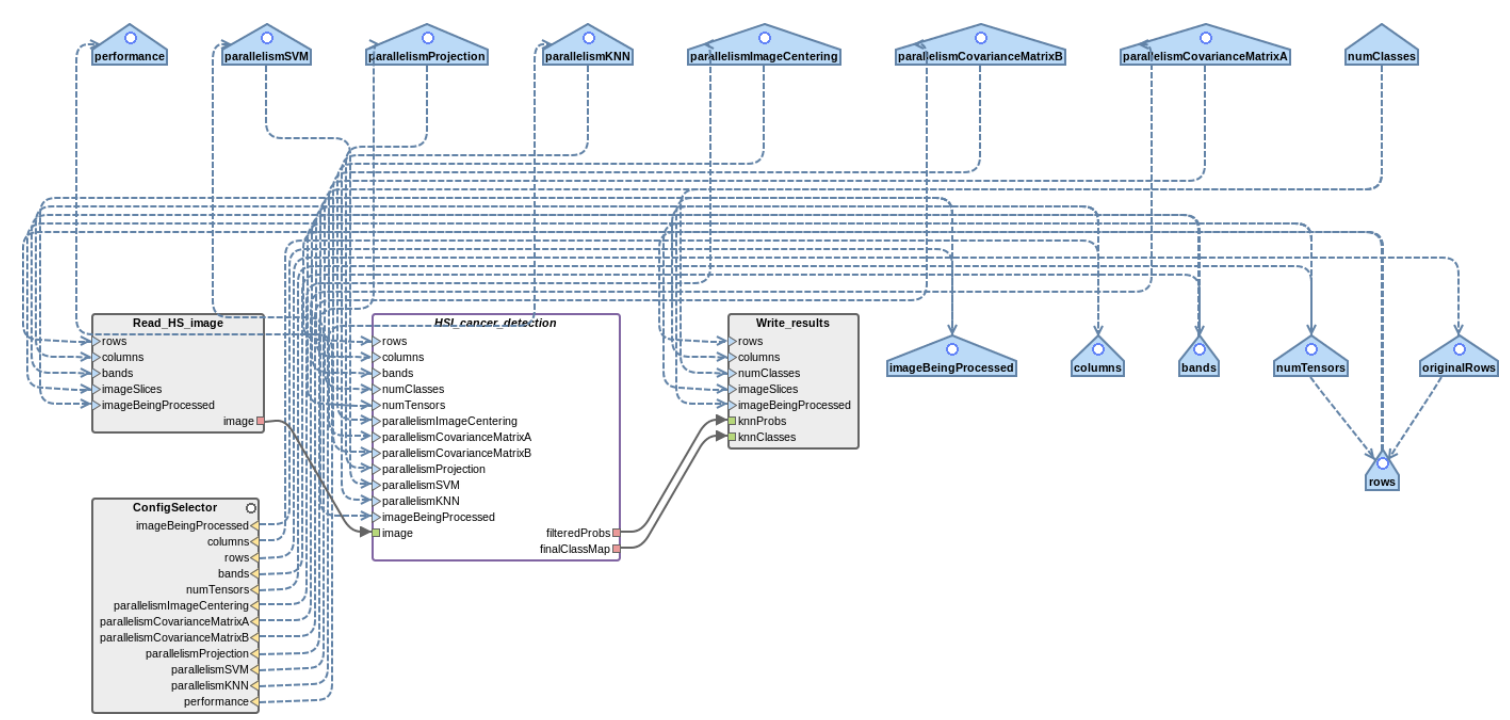

Figure 7-23: SPiDER $\mid$ HSI processing chain dataflow implementation with dynamic parameters

As a result, after including the new configuration actor, the system will adapt its behavior considering the spatial and spectral resolutions of the image. Then, when including energy-awareness, the available execution time slot may also change, hence varying the performance objective.

\subsubsection{Latency-based application optimization}

Similarly to the study carried out in Subsection 7.3.3, where PREESM dataflow implementation has been characterized when optimizing latency at design time with and without the use of PAPIFY, in this section, the equivalent experiments are carried out using SPiDER. Nevertheless, as this study focuses on runtime latency-based optimization, when using PAPIFY, direct timing feedback will be provided to SPiDER GRT. By doing so, the system will be self-aware of the time required to execute each actor and, thus, the system will be automatically optimized with on-the-fly data. 
It is worth pointing out that, as already mentioned Section 7.1, this set of experiments is performed on a multicore architecture that gathers 16 x86 cores. This platform runs at $2.4 \mathrm{GHz}$ and uses a shared memory block for all its processors. These characteristics differ from the MPPA ones, since it runs at $550 \mathrm{MHz}$ and the memory is distributed among the $16 \mathrm{CC}$. For these reasons, the performance results obtained in this analysis are expected to outperform the ones obtained for the MPPA architecture.

Table 7-13 gathers the results associated to the latency-based optimization. In this table, performance results (in terms of hyperspectral images analyzed per second) are shown without PAPIFY usage (No PAPIFY) and enabling PAPIFY timing feedback (PAPIFY feedback). Additionally, the speedup (Speedup) achieved thanks to the extra timing information is also displayed. As can be seen, although PAPIFY instrumentation would intuitively lead to a performance detriment, the obtained performance improvements overcome this drawback.

Table 7-13: SPiDER latency optimization using PAPIFY timing feedback

\begin{tabular}{|c|c|c|c|}
\hline Image & No PAPIFY & PAPIFY feedback & Speedup (times) \\
\hline Brain-1 & 0.242 & 0.280 & 1.158 \\
\hline Brain-2 & 0.317 & 0.323 & 1.019 \\
\hline Brain-3 & 0.498 & 0.545 & 1.093 \\
\hline Skin-1 & 0.046 & 0.061 & 1.338 \\
\hline Skin-2 & 0.047 & 0.060 & 1.294 \\
\hline Skin-3 & 0.047 & 0.061 & 1.304 \\
\hline
\end{tabular}

Figure 7-24 analyzes the results showing for each image (horizontal axis) both performances; additionally, the speedup obtained is plotted. As can be seen, although the architecture is different, the speedups follow the same trend observed during the counterpart analysis done for the design time and the MPPA (Figure 7-17). In other words, when processing Brain images, the speedup obtained is lower than the one achieved when processing Skin images. In this regard, the lowest speedup is $1.019 \times$ (Brain-2) and the highest one is $1.338 \times($ Skin-1). The difference among speedups in this study is linked to the specific execution times of each actor, which are different depending on the hyperspectral image being analyzed. Specifically, the closer the

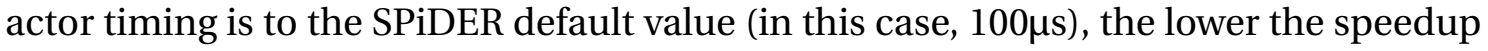
obtained is. For this reason, in bigger images -Skin-\#-, actors have larger workloads; hence, the speedups obtained are higher.

In this section, the benefits of feeding SPiDER GRT with PAPIFY timing information have been shown. This extra information can be used to enhance the decisions taken bySPiDER runtime manager during the mapping/scheduling procedure. In the next sections, energy-awareness will be included within the parameters to take into consideration, feeding the manager with both timing and PMC information so as to achieve efficient system implementations in terms of energy consumption. 


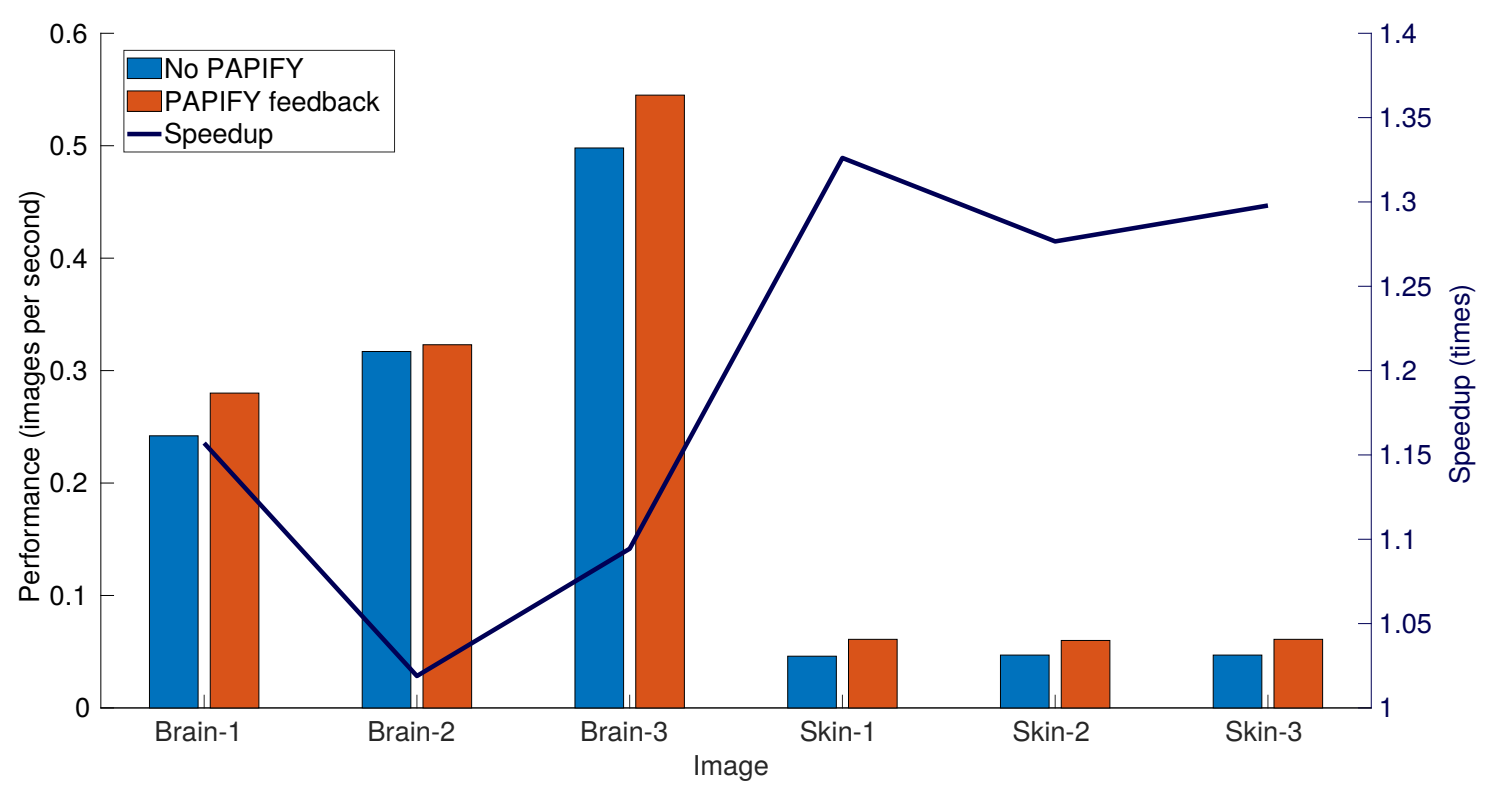

Figure 7-24: Runtime latency-based optimization loop results. Speedup analysis

\subsubsection{Energy-aware optimization loop}

During this part of the analysis, the energy-aware optimization loop embedded within SPiDER runtime is evaluated. In order to do so, as explained in Section 7.1, these experiments are also performed using the aforementioned 16-core x86 architecture. Consequently, in order to have a full closed-loop at runtime, a simulated energy consumption model for this architecture has been used. As explained in Section 7.1. the x86 model does not aim at providing real energy consumption estimations, as this architecture has not been modeled, but to validate the energy-aware tuning algorithm embedded within SPiDER runtime manager. Considering that the only part being architecture-dependent within the energy-aware optimization loop is the energy consumption model, this set of experiments aims at validating the architectureindependent blocks of the optimization loop. Namely, these blocks are 1) the PMCs monitoring done through PAPIFY, 2) the procedure to measure the performance and to estimate the energy consumption (considering the use of a simulated model), 3) the decision mechanism embedded within SPiDER and 4) the reactiveness to dynamic changes in the application parameters.

It should be noted that this analysis must be considered together with the one done in Subsection 7.4.4, where the energy consumption model for the MPPA is characterized with the HSI cancer detection application, hence validating the only architecture-dependent block of the energy-aware optimization loop.

In this regard, two important aspects of this set of experiments must be highlighted before starting the analysis: the experiments order and the performance objectives.

First, in order to test all the image-working mode combinations, only one dynamic parameter will be varied in each configuration change. Specifically, all the images will 
be evaluated in every working mode before changing the mode itself. Additionally, when the working mode changes, the image will be maintained. Summing up, the experiment order will be the one shown in Table 7-14, starting with Brain-1-S experiment (in green) and finishing with Skin-3-F one (in red). In this table, the direction of the arrows represents the next configuration, being a column change a modification in the image being analyzed and a row change the use of a different working mode.

Table 7-14: SPiDER energy-aware optimization experiments order

\begin{tabular}{|c|c|c|c|c|c|c|}
\cline { 2 - 7 } \multicolumn{1}{c|}{} & Brain-1 & Brain-2 & Brain-3 & Skin-1 & Skin-2 & Skin-3 \\
\hline Slow & & & & & & $\longrightarrow$ \\
\hline Normal & & & & & & \\
\hline Fast & $\checkmark$ & & & & & \\
\hline
\end{tabular}

Additionally, aiming at fully characterizing the energy-aware optimization loop, the performance objectives associated to each working mode have been adapted to the higher computational power of the x86 architecture. Compared to the MPPA platform, which runs at $550 \mathrm{MHz}$ and presents a distributed and hierarchical memory layout (NUMA memory architecture), the x86 architecture overtakes it running at $2.4 \mathrm{GHz}$ and using a shared memory block. For these reasons, as demonstrated when comparing the results obtained in Subsection 7.3.3 (for the MPPA) and Subsection 7.4.2 (for the x86 architecture), using the dataflow implementation, the performance achieved by the $x 86$ platform is $3 \times$ the one reached using the MPPA. In this regard, it should be considered that, since the energy consumption estimations for the x86 architecture are computed by means of a simulated model, the power/energy consumption differences between both architectures are not taken into account when modifying the performance objective. This decision has been made so as to focus this section on the evaluation of the energy-aware optimization loop and not on the accuracy of the energy estimation, which will be deeply analyzed in Subsection 7.4.4.

Considering this extra performance, the performance objectives described in Table 7-3 (Objective orig $_{\text {) }}$ have been weighted following Equation 7-5. This equation employs a scale factor to adapt the x86 architecture performance objective $\left(\right.$ Objective $\left._{x 86}\right)$ using 1) the MPPA maximum performances extracted from Table 7-10 $\left(P e r f_{M P P A}\right)$ and 2) the x86 maximum performances extracted from Table 7-13 $\left(P e r f_{x 86}\right)$. Additionally, Table 7-15, gathers the new performance objectives that will be used for the x86 platform. The performances have been adapted for each working mode (Slow for slow mode, Normal for normal mode and Fast for fast mode) and each image applying a scale factor (ScaleFactor) that is computed as $\frac{\operatorname{Per} f_{x 86}}{\operatorname{Per} f_{M P P A}}$.

$$
\text { Objective }_{x 86}=\text { Objective }_{\text {orig }} * \frac{\operatorname{Per} f_{x 86}}{\operatorname{Per} f_{M P P A}}
$$

Once both the experiment order and the performance objectives that will be used in each experiment have been defined, the set of experiments itself can be evaluated. 
Table 7-15: Energy-aware performance objectives adapted for the x86 architecture

\begin{tabular}{|c|c|c|c|c|c|c|}
\hline Image & Perf $_{\text {MPPA }}$ & Perf $_{\mathbf{x 8 6}}$ & Scale Factor & Fast & Normal & Slow \\
\hline Brain-1 & 0.083 & 0.280 & 3.37 & 0.337 & 0.222 & 0.169 \\
\hline Brain-2 & 0.102 & 0.323 & 3.17 & 0.317 & 0.209 & 0.159 \\
\hline Brain-3 & 0.150 & 0.545 & 3.63 & 0.363 & 0.239 & 0.182 \\
\hline Skin-1 & 0.020 & 0.061 & 3.05 & 0.045 & 0.034 & 0.024 \\
\hline Skin-2 & 0.019 & 0.060 & 3.16 & 0.051 & 0.035 & 0.025 \\
\hline Skin-3 & 0.021 & 0.061 & 2.90 & 0.046 & 0.032 & 0.023 \\
\hline
\end{tabular}

To do so, just one execution of the dataflow application using SPiDER is required, since all the changes in the dynamic parameters occur at runtime. Additionally, each execution of the graph iteration is profiled in terms of timing and PMC occurrences per actor using PAPIFY. These data is fed to the SPiDER GRT, which is in charge of 1) estimating the energy consumption, 2) evaluating if the current configuration is the optimal, 3) deciding whether a new configuration has to be tested and 4) computing the new application mapping/scheduling. It should be noted that, differently to the optimization loop at design time, the performances used to decide whether the performance objective is reached or not in this case are measured and not estimated.

To properly characterize the energy-aware optimization loop, it has been decided to run 1,000 iterations of each configuration before triggering a change in the dynamic parameters. By doing so, all the images will be fully processed at least 20 times and the energy-aware tuning algorithm will have more than enough iterations to find the most suitable system configuration. Since the experiments requiring the highest number of tensors (50) are the Skin-\# ones, 50 graph iterations are required to process one full hyperspectral image in these cases.

The results associated with this set of experiments are gathered in Table 7-16. In this table, each row represents the processing of a different image and each group of columns is associated to a different working mode. Additionally, for each working mode, 3 values are shown: 1) the number of active PEs in the x86 architecture, i.e., the enabled cores $(P E s) ; 2)$ the performance achieved for this experiment (Perf) -in hyperspectral images per second-; and 3) the estimated energy consumption (Energy) using the simulated model in J per second, i.e., W. It should be noted that both Perf and Energy values are the ones obtained after the energy-aware optimization loop has selected the optimal configuration.

Nevertheless, in Brain-1-F, the performance objective is not reachable. Consequently, the selected configuration is the one providing the highest performance, i.e., the closest one to the objective, independently of its energy consumption.

In this regard, the last analysis provided in this section focuses on the energy consumption reduction obtained thanks to the energy-aware optimization loop. Although the energy consumption model used in this set of experiments is simulated, theoretical energy consumption optimizations are shown in Table 7-17. To compute these values, the energy consumption of the configurations with the highest 
Table 7-16: SPiDER energy-aware system configurations using the energy-aware tuning algorithm and the x86 16-core architecture

\begin{tabular}{|c|c|c|c|c|c|c|c|c|c|}
\hline \multirow{2}{*}{ Image } & \multicolumn{4}{|c|}{ Slow } & \multicolumn{3}{c|}{ Normal } & \multicolumn{3}{c|}{ Fast } \\
\cline { 2 - 11 } & PEs & Perf & Energy & PEs & Perf & Energy & PEs & Perf & Energy \\
\hline Brain-1 & 4 & 0.219 & 11.810 & 5 & 0.219 & 12.063 & 16 & 0.293 & 14.858 \\
\hline Brain-2 & 2 & 0.192 & 11.292 & 3 & 0.192 & 11.549 & 8 & 0.332 & 12.837 \\
\hline Brain-3 & 1 & 0.201 & 11.066 & 2 & 0.201 & 11.293 & 4 & 0.393 & 11.812 \\
\hline Skin-1 & 2 & 0.033 & 11.291 & 3 & 0.042 & 11.549 & 5 & 0.054 & 12.063 \\
\hline Skin-2 & 2 & 0.033 & 11.291 & 3 & 0.042 & 11.548 & 5 & 0.053 & 12.063 \\
\hline Skin-3 & 2 & 0.033 & 11.291 & 2 & 0.033 & 11.291 & 4 & 0.048 & 11.806 \\
\hline
\end{tabular}

performance has also been estimated, which is shown in Max Energy column. Finally, for each image and each working mode, a pair of values are collected. The former, Energy, is the energy consumption estimation already shown in Table 7-16. The latter, $\%$, is the percentage of energy consumption that is theoretically saved using a different system configuration. In the case of Brain-1-F, the energy savings are not computed, since in this experiment the performance objective is not reached.

Table 7-17: SPiDER energy-aware energy consumption reduction using the energy-aware tuning algorithm and the x86 16-core architecture

\begin{tabular}{|c|c|c|c|c|c|c|c|}
\hline \multirow{2}{*}{ Image } & Max & \multicolumn{2}{|c|}{ Slow } & \multicolumn{2}{c|}{ Normal } & \multicolumn{2}{c|}{ Fast } \\
\cline { 3 - 8 } & Energy & Energy & \% & Energy & \% & Energy & \% \\
\hline Brain-1 & 14.858 & 11.810 & 20.512 & 12.063 & 18.812 & 14.858 & - \\
\hline Brain-2 & 14.859 & 11.292 & 24.003 & 11.549 & 22.272 & 12.837 & 13.605 \\
\hline Brain-3 & 14.810 & 11.066 & 25.279 & 11.293 & 23.746 & 11.812 & 20.244 \\
\hline Skin-1 & 14.769 & 11.291 & 23.548 & 11.549 & 21.806 & 12.063 & 18.324 \\
\hline Skin-2 & 14.746 & 11.291 & 23.429 & 11.548 & 21.688 & 12.063 & 18.196 \\
\hline Skin-3 & 14.876 & 11.291 & 24.099 & 11.291 & 24.098 & 11.806 & 20.639 \\
\hline
\end{tabular}

In Figure 7-25, the power/energy consumption savings are graphically analyzed. In this figure, the estimations obtained using the simulated model are represented using bars -in W- and grouped for each hyperspectral image (horizontal axis). Additionally, the power/energy savings -in \%- obtained for Fast, Normal and Slow working modes are plotted using full, dashed and pointed lines, respectively. As can be seen, energy consumption reductions from $13.605 \%$ (Brain-2-F) to $25.279 \%$ (Brain-3-S) can be achieved using the proposed optimization methodology.

In this section, PMC PAPIFY feedback has been added to the information provided to SPiDER GRT. Considering these extra data, the energy-aware tuning mechanism has been enabled to optimize the system configuration at runtime using the energyawareness optimization closed loop. Although the energy model employed in this section is a simulated model for the x86 architecture, the advantages of having this energy-based optimization procedure have been shown. Additionally, in the next section, the energy consumption model for the MPPA will be thoroughly characterized 


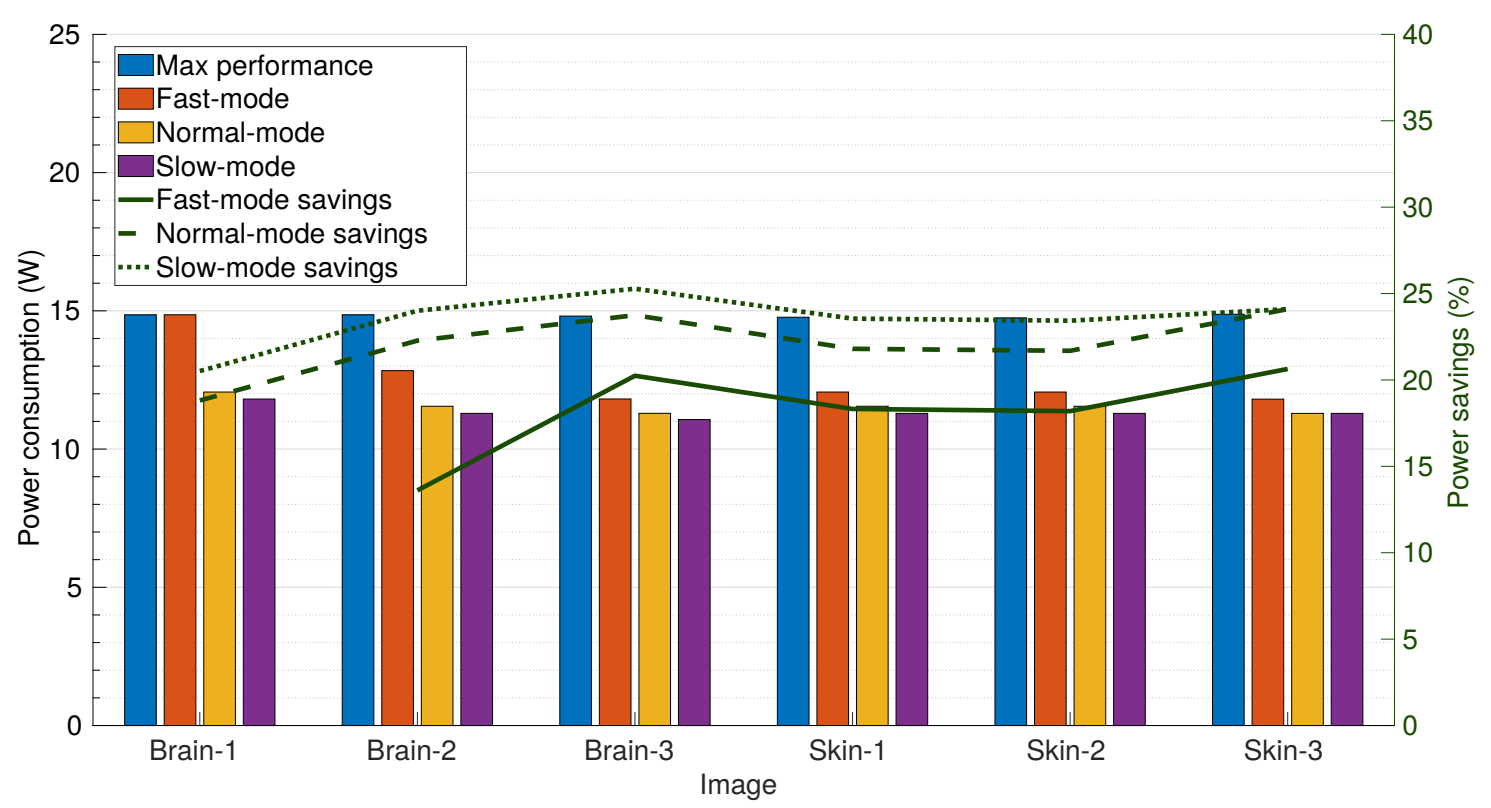

Figure 7-25: Runtime energy-aware optimization loop results for the x86 architecture. Analysis of the energy savings obtained for the different working modes

and an analysis of the real energy consumption reduction that could be achieved using this methodology is carried out on this platform. Taking into consideration that the energy consumption model is the only part being architecture-dependent, it can be easily foreseen that the combination of both can lead to a self-adaptation loop where the energy consumption is minimized using SPiDER on the MPPA.

\subsubsection{Energy-awareness validation on the MPPA}

After the validation of the runtime energy-aware optimization loop presented in the previous section, the objective of this section is to assess the energy consumption model developed in Chapter 5. This evaluation consists in simulating the energyaware tuning algorithm and, for each system configuration, estimating the energy consumption using the real MPPA energy consumption model and the profiling information retrieved when running the application on the real architecture.

Therefore, the set of experiments composing this section is built as follows. First, for each image and each working mode, the system using half of the resources (8CCs) will be generated using PREESM and both the performance and the energy consumption will be measured. After this, the energy-aware tuning algorithm procedure will be applied for each case, increasing/decreasing the number of active CCs depending on whether the performance objective is reached or not. The search will continue until the energy-aware tuning algorithm finds the most suitable solution. In this analysis, each generated system will run on the MPPA during 1,000 graph iterations to properly measure both performance and energy consumption, following the same reasoning explained in Subsection 7.3.4. 
After this, the same procedure will be carried out but, this time, the energy consumption will be estimated (not measured). As a result, both the accuracy of the energy consumption model and the feasibility of embedding a real model within the optimization loop are evaluated. Considering that in the runtime version of the energyaware optimization loop the performance is measured and not estimated, the only value that may differ in both rounds of experiments is the energy consumption.

In this regard, the results associated to the first round of experiments are collected in Table 7-18, In this table, each row corresponds to a different image-working mode pair and linked to a specific performance objective, Perf Obj. Additionally, for each tested configuration, Config-\#, 1) the number of active CCs, CCs, 2) a column showing if the performance is reached $(\boldsymbol{V})$ or not $(\boldsymbol{X})$, Perf and 3) the measured power/energy consumption, Energy, are shown. In these tests, the energy-aware tuning algorithm selects the optimal configuration after testing up to 5 system configurations, i.e., 5 graph iterations. For this reason, and considering the lowest number of tensors used to process one hyperspectral image in this study is 9 (Brain-3 experiment), the optimal configuration will be always reached even before analyzing one complete hyperspectral image and kept during the rest of the execution. Finally, it is worth pointing out that, in Brain-1-F experiment, which is the only one in which a fifth configuration needs to be tested, the performance is not reachable. Therefore, in this situation, the configuration providing the highest performance is selected, independently of its associated energy consumption.

Once the real measurements have been taken, the energy consumption model, developed in Chapter 5, is employed to estimate the energy of each proposed configuration. Figure 7-26 presents a comparison between both energy measurements and its counterpart estimation. To ease the understanding of the figure, the energy consumption measurements presented in Table 7-18 (represented in blue) have been ordered following an ascending order and the associated estimations for each point have been also displayed (in red). This comparison has an average accuracy of $97.61 \%$, while reaching a maximum relative error of $3.62 \%$, which has been circled in the figure. This value is associated to Brain-2-N Config-4 estimation, where 3 CCs are enabled.

Finally, as in the case of the design time energy-aware optimization loop (Subsection 7.3.4), the last step is to compare the implementation done by hand with those systems that would be selected by the runtime energy-aware optimization loop on the MPPA. Specifically, Table 7-19 gathers both the results obtained using the implementation by hand, Implementation by hand, (extracted from Table 7-7) and the ones associated to the runtime energy-aware optimization loop, Runtime energy-awareness, (extracted from Table 7-18, Specifically, measured (not estimated) Perf -in images per second- and Power consumption -in W- are displayed for each type of implementation and each Experiment. Additionally, to evaluate the runtime implementations, Evaluation, a Valid column is included indicating whether the performance objective, Perf objective, is reached or not. Finally, Power savings column indicates the power reduction obtained by the runtime energy-awareness implementation when compared to the implementation by hand. 


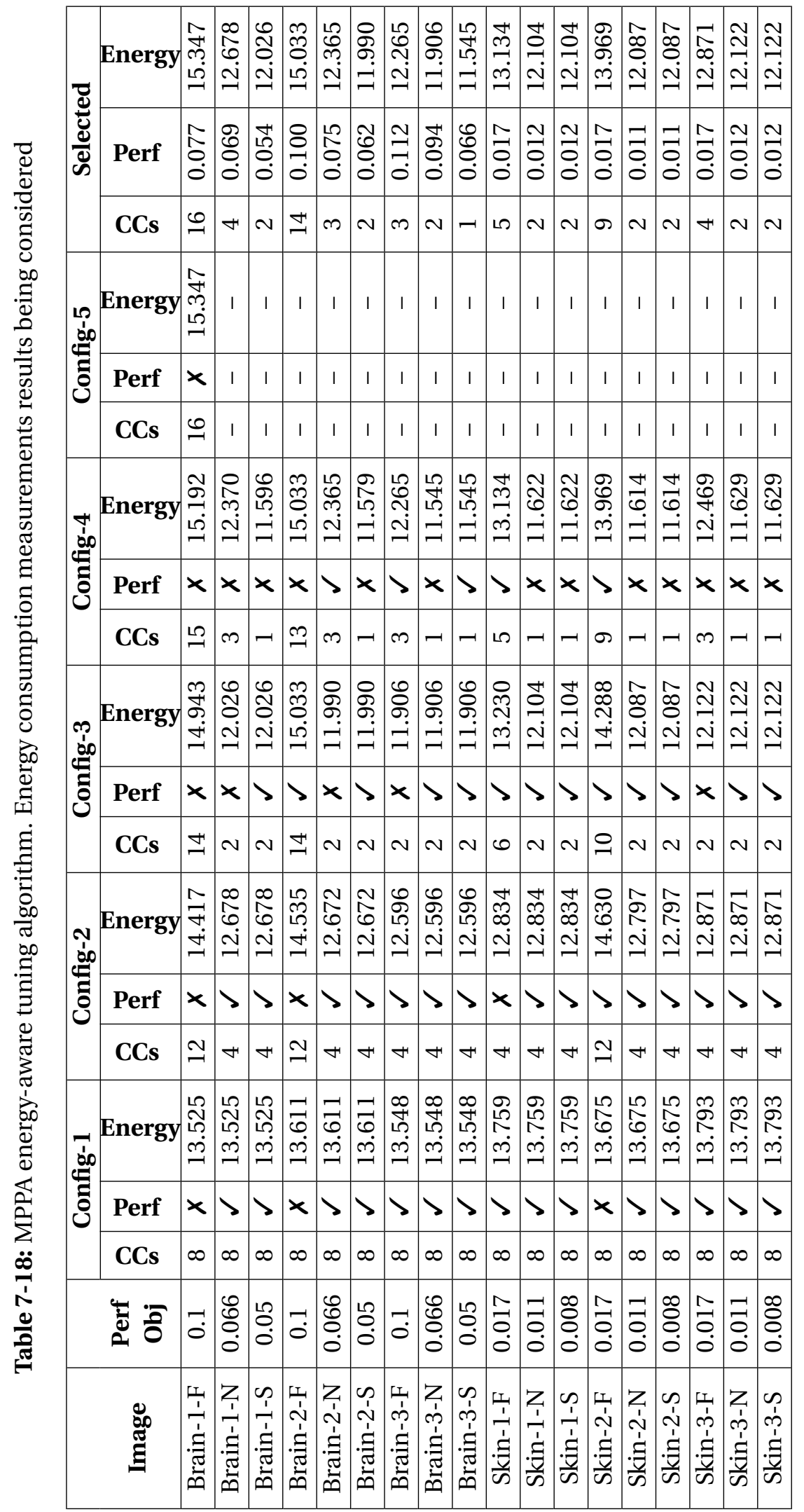




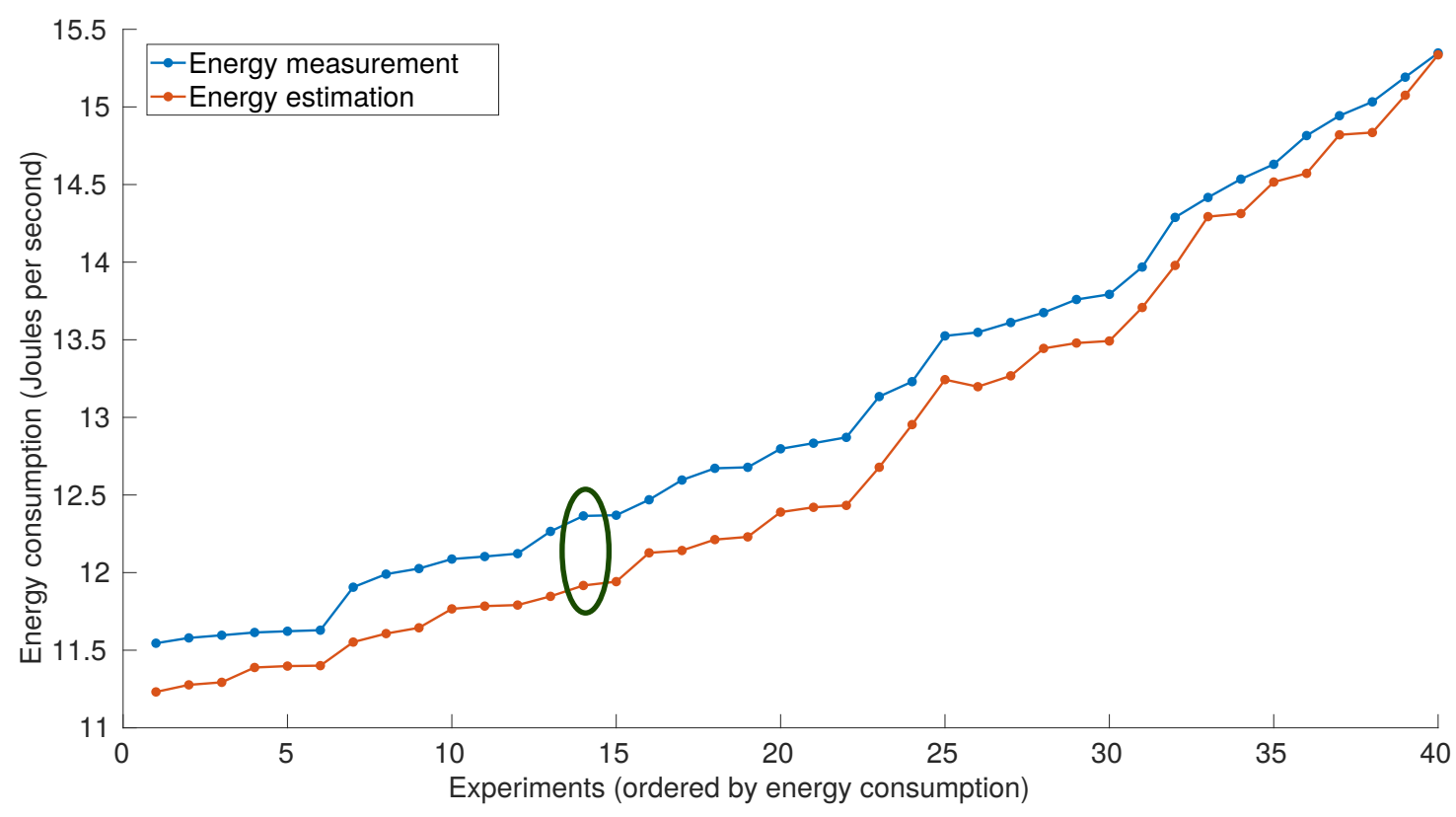

Figure 7-26: MPPA energy consumption model evaluation running the HSI cancer detection algorithm. Measurement vs estimation. Selected maximum error of $3.62 \%$

Table 7-19: Performance (images per second), power (W), and power savings (\%) of the HSI processing chain for the validation experiments. Implementation by hand vs runtime energyaware optimization loop. An experiment is valid if it reaches the performance objective

\begin{tabular}{|c|c|c|c|c|c|c|c|}
\hline \multirow[b]{2}{*}{ Experiment } & \multicolumn{2}{|c|}{$\begin{array}{c}\text { Implementation } \\
\text { by hand }\end{array}$} & \multicolumn{2}{|c|}{$\begin{array}{c}\text { Runtime } \\
\text { energy-awareness }\end{array}$} & \multicolumn{3}{|c|}{ Evaluation } \\
\hline & Perf & Power & Perf & Power & $\begin{array}{c}\text { Perf } \\
\text { objective }\end{array}$ & Valid & $\begin{array}{l}\text { Power } \\
\text { savings }\end{array}$ \\
\hline Brain-1-F & \multirow{3}{*}{0.357} & \multirow{3}{*}{16.18} & 0.077 & 15.347 & 0.1 & $x$ & - \\
\hline Brain-1-N & & & 0.069 & 12.678 & 0.066 & $\checkmark$ & 21.641 \\
\hline Brain-1-S & & & 0.054 & 12.026 & 0.05 & $\checkmark$ & 25.673 \\
\hline Brain-2-F & \multirow{3}{*}{0.392} & \multirow{3}{*}{15.96} & 0.100 & 15.033 & 0.1 & $\checkmark$ & 5.810 \\
\hline Brain-2-N & & & 0.075 & 12.365 & 0.066 & $\checkmark$ & 22.524 \\
\hline Brain-2-S & & & 0.062 & 11.990 & 0.05 & $\checkmark$ & 24.874 \\
\hline Brain-3-F & \multirow{3}{*}{0.567} & \multirow{3}{*}{15.24} & 0.112 & 12.265 & 0.1 & $\checkmark$ & 19.518 \\
\hline Brain-3-N & & & 0.094 & 11.906 & 0.066 & $\checkmark$ & 21.877 \\
\hline Brain-3-S & & & 0.066 & 11.545 & 0.05 & $\checkmark$ & 24.245 \\
\hline Skin-1-F & \multirow{3}{*}{0.090} & \multirow{3}{*}{17.28} & 0.017 & 13.134 & 0.017 & $\checkmark$ & 23.992 \\
\hline Skin-1-N & & & 0.012 & 12.104 & 0.011 & $\sqrt{ }$ & 29.956 \\
\hline Skin-1-S & & & 0.012 & 12.104 & 0.008 & $\checkmark$ & 29.956 \\
\hline Skin-2-F & \multirow{3}{*}{0.090} & \multirow{3}{*}{17.32} & 0.017 & 13.969 & 0.017 & $\sqrt{ }$ & 19.350 \\
\hline Skin-2-N & & & 0.011 & 12.087 & 0.011 & $\checkmark$ & 30.212 \\
\hline Skin-2-S & & & 0.011 & 12.087 & 0.008 & $\sqrt{ }$ & 30.212 \\
\hline Skin-3-F & \multirow{3}{*}{0.090} & \multirow{3}{*}{17.35} & 0.017 & 12.871 & 0.017 & $\checkmark$ & 25.814 \\
\hline Skin-3-N & & & 0.012 & 12.122 & 0.011 & $\sqrt{ }$ & 30.132 \\
\hline Skin-3-S & & & 0.012 & 12.122 & 0.008 & $\checkmark$ & 30.132 \\
\hline
\end{tabular}


The last analysis focuses on graphically evaluating the power/energy savings obtained when simulating the runtime optimization loop on the MPPA. These results are displayed in Figure 7-27, where the power/energy consumption -in W- of every implementation is represented using bars and grouped by the hyperspectral image being processed (horizontal axis). Additionally, the power/energy savings -in \%themselves are plotted using full, dashed and pointed lines for Fast, Normal and Slow working modes, respectively. As can be seen, once the simulated energy-aware optimization loop optimizes the implementation in terms of energy consumption, power/energy savings from $5.810 \%$ (in Brain-2-F) to $30.212 \%$ (in Skin-2-N and Skin$2-S$ ) are obtained using a real use-case application.

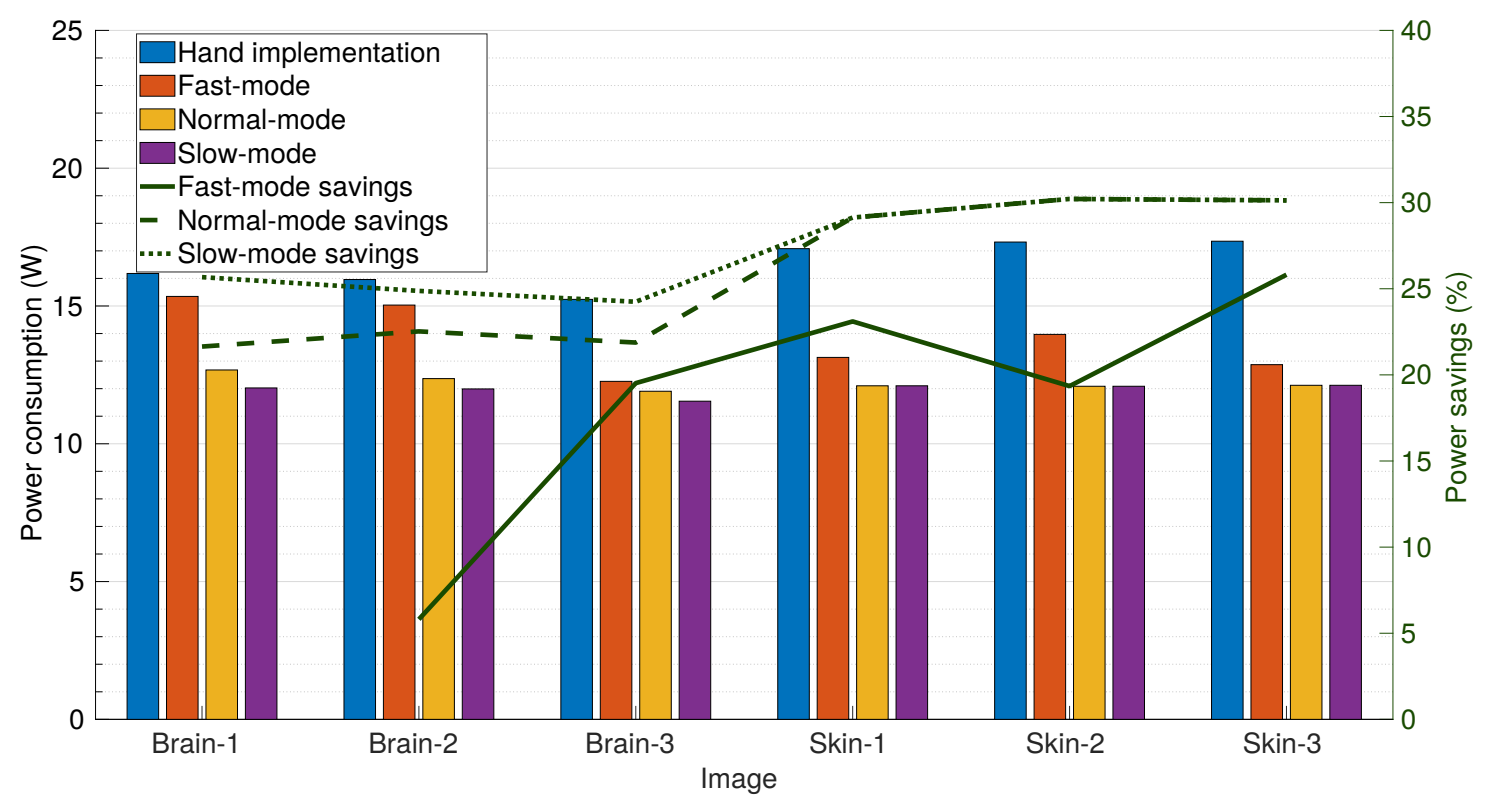

Figure 7-27: Runtime energy-aware optimization loop results for the MPPA architecture. Analysis of the energy savings obtained for the different working modes

During this section, it has been demonstrated that the MPPA energy consumption model provides highly accurate estimations while running a real-life application. Hence, its use within a runtime energy-aware optimization loop, as the one shown in Subsection 7.4.3, has been proven to be feasible. Specifically, simulating this optimization loop on the MPPA energy savings up to $30.212 \%$ have been reached. As a final conclusion, it is easily foreseen that, combining the results of Subsection 7.4.3 and the ones shown in this section, the energy-aware optimization loop embedded within SPiDER could provide promising results when running on the MPPA architecture. 


\subsection{Discussion}

Along this chapter, the HSI cancer detection processing chain has been implemented and evaluated using different methodologies. First, it has been implemented by hand on the MPPA, exploiting as much as possible the parallelism and optimizing the latency. Then, a dataflow version of the application has been developed in PREESM and optimized 1) in terms of latency and 2) using the energy-aware optimization loop presented in Chapter 6. Finally, a runtime dynamic version of the algorithm has been evaluated using SPiDER runtime manager and optimized, again, with and without considering energy consumption.

Regarding the implementation done by hand, depending on the spatial resolution of the hyperspectral image, speedups from $49.696 \times$ to $112.129 \times$ have been achieved when comparing the sequential execution with the one using the 256 MPPA PEs. Additionally, although the maximum performance is, in average, 6 times higher than the one obtained with the dataflow implementation, it required a deep knowledge about both the HSI cancer detection algorithm and the MPPA many-core architecture.

In the case of PREESM implementation, when the optimization loop is used to minimize the latency, thanks to the timing data retrieved via PAPIFY, speedups from $1.1 \times$ to $1.4 \times$ have been achieved. Likewise, when energy-awareness is included within the system requirements, power/energy savings up to $30 \%$ have been automatically obtained thanks to the design time energy-aware optimization loop.

Finally, the runtime optimization loop has been validated by means of a twostep methodology. First, using a multi-core architecture gathering 16 x86 cores, the application has been optimized taking decisions on-the-fly. In the case of the latencyoptimization, speedups from $1.019 \times$ to $1.338 \times$ are obtained. Then, using a simulated energy consumption model, power/energy savings up to $25.279 \%$ are reached. After this step, the energy-aware optimization loop is simulated on the MPPA, where the energy consumption model developed in Chapter 5 is used to estimate this figure of merit. As a result, power/energy savings up to $30.212 \%$ have been proven achievable.

Thanks to the dataflow approach, the system can be automatically deployed over the MPPA/resources considering not only latency/timing information, but also taking into consideration energy consumption so as to perform automatic optimizations of this figure of merit. In this case, the required level of expertise associated to the target architecture is lower than when implementing the application by hand. 


\section{CONCLUSIONS}

This chapter concludes the manuscript by summarizing the conclusions and contributions of the proposed solutions. Additionally, a set of future research lines associated with this work are also explained.

In first place, the main conclusions that can be extracted from the analysis of the work proposed in this $\mathrm{PhD}$ are presented in Section 8.1. After this, Section 8.2 outlines the main contributions of this thesis, indicating the sections associated to each of them. Then, Section 8.3 explains the academic results carried out along this $\mathrm{PhD}$, including scientific publications, research projects, collaborations, grants and awards obtained. Finally, Section 8.4 introduces a set of future research lines that are proposed to enhance and take advantage of the work developed during this thesis.

\subsection{Conclusions}

The work proposed in this $\mathrm{PhD}$ has been motivated by the growing complexity of both applications and architectures, together with the tightening of functional and non-functional system requirements. Specifically, the reference use-case, which is an $\mathrm{HSI}$ cancer detection processing chain that distinguishes tumor boundaries, has been applied to two different application fields: neurosurgical and dermatological. The former, where human brain cancer is delineated, presents tight performance requirements and loose energy consumption constraints. The latter, which detects skin tumor boundaries, is characterized by loose performance requirements and tight energy consumption constraints.

To tackle this problematic, as introduced in Section 1.2, the main objective of this work [OBJ-P] has been to define a methodology and its associated toolchain to optimize latency and energy consumption of dataflow applications running on multi/many-core architectures at both design time and runtime. Therefore, a Y-chart dataflow-based energy-aware optimization loop has been proposed to be applicable at both design time and runtime. On the one hand, the loop has been included within PREESM dataflow-based development framework where, after profiling the application in terms of execution time and energy consumption per actor, energy-aware DSE is used to minimize the energy consumption of the generated application at design time. 
On the other hand, the loop has been embedded within SPiDER runtime resource manager to perform on-the-fly energy-aware optimizations based on information directly extracted during the application execution.

Prior to applying any automatic optimization [OBJ-S1], the reference application has been manually implemented on the MPPA many-core architecture to deeply understand the details associated to the use-case (Subsection 7.2.1). In this study, the main limitation has been linked to the reduced amount of available memory on the MPPA architecture, which involves exploiting the intrinsic parallelism of the HSI cancer detection processing chain. During the implementation, the main objective has been to minimize the latency, while no energy constraints have been considered. As a result, the required time to process the hyperspectral images has been reduced to up to 10 s, achieving speedups from $49.696 \times$ to $112.129 \times$ when the processing is distributed among all the available platform $\mathrm{PE}$ s Subsection 7.2.2.

Then, a dataflow version of the application has been developed to be automatically deployed on the architecture [OBJ-S1]. As shown in Subsection 7.3.3, the performance that can be reached with this approach is, in average, 6 times lower than the one achievable with the implementation done by hand. Nevertheless, using the dataflow approach, the energy consumption can be automatically reduced by means of the energy-aware optimization loop proposed in this $\mathrm{PhD}$.

In the case of applying the energy-aware optimization loop at design time, although the time required by PREESM to generate the system is 3-4 times larger when considering energy-awareness, when compared to the implementation carried out by hand, power/energy savings from $0.05 \%$ to $30.21 \%$ are reached (Subsection 7.3.4).

Regarding the runtime energy-aware optimization loop, although its validation has been carried out in two steps, the benefits obtained in this case are even higher. First, considering a simulated energy consumption model of a 16-core x86 architecture, on-the-fly power/energy consumption reductions up to $25.279 \%$ are achieved (Subsection 7.4.3). Then, simulating the loop on the MPPA platform, where the real energy consumption model of the architecture is used, power/energy savings from $5.81 \%$ to $30.21 \%$ are reached, when compared to the implementation done by hand (Subsection 7.4.4). In this case, it should be highlighted that a system provided with self-awareness and reactiveness to dynamic behaviors -changes on the image resolution or variations of the performance objective- has been built.

The definition of the energy-aware optimization loop has been possible thanks to the fulfilling of 3 secondary objectives, which are linked to the blocks composing the loop itself: monitoring infrastructure [OBJ-S2] (Chapter 4), KPI estimation [OBJ-S3] Chapter 5) and energy-awareness [OBJ-S4] Chapter 6.

The monitoring infrastructure [OBJ-S2] has been defined by means of a tool called PAPIFY. This infrastructure has been developed to transparently profile applications based on hardware performance information and following a Y-chart dataflowbased approach. That is, the characterization is performed considering the actor being executed and the $\mathrm{PE}$ executing it. This monitoring infrastructure has been 
validated through two studies. First, timing information has been retrieved with PAPIFY to optimize the dataflow version of the HSI cancer detection application in terms of latency. In this study, speedups up to $1.4 \times$ Subsection 7.3.3 and 1.338× Subsection 7.4.2) have been achieved for the MPPA at design time and for the x86 architecture at runtime, respectively. Then, PMC data have been retrieved in both cases to estimate energy consumption as part of the energy-aware optimization loop.

In this $\mathrm{PhD}$, the KPI that has been optimized is the energy consumption of the system. To do so, the energy consumption modeling approach proposed in this work [OBJ-S3] is based on hardware utilization information that can be retrieved at runtime. In this regard, the MPPA energy consumption has been modeled focusing on developing an application-agnostic model. Specifically, two validations have been carried out considering applications that have not been included when building the model itself: using a GeMM application, an average accuracy of $95 \%$ is achieved (Subsection 5.7.2); and using the HSI cancer detection processing chain, the average accuracy is 96\% (Subsection 7.3.4 and Subsection 7.4.4). Consequently, the application-agnostic model of the MPPA has been successfully developed.

Finally, an energy-aware decision making mechanism has been defined as part of the optimization loop [OBJ-S4]. The so-called energy-awareness tuning algorithm surrounds a latency-based mapping/scheduling algorithm with a searching protocol that looks for the system configuration with the least energy consumption, while fulfilling a user-defined performance objective. To be precise, this mechanism has been used to enhance the DSE capabilities of PREESM and to perform on-the-fly energy optimizations in SPiDER.

\subsection{Contributions}

Once the main conclusions of this $\mathrm{PhD}$ have been analyzed and linked them with the objectives of the thesis, in this section, the original contributions associated to this work are summarized. First, the contributions associated to the use-case that motivated the development of the design methodology proposed in this $\mathrm{PhD}$ are gathered in Subsection 8.2.1. Then, the independent blocks of the loops are analyzed to highlight their individual contributions in the field of 1) monitoring (Subsection 8.2.2), 2) energy modeling (Subsection 8.2.3) and 3) energy-awareness (Subsection 8.2.4). Finally, Subsection 8.2.5 collects the contributions associated to the complete energyaware optimization loop, considering its integration with PREESM (design time) and SPiDER (runtime) and its validation using the HSI cancer detection processing chain. 


\subsubsection{HSI cancer detection}

In this $\mathrm{PhD}$, cancer detection based on HSI technology has been considered the main motivation to develop a new design methodology to ease the implementation of highly demanding applications over complex architectures, as explained in Chapter 3 . Consequently, this use-case has been used to validate the proposed methodology in Chapter 7. In this regard, this $\mathrm{PhD}$ presents the following original contributions in the context of HSI applied to cancer detection:

- MPPA implementation by hand done in the context of HELICoiD European project. An implementation on the MPPA many-core architecture has been developed aiming at delineating human brain cancer boundaries as fast as possible. This version is thoroughly detailed in Section 7.2 and used as a reference to evaluate the benefits of the proposed design methodology.

- Dataflow static version developed using PREESM framework and its associated PiSDF MoC, This implementation has been detailed in Subsection 7.3.2 and evaluated in Subsection 7.3.3 and Subsection 7.3.4. Although the performance of PREESM deployment onto the MPPA is around 6 times lower than the implementation done by hand, it presents the following advantages:

- It has been parameterized, so its parallelization degree can be defined using PREESM framework.

- Its portability has increased, as it can be deployed onto the different architectures supported by the framework.

- Memory constraints have been considered, and tensor-based processing has been included.

- Its modular implementation allows developers to change the algorithms running inside each block as far as inputs and outputs are not modified.

- Dataflow dynamic version implemented targeting SPiDER runtime manager. In this implementation, as explained in Subsection 7.4.1 dynamism is included to support the image resolutions and the performance objective changing at runtime. This version is the one used in Section 7.4.

\subsubsection{Monitoring}

Regarding application monitoring, the original contribution of this thesis is linked to the development of PAPIFY monitoring infrastructure, explained in Chapter 4. Additionally, this monitoring infrastructure has been validated while profiling the dataflow version of the HSI cancer detection processing chain employed in Section 7.3 and Section 7.4. The main characteristics of this monitoring infrastructure are: 
- Transparent access to PMC information: since PAPIFY is built on top of PAPI library, it can transparently access to each PAPI component to retrieve specific PMC information depending on the resource being profiled.

- Versatile monitoring support: in this sense, with PAPIFY, each part of the application can be instrumented with a different monitoring configuration. As a result, for each part of the application, developers decide if they want to monitor only timing (for latency optimization purposes), PMCs (for energy optimization purposes, for example) or both data sources (for multi-objective optimization).

- Runtime support: with PAPIFY, each processing element existing within the target architecture is linked to one specific PAPI component, thus, only the available $\mathrm{PMC} s$ are accessible from each processing resource. As a result, even if the application is redistributed at runtime, PAPIFY runtime selects the desired monitoring configuration for each part of the application considering the PMCs that are available on the resource executing it.

- Heterogeneous architecture support: PAPIFY allows the monitoring of heterogeneous architectures where, for example, CPUs collaborate with an FPGA. In this situation, a single API is used and, thus, PAPIFY provides transparent and homogeneous access to PMC information.

Summing up, this monitoring infrastructure provides transparent and uniform access to PMC information following a Y-chart dataflow-based strategy. This means that, as a result, PAPIFY supports the characterization of 1) applications redistributed (or not) at runtime 2) with versatile monitoring configurations for each part of the application running on 3) heterogeneous architectures where computational resources of different nature collaborate -e.g., CPUs and FPGA

Additionally, one of the instrumental contributions of this $\mathrm{PhD}$ is the support of PAPI library on the MPPA to provide transparent access to hardware performance information. This work is detailed in Section 5.3, first used to develop the energy consumption model Section 5.6) and then again to profile the HSI cancer detection processing chain in Chapter 7 .

As highlighted in Subsection 7.3.3 and Subsection 7.4.2, the use of PAPIFY profiling to update timing information -without considering any energy constraintsallows PREESM and SPiDER to enhance their already existing latency-based mapping/schedulings. As a result, speedups up to $1.4 \times$ and $1.338 \times$ are achieved when using design time DSE (PREESM and the MPPA) and runtime optimizations SPiDER and an x86 architecture), respectively. 


\subsubsection{Energy modeling}

In this $\mathrm{PhD}$, a new energy modeling approach -based on runtime hardware performance information- has been proposed in Chapter 5 and used to build an energy consumption model of the MPPA-256-N architecture developed by Kalray. Then, this energy consumption model has been employed to estimate the energy consumption of different application deployments onto the MPPA platform in Chapter 7. This modeling methodology, which is considered one of the original contributions of this $\mathrm{PhD}$, is characterized by the following features:

- Modularity: the energy consumption of the platform is divided into 3 parts: resource-active, communication and computation:

- Resource-active contribution: linked to the number of active resources.

- Communication contribution: associated to the data tokens transmitted among platform resources.

- Computation contribution: related to the execution of tasks by the computational resources.

- Runtime information: the modeling technique is based on information that can be retrieved at runtime, so as to perform on-the-fly energy consumption estimations if needed. For each model module the information is:

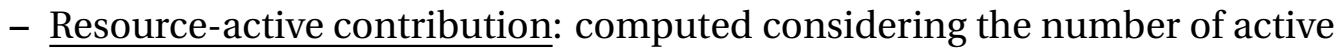
resources and its individual contributions.

- Communication contribution: modeled considering the size of the token transmitted, the source and the destination of the communication, which may refer to read or write in different memory types SMEM or DDR in the case of the MPPA, respectively.

- Computation contribution: estimated using PMC event information, which reflects the hardware utilization during a specific time interval.

- Simplicity: with the objective of being able to use the energy consumption model at runtime, linear equations are considered when building the model.

This modeling approach has been applied onto the MPPA architecture. As a result, during the validation of the model -Subsection 5.7.2-, an average relative error below $5 \%$ has been achieved using GeMM application. Additional, in the case of estimating the energy consumption of a real application like the HSI cancer detection algorithm, the maximum relative error in this case is only $3.7 \%$ and $3.62 \%$ when predicting the energy consumption at design time -Subsection 7.3.4 or estimated at runtime Subsection 7.4.4-, respectively. 


\subsubsection{Energy-awareness}

Concerning energy-awareness research field, in Section 6.2, an iterative procedure, called energy-aware tuning algorithm, has been proposed as an original contribution of this PhD. This searching algorithm presents the following characteristics:

- Objective: the mechanism seeks the system configuration that minimizes the energy consumption, while reaching a user-defined performance objective.

- System configurations tested: a set of system configurations, defined by the number of active $\mathrm{PE} s$ used to run the application, are considered as candidates.

- System generation: a latency-based mapping/scheduling algorithm is used to generate the applications based on the different system configurations. This algorithm is considered a black box during the procedure.

- Evaluation: each system configuration is characterized in terms of performance and energy consumption.

- Energy-aware decision making: the tested configurations are selected considering the performance obtained when evaluating each candidate configuration. If this value does not reach the performance objective, the number of active PEs is increased, and vice-versa.

- Solution: once the searching finishes, the optimum configuration is selected among the system configurations candidates.

Since the approach is generic, it can be applied in different contexts and adapted by different frameworks, e.g., to perform DSE at design time (PREESM-Section 6.3 or to perform on-the-fly runtime optimizations (SPiDER-Section 6.4-).

\subsubsection{Iterative energy-aware optimization loop}

To define the methodology proposed in this $\mathrm{PhD}$, the Y-chart dataflow-based energyaware optimization loop has been built combining the 3 individual blocks explained before, whose behavior is summarized in Figure 6-2, This approach has been particularized to optimize energy consumption, but, due to its modular nature, it can be easily generalizable. The functionality of the 4 blocks composing the loop are:

- Monitoring: in this step, the application is profiled and performance information is extracted from real executions.

- KPI estimation: this block analyzes the information of the monitoring step and translates it into relevant information, i.e., KPIs. In this particular case, the KPI is the energy consumption. 
- Multi-objective decision making: this mechanism evaluates the current system configuration and decides whether a change is required. In the energy-aware optimization loop, the decision is made considering whether a performance objective is reached or not.

- Application deployment: considering the outcome of the decision making block, this step adapts the system implementation accordingly.

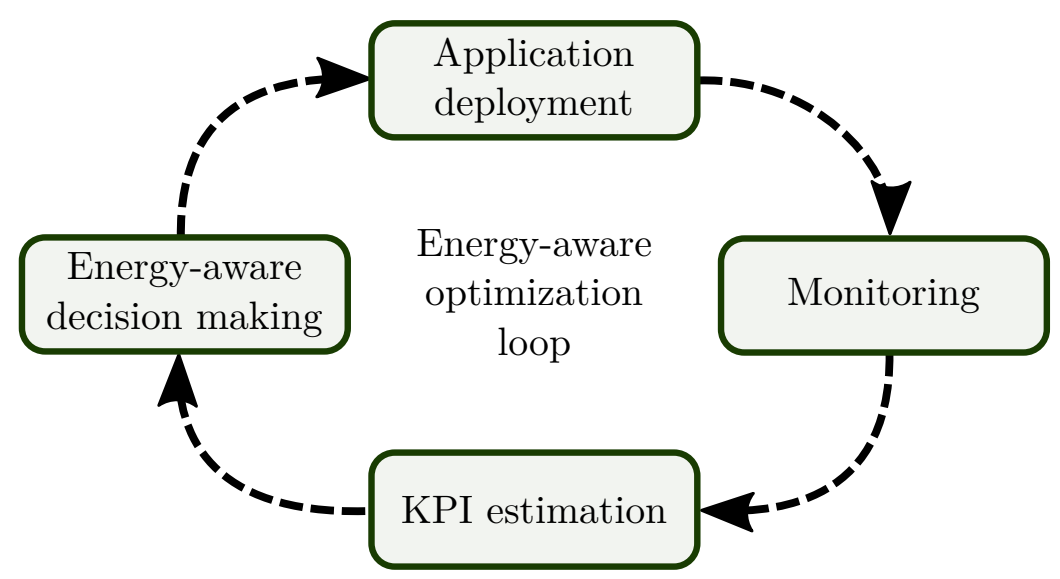

Figure 6-2 Energy-aware optimization loop (repeated from page 107

In this thesis, two energy-aware optimization loops have been presented as original contributions of this PhD: one targeting DSE enhancement at design time and the other aiming at performing on-the-fly runtime optimizations.

- Design time DSE enhancement in PREESM as explained in Section 6.3, an enhanced version of DSE in PREESM has been included to consider not only latency-based optimization, but also to minimize the energy consumption of the deployed system. This methodology presents the following characteristics:

- Monitoring: timing and PMCinformation are extracted using PAPIFY during real system executions. After this, the information is included within PREESM scenario for it to be available during DSE.

- KPI estimation: the energy consumption model is included within PREESM scenario. By doing so, PMC information extracted during the monitoring step can be directly translated into energy consumption of each actor when being executed by each type of PE

- Energy-aware decision making: during the DSE procedure, as explained in Subsection 8.2.4, the energy-aware tuning algorithm searches the system configuration that fulfills a performance objective, while presenting the lowest energy consumption. To do so, the latency-based mapping/scheduling algorithm is used as a black box and both performance and energy consumption values are predicted. 
- Application deployment: the standard workflow of PREESM is used and, thus, the application is generated as usual, but considerıng the decision of the energy-aware decision making procedure.

In this regard, the methodology has been validated using the HSI cancer detection algorithm applied in both neurosurgical and dermatological fields. This validation is detailed in Subsection 7.3.4 and presents the following results:

- The DSE generation time when energy-awareness is included is 3-4 times the one required when only latency is considered.

- Power/energy consumption savings from $0.05 \%$ to $30.21 \%$ are obtained when compared to the implementation done by hand.

- Runtime optimization loop in SPiDER as explained in Section 6.4, an energyaware optimization loop has been embedded within SPiDER GRT In this case, different configurations are evaluated in each graph iteration until the optimal solution is reached. The main features of this self-adaptation loop are:

- Monitoring: timing and PMCinformation are extracted using PAPIFY during real system executions. Then, these data are fed to SPiDER GRT, keeping the manager informed of the status and characterization of the system.

- KPI estimation: once the information is received in the GRT actor information when executed on each type of processing element is updated. Specifically, timing information is directly linked to each actor, while energy consumption is estimated based on the PMC information.

- Energy-aware decision making: in each iteration of the application, a different number of enabled/disabled PEs is considered when mapping/scheduling the application using a standard latency-based algorithm, which is considered a black-box. After some iterations, an optimal solution is found and kept during the rest of the system execution. Afterwards, the system performance is continuously monitored and, if the objective is not reached during a number of iterations, a safeguard is triggered and the number of active processing elements is increased.

- Application deployment: the standard latency-based mapping/scheduling algorithm of $[$ SPiDER is used to generate the application, but the active PEs, used as input of the algorithm, are modified according to the energy-aware decision making outcomes.

The self-adaptation loop has been validated using the HSI cancer detection algorithm applied in neurosurgical and dermatological fields. This validation is detailed in Subsection 7.4.3 and the potential benefits that could be obtained on the MPPA architecture are shown in Subsection 7.4.4, These results are:

- The self-adaptation loop selects the system configuration with the lowest energy consumption that reaches the performance objective. 
- The system reacts to dynamic changes in the application parameters and adapts the selected system configuration accordingly.

- Using an x86 architecture, power/energy consumption savings up to $25.279 \%$ are achieved. These results have been obtained using a simulation of the energy consumption model for this architecture.

- Simulating the iterative energy-awareness loop on the MPPA platform, power/energy consumption savings from $5.810 \%$ to $30.212 \%$ are reached when compared to the implementation done by hand.

\subsection{Impact of the thesis}

This section summarizes the academic results associated to the development of this PhD. These academic results are organized in 1) publications, 2) related research projects, 3) collaborations with other institutions, 4) open-access products, 5) grants received and 6) awards obtained.

\subsubsection{Publications and dissemination}

During the development of this $\mathrm{PhD}$, several publications have been carried out showing some of the contributions proposed in this work. Additionally, scientific dissemination has been achieved by means of posters, demonstrators and tutorials.

\section{Journal publications}

The author of this thesis has authored/co-authored 11 journal articles related with the work presented in this manuscript:

[Lazcano'19b] R. Lazcano, D. Madroñal, G. Florimbi, J. Sancho, S. Sanchez, R. Leon, H. Fabelo, S. Ortega, E. Torti, R. Salvador, et al., "Parallel Implementations Assessment of a Spatial-Spectral Classifier for Hyperspectral Clinical Applications", in IEEE Access, vol. 7, pp. 152316-152 333, 2019. Q1

This paper presents a study where different hardware accelerators, GPUs and the MPPA, are compared in terms of performance and energy consumption when running the supervised classification block of HELICoiD HSI cancer detection processing chain. The MPPA implementation presented in this paper is the one used in Section 7.2. 
[Madronal'19a] D. Madronal, F. Arrestier, J. Sancho, A. Morvan, R. Lazcano, K. Desnos, R. Salvador, D. Menard, E. Juarez, C. Sanz, "PAPIFY: Automatic Instrumentation and Monitoring of Dynamic Dataflow Applications Based on PAPI", in IEEE Access, vol. 7, pp. $111801-111812$, 2019. Q1

In this paper, PAPIFY monitoring infrastructure is presented together with its integration within PREESM and SPiDER, PAPIFY monitoring infrastructure is thoroughly explained in Chapter 4 and used to characterize the HSI dataflow application in Section 7.3 and Section 7.4.

[Fabelo'19b H. Fabelo, S. Ortega, A. Szolna, D. Bulters, J. F. Piñeiro, S. Kabwama, A. JO'Shanahan, H. Bulstrode, S. Bisshopp, B. R. Kiran, et al., "In-Vivo Hyperspectral Human Brain Image Database for Brain Cancer Detection”, in IEEE Access, vol. 7, pp. 39 098-39 116, 2019. Q1

This paper presents the databased generated in HELICoiD project, composed of human brain hyperspectral images. The Brain application field images used in Chapter 7 have been extracted from this database.

[Florimbi'18] G. Florimbi, H. Fabelo, E. Torti, R. Lazcano, D. Madroñal, S. Ortega, R. Salvador, F. Leporati, G. Danese, A. Báez-Quevedo, et al., "Accelerating the Knearest neighbors filtering algorithm to optimize the real-time classification of human brain tumor in hyperspectral images", in Sensors, vol. 18, no. 7, p. 2314, 2018. Q1

In this paper, a study of the $\mathrm{KNN}$ algorithm, which is included within the supervised classification block of the HELICoiD HSI processing chain, on a GPU is exposed. Specifically, an implementation that optimizes and parallelizes the algorithm is sought.

[Martel'18] E. Martel, R. Lazcano, J. López, D. Madroñal, R. Salvador, S. López, E. Juarez, R. Guerra, C. Sanz, R. Sarmiento, "Implementation of the principal component analysis onto high-performance computer facilities for hyperspectral dimensionality reduction: Results and comparisons", in Remote Sensing, vol. 10, no. 6, p. 864, 2018. Q1

This paper introduces a comparative study of the PCA algorithm, which is included within the supervised classification block of the HELICoiD HSI processing chain, onto a GPU and the MPPA architecture using hyperspectral images belonging to the remote sensing field.

Lazcano’19a R. Lazcano, D. Madroñal, H. Fabelo, S. Ortega, R. Salvador, G. Callico, E. Juarez, C. Sanz, "Adaptation of an iterative PCA to a manycore architecture for hyperspectral image processing", in Journal of Signal Processing Systems, vol. 91, no. 7, pp. 759-771, 2019. Q4

This paper evaluates the implementation of an iterative version of the PCA algorithm, called Non-Linear Iterative Partial Least Squares (NIPALS), on the MPPA architecture. 
[Fabelo'18b H. Fabelo, S. Ortega, D. Ravi, B. R. Kiran, C. Sosa, D. Bulters, G. M. Callicó, H. Bulstrode, A. Szolna, J. F. Piñeiro, et al., "Spatio-spectral classification of hyperspectral images for brain cancer detection during surgical operations", in PloS one, vol. 13, no. 3, p. e0193 721, 2018. Q2

In this paper the HSI processing chain used in HELICoiD is presented. The supervised classification block of this processing chain is considered the usecase of this PhD. It is detailed in Subsection 3.1.1 and used in Chapter 7 to validate the proposed contributions of this work.

[Fabelo'18a] H. Fabelo, S. Ortega, R. Lazcano, D. Madroñal, G. M Callicó, E. Juárez, R. Salvador, D. Bulters, H. Bulstrode, A. Szolna, et al., "An intraoperative visualization system using hyperspectral imaging to aid in brain tumor delineation", in Sensors, vol. 18, no. 2, p. 430, 2018. Q1

This paper details the demonstrator developed as an output of HELICoiD project, which has been used to help surgeons while delineating the boundaries of a human brain cancer in the operating theater. Thanks to this demonstrator, the database containing the Brain hyperspectral images used in Chapter 7 to validate the proposed contributions of this work has been built.

[Madroñal'17b] D. Madroñal, R. Lazcano, R. Salvador, H. Fabelo, S. Ortega, G. M. Callicó, E. Juarez, C. Sanz, "SVM-based real-time hyperspectral image classifier on a manycore architecture", in Journal of Systems Architecture, vol. 80, pp. 30-40, 2017. Q3

This paper presents a study of the design space implementations of the SVM classifier on the MPPA architecture. The SVM implementation presented in Subsection 7.2.1 is an improved version of the one presented in this paper.

[Lazcano'17c] R. Lazcano, D. Madroñal, R. Salvador, K. Desnos, M. Pelcat, R. Guerra, H. Fabelo, S. Ortega, S. López, G. M. Callicó, et al., "Porting a PCA-based hyperspectral image dimensionality reduction algorithm for brain cancer detection on a manycore architecture", in Journal of Systems Architecture, vol. 77, pp. 101-111, 2017. Q3

In this paper, the parallelism of the $\mathrm{PCA}$ algorithm, divided in stages, is analyzed using the MPPA architecture in the context of HELICoiD project. This parallelism, with a different implementation, is the one used in Subsection 7.2.1.

|Ortega'17| S. Ortega, H. Fabelo, R. Camacho, M. Plaza, G. Callico, R. Lazcano, D. Madroñal, R. Salvador, E. Juárez, R. Sarmiento, "P03. 18 Detection of human brain cancer in pathological slides using hyperspectral images", in Neurooncology, vol. 19, no. Suppl 3, p. iii37, 2017. Q1

In this paper, preliminary results of the research performed within HELICoiD project to delineate human brain tumor boundaries are presented. 


\section{Conference publications}

Concerning the publications in international conferences, the following contributions related with the work presented during this $\mathrm{PhD}$ have been authored/co-authored during the development of this thesis:

[Lazcano'20] R. Lazcano, D. Madroñal, E. Juarez, P. Clauss, "Runtime multi-versioning and specialization inside a memoized speculative loop optimizer", in Proceedings of the 29th International Conference on Compiler Construction, pp. 96-107, 2020.

This paper presents a set of modifications carried out within Automatic Speculative Polyhedral Loop Optimizer Apollo so as to enhance its optimization capabilities through a runtime multiversioning system including memoization and code specialization. This work is included within CERBERO project.

|Aragón’19] J. S. Aragón, S. S. Ramírez, R. L. López, D. M. Quintín, R. S. Perea, E. J. Martínez, C. S. Álvaro, "Characterizing Hyperspectral Data Layouts: Performance and Energy Efficiency in Embedded GPUs for PCA-based Dimensionality Reduction", in 2019 XXXIV Conference on Design of Circuits and Integrated Systems (DCIS), pp. 1-6, IEEE, 2019.

This paper analyzes the performance and energy efficiency of an embedded GPU when using dimensionality reduction algorithms, such as PCA. with different data layouts for hyperspectral images.

Fanni'19 T. Fanni, D. Madronal, C. Rubattu, C. Sau, F. Palumbo, E. Juarez, M. Pelcat, C. Sanz, L. Raffo, "Run-time Performance Monitoring of Heterogenous Hw/Sw Platforms Using PAPI", in FSP Workshop 2019; Sixth International Workshop on FPGAs for Software Programmers, pp. 1-10, VDE, 2019.

In this paper, the combination of PAPIFY and Multi-Dataflow Composer (MDC) tools is presented. As part of CERBERO project, this combination of tools provides developers with a toolchain capable of implementing Coarse-Grain Virtual Reconfigurable Circuits instrumented with custom monitors targeting a unified approach for monitoring heterogeneous HW/SW platforms. This work applies PAPIFY monitoring infrastructure explained in Chapter 4 in a heterogeneous system.

[Palumbo'19b $\mid$ F. Palumbo, T. Fanni, C. Sau, A. Rodríguez, D. Madroñal, K. Desnos, A. Morvan, M. Pelcat, C. Rubattu, R. Lazcano, et al., "Hardware/Software Selfadaptation in CPS: The CERBERO Project Approach", in International Conference on Embedded Computer Systems, pp. 416-428, Springer, 2019.

In this paper, an overview of CERBERO project is provided, focusing on the proposed hardware/software self-adaptation runtime loop. The monitoring infrastructure used in this paper is PAPIFY - presented in Chapter 4-. Additionally, the CERBERO self-adaptation runtime loop is the one that inspires the energyaware optimization loop proposed in this $\mathrm{PhD}$-explained in Chapter 6 . 
[Suriano'18] L. Suriano, D. Madroñal, A. Rodríguez, E. Juárez, C. Sanz, E. de la Torre, "A unified hardware/software monitoring method for reconfigurable computing architectures using PAPI", in 2018 13th International Symposium on Reconfigurable Communication-centric Systems-on-Chip (ReCoSoC), pp. 1-8, IEEE, 2018.

This paper presents a new PAPI component to access custom hardware PMCs in the ARTICo $^{3}$ architecture. It is also demonstrated that PAPIFY can access new PAPI-based components without requiring any modification.

[Madroñal'18] D. Madroñal, A. Morvan, R. Lazcano, R. Salvador, K. Desnos, E. Juárez, C. Sanz, "Automatic instrumentation of dataflow applications using papi", in Proceedings of the 15th ACM International Conference on Computing Frontiers, pp. 232-235, ACM, 2018.

This paper introduces a preliminary version of PAPIFY monitoring infrastructure, including its base ideas and a first integration with PREESM.

[Madroñal'17a] D. Madroñal, R. Lazcano, H. Fabelo, S. Ortega, R. Salvador, G. M. Callicó, E. Juárez, C. Sanz, "Energy consumption characterization of a Massively Parallel Processor Array (MPPA) platform running a hyperspectral SVM classifier", in 2017 Conference on Design and Architectures for Signal and Image Processing (DASIP), pp. 1-6, IEEE, 2017.

In this paper, an early work of the energy consumption MPPA model using an SVM algorithm is presented. This work was enhanced in Chapter 5 to provide an application-independent energy consumption MPPA model.

|Lazcano’17b] R. Lazcano, D. Madroñal, H. Fabelo, S. Ortega, R. Salvador, G. M. Callicó, E. Juárez, C. Sanz, "Parallel implementation of an iterative PCA algorithm for hyperspectral images on a manycore platform", in 2017 Conference on Design and Architectures for Signal and Image Processing (DASIP), pp. 1-6, IEEE, 2017.

This paper presents an early work on the implementation of the iterative version of PCA algorithm, the so-called NIPALS, onto the MPPA platform.

[Lazcano’17a] R. Lazcano, D. Madroñal, H. Fabelo, S. Ortega, R. Salvador, G. Callicó, E. Juárez, C. Sanz, "Parallel exploitation of a spatial-spectral classification approach for hyperspectral images on RVC-CAL", in High-Performance Computing in Geoscience and Remote Sensing VII, vol. 10430, p. 104300A, International Society for Optics and Photonics, 2017.

This paper presents the implementation on the supervised classification block of the HSI cancer detection processing chain -composed of PCA. SVM and KNN algorithms- using the dataflow language RVC.CAL, This paper was awarded the Best Student Paper Award of the conference.

[Domingo' 17] R. Domingo, R. Salvador, H. Fabelo, D. Madroñal, S. Ortega, R. Lazcano, E. Juárez, G. Callicó, C. Sanz, "High-level design using Intel FPGA OpenCL: 
A hyperspectral imaging spatial-spectral classifier", in 2017 12th International Symposium on Reconfigurable Communication-centric Systems-on-Chip (ReCoSoC), pp. 1-8, IEEE, 2017.

In this paper, a KNN implementation on an Intel Cyclone V SoC using OpenCL is compared to its counterpart baseline $\mathrm{C}$ implementation.

[Salvador'17] R. Salvador, S. Ortega, D. Madroñal, H. Fabelo, R. Lazcano, G. Marrero, E. Juárez, R. Sarmiento, C. Sanz, "HELICoiD: Interdisciplinary and collaborative project for real-time brain cancer detection", in Proceedings of the Computing Frontiers Conference, pp. 313-318, ACM, 2017.

This paper summarizes HELICoiD project presenting 1) its main objectives, 2) its scientific and technical challenges and 3) the main outcomes expected for the end of the project.

[Madroñal'16b] D. Madroñal, R. Lazcano, H. Fabelo, S. Ortega, G. M. Callicó, E. Juárez, C. Sanz, "Hyperspectral image classification using a parallel implementation of the linear SVM on a Massively Parallel Processor Array (MPPA) platform", in 2016 Conference on Design and Architectures for Signal and Image Processing (DASIP), pp. 154-160, IEEE, 2016.

This paper presents the first implementation of the SVM classification algorithm on the MPPA architecture focusing on the obtained performance improvements.

Lazcano’16b R. Lazcano, I. Sidrach-Cardona, D. Madroñal, K. Desnos, M. Pelcat, E. Juárez, C. Sanz, "Parallelism exploitation of a PCA algorithm for hyperspectral images using RVC-CAL", in High-Performance Computing in Geoscience and Remote Sensing VI, vol. 10007, p. 100070H, International Society for Optics and Photonics, 2016.

This paper provides an implementation of the PCA algorithm using the RVCCAL dataflow-based programming language. It mainly focuses on exploiting the inner parallelism of the algorithm.

Madroñal'16a D. Madroñal, H. Fabelo, R. Lazcano, G. Callicó, E. Juárez, C. Sanz, "Parallel implementation of a hyperspectral image linear SVM classifier using RVC-CAL", in High-Performance Computing in Geoscience and Remote Sensing VI, vol. 10007, p. 1000709, International Society for Optics and Photonics, 2016.

This paper provides an implementation of the SVM algorithm using the RVCCAL dataflow-based programming language. It mainly focuses on exploiting the inner parallelism of the algorithm. 


\section{Other dissemination channels}

In addition to the journal papers and the conference publications, the work proposed in this PhD has been disseminated through posters, demos and tutorials.

[Madroñal'19b] D. Madroñal, T. Fanni, "Run-time performance monitoring of hardware accelerators: POSTER", in Proceedings of the 16th ACM International Conference on Computing Frontiers, pp. 289-291, 2019. Poster

In this poster, the MDC PAPI component, oriented to monitor hardware accelerators, is presented. This component is the first configurable PAPI component that is self-adapted using an XML file.

[Lazcano'16a R. Lazcano, D. Madroñal, K. Desnos, M. Pelcat, R. Guerra, S. López, E. Juarez, C. Sanz, "Parallelism Exploitation of a Dimensionality Reduction Algorithm Applied to Hyperspectral Images", in Conference on Design and Architectures for Signal and Image Processing (DASIP), 2016. Poster

This poster presents the first implementation of the PCA classification algorithm on the MPPA architecture focusing on the performance improvements.

Salvador'16 R. Salvador, H. Fabelo, R. Lazcano, S. Ortega, D. Madroñal, G. M. Callicó, E. Juárez, C. Sanz, "HELICoiD tool demonstrator for real-time brain cancer detection", in 2016 Conference on Design and Architectures for Signal and Image Processing (DASIP), pp. 237-238, IEEE, 2016. Demo

In this demonstrator, the potential of combining $\mathrm{HSI}$ with machine learning to locate the boundaries of human brain tumor tissues in real-time using the MPPA platform is shown. This demo was awarded the DASIP 2016 Best Demo Award.

\subsubsection{Research projects}

This thesis has been elaborated under the scope of 3 different projects, in which the student has been actively involved.

- HELICoiD European project -FP7-ICT-2013.9.2 (FET Open) 618080-, granted by the European Commission, aims at using HSI to distinguish, in real-time, healthy and tumor tissues during human brain cancer surgical procedures. As outcomes of the project, 1) a demonstrator being used in the operating theater has been built and 2) a hyperspectral in-vivo brain image database has also been created. In this project, the student has been deeply involved in the acceleration of the algorithms on the MPPA architecture so as to reach real-time responses in the demonstrator. Finally, as explained in Subsection 3.1.1, part of the algorithm developed during HELICoiD is used as the use-case of this $\mathrm{PhD}$. 
- CERBERO European project -H2020-ICT-2016.1 732105-, granted by the European Commission, targets the development of a model-based methodology and toolchain for design, verification and runtime self-awareness of adaptive CPS. For the last point, CERBERO proposes a self-adaptation loop where the system is continuously monitored, optimized and reconfigured, providing high performance and reactiveness to user or environment changes. The student has been deeply involved in the development of a uniform monitoring infrastructure, the so-called PAPIFY tool, explained in Chapter 4, to transparently monitor heterogeneous architectures. Additionally, the self-adaptation loop proposed in this $\mathrm{PhD}$, Chapter 6 , is inspired by the CERBERO approach.

- Plataforma HW/SW distribuida para el procesamiento inteligente de información sensorial heterogénea en aplicaciones de supervisión de grandes espacios naturales (PLATINO) project -TEC2017-86722-C4-4-R-, granted by the Spanish Government, addresses the feasibility of providing a set of solutions to enhance the smart processing of heterogeneous sensor information over distributed heterogeneous platforms. The scenario in which these solutions are assessed is the application of $\mathrm{HSI}$ to smart farming. In this project, the student is tightly involved with the part that aims at providing energy-efficient solutions in high performance heterogeneous architectures through automatic procedures. In this context, this $\mathrm{PhD}$ focuses on the optimization of the energy consumption KPI and the implementation of an automatic energy-awareness HSI system deployment. These procedures have been characterized in Chapter 7 .

\subsubsection{Collaborations}

A series of collaborations with other research groups and a company have been carried out while developing the work presented in this $\mathrm{PhD}$. These collaborations have been performed either as part of the activities associated to the research projects the student has been involved in or as part of a research stay in a company.

- Collaboration with the Instituto Universitario de Microelectrónica Aplicada (IUMA) research group of the Universidad de Las Palmas de Gran Canaria (ULPGC): as part of the activities associated to HELICoiD European project, the student collaborated with this research group in the optimization of the HSI algorithms that have been implemented on the MPPA architecture. Additionally, the student has participated in the activity related with the integration and setup of the HSI processing chain included in the demonstrator of the project. This collaboration has led to the definition of the use-case employed in this $\mathrm{PhD}$ (Subsection 3.1.1) and to the publication of 10 journal and 6 international conference scientific contributions.

- Collaboration with Università degli Studi di Cagliari (UniCA) and Università degli Studi di Sassari (UniSS): in the context of CERBERO European project, 
a one-week research stay has been done at UniCA. During this week, the configurable PAPI component for MDC technology was developed. This component has been used to test the robustness of PAPIFY monitoring infrastructure, as no modifications were required on PAPIFY to transparently access this new PAPI component in a heterogeneous scenario, where both hardware and software $\mathrm{PE}$ s worked in parallel. This collaboration led to the publication of 1 poster and 1 international conference contribution.

- Collaboration with Institut d'Électronique et de Télécommunications de Rennes (IETR) research group of the INSA in the context of CERBEROEuropean project, a tight collaboration has been carried out during the development of this PhD, since PAPIFY monitoring infrastructure has been integrated with both PREESM and SPiDER tools. Additionally, both tools have been extended so as to support energy-awareness in the systems that are automatically generated. The former collaboration, explained in Chapter 4, led to the publication of 1 journal and 1 international conference contribution.

- Collaboration with Kalray: the student carried out a six-month research stay at Kalray as part of the PhD development. During this stay, the student, as explained in Section 5.3, developed a PAPI component for the MPPA Bostan version to be able to use PAPIFY to characterize applications running on this platform. After this, the energy consumption model explained in Chapter 5 was built as part of the collaboration with the company. Later, the PREESM backend for the MPPA architecture was enhanced with 1) support of code generation targeting the $\mathrm{IO}$ subsystem and 2) support of inter-cluster communication by means of a distributed-only memory allocation within the PREESM memory allocation task. These improvements are transparently used during the evaluation of the results of this $\mathrm{PhD}$, explained in Chapter 7 . Finally, the PAPI component was adapted to the third generation of many-cores developed by Kalray, the so-called Coolidge version. From this collaboration, two publications are envisioned: one international conference linked with the transparent monitoring of MPPAPMC $s$ with the new PAPI component; and one journal containing the energy consumption model of the MPPA.

\subsubsection{Open-access products}

To facilitate the access to the products generated during the development of this work, some open-access repositories and tutorials have been made available.

- Repositories: the resources related to the different topics considered in this manuscript can be found in the following repositories:

- PAPIFY: the monitoring infrastructure developed in Chapter 4 can be found in https://gitlab.citsem.upm.es/papify/papify, Additionally, PAPIFY 
is already embedded in PREESM and SPiDER open-source frameworks (https://github.com/preesm).

- Energy modeling: the benchmarks used to extract the power measurement of the energy model presented in Chapter 5 can be found in https://gitlab.citsem.upm.es/dmadronal/ubenchmarks.

- Energy-awareness: the energy-aware optimization loops have already been embedded within PREESM and SPiDER and are freely accessible through https://github.com/preesm.

- HSI cancer detection application: the dataflow version of the HELICoiD processing chain used to validate the proposed methodology can be found in https://gitlab.citsem.upm.es/dmadronal/helicoidDataflow, This repository contains both PREESM and SPiDER implementations. The hyperspectral images are available at [Fabelo'19b].

- Tutorials: in the context of CERBERO European project, some tutorials have been created in order to learn how to use PAPIFY within PREESM and SPiDER.

- PAPIFY and PREESM a tutorial has been published to help PREESM users to learn how to profile applications. The tutorial is available on PREESM website (https://preesm.github.io/tutos/papify/).

- PAPIFY, PREESM, MDC and ARTICo $^{3}$; in this hands-on tutorial, the toolchain composed of these 4 CERBERO tools is shown. These tools collaborate to ease the design of CPS applications targeting heterogeneous embedded computing infrastructures. The material is available in http://www.cpsschool.eu/tutorial-cerbero/,

- PAPIFy, PREESM, SPiDER, MDCand ARTICo $^{3}$; this hands-on tutorial focuses on how to use these 5 CERBERO tools to implement and manage self-adaptation over an embedded heterogeneous processing platform. The material can be found in the following link: https://www.cerberoh2020.eu/tools-and-tutorials/.

\subsubsection{Grants received}

During the development of this $\mathrm{PhD}$, the student received the following grants:

- Contrato Predoctoral del Programa Propio RR01/2016 grant given by Universidad Politécnica de Madrid (UPM). This grant provided financial support during 3 years and 3 months of the PhD development.

- Ayuda a la Internacionalización de Doctorandos grant given by the Consejo Social of UPM. This grant provided financial support during the first 3 months of the student six-month research stay at Kalray. 
- Ayuda para la realización de una Estancia de Investigación Internacional del Programa Propio grant given by UPM. This grant provided financial support during the second 3 months of the student six-month research stay at Kalray.

\subsubsection{Awards obtained}

Thanks to the development of the work presented in this manuscript, the student obtained the following awards:

- DASIP 2016 Best Demo Award. The associated publication is [Salvador'16] R. Salvador, H. Fabelo, R. Lazcano, S. Ortega, D. Madroñal, G. M. Callicó, E. Juárez, C. Sanz, "HELICoiD tool demonstrator for real-time brain cancer detection", in 2016 Conference on Design and Architectures for Signal and Image Processing (DASIP), pp. 237-238, IEEE, 2016.

- Best Student Paper Award. The associated publication is [Lazcano'17a|R. Lazcano, D. Madroñal, H. Fabelo, S. Ortega, R. Salvador, G. Callicó, E. Juárez, C. Sanz, "Parallel exploitation of a spatial-spectral classification approach for hyperspectral images on RVC-CAL", in High-Performance Computing in Geoscience and Remote Sensing VII, vol. 10430, p. 104300A, International Society for Optics and Photonics, 2017.

- Primer Premio UPM Innovatech 2T Challenge 2018. This award was obtained presenting a technology based on the outcomes of HELICoiDEuropean project.

\subsection{Future research lines}

This $\mathrm{PhD}$ work presented an iterative Y-chart dataflow-based design methodology targeting energy optimization. Additionally, prototypes of this methodology using real toolchains have been built for design time, with PREESM, and runtime, with SPiDER, which have been validated and characterized using an HSI cancer detection application. Nevertheless, some aspects of this work are subject to improvement. Specifically, these future research lines are related to 1) improvements on the HSI cancer detection application -Subsection 8.4.1-, 2) enhancement of the individual blocks composing the optimization loop -Subsection 8.4.2- and 3) thorough characterization of the proposed methodology on the already implemented frameworks and its extensions to others -Subsection 8.4.3-. 


\subsubsection{HSI cancer detection application}

One interesting research line that emerges from this $\mathrm{PhD}$ work is the one related to improving the application of $\mathrm{HSI}$ to delineate tumor tissues in real-time.

Although, as explained in Subsection 7.2.1, a lot of effort has been invested on the implementation of an HSI processing chain on the MPPA, only a part of the HELICoiD algorithm has been accelerated. As a result, as shown in [Fabelo'18b], the accelerated part of the algorithm is now faster than the rest of the algorithm. For this reason, further study on the acceleration of the unsupervised part of the HELICoiD processing chain might result in faster processing and quicker responses to the neurosurgeon, which will enhance the usefulness of the whole system during the surgery.

Additionally, modifications to the HSI processing chain can be studied to improve, for example, the accuracy in the tumor delineation. Some studies have been already done, like [Fabelo'19a], where deep learning techniques like $\overline{C N N}$ and $\overline{D N N}$ are compared to the SVM approach. In this study, promising results are obtained, since the results obtained outperforms the previous ones.

Finally, the dataflow version of the HSI processing chain can be further optimized. Currently, as detailed in Subsection 7.3.3, the performance obtained with this version is around 6 times lower than the one done by hand. In this regard, limitations like the one associated to the covariance computation in the PCA algorithm can be avoided by using, for example, the iterative approach of the PCA algorithm, the so-called NIPALS. This algorithm has already been proved to be useful when the number of bands to compute is low [Lazcano'19a], like in the use-case of this $\mathrm{PhD}$, where only one principal component is required as KNNinput.

\subsubsection{Individual blocks extension}

Since the methodology proposed in this $\mathrm{PhD}$ has been built using 3 independent blocks that have been later combined so as to build the energy-aware optimization loop, further improvements in each individual block can be foreseen.

Concerning the monitoring infrastructure PAPIFY, a dataflow-oriented strategy has been proposed, where different monitoring configurations can be defined for each actor and heterogeneous architectures are supported. However, there are two main characteristics that would be interesting to include in order to fully characterize dataflow applications. On the one hand, monitoring data transmissions among resources would provide information to detect possible bottlenecks in this part of the application execution. The combination of processing and transmission instrumentation would fully characterize the execution of dataflow applications. On the other hand, including a sampling strategy of the different parts of the application would help, for example, in the early detection of faults or bottlenecks. In this regard, feeding a runtime resource manager with periodic information could trigger some reconfiguration protocols if the application gets stuck or one of the processing resources stops working [Palumbo'19b]. 
From the energy modeling point of view, this $\mathrm{PhD}$ has proposed a generic approach to build an application-independent power/energy consumption model of an architecture, exemplified by developing a model for the MPPA-256-N platform. In this line, this methodology should be validated by modeling other architectures. Nevertheless, it should be noted that the methodology has been built to be extended and adapted depending on the target architecture. As a result, it is based on modeling 3 sources independently (resource-active, communication and computation) and uses high-level information and PMC data. Additionally, since the MPPA model has been built with the objective of being able to apply it at runtime, only linear approaches have been considered. However, further studies could be performed in order to use non-linear polynomials to increase the accuracy of the model with the drawback of increasing its complexity.

Finally, regarding the energy-awareness, the proposal is to build an iterative procedure that uses latency-based mapping/scheduling algorithms to obtain systems that are optimized in terms of energy consumption. To do so, the latency-based algorithm is considered as a black-box, whose outputs are characterized in terms of energy consumption and whose inputs are iteratively modified to try a set of different system configurations. As explained in [Pelcat'14b], PREESM implements two different mapping/scheduling algorithms: list and Fast Assignment using Search Technique (FAST). In this work, only the former has been used, as this PhD work aimed at proving that energy savings can be obtained using latency-based algorithms as blackboxes. However, further studies where different latency-based mapping/scheduling algorithms, like FAST, are used can be performed so as to analyze if the benefits in the energy consumption increase/decrease.

\subsubsection{Methodology characterization and extensions}

Finally, the last future research line that is proposed starting from this $\mathrm{PhD}$ work is related to the characterization of the two current implementations of the energy-aware optimization loop carried out during this $\mathrm{PhD}$-explained in Chapter 7 - and its possible extension to other frameworks.

Specifically, each loop implementation has been built to optimize the system in terms of energy consumption in a different stage of the system design. The former focuses on enhancing DSE at design time, included within PREESM design framework. The latter, in turn, aims at performing on-the-fly energy-awareness system optimizations, and has been embedded within SPiDER runtime resource manager. In both cases, an application- and architecture- independent approach has been considered, where only the platform energy consumption model is dependent of the target architecture, as seems logic. However, during this PhD work, only the HSI cancer detection processing chain targeting the MPPA platform has been used to validate and characterize the methodology.

In the case of design time optimization, the target architecture has been always the MPPA platform when using PREESM energy-awareness, as shown in Section 7.3. 
Consequently, it would be interesting to perform an extensive analytical characterization of the methodology when targeting different platforms (PREESM already supports code generation for x86, ARM multi-cores, DSPs and Xilinx Zync SoC) and using different applications, whose workloads vary the proportion between resourceactive and computation energy consumptions. To do so, energy consumption models would be needed for each target platform and a deeply understanding of the energy consumption of every type of architecture would be needed. Furthermore, the same energy-awareness optimization approach proposed in this $\mathrm{PhD}$ can be extended to other dataflow-based design frameworks, since it requires little modifications of latency-based mapping/scheduling algorithms, which is the standard approach taken by this kind of frameworks, e.g., SynDEx.

From the point of view of SPiDER, the runtime optimization loop has been validated using a two-step methodology -Section 7.4-. First, the loop has been used to optimize the HSI processing chain when running on a 16-core x86 architecture, where the energy consumption model has been simulated. After this, the optimization loop has been simulated on the MPPA architecture and the actual energy consumption model has been used to verify that the estimations are accurate enough, with promising results. Consequently, it would be interesting to include the whole optimization loop (monitoring, KPI estimations and energy-aware tuning) within the so-called SPiDER-2, which presents a complete restructuring of the tool and could benefit from this feature. On top of that, if the MPPA architecture is supported by SPiDER-2, the energy consumption model already developed during this $\mathrm{PhD}$ could be directly included within the energy-aware optimization loop. 



\section{Bibliography}

[Ackerman'79]

[Adhianto' 10]

[Agarwal'06]

[Aragón'19]

[Arora' 15]

[Baltussen'19]

[Bogliolo'00]

[Bosilca'13]

[Brandolese'00]
W. B. Ackerman, "Data flow languages", in 1979 International Workshop on Managing Requirements Knowledge (MARK), pp. 1087-1095, IEEE, 1979. 19

L. Adhianto, S. Banerjee, M. Fagan, M. Krentel, G. Marin, J. MellorCrummey, N. R. Tallent, "HPCToolkit: Tools for performance analysis of optimized parallel programs", in Concurrency and Computation: Practice and Experience, vol. 22, no. 6, pp. 685-701, 2010. 24

K. Agarwal, K. Nowka, H. Deogun, D. Sylvester, D. Sylvester, "Power gating with multiple sleep modes", in Proceedings of the 7th International Symposium on Quality Electronic Design, pp. 633-637, IEEE Computer Society, 2006. 32

J. S. Aragón, S. S. Ramírez, R. L. López, D. M. Quintín, R. S. Perea, E. J. Martínez, C. S. Álvaro, "Characterizing Hyperspectral Data Layouts: Performance and Energy Efficiency in Embedded GPUs for PCA-based Dimensionality Reduction", in 2019 XXXIV Conference on Design of Circuits and Integrated Systems (DCIS), pp. 1-6, IEEE, 2019. 14189

M. Arora, S. Manne, I. Paul, N. Jayasena, D. M. Tullsen, "Understanding idle behavior and power gating mechanisms in the context of modern benchmarks on cpu-gpu integrated systems", in 2015 IEEE 21st International Symposium on High Performance Computer Architecture (HPCA), pp. 366-377, IEEE, 2015. 32

E. J. Baltussen, E. N. Kok, S. G. B. de Koning, J. Sanders, A. G. Aalbers, N. F. Kok, G. L. Beets, C. C. Flohil, S. C. Bruin, K. F. Kuhlmann, et al., "Hyperspectral imaging for tissue classification, a way toward smart laparoscopic colorectal surgery", in Journal of biomedical optics, vol. 24, no. 1, p. $016002,2019.8$

A. Bogliolo, L. Benini, G. De Micheli, G. De Micheli, G. De Micheli, "Regression-based RTL power modeling", in ACM Transactions on Design Automation of Electronic Systems (TODAES), vol. 5, no. 3, pp. 337-372, 2000. 27.30

G. Bosilca, A. Bouteiller, A. Danalis, M. Faverge, T. Hérault, J. J. Dongarra, "Parsec: Exploiting heterogeneity to enhance scalability", in Computing in Science \& Engineering, vol. 15, no. 6, pp. 36-45, 2013. 25

C. Brandolese, "A codesign approach to software power estimation for embedded systems", in PhD Dissertation, Politecnico di Milano, Institute of Electronics and Information, 2000. 2627 
[Brucker'07]

[Butenhof'97]

[Carroll'10]

[Casale-Brunet'13]

[Casale-Brunet'15]

[Ceng'08]

[Chacko'03]

[Chandra'01]

[Chen'06]

[Chowdhury'18]

[Cordes'10]

[Cordes'11]
P. Brucker, P. Brucker, Scheduling algorithms, vol. 3, Springer, 2007.

D. R. Butenhof, Programming with POSIX threads, Addison-Wesley Professional, 1997. 16

A. Carroll, G. Heiser, et al., "An Analysis of Power Consumption in a Smartphone.", in USENIX annual technical conference, vol. 14, pp. 21-21, Boston, MA, 2010. 33

S. Casale-Brunet, M. Mattavelli, J. Janneck, "TURNUS: A design exploration framework for dataflow system design", in 2013 IEEE International Symposium on Circuits and Systems (ISCAS2013), pp. 654-654, May 2013, ISSN 0271-4302, doi:10.1109/ISCAS.2013.6571927, 19 25

S. Casale-Brunet, "Analysis and optimization of dynamic dataflow programs", in , 2015. 25

J. Ceng, J. Castrillón, W. Sheng, H. Scharwächter, R. Leupers, G. Ascheid, H. Meyr, T. Isshiki, H. Kunieda, "MAPS: an integrated framework for MPSoC application parallelization", in Proceedings of the 45th annual Design Automation Conference, pp. 754-759, ACM, 2008. 18

A. Chacko, N. Kumar, G. Chacko, R. Athyal, V. Rajshekhar, "Intraoperative ultrasound in determining the extent of resection of parenchymal brain tumours-a comparative study with computed tomography and histopathology", in Acta neurochirurgica, vol. 145, no. 9, pp. 743-748, 2003. 10

R. Chandra, L. Dagum, D. Kohr, R. Menon, D. Maydan, J. McDonald, Parallel programming in OpenMP, Morgan kaufmann, 2001. 117

W. Chen, B. Poirier, "Parallel implementation of efficient preconditioned linear solver for grid-based applications in chemical physics. I: Block Jacobi diagonalization", in Journal of Computational Physics, vol. 219, no. 1, pp. 185-197, 2006. 37

T. Chowdhury, "The role of intraoperative MRI in awake neurosurgical procedures: A systematic review", in Frontiers in oncology, vol. 8, p. 434, 2018. 10

D. Cordes, P. Marwedel, A. Mallik, "Automatic parallelization of embedded software using hierarchical task graphs and integer linear programming", in Proceedings of the eighth IEEE/ACM/IFIP international conference on Hardware/software codesign and system synthesis, pp. 267276, ACM, 2010. 18

D. Cordes, A. Heinig, P. Marwedel, A. Mallik, "Automatic extraction of pipeline parallelism for embedded software using linear programming", in 2011 IEEE 17th International Conference on Parallel and Distributed Systems, pp. 699-706, IEEE, 2011. 18 
[Cordes'12]

[Curtsinger'19]

[Dagum'98]

[Danalis'17]

[Dinechin'13]

[Domingo'17]

[Donald'06]

[Dongarra'01]

[Donno'03]

[Esteva'17]

[Fabelo'16a]
D. Cordes, P. Marwedel, "Multi-objective aware extraction of task-level parallelism using genetic algorithms", in Proceedings of the Conference on Design, Automation and Test in Europe, pp. 394-399, EDA Consortium, 2012. 18

C. Curtsinger, "Parallelism in Practice", in , 2019. 16

L. Dagum, R. Menon, "OpenMP: An industry-standard API for sharedmemory programming”, in Computing in Science \& Engineering, , no. 1, pp. 46-55, 1998. 16

A. Danalis, H. Jagode, S. Ragate, J. Dongarra, et al., "Counter Inspection Toolkit: Making Sense Out of Hardware Performance Events", in International Workshop on Parallel Tools for High Performance Computing, pp. 17-37, Springer, 2017. 24

B. D. de Dinechin, R. Ayrignac, P.-E. Beaucamps, P. Couvert, B. Ganne, P. G. de Massas, F. Jacquet, S. Jones, N. M. Chaisemartin, F. Riss, et al., "A clustered manycore processor architecture for embedded and accelerated applications", in 2013 IEEE High Performance Extreme Computing Conference (HPEC), pp. 1-6, IEEE, 2013. 14

R. Domingo, R. Salvador, H. Fabelo, D. Madroñal, S. Ortega, R. Lazcano, E. Juárez, G. Callicó, C. Sanz, "High-level design using Intel FPGA OpenCL: A hyperspectral imaging spatial-spectral classifier", in 2017 12th International Symposium on Reconfigurable Communicationcentric Systems-on-Chip (ReCoSoC), pp. 1-8, IEEE, 2017. 190

J. Donald, M. Martonosi, "Techniques for multicore thermal management: Classification and new exploration", in ACM SIGARCH Computer Architecture News, vol. 34, pp. 78-88, IEEE Computer Society, 2006. 33

J. Dongarra, K. London, S. Moore, P. Mucci, D. Terpstra, "Using PAPI for hardware performance monitoring on Linux systems", in Conference on Linux Clusters: The HPC Revolution, vol. 5, Linux Clusters Institute, 2001. 54

M. Donno, A. Ivaldi, L. Benini, E. Macii, "Clock-tree power optimization based on RTL clock-gating", in Proceedings of the 40th annual Design Automation Conference, pp. 622-627, ACM, 2003. 32

A. Esteva, B. Kuprel, R. A. Novoa, J. Ko, S. M. Swetter, H. M. Blau, S. Thrun, "Dermatologist-level classification of skin cancer with deep neural networks", in Nature, vol. 542, no. 7639, p. 115, 2017. 10

H. Fabelo, S. Ortega, R. Guerra, G. M. Callicó, A. Szolna, J. F. Piñeiro, M. Tejedor, S. López, R. Sarmiento, "A Novel Use of Hyperspectral Images for Human Brain Cancer Detection using in-Vivo Samples.", in BIOSIGNALS, pp. 311-320, 2016. 11 
[Fabelo'16b]

[Fabelo'18a]

[Fabelo'18b]

[Fabelo'19a]

[Fabelo'19b]

[Fanni’19]

[Fei'17]

[Floc'h'13]

[Florimbi'18]
H. Fabelo, S. Ortega, S. Kabwama, G. M. Callico, D. Bulters, A. Szolna, J. F. Pineiro, R. Sarmiento, "HELICoiD project: A new use of hyperspectral imaging for brain cancer detection in real-time during neurosurgical operations", in Hyperspectral Imaging Sensors: Innovative Applications and Sensor Standards 2016, vol. 9860, p. 986002, International Society for Optics and Photonics, 2016. 11

H. Fabelo, S. Ortega, R. Lazcano, D. Madroñal, G. M Callicó, E. Juárez, R. Salvador, D. Bulters, H. Bulstrode, A. Szolna, et al., "An intraoperative visualization system using hyperspectral imaging to aid in brain tumor delineation", in Sensors, vol. 18, no. 2, p. 430, 2018. 134 188

H. Fabelo, S. Ortega, D. Ravi, B. R. Kiran, C. Sosa, D. Bulters, G. M. Callicó, H. Bulstrode, A. Szolna, J. F. Piñeiro, et al., "Spatio-spectral classification of hyperspectral images for brain cancer detection during surgical operations", in PloS one, vol. 13, no. 3, p. e0193721, 2018. $12] 36[123[188[197$

H. Fabelo, M. Halicek, S. Ortega, M. Shahedi, A. Szolna, J. F. Piñeiro, C. Sosa, A. J. O'Shanahan, S. Bisshopp, C. Espino, et al., "Deep learningbased framework for in vivo identification of glioblastoma tumor using hyperspectral images of human brain", in Sensors, vol. 19, no. 4, p. 920, 2019. 197

H. Fabelo, S. Ortega, A. Szolna, D. Bulters, J. F. Piñeiro, S. Kabwama, A. JO'Shanahan, H. Bulstrode, S. Bisshopp, B. R. Kiran, et al., "InVivo Hyperspectral Human Brain Image Database for Brain Cancer Detection", in IEEE Access, vol. 7, pp. 39 098-39 116, 2019. 11]187]195

T. Fanni, D. Madronal, C. Rubattu, C. Sau, F. Palumbo, E. Juarez, M. Pelcat, C. Sanz, L. Raffo, "Run-time Performance Monitoring of Heterogenous Hw/Sw Platforms Using PAPI", in FSP Workshop 2019; Sixth International Workshop on FPGAs for Software Programmers, pp. 1-10, VDE, 2019. $57[189$

B. Fei, G. Lu, X. Wang, H. Zhang, J. V. Little, M. R. Patel, C. C. Griffith, M. W. El-Diery, A. Y. Chen, "Label-free reflectance hyperspectral imaging for tumor margin assessment: a pilot study on surgical specimens of cancer patients", in Journal of biomedical optics, vol. 22, no. 8, p. 086 009, 2017. 9

A. Floc'h, T. Yuki, A. El-Moussawi, A. Morvan, K. Martin, M. Naullet, M. Alle, L. L'Hours, N. Simon, S. Derrien, et al., "GeCoS: A framework for prototyping custom hardware design flows", in 2013 IEEE 13th International Working Conference on Source Code Analysis and Manipulation (SCAM), pp. 100-105, IEEE, 2013. 18

G. Florimbi, H. Fabelo, E. Torti, R. Lazcano, D. Madroñal, S. Ortega, R. Salvador, F. Leporati, G. Danese, A. Báez-Quevedo, et al., "Accelerating the K-nearest neighbors filtering algorithm to optimize the real-time 
[Ge'10]

[Georgakarakos'17]

[Gerard'17]

[Glantz'90]

[Goulas'13]

[Grandpierre’99]

[Haidar'17]

[Halicek'19a]

[Halicek'19b]

[Han'16]

[Hascoet'18] classification of human brain tumor in hyperspectral images", in Sensors, vol. 18 , no. 7, p. 2314, 2018.

Y. Ge, P. Malani, Q. Qiu, "Distributed task migration for thermal management in many-core systems", in Proceedings of the 47th Design Automation Conference, pp. 579-584, ACM, 2010.

G. Georgakarakos, S. Kanur, J. Lilius, K. Desnos, “Task-based execution of synchronous dataflow graphs for scalable multicore computing", in 2017 IEEE International Workshop on Signal Processing Systems (SiPS), pp. 16, IEEE, 2017. 45

I. J. Gerard, M. Kersten-Oertel, K. Petrecca, D. Sirhan, J. A. Hall, D. L. Collins, "Brain shift in neuronavigation of brain tumors: A review", in Medical image analysis, vol. 35, pp. 403-420, 2017.10

S. A. Glantz, B. K. Slinker, T. B. Neilands, Primer of applied regression and analysis of variance, vol. 309, McGraw-Hill New York, 1990. 3093

G. Goulas, C. Gogos, C. Valouxis, P. Alefragis, N. S. Voros, "Coarse Grained Parallelism Optimization for Multicore Architectures: The ALMA Project Approach.", in ARC, pp. 235-236, Springer, 2013. 18

T. Grandpierre, C. Lavarenne, Y. Sorel, "Optimized rapid prototyping for real-time embedded heterogeneous multiprocessors", in Proceedings of the Seventh International Workshop on Hardware/Software Codesign (CODES'99)(IEEE Cat. No. 99TH8450), pp. 74-78, IEEE, 1999. 19

A. Haidar, H. Jagode, A. YarKhan, P. Vaccaro, S. Tomov, J. Dongarra, "Power-aware computing: Measurement, control, and performance analysis for Intel Xeon Phi", in High Performance Extreme Computing Conference (HPEC), 2017 IEEE, pp. 1-7, IEEE, 2017. 23

M. Halicek, H. Fabelo, S. Ortega, G. M. Callico, B. Fei, "In-vivo and ex-vivo tissue analysis through hyperspectral imaging techniques: Revealing the invisible features of cancer", in Cancers, vol. 11, no. 6, p. 756, 2019.78 8910

M. Halicek, J. V. Little, X. Wang, A. Y. Chen, B. Fei, "Optical biopsy of head and neck cancer using hyperspectral imaging and convolutional neural networks", in Journal of biomedical optics, vol. 24, no. 3, p. 036 007, 2019. 9]

Z. Han, A. Zhang, X. Wang, Z. Sun, M. D. Wang, T. Xie, "In vivo use of hyperspectral imaging to develop a noncontact endoscopic diagnosis support system for malignant colorectal tumors", in Journal of biomedical optics, vol. 21, no. 1, p. 016001,2016 . [8

J. Hascoet, Contributions to Software Runtime for Clustered Manycores Applied to Embedded and High-Performance Applications, Ph.D. thesis, 2018. 14 [15] 39$] 142$ 
[Heulot'14] J. Heulot, M. Pelcat, K. Desnos, J.-F. Nezan, S. Aridhi, "Spider: A synchronous parameterized and interfaced dataflow-based rtos for multicore dsps", in Education and Research Conference (EDERC), 2014 6th European Embedded Design in, pp. 167-171, IEEE, 2014. 20

[Holmbacka'13] S. Holmbacka, W. Lund, S. Lafond, J. Lilius, “Task migration for dynamic power and performance characteristics on many-core distributed operating systems", in 2013 21st Euromicro International Conference on Parallel, Distributed, and Network-Based Processing, pp. 310-317, IEEE, 2013. 3334

[Hoque'17] R. Hoque, T. Herault, G. Bosilca, J. Dongarra, "Dynamic task discovery in parsec: A data-flow task-based runtime", in Proceedings of the 8th Workshop on Latest Advances in Scalable Algorithms for Large-Scale Systems, p. 6, ACM, 2017. 25

[Huang'95] C. X. Huang, B. Zhang, A.-C. Deng, B. Swirski, "The design and implementation of PowerMill", in In Proceedings ofthe International Symposium on Low Power Design, Citeseer, 1995. 2728

[Isshiki'08] T. Isshiki, D. Li, H. Kunieda, "Multiprocessor SoC design framework on Tightly-Coupled Thread model", in 2008 International SoC Design Conference, vol. 1, pp. I-56, IEEE, 2008. 18

[Jacobs'08] L. Jacobs, "Positive margins: the challenge continues for breast surgeons", in Annals of surgical oncology, vol. 15, no. 5, pp. 1271-1272, 2008. 8

[Jayanthi'11] J. Jayanthi, G. Nisha, S. Manju, E. Philip, P. Jeemon, K. Baiju, V. Beena, N. Subhash, "Diffuse reflectance spectroscopy: diagnostic accuracy of a non-invasive screening technique for early detection of malignant changes in the oral cavity", in BMJ open, vol. 1, no. 1, p. e000 071, 2011.9

[Jejurikar'04] R. Jejurikar, C. Pereira, R. Gupta, "Leakage aware dynamic voltage scaling for real-time embedded systems", in Proceedings of the 41st annual Design Automation Conference, pp. 275-280, ACM, 2004. 25]26] 31

[Jiang'05]

H. Jiang, M. Marek-Sadowska, S. R. Nassif, "Benefits and costs of powergating technique", in 2005 International Conference on Computer Design, pp. 559-566, IEEE, 2005. 32

[Johansen'20] T. H. Johansen, K. Møllersen, S. Ortega, H. Fabelo, A. Garcia, G. M. Callico, F. Godtliebsen, "Recent advances in hyperspectral imaging for melanoma detection", in Wiley Interdisciplinary Reviews: Computational Statistics, vol. 12, no. 1, p. e1465, 2020. ${ }^{9}$

[Kamble'97] M. B. Kamble, K. Ghose, "Analytical energy dissipation models for low power caches", in Proceedings of 1997 International symposium on low power electronics and design, pp. 143-148, IEEE, 1997. 30 
[Kathuria'11]

[Kazianka'08]

[Kienhuis'01]

[Kleinbaum'88]

[Knüpfer’08]

[Lazcano'16a]

[Lazcano'16b]

[Lazcano'17a]

[Lazcano'17b]

[Lazcano'17c]
J. Kathuria, M. Ayoubkhan, A. Noor, "A review of clock gating techniques", in MIT International Journal of Electronics and Communication Engineering, vol. 1, no. 2, pp. 106-114, 2011. 33

H. Kazianka, R. Leitner, J. Pilz, "Segmentation and classification of hyperspectral skin data", in Data Analysis, Machine Learning and Applications, pp. 245-252, Springer, 2008. 10

B. Kienhuis, E. F. Deprettere, P. Van der Wolf, K. Vissers, "A methodology to design programmable embedded systems", in International Workshop on Embedded Computer Systems, pp. 18-37, Springer, 2001. 2

D. G. Kleinbaum, L. L. Kupper, K. E. Muller, A. Nizam, Applied regression analysis and other multivariable methods, vol. 601, Duxbury Press Belmont, CA, 1988. $\frac{30}{20}$

A. Knüpfer, H. Brunst, J. Doleschal, M. Jurenz, M. Lieber, H. Mickler, M. S. Müller, W. E. Nagel, “The vampir performance analysis tool-set”, in Tools for High Performance Computing, pp. 139-155, 2008. 24

R. Lazcano, D. Madroñal, K. Desnos, M. Pelcat, R. Guerra, S. López, E. Juarez, C. Sanz, "Parallelism Exploitation of a Dimensionality Reduction Algorithm Applied to Hyperspectral Images", in Conference on Design and Architectures for Signal and Image Processing (DASIP), 2016. 192

R. Lazcano, I. Sidrach-Cardona, D. Madroñal, K. Desnos, M. Pelcat, E. Juárez, C. Sanz, "Parallelism exploitation of a PCA algorithm for hyperspectral images using RVC-CAL", in High-Performance Computing in Geoscience and Remote Sensing VI, vol. 10007, p. 100070H, International Society for Optics and Photonics, 2016. 191

R. Lazcano, D. Madroñal, H. Fabelo, S. Ortega, R. Salvador, G. Callicó, E. Juárez, C. Sanz, "Parallel exploitation of a spatial-spectral classification approach for hyperspectral images on RVC-CAL", in High-Performance Computing in Geoscience and Remote Sensing VII, vol. 10430, p. 104300A, International Society for Optics and Photonics, 2017. 190]196

R. Lazcano, D. Madroñal, H. Fabelo, S. Ortega, R. Salvador, G. M. Callicó, E. Juárez, C. Sanz, "Parallel implementation of an iterative PCA algorithm for hyperspectral images on a manycore platform", in 2017 Conference on Design and Architectures for Signal and Image Processing (DASIP), pp. 16, IEEE, 2017. 190

R. Lazcano, D. Madroñal, R. Salvador, K. Desnos, M. Pelcat, R. Guerra, H. Fabelo, S. Ortega, S. López, G. M. Callicó, et al., "Porting a PCAbased hyperspectral image dimensionality reduction algorithm for brain cancer detection on a manycore architecture", in Journal of Systems Architecture, vol. 77, pp. 101-111, 2017. $37[130] 188$ 
[Lazcano'19a] R. Lazcano, D. Madroñal, H. Fabelo, S. Ortega, R. Salvador, G. Callico, E. Juarez, C. Sanz, "Adaptation of an iterative PCA to a manycore architecture for hyperspectral image processing", in Journal of Signal Processing Systems, vol. 91, no. 7, pp. 759-771, 2019. 187197

[Lazcano'19b] R. Lazcano, D. Madroñal, G. Florimbi, J. Sancho, S. Sanchez, R. Leon, H. Fabelo, S. Ortega, E. Torti, R. Salvador, et al., "Parallel Implementations Assessment of a Spatial-Spectral Classifier for Hyperspectral Clinical Applications", in IEEE Access, vol. 7, pp. 152316-152 333, 2019. $13[14[28[132[186$

[Lazcano'20] R. Lazcano, D. Madroñal, E. Juarez, P. Clauss, "Runtime multi-versioning and specialization inside a memoized speculative loop optimizer", in Proceedings of the 29th International Conference on Compiler Construction, pp. 96-107, 2020. 189

[Le Sueur'10] E. Le Sueur, G. Heiser, "Dynamic voltage and frequency scaling: The laws of diminishing returns", in Proceedings of the 2010 international conference on Power aware computing and systems, pp. 1-8, 2010. 33

[Lee'87]

E. A. Lee, D. G. Messerschmitt, "Synchronous data flow", in Proceedings of the IEEE, vol. 75, no. 9, pp. 1235-1245, Sept 1987, ISSN 0018-9219, doi: 10.1109/PROC.1987.13876, 20

[Li'98]

[Lim'14]

[Lorencs'16]

Y. Li, Y. Li, J. Henkel, "A framework for estimation and minimizing energy dissipation of embedded HW/SW systems", in Proceedings of the 35th annual Design Automation Conference, pp. 188-193, ACM, 1998. 22

R. V. Lim, D. Carrillo-Cisneros, W. Alkowaileet, I. Scherson, "Computationally efficient multiplexing of events on hardware counters", in Linux Symposium, pp. 101-110, Citeseer, 2014.

A. Lorencs, J. Sinica-Sinavskis, D. Jakovels, I. Mednieks, "MelanomaNevus Discrimination Based on Image Statistics in Few Spectral Channels", in Elektronika ir Elektrotechnika, vol. 22, no. 2, pp. 66-72, 2016. 10

[Lu'17]

G. Lu, J. V. Little, X. Wang, H. Zhang, M. R. Patel, C. C. Griffith, M. W. ElDeiry, A. Y. Chen, B. Fei, "Detection of head and neck cancer in surgical specimens using quantitative hyperspectral imaging", in Clinical Cancer Research, vol. 23, no. 18, pp. 5426-5436, 2017. 9

[Macken'90] P. Macken, M. Degrauwe, M. Van Paemel, H. Oguey, “A voltage reduction technique for digital systems", in 1990 37th IEEE International Conference on Solid-State Circuits, pp. 238-239, IEEE, 1990. 33

[Madroñal'16a] D. Madroñal, H. Fabelo, R. Lazcano, G. Callicó, E. Juárez, C. Sanz, "Parallel implementation of a hyperspectral image linear SVM classifier using RVC-CAL", in High-Performance Computing in Geoscience and Remote Sensing VI, vol. 10007, p. 1000709, International Society for Optics and Photonics, 2016. 191 
[Madroñal'16b] D. Madroñal, R. Lazcano, H. Fabelo, S. Ortega, G. M. Callicó, E. Juárez, C. Sanz, "Hyperspectral image classification using a parallel implementation of the linear SVM on a Massively Parallel Processor Array (MPPA) platform", in 2016 Conference on Design and Architectures for Signal and Image Processing (DASIP), pp. 154-160, IEEE, 2016. 191

[Madroñal'17a] D. Madroñal, R. Lazcano, H. Fabelo, S. Ortega, R. Salvador, G. M. Callicó, E. Juárez, C. Sanz, "Energy consumption characterization of a Massively Parallel Processor Array (MPPA) platform running a hyperspectral SVM classifier", in 2017 Conference on Design and Architectures for Signal and Image Processing (DASIP), pp. 1-6, IEEE, 2017. 190

[Madroñal'17b] D. Madroñal, R. Lazcano, R. Salvador, H. Fabelo, S. Ortega, G. M. Callicó, E. Juarez, C. Sanz, "SVM-based real-time hyperspectral image classifier on a manycore architecture", in Journal of Systems Architecture, vol. 80, pp. 30-40, 2017. $37[133[148] 188$

[Madroñal'18] D. Madroñal, A. Morvan, R. Lazcano, R. Salvador, K. Desnos, E. Juárez, C. Sanz, "Automatic instrumentation of dataflow applications using papi", in Proceedings of the 15th ACM International Conference on Computing Frontiers, pp. 232-235, ACM, 2018. $58[190$

[Madronal'19a] D. Madronal, F. Arrestier, J. Sancho, A. Morvan, R. Lazcano, K. Desnos, R. Salvador, D. Menard, E. Juarez, C. Sanz, "PAPIFY: Automatic Instrumentation and Monitoring of Dynamic Dataflow Applications Based on PAPI", in IEEE Access, vol. 7, pp. 111801-111812, 2019. $52[58,60,187$

[Madroñal'19b] D. Madroñal, T. Fanni, "Run-time performance monitoring of hardware accelerators: POSTER", in Proceedings of the 16th ACM International Conference on Computing Frontiers, pp. 289-291, 2019. 192

[Makantasis'15] K. Makantasis, K. Karantzalos, A. Doulamis, N. Doulamis, "Deep supervised learning for hyperspectral data classification through convolutional neural networks", in 2015 IEEE International Geoscience and Remote Sensing Symposium (IGARSS), pp. 4959-4962, IEEE, 2015. 10

[March'13]

J. L. March, J. Sahuquillo, S. Petit, H. Hassan, J. Duato, "Poweraware scheduling with effective task migration for real-time multicore embedded systems", in Concurrency and Computation: Practice and Experience, vol. 25, no. 14, pp. 1987-2001, 2013. 34

[Martel'18]

E. Martel, R. Lazcano, J. López, D. Madroñal, R. Salvador, S. López, E. Juarez, R. Guerra, C. Sanz, R. Sarmiento, "Implementation of the principal component analysis onto high-performance computer facilities for hyperspectral dimensionality reduction: Results and comparisons", in Remote Sensing, vol. 10, no. 6, p. 864, 2018. 187

[Maruyama'17] Y. Maruyama, S. Kato, T. Azumi, "Exploring Scalable Data Allocation and Parallel Computing on NoC-based Embedded Many Cores", in 2017 IEEE 
International Conference on Computer Design (ICCD), pp. 225-228, IEEE, 2017. 15 79

[McCraw'14] H. McCraw, J. Ralph, A. Danalis, J. Dongarra, "Power monitoring with PAPI for extreme scale architectures and dataflow-based programming models", in 2014 IEEE International Conference on Cluster Computing (CLUSTER), pp. 385-391, IEEE, 2014. 25

[Meloni' 19] P. Meloni, D. Loi, P. Busia, G. Deriu, A. D. Pimentel, D. Sapra, T. Stefanov, S. Minakova, F. Conti, L. Benini, et al., "Optimization and deployment of CNNs at the edge: the ALOHA experience", in Proceedings of the 16th ACM International Conference on Computing Frontiers, pp. 326-332, ACM, 2019. 20

[Moore'65] G. E. Moore, et al., "Cramming more components onto integrated circuits", 1965. 12

[Mucci'07] P. J. Mucci, "PapiEx-execute arbitrary application and measure hardware performance counters with PAPI", 2007. 24

[Mughees'17] A. Mughees, A. Ali, L. Tao, "Hyperspectral image classification via shapeadaptive deep learning", in 2017 IEEE International Conference on Image Processing (ICIP), pp. 375-379, IEEE, 2017. 10

[Nachbar'94] F. Nachbar, W. Stolz, T. Merkle, A. B. Cognetta, T. Vogt, M. Landthaler, P. Bilek, O. Braun-Falco, G. Plewig, "The ABCD rule of dermatoscopy: high prospective value in the diagnosis of doubtful melanocytic skin lesions", in Journal of the American Academy of Dermatology, vol. 30, no. 4, pp. 551-559, 1994. 10

[Nogues'15] E. Nogues, R. Berrada, M. Pelcat, D. Menard, E. Raffin, "A DVFS based HEVC decoder for energy-efficient software implementation on embedded processors", in 2015 IEEE International Conference on Multimedia and Expo (ICME), pp. 1-6, IEEE, 2015. 108

[Nogues'16a] E. Nogues, Energy optimization of Signal Processing on MPSoCs and its Application to Video Decoding, Ph.D. thesis, Rennes, INSA, 2016. 26

[Nogues'16b] E. Nogues, M. Pelcat, D. Menard, A. Mercat, "Energy efficient scheduling of real time signal processing applications through combined DVFS and DPM", in 2016 24th Euromicro International Conference on Parallel, Distributed, and Network-Based Processing (PDP), pp. 622-626, IEEE, 2016. 26,30

[Orditura'14] M. Orditura, G. Galizia, V. Sforza, V. Gambardella, A. Fabozzi, M. M. Laterza, F. Andreozzi, J. Ventriglia, B. Savastano, A. Mabilia, et al., "Treatment of gastric cancer", in World journal of gastroenterology: WJG, vol. 20, no. 7 , p. $1635,2014.8$

[Ortega'17] S. Ortega, H. Fabelo, R. Camacho, M. Plaza, G. Callico, R. Lazcano, D. Madroñal, R. Salvador, E. Juárez, R. Sarmiento, "P03. 18 Detection of 


\begin{abstract}
human brain cancer in pathological slides using hyperspectral images", in Neuro-oncology, vol. 19, no. Suppl 3, p. iii37, 2017. 188

[Owens'08]

[Pagano'18]

[Palumbo'19a]

J. D. Owens, M. Houston, D. Luebke, S. Green, J. E. Stone, J. C. Phillips, "GPU computing", in , 2008. 13

B. Pagano, C. Pasteur, G. Siegel, R. Kníźek, "A model based safety critical flow for the AURIX multi-core platform", in Embedded Real-Time Software and Systems (ERTS'18), 2018. 2]

F. Palumbo, T. Fanni, C. Sau, L. Pulina, L. Raffo, M. Masin, E. Shindin, P. S. de Rojas, K. Desnos, M. Pelcat, et al., "CERBERO: Cross-layer modElbased fRamework for multi-oBjective dEsign of reconfigurable systems in unceRtain hybRid envirOnments: Invited paper: CERBERO teams from UniSS, UniCA, IBM Research, TASE, INSA-Rennes, UPM, USI, Abinsula, AmbieSense, TNO, S\&T, CRF", in Proceedings of the 16th ACM International Conference on Computing Frontiers, pp. 320-325, ACM, 2019. 202106
\end{abstract}

[Palumbo'19b]

[Panasyuk'07]

[Pascual'16]

[Pelcat'14a]

[Pelcat'14b]

[Pelcat'16]
F. Palumbo, T. Fanni, C. Sau, A. Rodríguez, D. Madroñal, K. Desnos, A. Morvan, M. Pelcat, C. Rubattu, R. Lazcano, et al., "Hardware/Software Self-adaptation in CPS: The CERBERO Project Approach", in International Conference on Embedded Computer Systems, pp. 416-428, Springer, 2019. 21 $189[197$

S. V. Panasyuk, S. Yang, D. V. Faller, D. Ngo, R. A. Lew, J. E. Freeman, A. E. Rogers, "Medical hyperspectral imaging to facilitate residual tumor identification during surgery", in Cancer biology \& therapy, vol. 6, no. 3, pp. 439-446, 2007. 9

M. Pascual, S. Salvans, M. Pera, "Laparoscopic colorectal surgery: current status and implementation of the latest technological innovations", in World Journal of Gastroenterology, vol. 22, no. 2, p. 704, 2016. ${ }^{8}$

M. Pelcat, K. Desnos, J. Heulot, C. Guy, J.-F. Nezan, S. Aridhi, "Preesm: A dataflow-based rapid prototyping framework for simplifying multicore DSP programming", in Education and Research Conference (EDERC), 2014 6th European Embedded Design in, pp. 36-40, Sept 2014, doi: 10.1109/EDERC.2014.6924354, 220

M. Pelcat, K. Desnos, J. Heulot, C. Guy, J.-F. Nezan, S. Aridhi, "Preesm: A dataflow-based rapid prototyping framework for simplifying multicore dsp programming", in 2014 6th european embedded design in education and research conference (EDERC), pp. 36-40, IEEE, 2014. 198

M. Pelcat, K. Desnos, L. Maggiani, Y. Liu, J. Heulot, J.-F. Nezan, S. S. Bhattacharyya, "Models of architecture: Reproducible efficiency evaluation for signal processing systems", in 2016 IEEE International Workshop on Signal Processing Systems (SiPS), pp. 121-126, IEEE, 2016. 28 
[Pelcat'17]

[Petrecca'13]

[Pimentel'06]

[Pimentel'16]

[Piscitelli'12]

[Potlapally'01]

[Quan Ho'18]

[Regeling'16]

[Ren'13]
M. Pelcat, A. Mercat, K. Desnos, L. Maggiani, Y. Liu, J. Heulot, J.-F. Nezan, W. Hamidouche, D. Ménard, S. S. Bhattacharyya, "Reproducible evaluation of system efficiency with a model of architecture: From theory to practice", in IEEE Transactions on Computer-Aided Design of Integrated Circuits and Systems, vol. 37, no. 10, pp. 2050-2063, 2017. $2 8 \longdiv { 2 9 } [ 3 0$

K. Petrecca, M.-C. Guiot, V. Panet-Raymond, L. Souhami, "Failure pattern following complete resection plus radiotherapy and temozolomide is at the resection margin in patients with glioblastoma", in Journal of neurooncology, vol. 111, no. 1, pp. 19-23, 2013. 10

A. D. Pimentel, C. Erbas, S. Polstra, "A systematic approach to exploring embedded system architectures at multiple abstraction levels", in IEEE Transactions on Computers, vol. 55, no. 2, pp. 99-112, 2006. 20212

A. D. Pimentel, "Exploring exploration: A tutorial introduction to embedded systems design space exploration", in IEEE Design \& Test, vol. 34, no. 1, pp. 77-90, 2016. 19

R. Piscitelli, A. D. Pimentel, "Design space pruning through hybrid analysis in system-level design space exploration", in Proceedings of the Conference on Design, Automation and Test in Europe, pp. 781-786, EDA Consortium, 2012. 108

N. R. Potlapally, A. Raghunathan, G. Lakshminarayana, M. S. Hsiao, S. T. Chakradhar, "Accurate power macro-modeling techniques for complex RTL circuits", in VLSI Design 2001. Fourteenth International Conference on VLSI Design, pp. 235-241, IEEE, 2001.2728

[Pourreza-Shahri'13] R. Pourreza-Shahri, F. Saki, N. Kehtarnavaz, P. Leboulluec, H. Liu, "Classification of ex-vivo breast cancer positive margins measured by hyperspectral imaging", in 2013 IEEE International Conference on Image Processing, pp. 1408-1412, IEEE, 2013. ${ }^{8}$

M. Quan Ho, Optimization of data transfer on many-core processors, applied to dense linear algebra and stencil computations, Ph.D. thesis, Laboratoire d'Informatique de Grenoble, 2018. 100

B. Regeling, W. Laffers, A. O. Gerstner, S. Westermann, N. A. Müller, K. Schmidt, J. Bendix, B. Thies, "Development of an image pre-processor for operational hyperspectral laryngeal cancer detection", in Journal of biophotonics, vol. 9, no. 3, pp. 235-245, 2016. 9

R. Ren, J. Wei, E. Juarez, M. Garrido, C. Sanz, F. Pescador, "A PMCdriven methodology for energy estimation in RVC-CAL video codec specifications", in Signal Processing: Image Communication, vol. 28, no. 10, pp. 1303-1314, 2013. ${ }^{3}$ 
[Ren'14]

[Reyes'13]

[Roux'16]

[Roux'17]

[Salvador'16]

[Salvador'17]

[Schlütter'14]

[Shang'02]

[Sorel'05a]

[Sorel'05b]
R. Ren, E. Juarez, C. Sanz, M. Raulet, F. Pescador, "Energy estimation models for video decoders: reconfigurable video coding-CAL casestudy", in IET Computers \& Digital Techniques, vol. 9, no. 1, pp. 3-15, 2014. 3

I. R. Reyes, I. V. Fedyushkina, V. S. Skvortsov, D. A. Filimonov, "Prediction of progesterone receptor inhibition by high-performance neural network algorithm", in Int. J. Math. Model. Meth. Appl. Sci., vol. 7, no. 3, pp. 303-310, 2013. 13

B. Roux, M. Gautier, O. Sentieys, S. Derrien, "Communication-Based Power Modelling for Heterogeneous Multiprocessor Architectures", in 2016 IEEE 10th International Symposium on Embedded Multicore/Manycore Systems-on-Chip (MCSOC), pp. 209-216, IEEE, 2016. 14,28229

B. Roux, Methodology and Tools for Energy-aware Task Mapping on Heterogeneous Multiprocessor Architectures, Ph.D. thesis, 2017. 66

R. Salvador, H. Fabelo, R. Lazcano, S. Ortega, D. Madroñal, G. M. Callicó, E. Juárez, C. Sanz, "HELICoiD tool demonstrator for real-time brain cancer detection", in 2016 Conference on Design and Architectures for Signal and Image Processing (DASIP), pp. 237-238, IEEE, 2016. 192196

R. Salvador, S. Ortega, D. Madroñal, H. Fabelo, R. Lazcano, G. Marrero, E. Juárez, R. Sarmiento, C. Sanz, "HELICoiD: Interdisciplinary and collaborative project for real-time brain cancer detection", in Proceedings of the Computing Frontiers Conference, pp. 313-318, ACM, 2017. 111]12]

M. Schlütter, B. Mohr, L. Morin, P. Philippen, M. Geimer, "Profiling Hybrid HMPP Applications with Score-P on Heterogeneous Hardware", in International Conference on Parallel Computing, FZJ-2014-01861, Jülich Supercomputing Center, 2014. 24

L. Shang, A. S. Kaviani, K. Bathala, "Dynamic power consumption in Virtex ${ }^{\mathrm{TM}}$-II FPGA family", in Proceedings of the 2002 ACM/SIGDA tenth international symposium on Field-programmable gate arrays, pp. 157164, ACM, 2002. 32

Y. Sorel, "From modeling/simulation with Scilab/Scicos to optimized distributed embedded real-time implementation with SynDEx", in Proceedings of the International Workshop On Scilab and Open Source Software Engineering, SOSSE, vol. 5, Citeseer, 2005. 18

Y. Sorel, "From modeling/simulation with Scilab/Scicos to optimized distributed embedded real-time implementation with SynDEx", in Proceedings of the International Workshop On Scilab and Open Source Software Engineering, SOSSE'05, Wuhan, China, Oct. 2005, http:// www-rocq.inria.fr/syndex/publications/pubs/sosse05/sosse05.pdf. 19 
[Stamnes'17] J. Stamnes, G. Ryzhikov, M. Biryulina, B. Hamre, L. Zhao, K. Stamnes, "Optical detection and monitoring of pigmented skin lesions", in Biomedical optics express, vol. 8, no. 6, pp. 2946-2964, 2017. 10

[Stripf'13]

T. Stripf, O. Oey, T. Bruckschloegl, J. Becker, G. Rauwerda, K. Sunesen, G. Goulas, P. Alefragis, N. S. Voros, S. Derrien, et al., "Compiling Scilab to high performance embedded multicore systems", in Microprocessors and Microsystems, vol. 37, no. 8, pp. 1033-1049, 2013. 18

[Stummer'06]

W. Stummer, U. Pichlmeier, T. Meinel, O. D. Wiestler, F. Zanella, H.J. Reulen, A.-G. S. Group, et al., "Fluorescence-guided surgery with 5aminolevulinic acid for resection of malignant glioma: a randomised controlled multicentre phase III trial", in The lancet oncology, vol. 7, no. 5, pp. 392-401, 2006. 10

[Stummer'11] W. Stummer, J.-C. Tonn, H. M. Mehdorn, U. Nestler, K. Franz, C. Goetz, A. Bink, U. Pichlmeier, "Counterbalancing risks and gains from extended resections in malignant glioma surgery: a supplemental analysis from the randomized 5-aminolevulinic acid glioma resection study", in Journal of neurosurgery, vol. 114, no. 3, pp. 613-623, 2011.10

[Suriano'18]

L. Suriano, D. Madroñal, A. Rodríguez, E. Juárez, C. Sanz, E. de la Torre, "A unified hardware/software monitoring method for reconfigurable computing architectures using PAPI", in 2018 13th International Symposium on Reconfigurable Communication-centric Systems-on-Chip (ReCoSoC), pp. 1-8, IEEE, 2018. 190

[Terpstra'10] D. Terpstra, H. Jagode, H. You, J. Dongarra, “Collecting performance data with PAPI-C”, in Tools for High Performance Computing 2009, pp. 157173, Springer, 2010. 2222]24

[Thompson'06] S. E. Thompson, S. Parthasarathy, "Moore's law: the future of Si microelectronics", in Materials today, vol. 9, no. 6, pp. 20-25, 2006. 12

[Torun'16]

M. U. Torun, O. Yilmaz, A. N. Akansu, "FPGA, GPU, and CPU implementations of Jacobi algorithm for eigenanalysis", in Journal of Parallel and Distributed Computing, vol. 96, pp. 172-180, 2016. 13

[Vigneswaran'14] N. Vigneswaran, M. D. Williams, "Epidemiologic trends in head and neck cancer and aids in diagnosis", in Oral and Maxillofacial Surgery Clinics, vol. 26, no. 2, pp. 123-141, 2014. 9

[Wiltgen'13]

A. Wiltgen, K. A. Escobar, A. I. Reis, R. P. Ribas, "Power consumption analysis in static CMOS gates", in 2013 26th Symposium on Integrated Circuits and Systems Design (SBCCI), pp. 1-6, IEEE, 2013. 31

[Wu'00]

Q. Wu, M. Pedram, X. Wu, "Clock-gating and its application to low power design of sequential circuits", in IEEE Transactions on Circuits and Systems I: Fundamental Theory and Applications, vol. 47, no. 3, pp. 415420, 2000. 32 
[Wu'04]

[Yang'18]

[Yviquel'13a]

[Yviquel'13b]

[Zhang'12]

[Zherdeva'16]
T.-F. Wu, C.-J. Lin, R. C. Weng, "Probability estimates for multi-class classification by pairwise coupling", in Journal of Machine Learning Research, vol. 5, no. Aug, pp. 975-1005, 2004.

Y. Yang, W. Hu, X. Chen, G. Cao, "Energy-Aware CPU Frequency Scaling for Mobile Video Streaming", in IEEE Transactions on Mobile Computing, 2018. 33

H. Yviquel, A. Lorence, K. Jerbi, G. Cocherel, A. Sanchez, M. Raulet, “Orcc: Multimedia Development Made Easy", in Proceedings of the 21st ACM International Conference on Multimedia, MM '13, pp. 863-866, ACM, 2013, ISBN 978-1-4503-2404-5, doi:10.1145/2502081.2502231, 2

H. Yviquel, A. Lorence, K. Jerbi, G. Cocherel, A. Sanchez, M. Raulet, “Orcc: Multimedia development made easy", in Proceedings of the 21st ACM international conference on Multimedia, pp. 863-866, ACM, 2013. 19

L. Zhang, L. Zhang, D. Tao, X. Huang, "Tensor discriminative locality alignment for hyperspectral image spectral-spatial feature extraction", in IEEE Transactions on Geoscience and Remote Sensing, vol. 51, no. 1, pp. 242-256, 2012. 142

L. A. Zherdeva, I. A. Bratchenko, O. O. Myakinin, A. A. Moryatov, S. V. Kozlov, V. P. Zakharov, "In vivo hyperspectral imaging and differentiation of skin cancer", in Optics in Health Care and Biomedical Optics VII, vol. 10024, p. 100244G, International Society for Optics and Photonics, 2016. 10 